\title{
IMPLANTAÇÃO DE UM CONTROLADOR MULTIMODELOS EM UMA COLUNA DEPROPANIZADORA INDUSTRIAL
}

Dissertação apresentada à Escola Politécnica da Universidade de São Paulo para obtenção do título de Mestre em Engenharia. 


\section{IMPLANTAÇÃO DE UM CONTROLADOR MULTIMODELOS EM UMA COLUNA DEPROPANIZADORA INDUSTRIAL}

Dissertação apresentada à Escola Politécnica da Universidade de São Paulo para obtenção do título de Mestre em Engenharia.

Área de Concentração:

Engenharia Química

Orientador:

Prof. Dr Darci Odloak

SÃO PAULO

Outubro/2001 
Porfírio, Carlos Roberto

Implantação de um Controlador Multimodelos em uma Coluna Depropanizadora Industrial.

São Paulo, 2001.

130 p.

Dissertação (Mestrado) - Escola Politécnica da Universidade de São Paulo. Departamento de Engenharia Química.

1. Controle de Processo 2. Controle Preditivo 3. Coluna Depropanizadora 4. Controle Multimodelos

I. Universidade de São Paulo. Escola Politécnica. Departamento de Engenharia Química II. T 
A todos os meus mestres, que ao longo de minha vida me mostraram o valor da educação, e a Deus por me dar forças para conseguir superar todas as dificuldades enfrentadas nesta jornada. 


\section{AGRADECIMENTOS}

Ao Prof. Dr. Darci Odloak pela orientação e dedicação, tornando possível a realização deste trabalho.

À minha esposa e filho, Ronilda e Gabriel, pela compreensão e incentivo durante a realização deste trabalho.

Ao engenheiro Euclides Almeida Neto pela paciência, apoio e incentivo nas intermináveis discussões durante a realização deste trabalho.

À Petrobras, em nome do engenheiro Antônio Maylinch Teruel, por permitir e incentivar a realização desta especialização profissional.

Ao colega Dr. Oscar Rotava pelas sugestões e revisão final do trabalho.

Ao colega Dr. Marco Antônio Rodrigues pela ajuda na preparação das transparências para a apresentação final deste trabalho.

A meu pai Onofre Porfírio e meus irmãos Maurício, Elcio, Beatriz, Rodrigo e Júnior pelo incentivo durante toda minha vida acadêmica e também pela amizade, carinho e convivência.

Aos Operadores da Unidade de Craqueamento Fluido da RPBC pelo auxílio durante os diversos testes realizados na unidade durante a elaboração deste trabalho. 


\section{SUMÁRIO}

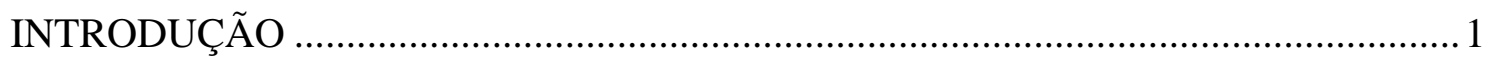

1.1 - Apresentação da problemática de controle de colunas de pureza elevada............ 1

1.2 - Revisão bibliográfica ............................................................................. 2

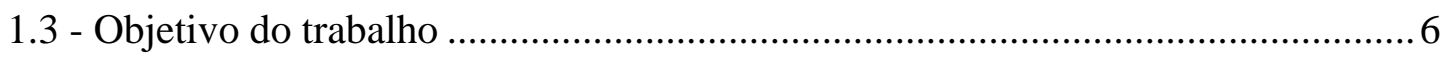

PROBLEMA DE CONTROLE DA COLUNA DEPROPANIZADORA ...................... 8

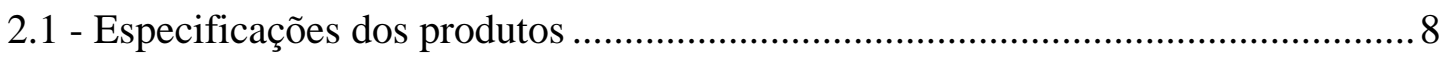

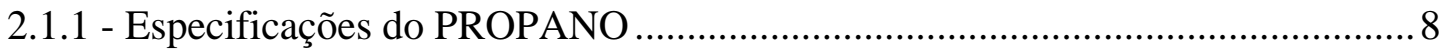

2.1.2 - Especificações do BUTANO ................................................................ 9

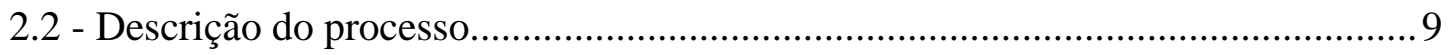

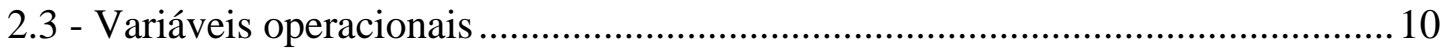

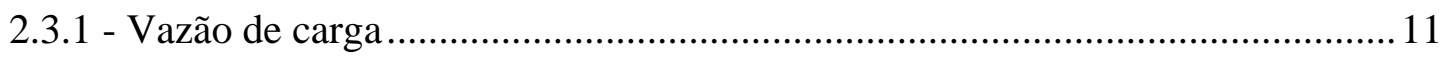

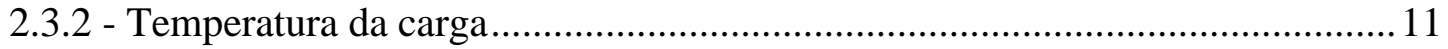

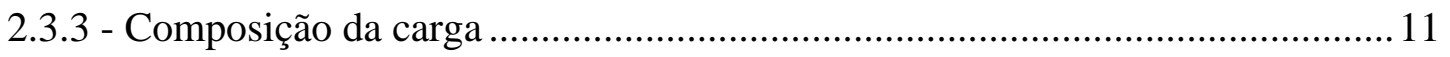

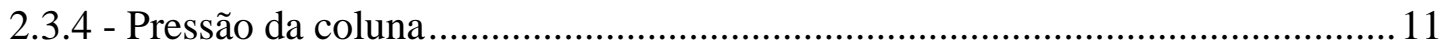

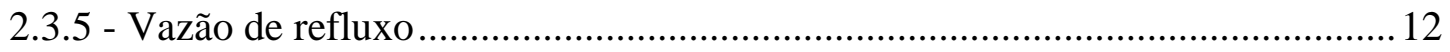

2.3.6 - Vazão do fluido de aquecimento para refervedor .......................................... 12

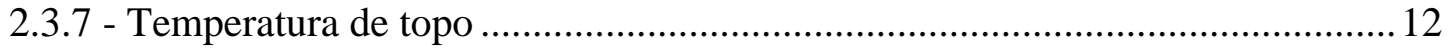

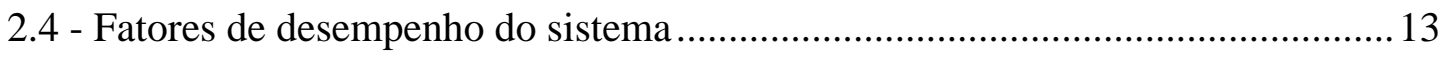

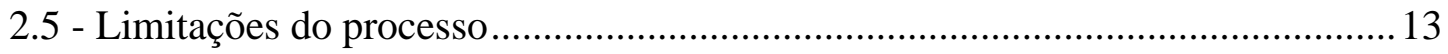

2.6 - Estratégias de controle da coluna depropanizadora ....................................... 13

2.6.1 - Temperatura de retorno do refervedor - vazão de refluxo de topo .................. 14

2.6.2 - Vazão do fluido de aquecimento do refervedor - vazão de refluxo de topo ... 15

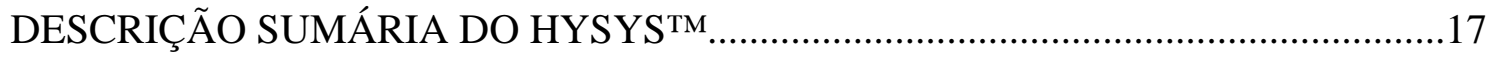

SIMULAÇÃO ESTÁTICA DA DEPROPANIZADORA …........................................ 19

4.1 - Etapas da construção do modelo estático........................................................ 19

4.1.1 - Definição das bases de simulação ................................................................. 19

4.1.2 - Caracterização da carga do sistema......................................................... 21

4.1.3 - Definição das operações unitárias envolvidas no processo............................22 
4.1.4 - Especificação dos graus de liberdade do sistema...........................................24

4.1.5 - Validação do modelo estático …………………………………………......25

4.1.6 - Definição dos estudos da simulação estática.....................................................27

SIMULAÇÃO DINÂMICA DA DEPROPANIZADORA ………………………........29

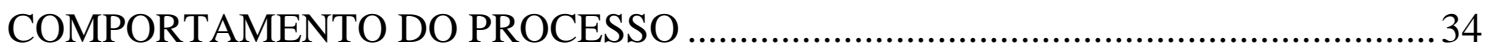

6.1 - Região de Operação e Ganhos do Processo .......................................................... 35

IDENTIFICAÇÃO DOS MODELOS DO SISTEMA ……………...........................4

7.1 - Algoritmo de identificação de modelos na forma analítica...................................4

7.2 - Modelos de Processo Identificados ....................................................................49

ALGORITMOS DE CONTROLE ……………………......................................5

8.1 - MPC com modelo não-paramétrico …………………………………….....5

8.1.1 - Cálculo das predições das variáveis controladas.............................................58

8.1.1.1 - Efeito da última ação de controle .................................................................58

8.1.1.2 - Correção das predições com as leituras da planta ..........................................60

8.1.1.3 - Translação da predição corrigida ................................................................63

8.1.2 - Cálculo das ações de controle .........................................................................64

8.1.2.1 - Trajetória de referência das variáveis controladas .......................................65

8.1.2.2 - Cálculo dos erros das variáveis controladas...................................................67

8.1.2.3 - Algoritmo de controle preditivo...............................................................6

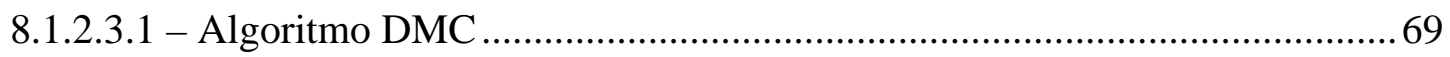

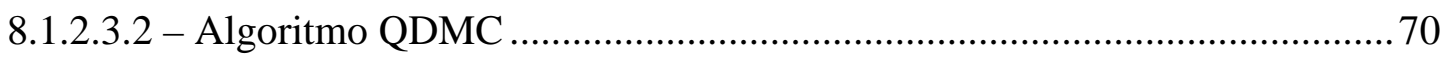

8.1.2.3.2.1 - Restrições de movimentos................................................................... 72

8.1.2.3.2.2 - Restrições de limites ........................................................................ 72

8.2 - MPC com modelo de ordem reduzida............................................................. 74

8.2.1 - Modelo de função de transferência ................................................................. 74

8.2.2 - Predição com modelos paramétricos................................................................ 78

8.2.3 - Cálculo das predições das variáveis controladas............................................... 82

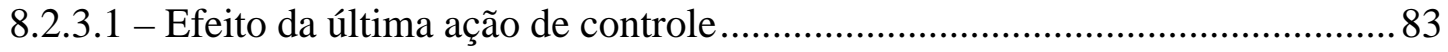

8.2.3.2 - Correção das predições com as leituras da planta .......................................... 84

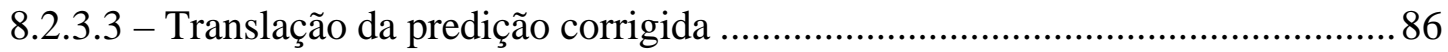

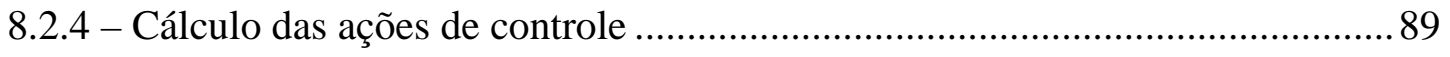

8.2.4.1 - Trajetória de referência das variáveis controladas .........................................89 
8.2.4.2 - Cálculo dos erros das variáveis controladas.............................................90

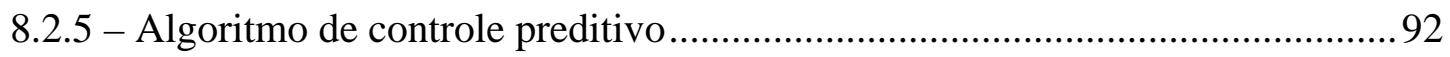

8.2.5.1 - MMPC (Multi-Model Predictive Control) …............................................. 92

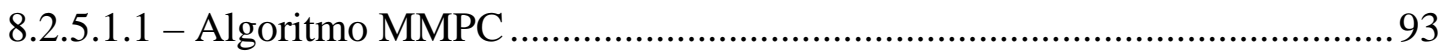

8.2.5.1.2 - Representação de incertezas do sistema..................................................94

APLICAÇÃO DO MPC À COLUNA DEPROPANIZADORA ...................................96

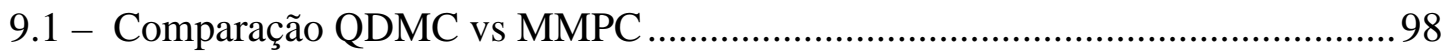

9.1.1 - Ajuste sintonia dos controladores ............................................................99

9.1.2 - QDMC e MMPC com sintonias ajustadas ................................................ 102

9.1 .3 - Alteração no modelo da planta.................................................................... 105

9.2 - Implantação do controlador MMPC na planta industrial ................................ 109

9.2.1 - SICON - Sistema de Controle (da Petrobras)............................................. 118

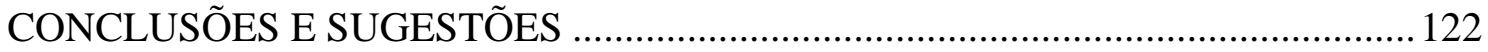

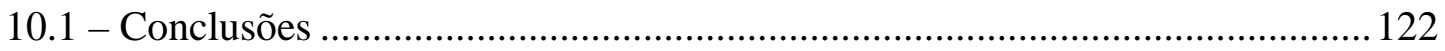

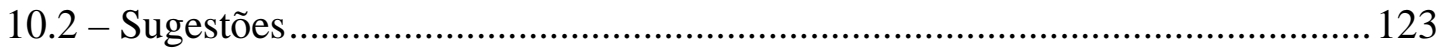

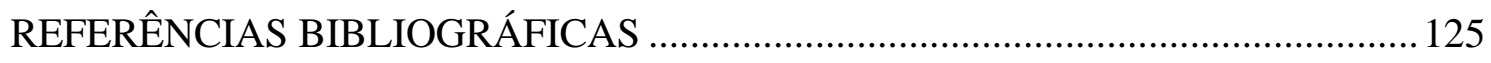

APÊNDICE A - FUNÇÃO DE TRANSFERÊNCIA DOS MODELOS

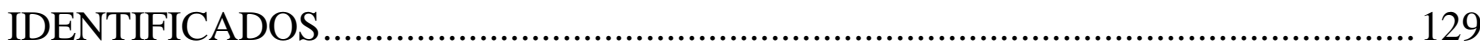




\section{LISTA DE FIGURAS}

Figura 2.1 - Esquema de produção de GLP em refinarias de petróleo ............................ 8

Figura 2.2 - Fluxograma de processo da coluna depropanizadora...............................10

Figura 4.1 - Fluxograma de processo da simulação ...................................................22

Figura 4.2 - Fluxograma de processo coluna simulada .............................................23

Figura 5.1 - Esquema de controle da coluna depropanizadora.................................... 30

Figura 5.2 - Diagrama em blocos da malha fechada. ...............................................33

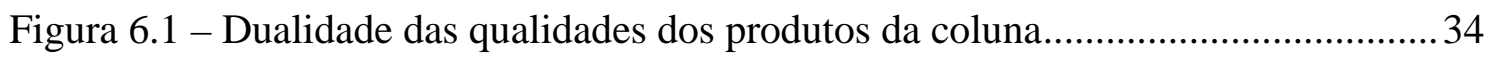

Figura 6.2 - Região de operação das variáveis manipuladas ........................................3 37

Figura 6.3 - Ganhos do C3- versus a vazão de refluxo............................................. 38

Figura 6.4 - Ganhos do C3- versus Vazão de GOP ..................................................... 39

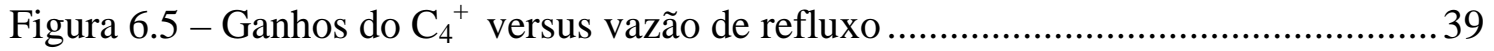

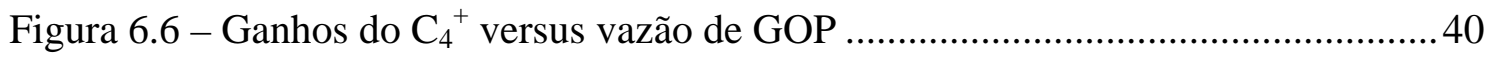

Figura 6.7 - Ganhos da Temperatura de Topo versus vazão de Refluxo........................40

Figura 6.8 - Ganhos da Temperatura de Topo versus vazão de GOP.......................... 41

Figura 7.1 - Região de operação do processo ..................................................... 49

Figura 7.2 -Modelos de resposta ao degrau nas manipuladas. O modelo da planta real é

o FD

Figura 7.3 - Modelos de resposta ao degrau nas perturbações. O modelo da planta real é

o FD.

Figura 9.1.a - Comparação QDMC( - - ) x MMPC( — ) com mesma sintonia ....... 100

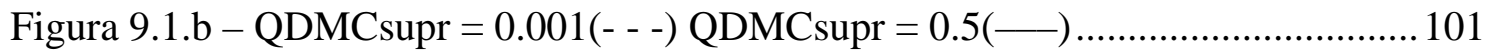

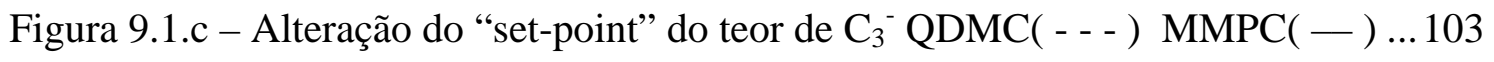

Figura 9.1.d - Alteração da faixa de controle da Ttopo QDMC( - - - ) MMPC( — ).. 104

Figura 9.1.e - Aplicação de degrau na vazão de carga QDMC( - - - ) MMPC ( — ) ... 104

Figura 9.1.f - Aplicação de degrau na TGOP QDMC( - - ) MMPC( - ) ................. 105

Figura 9.1.g - Mudança p/ modelo 1 no instante 20 QDMC( - - ) MMPC( - )....... 106

Figura 9.1.h - Mudança modelo p/ 2 no instante 20 QDMC( - - - ) MMPC( — ) ...... 107

Figura 9.1.i - Mudança modelo para 3 no instante 20 QDMC( - - - ) MMPC( — )..... 107

Figura 9.1.j - Mudança modelo para 4 no instante 20 QDMC( - - ) MMPC( — ).... 108

Figura 9.1.k - Mudança modelo p/ 5 no instante 20 QDMC( - - ) MMPC( — ) ....... 109 
Figura 9.2.a1 - Variáveis controladas em operação típica......................................... 113

Figura 9.2.a2 - Variáveis manipuladas em operação típica ........................................ 113

Figura 9.2.a3 - Variáveis perturbadoras em operação típica .................................... 113

Figura 9.2.b1 - Variáveis controladas teste 1 .................................................... 114

Figura 9.2.b2 - Variáveis manipuladas teste 1..................................................... 114

Figura 9.2.b3 - Variáveis perturbadoras teste 1 ........................................................ 114

Figura 9.2.c1 - Variáveis controladas teste 2 ......................................................... 115

Figura 9.2.c2 - Variáveis manipuladas teste 2 ..................................................... 115

Figura 9.2.c3 - Variáveis perturbadoras teste 2 ................................................... 116

Figura 9.2.d1 - Variáveis controladas teste 3 ....................................................... 117

Figura 9.2.d2 - Variáveis manipuladas teste 3....................................................... 117

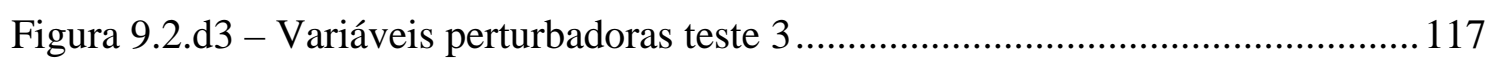

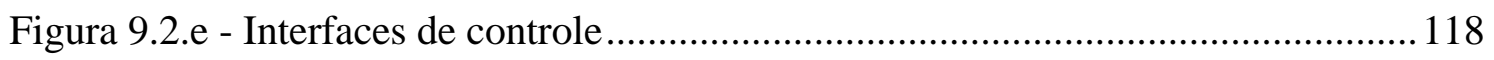

Figura 9.2.f - Tela de configuração/sintonia do controlador .................................... 120

Figura 9.2.g - Tela de configuração/sintonia de variável controlada........................... 120

Figura 9.2.h - Tela de configuração/sintonia de variável manipulada ......................... 121

Figura 9.2.i - Tela de Configuração de Variável Perturbadora .................................... 121 


\section{LISTA DE TABELAS}

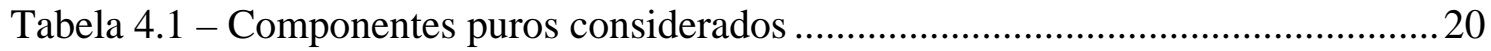

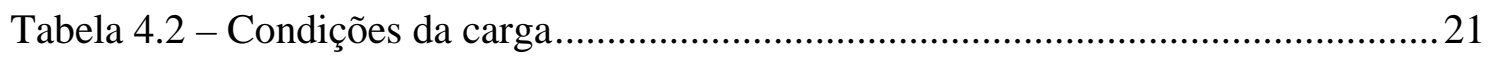

Tabela 4.3 - Composição da carga............................................................................21

Tabela 4.4 - Especificações da simulação ............................................................. 25

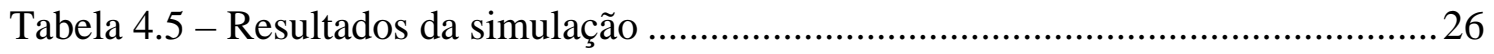

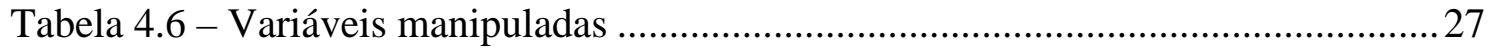

Tabela 4.7 - Restrições do processo ..................................................................27

Tabela 5.1 - Dados geométricos dos equipamentos ...................................................30

Tabela 5.2 - Parâmetros de configuração dos controladores ....................................... 31

Tabela 5.3 - Parâmetros de sintonia dos controladores ........................................... 31

Tabela 6.1 - Parâmetros dos modelos das inferências ..................................................36

Tabela 6.2 - Ganhos do processo em relação à vazão de refluxo ..................................41

Tabela 6.3 - Ganhos do processo para a vazão de GOP........................................... 42

Tabela 6.4 - Ganhos do processo para a vazão de carga ........................................... 42

Tabela 6.5 - Ganhos do processo para a temperatura do fluido de aquecimento ...........42

Tabela 7.1 - Pontos de operação dos modelos do processo........................................50

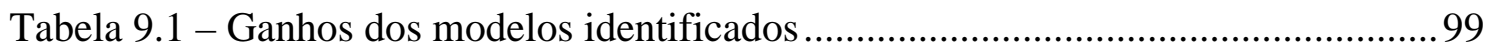

Tabela 9.2: Características dos controladores utilizados ...........................................99

Tabela 9.3 - Parâmetros de sintonia dos controladores para figura 9.1.a .................... 100

Tabela 9.4 - Parâmetros de sintonia dos controladores para figura 9.2.a .................... 101

Tabela 9.5 - Sintonia dos controladores para item 9.1.2 _....................................... 102

Tabela 9.6 - Parâmetros de sintonia do controlador industrial .................................. 112

Tabela A1 - Parâmetros da função de transferência para o modelo 1 .......................... 129

Tabela A2 - Parâmetros da função de transferência para o modelo 2 …...................... 130

Tabela A3 - Parâmetros da função de transferência para o modelo 3 ......................... 130

Tabela A4 - Parâmetros da função de transferência para o modelo 4. ......................... 131

Tabela A5 - Parâmetros da função de transferência para o modelo 5.........................131

Tabela A6 - Parâmetros da função de transferência para modelo da planta industrial 132 


\section{LISTA DE ABREVIATURAS}

\begin{tabular}{|c|c|}
\hline $\mathrm{C}_{3}^{-}$ & $\%$ propano e componentes com pesos moleculares mais baixos \\
\hline $\mathrm{C}_{4}^{+}$ & $\%$ de iso-butano e componentes de maior peso molecular \\
\hline $\mathrm{CV}_{1}$ & Variável controlada 1 \\
\hline $\mathrm{CV}_{2}$ & Variável controlada 2 \\
\hline DDE & Dynamic Data Exchange \\
\hline DEA & Di-Etanol amina \\
\hline $\operatorname{Det}\left(K_{\mathrm{p}}\right)$ & Determinante da matriz de ganhos do processo \\
\hline DMC & Dynamic Matrix Control \\
\hline FCC & Craqueamento catalítico flúido \\
\hline $\mathrm{FC}$ & Controlador de vazão \\
\hline FIR & Finite Impulse Response \\
\hline GLP & Gás Liqüefeito de Petróleo \\
\hline GPC & Generalized Predictive Control \\
\hline HYSYS & Software de simulação de processo da empresa Hyprotech Inc \\
\hline ISE & Integral do erro quadrático \\
\hline LDMC & Linear Dynamic Matrix Control \\
\hline $\mathrm{LC}$ & Controlador de Nível \\
\hline LMI & Linear Matrix Inequality \\
\hline LP & Programação linear \\
\hline $\mathrm{L}_{\mathrm{REB}}$ & Nível de fundo da coluna \\
\hline $\mathrm{L} / \mathrm{V}$ & Relação molar líquido e vapor no estágio da coluna \\
\hline MAC & Model Algorithmic Control \\
\hline Matlab $^{\text {TM }}$ & Software Matemático da empresa Mathworks Inc \\
\hline Mdl & Modelo \\
\hline MMPC & Multi-Model Predictive Control \\
\hline MPC & Controlador preditivo multivariável \\
\hline MPHC & Model Predictive Heuristic Control \\
\hline $\mathrm{MV}_{1}$ & Variável manipulada 1 \\
\hline $\mathrm{MV}_{2}$ & Variável manipulada 2 \\
\hline $\mathrm{NC}$ & Número de componentes do sistema \\
\hline
\end{tabular}




$\begin{array}{ll}\text { OLE } & \text { Object Link Embeding } \\ \text { OP } & \text { Saída do controlador } \\ \text { P }_{\text {COND }} & \text { Pressão no condensador } \\ \text { PI }^{\text {TM }} & \text { Software de supervisão do processo Plant Information } \\ \text { PID } & \text { Controlador proporcional integral e derivativo } \\ \text { PV } & \text { Variável de processo } \\ \text { P\#24 }_{\#} & \text { Pressão no estágio 24 } \\ \text { Qcond } & \text { Calor absorvido na condensação } \\ \text { QDMC } & \text { Quadratic Dynamic Matrix Control } \\ \text { QP } & \text { programação quadrática } \\ \text { QREB } & \text { Carga térmica do refervedor } \\ \text { REFAP } & \text { Refinaria Alberto Pasqualini } \\ \text { REPLAN } & \text { Refinaria de Paulínia } \\ \text { REVAP } & \text { Refinaria do Vale do Paraíba } \\ \text { RPBC } & \text { Refinaria Presidente Bernardes de Cubatão } \\ \text { SDCD } & \text { Sistema Digital de Controle Distribuído } \\ \text { SICON } & \text { Sistema de Controle da Petrobras } \\ \text { SISO } & \text { Sistema monovariável } \\ \text { SP } & \text { Setpoint } \\ \text { SQP } & \text { Programação quadrática sucessiva } \\ \text { TC } & \text { Controlador de temperatura } \\ T_{\text {GOP }} & \text { Temperatura do gasóleo pesado } \\ \text { T }_{\text {TOPO }} & \text { Temperatura de topo da coluna } \\ \text { UA } & \text { Coeficiente global de troca térmica } \\ \text { UFCC } & \text { Unidade de Craqueamento Catalítico Fluido } \\ \text { UGAV } & \text { Unidade de Gasolina de Aviação } \\ \text { USP } & \text { Universidade de São Paulo } \\ V_{\text {BUTANO }} & \text { Vazão de butano } \\ V_{\text {CARGA }} & \quad \text { Vazazão de carga } \\ \text { Vazão de gasóleo pesado de propano } \\ \end{array}$


Vazão de Refluxo de Topo 


\section{LISTA DE SÍMBOLOS}

\begin{tabular}{|c|c|}
\hline$a$ & Termos do denominador do modelo do processo \\
\hline$b$ & Termos do numerador do modelo do processo \\
\hline$c$ & Parâmetro do modelo dependente do tempo \\
\hline$c_{0}$ & Parâmetro do modelo independente do tempo \\
\hline $\mathbf{C}$ & Matriz dos parâmetros do modelo dep. do tempo \\
\hline $\mathbf{C}$ & Matriz diag. dos parâm. do modelo dep. do tempo \\
\hline $\mathbf{C}_{0}$ & Matriz dos parâmetros do modelo indep. do tempo \\
\hline$d$ & Coef. de atualização dos parâmetros do modelo \\
\hline $\mathbf{D}$ & Matriz de coef. de atualização dos parâmetros \\
\hline det & Determinante de uma matriz \\
\hline$E(t)$ & Erro no instante $\mathrm{t}$ \\
\hline$e$ & Erro na controlada antes do controle \\
\hline$\overline{\mathbf{e}}$ & Vetor de erros nas controladas após o controle \\
\hline$e_{k+\infty}$ & Erros das controladas no estado estacionário \\
\hline$F$ & Vazão do fluido \\
\hline FD & Modelo da planta industrial \\
\hline$f(t)$ & Função exponencial do modelo do processo \\
\hline$g(s)$ & Função de transferência contínua do processo \\
\hline $\mathbf{I}_{n y}$ & Matriz diagonal de dimensão $n y$ \\
\hline$J_{k}$ & Função objetivo do controlador preditivo \\
\hline$K$ & Matriz de correção da predição da saída \\
\hline$K_{\mathrm{c}}$ & Ganho do controlador PID \\
\hline $\mathbf{K}_{\mathrm{MPC}}$ & Matriz de ganhos do controlador preditivo \\
\hline $\mathrm{K}_{\mathrm{p}}$ & Ganho do processo \\
\hline$L$ & Número de modelos alternativos do processo \\
\hline $\mathbf{M}$ & Matriz de transição de estado \\
\hline$m$ & Horizonte de controle \\
\hline$n$ & Instante de amostragem futuro $n=1,2, \ldots$ \\
\hline$n a$ & Número de pólos do processo \\
\hline
\end{tabular}




\begin{tabular}{|c|c|}
\hline$n p$ & Horizonte de predição \\
\hline$n u$ & Número de variáveis manipuladas \\
\hline ny & número de variáveis controladas \\
\hline $\mathbf{N}$ & Matriz de correção do erro \\
\hline$n r$ & Horizonte de otimização \\
\hline $\mathbf{N}_{\mathrm{r}}$ & Matriz de predição para controle \\
\hline$\tilde{N}$ & Matriz de propagação dos setpoints das saídas \\
\hline$N_{p}$ & Matriz de repetição das ações de controle \\
\hline$n_{0}$ & Número de translações contínuas das predições \\
\hline$n h$ & Número de instantes esparsos das predições \\
\hline OP & saída do controlador em percentagem \\
\hline$O P_{S S}$ & Saída do controlador no estado estacionário inicial \\
\hline$p_{0}$ & Parâmetro de predição da saída indep. do tempo \\
\hline$p$ & Parâmetro de predição da saída dep. do tempo \\
\hline$p^{i}$ & Vetor de parâmetros indep. de predição das saídas \\
\hline$p^{t}$ & Vetor de parâmetros dep. de predição das saídas \\
\hline$p_{i}^{c}$ & Parâmetro indep. corrigido de predição da saída \\
\hline$p_{i, j, g}^{c}$ & Parâmetro dep. corrigido de predição da saída \\
\hline$r$ & Raiz do modelo do sistema \\
\hline$s$ & Resposta ao degrau unitário \\
\hline $\mathbf{S}$ & Matriz de resposta ao degrau unitário \\
\hline $\mathbf{S}_{m}$ & Matriz dinâmica do sistema \\
\hline $\mathrm{T}$ & Período de amostragem \\
\hline$t$ & Instante de tempo \\
\hline$u$ & Valor da entrada (variável manipulada) \\
\hline$w$ & Desvio da predição em relação à planta \\
\hline$W_{\mathrm{k}}$ & Vetor de perturbação no estado do sistema \\
\hline$y_{d}$ & Resposta ao degrau unitário \\
\hline$y$ & Valor da saída (variável controlada) \\
\hline $\mathrm{y}$ & Vetor da predição da saída \\
\hline$\hat{y}$ & Valor de medida da saída \\
\hline
\end{tabular}




$\begin{array}{ll}y^{c} & \text { Predição corrigida da variável controlada } \\ \tilde{\mathbf{y}} & \text { Vetor de predição da saída para controle } \\ \overline{\mathbf{y}} & \text { Vetor de predição da saída após ação de controle } \\ y_{s p} & \text { Setpoint da controlada } \\ \mathbf{y}_{s p} & \text { Vetor de setpoints das controladas }\end{array}$

Símbolos Gregos
$\Delta$
Incremento de uma variável
$\tau_{\mathrm{D}}$
Tempo derivativo do controlador PID
$\tau_{\mathrm{I}}$
Tempo integral do controlador PID
$\theta$
Tempo morto
$\gamma$
Peso que pune o desvio
$\lambda$
Fator de supressão da manipulada
$\gamma$
Fator de importância da controlada
$\Lambda$
Fatores de supressão das manipuladas
$\Gamma$
Matriz dos fatores de importância das controladas
$\Phi$
Função objetivo do QDMC
$\Psi$
Matriz de predição das saídas
$\gamma$
Resíduo da função objetivo do problema de NLP 


\section{RESUMO}

As colunas depropanizadoras existentes nas refinarias de petróleo têm como função a separação entre as correntes de propano e butano. O objetivo de controle nestas colunas é a especificação de um teor máximo de iso-butano e mais pesados $\left(\mathrm{C}_{4}{ }^{+}\right)$na corrente de propano e do teor máximo de propano e mais leves $\left(\mathrm{C}_{3}{ }^{-}\right)$na corrente de butano. Controladores multivariáveis tradicionais, que normalmente são implementados nas colunas depropanizadoras, apresentam grande dificuldade para manter os produtos dentro de suas especificações, isto se deve ao fato de que este processo apresenta um comportamento bastante não-linear ao longo de toda sua região de operação.

Neste trabalho temos como objetivo estudar as dificuldades encontradas no projeto de controle para esse tipo de sistema e implantar na planta industrial um controlador multivariável utilizando múltiplos modelos para controle da coluna. Para realizarmos este estudo utilizamos o simulador de processos HYSYS ${ }^{\text {TM }}$ para verificarmos o comportamento estático e dinâmico do processo. Os modelos utilizados para representar o processo são aqueles obtidos durante o estudo do comportamento dinâmico. Para implantação do controlador na unidade industrial é utilizado o SICON (Sistema de Controle da Petrobras) sendo algumas de suas rotinas modificadas para permitir a inclusão dos múltiplos modelos. Durante o estudo são comparadas as performances dos controladores QDMC e MMPC (Multi-Model Predictive Control) resolvido através de um algoritmo para NLP (Non Linear Programming).

O controlador multimodelos (MMPC) é apresentado na forma de variáveis de estado podendo controlar sistemas de grande porte, inclusive sistemas com dinâmicas lentas e rápidas. Esta formulação permite prever as variáveis controladas em instantes de tempo esparsos e diferentes para cada controlada. O MMPC é capaz de tratar problemas de controle não-linear usando modelos lineares, introduzindo o conceito de robustez com a utilização do conjunto de modelos. O MMPC exige um menor esforço de sintonia que o QDMC sendo adequado para uma região mais ampla de operação. 


\begin{abstract}
Depropanizer columns are used in oil refineries for the separation of the propane stream from the butane stream. The control objective of these columns is the specification of a maximum content of iso-butane and heavier components $\left(\mathrm{C}^{+}\right)$in the propane product and the maximum content of propane and lighter components $\left(\mathrm{C}^{-}\right)$in the butane product. Multivariable controllers usually implemented in depropanizer columns frequently present great difficulty to maintain the products inside their specification ranges. This deficiency is due to the fact that the process presents a quite non-linear behavior along its operating window.

The objective of the present work is to study the difficulties found in the design of the control system for the aforesaid process, and to implement in an industrial plant a multivariable controller using multiple models for the control of the separation column. To accomplish this study we used the HYSYSTM process simulator to verify the static and dynamic behavior of the process. The models used to represent the real process in the controller are those obtained during the study of the dynamic behavior. The controller implementation in the industrial unit was done with SICON (Control System of Petrobras), which had some of its routines modified to allow the inclusion of multiple models. Along the work, performances of QDMC and MMPC(Multi-Model Predictive Control) controllers were compared. MMPC was solved through an algorithm for NLP (Non Linear Programming).

The Multi-Model (MMPC) controller was implemented using a state space formulation which allows for the implementation of very large systems and besides, systems with simultaneous slow and fast dynamics. This formulation allows to foresee the controlled variables at sparse sample instants, that can be distinct for each controlled variable. MMPC is able to handle non-linear control problems using linear models by introducing the robustness concept with the use of a set of models. MMPC demands a smaller tuning effort than QDMC, and can be adapted to a wide range of operating conditions.
\end{abstract}




\section{1 \\ INTRODUÇÃO}

\section{1 - Apresentação da problemática de controle de colunas de pureza elevada}

As colunas depropanizadoras presentes nas refinarias de petróleo da Petrobras estão localizadas nas unidades de Craqueamento Catalítico Fluido (UFCC's). Estas colunas são utilizadas para permitir a separação das correntes de propano e butano. No caso da RPBC (Refinaria Presidente Bernardes Cubatão) o propano é enviado para o pool de GLP (gás liquefeito de petróleo) e o butano é enviado para a unidade de gasolina de aviação (UGAV).

A estratégia de controle regulatório existente na coluna depropanizadora é feita no SDCD (Sistema Digital de Controle Distribuído) e consiste nos seguintes controladores principais: controlador de vazão de carga (sendo este cascateado pelo controlador de nível do vaso de carga), controlador de pressão da coluna (controla a pressão no estágio 24, manipulando a vazão de retirada de produto de topo (propano)), controlador de nível de fundo (manipula a vazão de retirada de produto de fundo (butano)), controlador de temperatura de fundo (manipula a entrada de fluído de aquecimento (gasóleo pesado) para o refervedor) e por último o controlador de vazão de refluxo (tem a possibilidade de ser cascateado pela temperatura de topo). A atuação do operador para controle da qualidade dos produtos é feita preferencialmente manipulando o set-point do controlador de vazão de refluxo e o set-point do controlador de temperatura de fundo da coluna.

O controlador preditivo proposto neste trabalho tem a seguinte estrutura: duas variáveis controladas que são o teor de $\mathrm{C}^{+}$(componentes mais pesados que o propano) no propano (ou a temperatura de topo) e o teor de $\mathrm{C}^{-}$(componentes mais leves que o propano) no butano, duas variáveis manipuladas que são o set-point da vazão de refluxo e o set-point da vazão de fluido de aquecimento do refervedor e duas variáveis perturbadoras que são a vazão de carga e a temperatura do fluido de aquecimento. A prática mostra que a utilização do teor de $\mathrm{C}^{+}$como variável controlada não é adequada, devido ao modelo (inferência) atualmente disponível para cálculo da mesma não 
apresentar resultados satisfatórios. Assim, neste trabalho, optou-se por utilizar a temperatura de topo da coluna como feito normalmente na planta industrial. No entanto, os modelos de processo levantados para o controlador contemplam as duas variáveis.

Tradicionalmente o projeto do controlador preditivo, consiste em definir inicialmente o problema de controle e as funções do controlador, além da escolha das variáveis manipuladas, controladas e perturbações. Uma vez definidas as funções de controle, identifica-se o processo através de testes em degrau nas variáveis manipuladas e perturbações medidas, em um determinado ponto de operação do processo. Em seguida, constrói-se o controlador configurando e customizando a sua programação. Finalmente, sintoniza-se o controlador fazendo uso de simulação para definir os melhores valores dos parâmetros de sintonia. Uma alternativa para se fazer a sintonia de forma sistemática para sistemas quadrados pode ser encontrada em Trierweiler et al. (2001). A etapa final consiste na interligação do controlador preditivo aos controladores PID do processo, através de um sistema digital, normalmente SDCD (Sistema Digital de Controle Distribuído).

Com o objetivo de estudar melhor os problemas de controle desta natureza, podemos utilizar simuladores estáticos e dinâmicos rigorosos. Com esta ferramenta, podemos efetuar um estudo detalhado do problema de controle, determinando na região de operação das variáveis manipuladas, os ganhos do processo e suas dinâmicas nos diversos pontos de operação. Com este estudo, podemos determinar os limites do sistema quanto à sua capacidade de controle.

\section{2 - Revisão bibliográfica}

Os controladores preditivos (MPC's) são uma classe de algoritmos de controle que calculam uma série de ações das variáveis manipuladas com a finalidade de otimizar o comportamento futuro da saída da planta. As primeiras aplicações industriais foram implementadas nas unidades de processo das refinarias de petróleo.

Os primeiros algoritmos MPCs aplicados industrialmente surgiram nos meados da década de 70, sendo a primeira aplicação industrial apresentada por Richalet em 1976 e reportada em 1978 (Richalet et al., 1978). O controlador foi implementado em uma coluna fracionadora de FCC e também em um sistema de geração de vapor. Este 
algoritmo ficou conhecido como MPHC (Model Predictive Heuristic Control) e também como MAC (Model Algoritmic Control). Paralelamente Cutler e Ramaker desenvolveram o controlador DMC (Dynamic Matrix Control) sem restrições, apresentado em 1979 (Cutler e Ramaker, 1979). Em 1980, Prett e Gillette descreveram uma aplicação do algoritmo DMC sem restrições num conjunto reator/regenerador de FCC (Craqueamento Catalítico). Esta foi a primeira aplicação do controlador preditivo em dois níveis. O primeiro nível consta de um algoritmo de programação linear (LP), que resolve um problema de otimização no estado estacionário sujeito às restrições do processo. O segundo nível recebe os valores ótimos das entradas e os setpoints das saídas e resolve o problema através do controlador DMC sem restrições.

Os controladores MAC e DMC apresentavam dificuldade de incorporar as restrições nos problemas de controle. Este fato levou ao surgimento de uma nova classe de controladores preditivos. O problema foi resolvido com a utilização dos algoritmos QDMC (Quadratic Dynamic Matrix Control) e LDMC (Linear Dynamic Matrix Control), nos quais as restrições aparecem explicitamente dentro do problema de otimização. Em 1985 Morshedi, Cutler e Skrovanek descreveram o algoritmo LDMC e em 1986, Garcia e Morshedi descreveram o algoritmo QDMC e apresentaram os resultados de uma aplicação em um forno de pirólise. $\mathrm{Na}$ abordagem do LDMC e QDMC, foi introduzido o controle por faixa, onde se controla o processo através de uma janela de operação.

Neste estágio de desenvolvimento dos controladores preditivos, a Petrobras decidiu implantar seus próprios controladores, sendo os mesmos desenvolvidos em conjunto com o departamento de engenharia química da Escola Politécnica da USP. Assim, surgiu a primeira aplicação em um forno de resíduo atmosférico da unidade de destilação da Refinaria Presidente Bernardes de Cubatão - RPBC (Zanin e Odloak, 1992), onde se utilizou o controlador LDMC em uma camada. Em seguida surgiram outras aplicações de mesma natureza, como os controles da coluna debutanizadora da REPLAN, da coluna atmosférica da REPLAN (Magalhães e Odloak, 1995) e da coluna de destilação à vácuo da REPLAN (Almeida et al., 1996). Além destas aplicações, surgiram outras nas unidades de craqueamento catalítico fluido, onde se utilizou o controlador DMC em duas camadas. Dentre eles, temos o controle do conversor do FCC da Refinaria do Vale do Paraíba - REVAP (Moro e Odloak, 1994, Moro e Odloak, 
1995) e do conversor do FCC da REPLAN (Spandri et al., 1995). A partir destas aplicações, estes controladores foram amplamente difundidos nas unidades da Petrobras, incluindo unidades de gasolina de aviação, coqueamento retardado, recuperação de aromáticos e outras.

Os controladores DMC, LDMC e QDMC já estavam consolidados em meados da década de 80. A partir daí despertou-se o interesse dos pesquisadores pela análise da robustez dos mesmos, isto é, a utilização da estrutura de controle junto à planta, onde existem incertezas nas medidas e nos modelos utilizados pelo controlador. Uma série de trabalhos exploraram este assunto, dentre eles podemos destacar Garcia e Morari (1982, 1985); Rouhani e Mehra (1982); Morari e Doyle (1986) e Morari e Zafirou (1989). Neste ponto surgiram algumas propostas de análise de estabilidade dos MPC's, entre elas uma abordagem paramétrica dos MPCs, denominado GPC (Generalized Predictive Control) proposto por Clarke et al. em 1987. Esta abordagem estimulou uma uniformização dos MPCs sob a forma de variáveis de estado. Li et al. (1989) apresentam uma descrição bem detalhada do MPC em variáveis de estado, explicando as suas propriedades e apresentando melhorias em relação ao DMC, como a utilização do filtro de Kalman para modelar as perturbações não medidas.

Morari e Lee (1991) propuseram soluções de controle para sistemas de grande porte, onde se utilizou a representação do sistema em espaço de estado, e o modelo da perturbação através do filtro de Kalman e incluiu-se a análise de robustez no projeto do controlador.

Odloak (1995), apresentou o controlador DMC em variáveis de estado, com possibilidade de analisar o sistema de controle em malha fechada, isto é, a resposta do processo às ações de controle é equacionada juntamente com o controlador. Esta abordagem permite a verificação da estabilidade da malha fechada sem realizar as simulações do sistema. Isto possibilita o estudo da estabilidade do controlador diante dos parâmetros de sintonia e de possíveis configurações do controle.

A utilização do controlador MPC tradicional em variáveis de estado tem a dimensão do estado correspondente ao número de coeficientes da resposta ao degrau do modelo do DMC. Assim, as principais limitações do DMC (Lundström et al., 1995), como o esforço computacional para controladores de grandes dimensões, e processos com dinâmicas muito lentas, continuaram sendo restrições ao uso desta abordagem. 
Com objetivo de resolver este tipo de problema, foram apresentadas algumas propostas de truncamento do modelo de resposta ao degrau, entre elas (Hovd et al., 1993), que não teve porém muita aceitação. Outra abordagem é a compactação do controlador através da redução da ordem do modelo. Lee et al. (1994) propuseram uma redução do estado através da construção de um filtro ótimo (filtro de Kalman), cujo ganho é resolvido através da equação de Riccati. Neste último trabalho foi realizada uma conexão desta abordagem com o controlador DMC, porém os autores não conseguiram manter exatamente a forma original do DMC e QDMC.

Com a necessidade de se aplicar os MPC's para sistemas de grande porte (um único controlador para toda uma unidade de processo) ou sistemas com dinâmicas lentas, e diante das dificuldades de apresentação do controlador DMC em sua formulação original em espaço de estado e de forma reduzida, Odloak (1996) apresentou uma forma eficiente do controlador em espaço de estado de ordem reduzida. Nesta formulação, o modelo do processo é apresentado de forma analítica, através de modelos paramétricos contínuos ou discretos. Isto possibilitou a manutenção da forma original do DMC e a utilização de parâmetros contínuos, tais como, tempos mortos fracionários, a possibilidade da utilização de instantes de predição esparsos, podendo até utilizar períodos de amostragem diferentes para cada variável controlada, o que é interessante quando num mesmo controlador temos variáveis que apresentam dinâmicas lentas e outras rápidas. Esta formulação também permitiu a análise de estabilidade dos MPCs sem muito esforço computacional (Gouvêa e Odloak, 1997).

Diante da necessidade de resolver os problemas de controle não-lineares, como o controle de colunas de destilação de alta pureza que é um caso típico em unidades petroquímicas, algumas alternativas de controladores não-lineares foram propostas, onde podemos utilizar modelos fundamentais (baseados em equações diferenciais de conservação de massa e energia e equações constitutivas), modelos empíricos, obtidos através de identificações não-lineares e modelos híbridos, podendo-se até utilizar modelos por redes neurais. Além disso, podemos resolver o problema de controle através de otimização não-linear ou até mesmo a linearização do modelo no ponto de operação e usar um controlador linear. Estas abordagens são descritas por Henson (1998) e Morari e Lee (1999). 
A utilização de controladores multi-modelos é uma alternativa interessante para resolvermos os problemas onde o processo é variante no tempo e no estado. Nesta abordagem resolvemos o problema de controle que satisfaz a todos os possíveis modelos lineares do processo. Este problema de controle pode ser resolvido através do algoritmo LMI (Linear Matrix Inequality). Esta técnica foi motivada por recentes desenvolvimentos na teoria e aplicações em problemas de controle, e na utilização de otimização através de LMI (Boyd te al., 1994). Kothare et al. (1996) apresentaram uma solução de controle MPC usando LMIs, porém não apresentando os modelos alternativos diretamente no problema de controle.

Odloak tem estudado a utilização de MMPCs (Multi-Model Predictive Control), sendo que o conjunto de modelos e a equação de Riccati aparecem inclusas no problema de controle. Neste caso o controlador é projetado com garantia de estabilidade (Rodrigues e Odloak, 1999). Almeida, Rodrigues e Odloak (2000) apresentam uma aplicação de multiplos-modelos utilizando-se LMI's para controle de uma coluna debutanizadora.

\section{3 - Objetivo do trabalho}

Este trabalho tem como objetivos estudar o sistema de controle de uma coluna depropanizadora e implantar em uma unidade industrial um controlador preditivo multivariável com múltiplos modelos (MMPC) sendo este resolvido através de um algoritmo de programação não linear (NLP).

Neste trabalho procuramos fazer um estudo mais detalhado do sistema. Primeiramente, procuramos estudar o comportamento estático do processo utilizando modelos rigorosos, através de simulador. Nesta etapa, define-se a região de operação do sistema e o comportamento das variáveis controladas ao longo desta região. Depois, determinam-se os ganhos do processo ao longo da região de operação, sendo que nesta etapa avaliamos as não-linearidades do sistema. Como resultado desta etapa, avaliam-se as propriedades da matriz de ganhos do processo na região de operação obtida. As propriedades analisadas estão ligadas às características de interações entre as variáveis, condicionamento e determinantes das matrizes de ganhos do processo. A etapa seguinte corresponde à construção do modelo dinâmico no simulador HYSYS ${ }^{\top M}$ e à identificação 
do processo em vários pontos ao longo da região de operação. A identificação é feita utilizando-se procedimento proposto por Odloak (1996) onde os modelos são obtidos na forma analítica. Após a identificação dos modelos construímos o controlador no Matlab $^{\mathrm{TM}}$ e fazemos a simulação considerando um dos modelos como sendo o modelo da planta. Com o controlador construído, fazemos a conversão de todas as rotinas para a linguagem FORTRAN, pois o controlador industrial será montado utilizando-se esta linguagem. Com o controlador na linguaguem FORTRAN poderemos fazer a integração do mesmo com o SICON (Sistema Integrado de Controle) que é uma estrutura para montagem dos controladores preditivos desenvolvida pela Petrobras (veja item 9.2.1). A seguir o controlador é colocado em operação no modo de simulação para se fazerem os ajuste preliminares na sintonia do mesmo. Após esta etapa de simulação o controlador é colocado em operação com atuação ativa sobre as variáveis da unidade industrial. A seguir, são realizados testes para verificar a performance de operação do controlador. 


\section{PROBLEMA DE CONTROLE DA COLUNA DEPROPANIZADORA}

As colunas depropanizadoras são utilizadas em refinarias para se fazer a separação das correntes propano e butanos. Na RPBC a corrente propano é enviada para o pool de GLP e a corrente de butanos enviada para a unidade de gasolina de aviação (UGAV).

O esquema de produção é ilustrado na figura 2.1 .

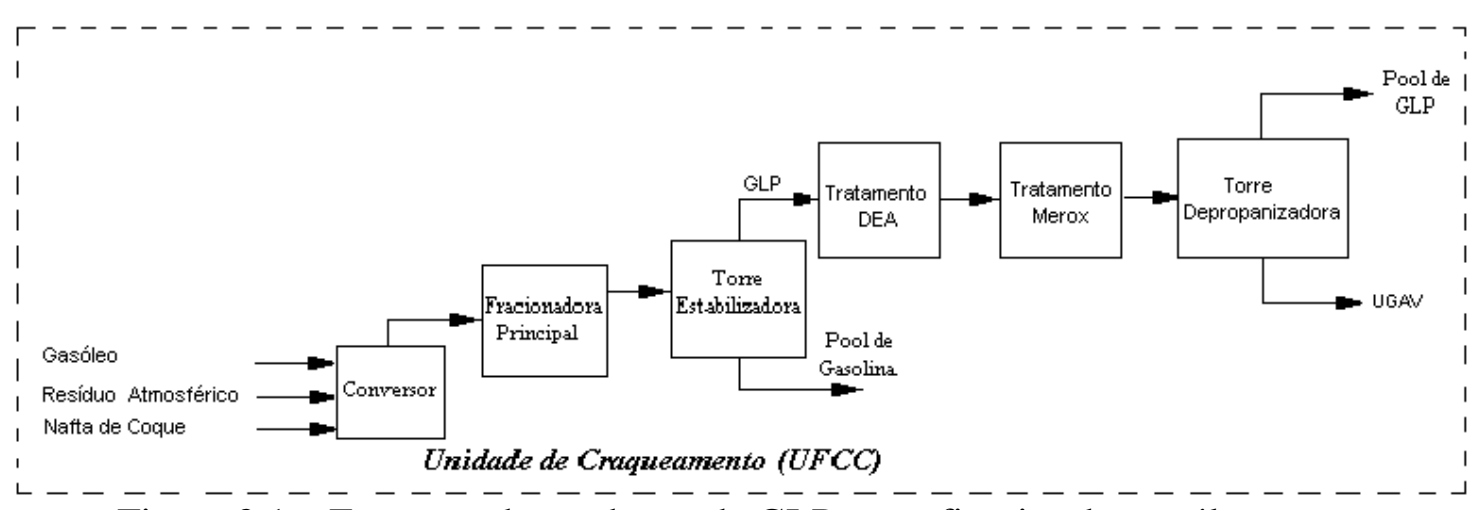

Figura 2.1 - Esquema de produção de GLP em refinarias de petróleo

\section{1 - Especificações dos produtos}

As correntes de propano e butano fracionadas na coluna depropanizadora possuem especificações que devem ser obedecidas rigorosamente.

\subsection{1 - Especificações do propano:}

Uma das especificações mais importantes do propano é o teor de $\mathrm{C}^{+}$ (componentes mais pesados que o propano) e deverá ter um valor de $3 \%$ no máximo. $\mathrm{O}$ objetivo é a minimização das perdas de iso-butano para o pool de GLP, cuja perda é compensada por importação, com o correspondente custo adicional. 


\subsection{2 - Especificações do butano}

No caso da corrente de butano, o teor de $\mathrm{C}^{-}$(componentes mais leves que o butano) é uma de suas principais especificações e deverá ter um valor de 3\% no máximo, sendo o objetivo evitar perda de controle operacional na unidade de gasolina de aviação (UGAV) devido à presença de componentes leves no processo e por questões de segurança no armazenamento da corrente de butanos.

\section{2 - Descrição do processo}

A coluna depropanizadora estudada pertence à unidade de craqueamento catalítico da Refinaria Presidente Bernardes (RPBC), sendo sua carga (GLP) proveniente da seção de tratamento. O tratamento do GLP é feito em duas etapas, ou seja, dois processos diferentes que são: tratamento DEA, no qual uma solução de DEA (Dietanolamina) é utilizada para remoção de contaminantes tais como $\mathrm{CO}_{2}, \mathrm{CO}, \mathrm{H}_{2} \mathrm{~S}$; após este processo temos o tratamento Merox, que consiste na remoção de compostos de enxofre (mercaptans) que são transformados em dissulfetos pela ação de um catalisador em presença de soda caústica. $O$ tratamento tem como finalidade eliminar a corrosividade do GLP.

O GLP após a seção de tratamentos é enviado ao vaso V-01, do qual é bombeado para a coluna depropanizadora T-01, sendo sua vazão controlada através do FC-01, o qual é cascateado pelo controle de nível do V-01. Antes de entrar na coluna, o GLP é pré-aquecido no trocador de calor E-1 trocando calor com a nafta proveniente do fundo da debutanizadora. A temperatura do GLP é mantida constante através da manipulação da vazão de nafta através do trocador E-01. O GLP entra na coluna no estágio 16 (a coluna é composta de 31 pratos valvulados).

Esta carga é destilada sendo os vapores de topo totalmente condensados e subresfriados no E-02 e enviados para o vaso de topo (V-02). Neste vaso, a corrente líquida é dividida em duas sendo uma o refluxo de topo e a outra retirada como destilado para pool de GLP. 
Parte do refluxo interno que atinge o fundo da coluna é vaporizado no refervedor (E-03) e outra parte é retirado como produto de fundo sendo resfriado no E04 e depois enviado para unidade de gasolina de aviação.

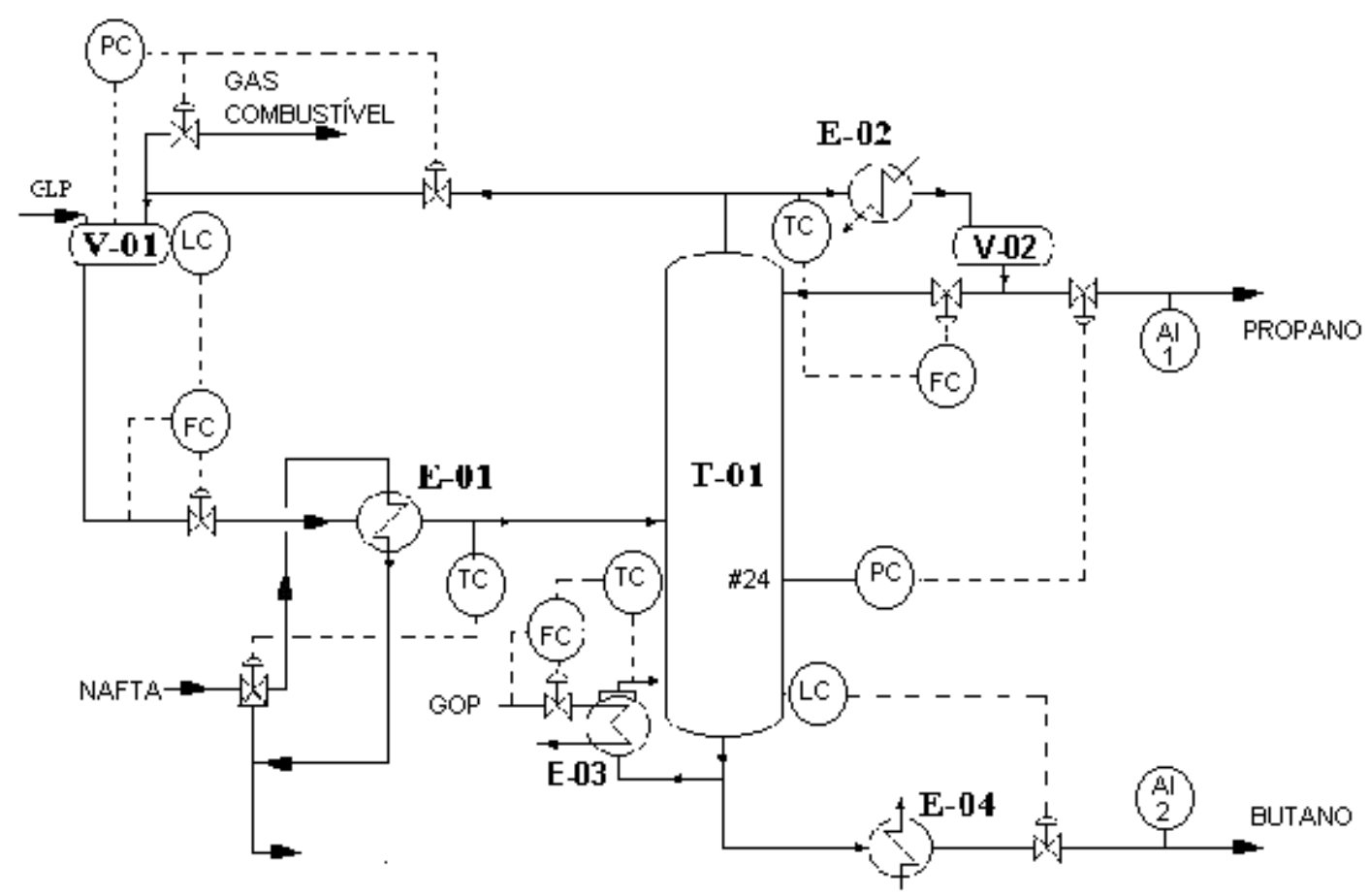

Figura 2.2 - Fluxograma de processo da coluna depropanizadora

\section{3 - Variáveis operacionais}

A coluna depropanizadora separa os componentes voláteis no destilado (propano/propeno) e os componentes pesados no produto de fundo (butanos).

Para garantirmos a especificação dos produtos as seguintes variáveis devem ser observadas:

1-Vazão de carga da coluna;

2-Temperatura da carga da coluna;

3-Composição da carga;

4-Pressão da coluna;

5-Vazão de refluxo;

6- Vazão de fluido de aquecimento para refervedor;

7-Temperatura de topo da coluna. 


\subsection{1 - Vazão de carga}

A vazão de carga não pode ser manipulada diretamente pelo operador, pois esta vazão é resultado do controle de nível do vaso de carga.

Um aumento da vazão de carga provoca o aumento da vazão de fluido de aquecimento do refervedor e um conseqüente aumento da vazão de refluxo para manter a qualidade dos produtos.

\subsection{2 - Temperatura da carga}

Conforme indicado na figura 2.2, existe um controlador de temperatura da carga da coluna no nível regulatório. O set-point desse controlador pode ser manipulado diretamente pelo operador. No entanto é mantido sempre em um valor fixo. Assim, não é uma variável utilizada para controle da coluna.

\subsection{3 - Composição da carga}

A composição da carga não pode ser manipulada diretamente pelo operador, pois a distribuição de componentes na carga é função da natureza da carga introduzida na seção de conversão da unidade e das variáveis operacionais desta seção.

Um aumento da fração de leves na carga, provoca um aumento no rendimento da corrente de propano e conseqüentemente aumento da vazão de refluxo para manter a qualidade dos produtos.

\subsection{4 - Pressão da coluna}

A pressão da coluna também é controlada no nível regulatório e o comportamento dessa malha é muito bom o que leva a hipótese de que essa variável possa ser considerada como constante, só devendo ser alterada quando o resultado desejado não puder ser obtido pela manipulação de outras variáveis. 
Um aumento na pressão da coluna, provoca um aumento de dificuldade na separação entre os leves e pesados. Para que esta separação ocorra de forma adequada, deve-se aumentar a vazão de fluido de aquecimento do refervedor e a vazão de refluxo de topo.

\subsection{5 - Vazão de refluxo}

A vazão de refluxo é uma das variáveis mais importantes a serem manipuladas para garantir a especificação dos produtos. Para cada nível de fracionamento desejado há uma razão de refluxo adequada (relação entre a vazão de refluxo e a vazão de destilado).

Um aumento da vazão de refluxo provoca uma redução da quantidade de frações pesadas no destilado. Portanto para manter a qualidade do produto de fundo, é necessário aumentar a vazão de fluido de aquecimento do refervedor.

O limite inferior da vazão de refluxo está ligado à qualidade da separação e o limite superior está ligado às capacidades do refervedor, do condensador, da bomba de refluxo e à hidráulica da coluna.

\subsection{6 - Vazão de Fluido de Aquecimento para refervedor}

A vazão e a qualidade da corrente de propano dependem da quantidade de vapores condensados no sistema de topo, que por sua vez, depende da vazão de fluido de aquecimento para o refervedor.

Um aumento da vazão de fluido de aquecimento do refervedor ocorrerá um aumento da vaporização e conseqüentemente a necessidade de aumento da vazão de refluxo de topo.

\subsection{7 - Temperatura de topo}

Uma variável alternativa à vazão de refluxo é a temperatura de topo, que quando utilizada manipulará a vazão de refluxo. 
Neste caso, um aumento nesta temperatura provoca um aumento da quantidade de frações pesadas no destilado. Portanto para manter a qualidade do produto é necessário diminuir a vazão de fluido de aquecimento do refervedor.

\section{4 - Fatores de desempenho do sistema}

Para otimização da coluna depropanizadora devemos manipular a vazão de refluxo de topo e a vazão de fluido de aquecimento para o refervedor, observando sempre as restrições do processo.

Em termos econômicos as variáveis manipuladas devem ser utilizadas no sentido de se introduzir o mínimo de energia no refervedor e operar com o mínimo de refluxo interno da coluna.

Os limites de especificação dos produtos são as principais restrições do processo que deverão ser impostas ao sistema de controle.

Assim, o problema de otimização tem a seguinte formulação:

Minimizar: - Vazão de refluxo de topo e

- Vazão de fluido de aquecimento do refervedor

Sujeito a restrições de:

- \% máxima de $\mathrm{C}^{+}$no propano

- \% máxima de C3 no butano

\section{5 - Limitações do processo}

A coluna depropanizadora não apresenta limitações físicas para especificação dos produtos, conseguindo a mesma fracionar toda a produção de GLP da UFCC e também correntes de GLP de outras unidades.

\section{6 - Estratégias de controle da coluna depropanizadora}

Uma coluna de fracionamento com separação de produto de topo e fundo com condensador total e tambor de topo inundado, tem 4 graus de liberdades e portanto 4 
variáveis devem ser especificadas. Note que a vazão de carga é manipulada em função do nível do vaso carga (V-01) e portanto não fornece nenhum grau de liberdade adicional

A forma de utilizar estes graus de liberdade é colocando controladores para especificar estes itens. Há uma série de alternativas de arranjos para o sistema de controle usando os graus de liberdade do sistema. A escolha do melhor arranjo leva em consideração a sensibilidade do processo, a linearidade do comportamento na região de operação e o seu comportamento dinâmico.

Os graus de liberdade são utilizados pelos controladores do nível regulatório e cujos "set-points" dão origem às variáveis manipuladas.

As malhas do nível regulatório normalmente especificadas nesta coluna são:

1. Pressão da coluna (estágio 24).

2. Vazão de refluxo de topo da coluna.

3. Temperatura de retorno do refervedor (cascateando a vazão do fluido de aquecimento para o refervedor).

4. Nível do fundo da coluna.

As perturbações mais importantes deste sistema são:

1. Composição da carga.

2. Vazão de carga da coluna.

3. Temperatura do fluido de aquecimento.

Normalmente, a pressão da coluna é mantida no valor de projeto e o nível de fundo é controlado no nível regulatório. Assim, restam dois graus de liberdade para serem utilizados no controle da coluna. Quando em operação manual, o operador fixa a vazão de refluxo e a temperatura de saída do refervedor. Uma outra alternativa seria especificar uma vazão do fluido de aquecimento para o refervedor em vez da temperatura de saída do refervedor.

\subsection{1 - Estratégia temperatura de retorno do refervedor - vazão de refluxo de topo}


Nesta configuração a temperatura de retorno do refervedor para coluna é utilizada para especificar principalmente o teor de $\mathrm{C}^{-}$no butano, e a vazão de refluxo de topo para especificar preferencialmente o teor de $\mathrm{C}^{+}$no propano.

A especificação mais rigorosa é o teor de $\mathrm{C}^{-}$no butano, pois existe a preocupação com o armazenamento desta corrente e também com a possível perda de controle operacional na unidade de gasolina de aviação caso tenhamos um teor de C3acima dos limites. O controlador de temperatura deveria eliminar as possíveis perturbações do processo para manter a qualidade do butano.

Assim, nesta configuração colocamos os seguintes controladores para utilizar os graus de liberdade como mostra a figura 2.2:

1. Controlador de pressão no estágio 24 manipulando a válvula de retirada de destilado (propano).

2. Controlador de nível de fundo da coluna manipulando a válvula de retirada de butano.

3. Controlador de temperatura de retorno do refervedor manipulando a vazão de fluido de aquecimento.

4. Controlador de vazão de refluxo de topo manipulando a válvula de refluxo de topo.

Esta é a configuração utilizada no controle regulatório normal (feito pelo SDCD) e não será objeto de estudo deste trabalho.

\subsection{2 - Vazão de fluido de aquecimento para refervedor - vazão de refluxo}

Nesta configuração colocamos os seguintes controladores como mostra a figura 2.2:

1. Controlador de pressão no estágio 24 manipulando a válvula de retirada de destilado (propano). Tendo em vista o projeto da unidade que considera o tambor de topo inundado (sem interface líquidovapor), essa é a única configuração possível. 
2. Controlador de nível de fundo da coluna manipulando a válvula de retirada de butano.

3. Controlador de teor de $\mathrm{C}^{-}$no butano (AI2), podendo manipular a vazão de refluxo ou a vazão de fluido de aquecimento.

4. Controlador do teor de $\mathrm{C}^{+}$no propano (AI1) ou da temperatura de topo, podendo manipular a vazão de refluxo ou vazão de fluido de aquecimento.

Visto que a sintonia do controlador da temperatura de retorno do refervedor não é trivial pois depende das condições operacionais, que podem tornar a malha muito lenta, para o estudo desta coluna, optou-se por adotar, no nível regulatório, uma estratégia onde é manipulada a vazão de fluido de aquecimento para o refervedor. 


\section{3}

\section{DESCRIÇÃO SUMÁRIA DO HYSYS}

A crescente demanda por produtos com qualidades cada vez mais rigorosas, tem levado as unidades industriais a operarem seus processos cada vez mais próximos de suas restrições. Isto faz com que a engenharia de controle necessite cada vez mais de modelos em estado-estacionário e dinâmico mais realistas para analisar melhor a estabilidade, robustez e performance dos sistemas de controle dos processos químicos.

Diante deste quadro a utilização de um simulador de processo se torna fundamental, sendo o HYSYS ${ }^{\mathrm{TM}}$ (Hyprotech Ltd) um simulador de processos que combina a operação no estado-estacionário com o dinâmico no mesmo ambiente. Ele possibilita passar facilmente do regime estacionário para o dinâmico e no sentido contrário.

O simulador foi desenvolvido em ambiente Windows e orientado à objetos e eventos, com compatibilidade OLE (Object Link Embeding) onde permite-se que o simulador execute funções de outros programas. O programa também disponibiliza os serviços DDE (Dynamic Data Exchange) onde se pode transferir dinamicamente dados para outros programas, como Matlab ${ }^{\mathrm{TM}}$, e no sentido contrário.

Isto permite ao engenheiro criar aplicações customizadas que acessam partes do HYSYS ${ }^{\mathrm{TM}}$ ou construir interfaces customizadas.

Além destas características, possibilita a utilização de pacotes customizados, tais como:

- Controladores

- Cinéticas de reações

- Modelos de colunas extratoras, filtros e etc.

- Pacotes de propriedades

- Outros

Estas customizações podem ser criadas a partir de programas escritos em linguagem FORTRAN ou C/C++, e utilizando Visual Basic como inferface.

As operações unitárias são modulares e utilizam modelos rigorosos, onde são combinadas com algoritmo de solução não-sequencial. Neste algoritmo as informações 
são processadas e os resultados de alguns cálculos são automaticamente propagados no fluxograma para frente e para trás.

A estrutura modular das operações unitárias significa que podem ser calculadas em ambas direções, utilizando informações de uma corrente de saída para calcular as condições de entrada.

O simulador dinâmico permite verificar o comportamento do processo fora do estado-estacionário, analisando os casos servo e regulatório. Desta forma, o modelo dinâmico pode ser utilizado para fazer estudos de controle de processos.

Assim um controlador preditivo pode ser utilizado junto ao modelo do processo disponibilizado pelo simulador.

O simulador de processos é um ambiente de engenharia que se destina às seguintes aplicações:

- Projeto conceitual de processos químicos

- Projeto básico de processos

- Projeto detalhado de plantas

- Projeto de sistemas de controle

- Otimização de processos

- Treinamento de operadores

- Análise de processos

- Monitoração de performance do processo

Em nosso trabalho utilizamos o HYSYS PROCESS 1.2, versão 1.2.4 de outubro de 1997. 


\section{4 \\ SIMULAÇÃO ESTÁTICA DA DEPROPANIZADORA}

Neste capítulo apresentamos os detalhes da montagem do modelo estático do processo a ser utilizado no estudo de controle. Apresentamos as bases de simulação, a caracterização da carga, a montagem das operações unitárias, convergência da simulação, validação do modelo estático e as definições dos estudos da simulação estática.

Para a montagem do modelo dinâmico do processo no HYSYS ${ }^{\mathrm{TM}}$, inicialmente é necessário construir o modelo em estado estacionário. Tendo o modelo em estado estacionário, é possível estudarmos o comportamento do processo. Desta forma é possível determinar a região de operação, isto é, as possíveis combinações das variáveis independentes que correspondem às situações reais de operação do sistema. Podemos também avaliar as não-linearidades dos ganhos do processo, que são importantes para uma melhor avaliação do comportamento do sistema de controle.

\section{1 - Etapas da construção do modelo estático}

Os seguintes aspectos devem ser verificados quando da elaboração do modelo estacionário a ser analisado:

$\checkmark$ Definição das bases de simulação;

$\checkmark$ Caracterização da carga do sistema;

$\checkmark$ Definição das operações unitárias envolvidas no processo;

$\checkmark$ Especificação dos graus de liberdade do sistema;

$\checkmark$ Validação do modelo estático;

$\checkmark$ Definição dos estudos da simulação estática.

Nos próximos itens apresentamos a sequiência para construção do modelo estático de acordo com os itens acima.

\subsection{1 - Definição das premissas da simulação}


Nos simuladores, inicialmente definimos as bases de simulação que consiste em definir o conjunto de equações para os cálculos de equilíbrio, propriedades físicoquímicas e termodinâmicas, e o conjunto de componentes químicos que serão utilizados na simulação.

Na definição do modelo de propriedades das substâncias envolvidas no sistema em estudo, especificamos a natureza química dos componentes químicos da simulação. Para o nosso caso onde os hidrocarbonetos são apolares, o modelo termodinâmico pode ser representado por equações de estado. Neste trabalho foi utilizado o modelo desenvolvido por Peng e Robinson (1976), que prevê com boa precisão as propriedades para estes tipos de hidrocarbonetos.

Com o modelo de propriedades das substâncias envolvidas na simulação definido, caracterizamos os componentes presentes no sistema. Para a depropanizadora, as correntes envolvidas no sistema são constituídas basicamente de hidrocarbonetos puros.

Os componentes utilizados na simulação podem ser caracterizados através de cromatografia gasosa e estão listados na tabela 4.1

\begin{tabular}{|l|l|l|l|l|}
\hline Componente & Símbolo & Fórmula & $\begin{array}{l}\text { Ponto de } \\
\text { ebulição (oC) }\end{array}$ & $\begin{array}{l}\text { Massa } \\
\text { molecular }\end{array}$ \\
\hline metano & $\mathrm{C} 1$ & $\mathrm{CH} 4$ & -161.52 & 16.04 \\
\hline etileno & $\mathrm{C} 2=$ & $\mathrm{C} 2 \mathrm{H} 4$ & -103.75 & 28.05 \\
\hline etano & $\mathrm{C} 2$ & $\mathrm{C} 2 \mathrm{H} 6$ & -88.60 & 30.07 \\
\hline propeno & $\mathrm{C} 3=$ & $\mathrm{C} 3 \mathrm{H} 6$ & -47.75 & 42.08 \\
\hline propano & $\mathrm{C} 3$ & $\mathrm{C} 3 \mathrm{H} 8$ & -42.10 & 44.10 \\
\hline iso-butano & $\mathrm{i}-\mathrm{C} 4$ & $\mathrm{C} 4 \mathrm{H} 10$ & -11.73 & 58.12 \\
\hline iso-Buteno & $\mathrm{i}-\mathrm{C} 4=$ & $\mathrm{C} 4 \mathrm{H} 8$ & -6.85 & 56.11 \\
\hline 1,3-butadieno & 13C4=- & $\mathrm{C} 4 \mathrm{H} 6$ & -4.45 & 54.09 \\
\hline n-butano & n-C4 & C4H10 & -0.50 & 58.12 \\
\hline trans-buteno & tr2-C4= & C4H8 & 0.88 & 56.11 \\
\hline iso-pentano & i-C5 & C5H12 & 27.88 & 72.15 \\
\hline n-pentano & n-C5 & C5H12 & 36.06 & 72.15 \\
\hline n-hexano & n-C6 & C6H14 & 68.73 & 86.18 \\
\hline
\end{tabular}

Tabela 4.1 - Componentes puros considerados 


\subsection{2 - Caracterização da carga do sistema}

Com as bases de simulação definidas passamos para a fase de caracterização da carga do sistema. Esta etapa consiste em definir as condições de processo, como pressão, temperatura e vazão, bem como a sua composição.

$\mathrm{Na}$ planta industrial não é possível caracterizar a carga da coluna depropanizadora diretamente. Assim, a caracterização da carga é feita a partir dos produtos da coluna. Os produtos são analisados separadamente e anotam-se as suas respectivas vazões volumétricas. Portanto, a carga (GLP) é composta da combinação das correntes propano e butano. Serão utilizadas duas composições de carga distintas, retratando perfis de conversão diferentes no sistema de conversão da unidade. As condições operacionais (temperatura, pressão e vazão) da carga serão as mesmas para os dois perfis de composição.

A carga foi composta nas seguintes condições, a partir de dados da planta:

\begin{tabular}{|l|l|l|l|}
\hline Corrente & Vazão & Temp. & Pressão \\
\hline Carga & $2500 \mathrm{~m}^{3} / \mathrm{d}$ & $75{ }^{\circ} \mathrm{C}$ & $19.00 \mathrm{~kg}_{\mathrm{f}} / \mathrm{cm}^{2} \_$man \\
\hline
\end{tabular}

Tabela 4.2 - Condições da carga

A composição da carga foi obtida através da cromatografia dos dois produtos como mostra a tabela a seguir:

\begin{tabular}{|c|c|c|}
\hline Componente & $\%$ Vol. carga 1 & \% Vol. carga 2 \\
\hline metano & 0.8921 & 0.9357 \\
\hline etileno & 0.4956 & 0.4678 \\
\hline etano & 0.4956 & 0.4678 \\
\hline propeno & 24.1975 & 28.4370 \\
\hline propano & 14.8409 & 15.5408 \\
\hline iso-butano & 16.2137 & 13.4214 \\
\hline iso-buteno & 18.5411 & 19.6506 \\
\hline 1,3-butadieno & 0.4654 & 0.8243 \\
\hline n-butano & 9.7452 & 7.0021 \\
\hline trans-buteno & 13.7693 & 13.1535 \\
\hline iso-pentano & 0.2861 & 0.0210 \\
\hline n-pentano & 0.0286 & 0.0781 \\
\hline n-hexano & 0.0289 & 0.0000 \\
\hline
\end{tabular}

Tabela 4.3 - Composição da carga 


\subsection{3 - Definição das operações unitárias envolvidas no processo}

Com a carga do sistema caracterizada, iniciamos a construção das operações unitárias e das correntes envolvidas no sistema como mostrado na figura 4.1.

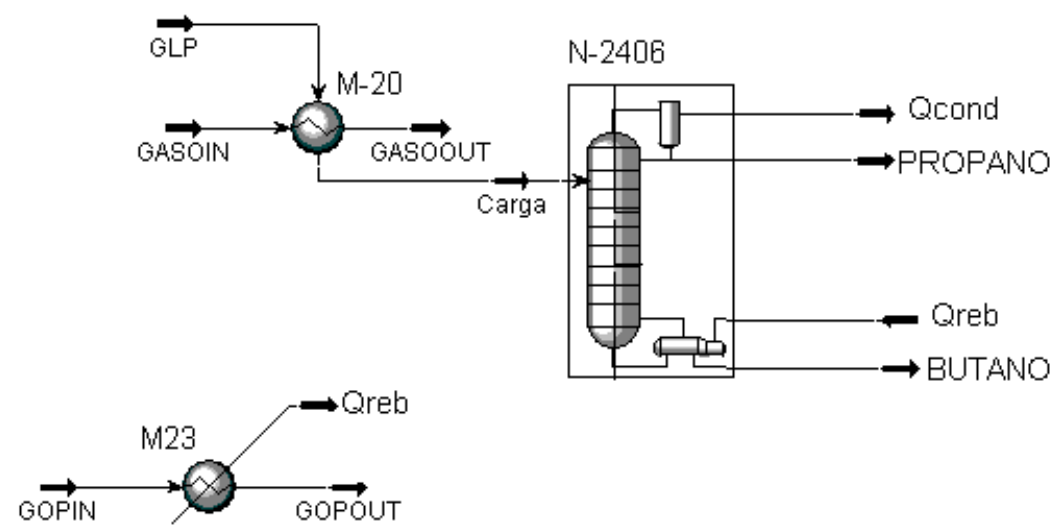

Figura 4.1 - Fluxograma de processo da simulação

Após aquecimento no trocador de calor M20, a corrente de carga é introduzida no sistema à $75^{\circ} \mathrm{C}$ e $19 \mathrm{kgf} / \mathrm{cm} 2 \mathrm{man}$. $\mathrm{O}$ trocador de calor é do tipo casco e tubo em contra-corrente, onde a corrente de carga troca calor com a corrente nafta de fundo da coluna debutanizadora (estabilizadora de gasolina). A perda de carga adotada é no casco de $0.10 \mathrm{kgf} / \mathrm{cm} 2 \mathrm{man}$ e nos tubos de $0.14 \mathrm{kgf} / \mathrm{cm} 2 \mathrm{man}$.

As temperaturas de entrada e saída do lado do casco, e saída do lado dos tubos são conhecidas. As vazões envolvidas também são conhecidas. O UA do trocador é calculado e apresenta um valor de $3.5 \times 10^{4} \mathrm{kcal} /\left({ }^{\circ} \mathrm{C} / \mathrm{h}\right.$ ) (o valor de projeto do trocador é de $\left.11 \times 10^{4} \mathrm{kcal} /\left({ }^{\circ} \mathrm{C} / \mathrm{h}\right)\right)$.

A carga aquecida é introduzida no estágio 16 da coluna N-2406, como mostra a figura 4.2. 


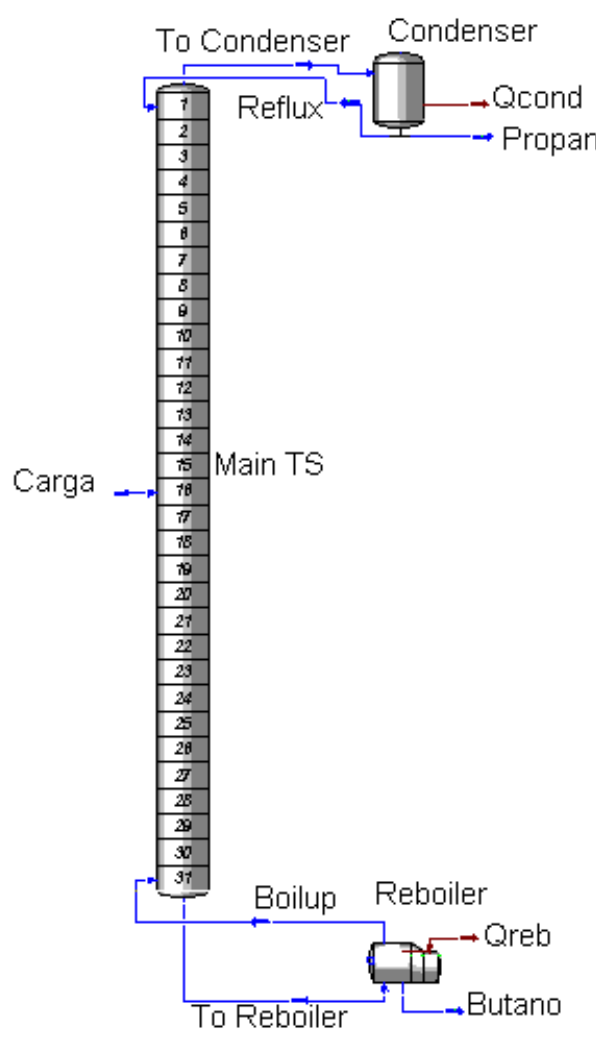

Figura 4.2 - Fluxograma de processo coluna simulada

A coluna tem 31 estágios de equilíbrio com $80 \%$ de eficiência de Murphree levantada experimentalmente. A perda de carga na coluna é de $0.63 \mathrm{kgf} / \mathrm{cm} 2 \mathrm{man}$, com pressão no topo de $18.70 \mathrm{kgf} / \mathrm{cm} 2 \mathrm{man}$ e no prato 24 de $19.18 \mathrm{kgf} / \mathrm{cm} 2 \mathrm{man}$. As pressões são manométricas cuja unidade é; $\mathrm{kgf} / \mathrm{cm} 2 \mathrm{man}$.

O perfil de temperatura tem os seguintes pontos básicos: a temperatura do estágio de topo da coluna é $47^{\circ} \mathrm{C}$, do fundo de $106^{\circ} \mathrm{C}$.

O condensador da coluna é total, ou seja, os vapores do topo são condensados e subresfriados a $32{ }^{\circ} \mathrm{C}$, não apresentando corrente gasosa como produto de topo. As correntes de saída do condensador são: o propano (destilado) e o refluxo de topo. Além destas duas correntes materiais, temos uma corrente de energia que é o calor absorvido na condensação (QCond). Nestas condições a vazão de refluxo de topo é de $4000 \mathrm{~m}^{3} / \mathrm{d}$. Este condensador de topo, na realidade é um conjunto condensador e vaso.

No fundo da coluna, temos um refervedor onde parte do líquido do fundo é vaporizado para fornecer energia ao processo de separação. O líquido do último estágio é introduzido todo no refervedor. A parte vaporizada volta para a coluna abaixo do 
último estágio, sendo a fase líquida removida como produto de fundo. O líquido do último estágio é vaporizado à $106^{\circ} \mathrm{C}$ através do calor cedido (Qreb) de $7.85 \times 10^{6} \mathrm{kcal} / \mathrm{h}$ que corresponde a uma vazão de fluido de aquecimento de $2090 \mathrm{~m}^{3} / \mathrm{d}$.

Nas colunas industriais, a corrente líquida de produto de fundo não passa pelo refervedor. Entretanto considerar que este líquido participa do equilíbrio não representa uma diferença perceptível em relação ao processo real. Portanto, esta é uma simplificação que não causa desvios nos resultados da simulação.

Foi utilizada uma outra operação unitária M23 que é uma simulação do refervedor para obtermos os valores de vazão do fluido de aquecimento (gasóleo pesado). Dentro do ambiente de simulação da coluna calculamos a fração de chave pesado do propano, que corresponde a quantidade de componentes de 4 átomos de carbono ou mais, chamado de $\mathrm{C}^{+}$, e também o chave leve do butano, que corresponde a componentes de 3 átomos de carbono ou menos, chamado C3.

Neste estudo o $\mathrm{C}^{+}$é composto de:

$$
C 4^{+}=i C_{4}+n C_{4}+i C_{4=}+1,3 C_{4==}+\operatorname{trans} C_{4=}+n C_{5}+i C_{5}+n C_{6}
$$

Para o $\mathrm{C}^{-}$temos:

$$
C 3^{-}=C_{3}+C_{3=}+C_{2}+C_{2=}+C_{1}
$$

\subsection{4 - Especificação dos graus de liberdade para simulação do sistema}

Como já visto, para tornar o modelo do sistema unívoco em termos de condições operacionais, devemos especificar os graus de liberdade do sistema. Inicialmente devemos especificar as características da carga como: temperatura, pressão e composição da carga. Ao passar pelo pré-aquecedor de carga, temos que especificar a perda de calor pelas paredes do trocador de calor (no caso é zero) e o produto UA do trocador ou as temperaturas das correntes. Além disso devemos especificar as perdas de carga no casco e nos tubos, ou as pressões das correntes. A carga pré-aquecida entra na coluna de destilação. 
A coluna depropanizadora no estado estacionário apresenta dois graus de liberdade além da pressão, que devem ser especificados conforme as variáveis manipuladas da estratégia de controle. Para simulação da coluna, adota-se a seguinte simplificação: a água de refrigeração para o condensador é manipulada visando manter constante a temperatura de subresfriamento do líquido que sai do condensador. Neste caso, especificamos esta temperatura na simulação. Outras variáveis manipuladas especificadas são: a carga térmica do refervedor e a vazão de refluxo de topo. Como visto, além dessas especificações, devemos definir o perfil de pressões da coluna. $\mathrm{Na}$ tabela 4.4 apresentamos as especificações para o caso base.

\begin{tabular}{|l|l|c|}
\hline $\begin{array}{c}\text { Operação unitária } \\
\text { ou corrente }\end{array}$ & \multicolumn{1}{|c|}{ Especificação } & Valor \\
\hline M-20 & Perda de calor $(\mathrm{MMcal} / \mathrm{h})$ & 0.00 \\
\hline & Temp. ent. casco $\left({ }^{\circ} \mathrm{C}\right)$ & 42 \\
\hline & Temp. sai casco $\left({ }^{\circ} \mathrm{C}\right)$ & 75 \\
\hline & Temp. sai tubos $\left({ }^{\circ} \mathrm{C}\right)$ & 103 \\
\hline & $\Delta$ P no casco $\left(\mathrm{kg}_{\mathrm{f}} / \mathrm{cm}^{2}\right)$ & 0.1 \\
\hline $\mathrm{N}-2406$ & $\Delta \mathrm{P}$ nos tubos $\left(\mathrm{kg} / \mathrm{cm}^{2}\right)$ & 0.14 \\
\hline & Subresfriam.cond. $\left({ }^{\circ} \mathrm{C}\right)$ & 32 \\
\hline & Carga térm. reb $(\mathrm{MMcal} / \mathrm{h})$ & 7.85 \\
\hline & Vazão refluxo topo $\left(\mathrm{m}^{3} / \mathrm{d}\right)$ & 4000 \\
\hline & $\Delta \mathrm{P}$ condensador $\left(\mathrm{kg} / \mathrm{cm}{ }^{2}\right)$ & 0.07 \\
\hline & Pressão topo $\left(\mathrm{kgf} / \mathrm{cm}^{2} \mathrm{man}\right)$ & 18.63 \\
\hline & Pressão fundo $\left(\mathrm{kgf} / \mathrm{cm}^{2} \mathrm{man}\right)$ & 19.33 \\
\hline & $\Delta \mathrm{P}$ refervedor $\left(\mathrm{kg} / \mathrm{cm}^{2}\right)$ & 0.0 \\
\hline
\end{tabular}

Tabela 4.4 - Especificações da simulação

\subsection{5 - Validação do modelo estático}

Com o caso base convergido, devemos validar o modelo construído. Para validarmos o modelo devemos fazer as seguintes verificações:

$\checkmark$ Verificação do balanço material 
$\checkmark$ Verificação do perfil de temperaturas

$\checkmark$ Verificação da qualidade dos produtos

$\checkmark$ Verificação das cargas térmicas do condensador e refervedor

No ajuste do modelo, elegemos os parâmetros a serem manipulados, tais como, eficiências dos estágios, modelo de equilíbrio, composição da carga, entre outros. Na tabela 4.5 apresentamos os resultados do ajuste do modelo para o caso base.

\begin{tabular}{|l|c|c|}
\hline \multicolumn{1}{|c|}{ Variável } & Valor real & Valor simulado \\
\hline Vazão de carga & $2500 \mathrm{~m}^{3} / \mathrm{d}$ & $2500 \mathrm{~m}^{3} / \mathrm{d}$ \\
\hline Vazão de propano & $1012.5 \mathrm{~m}^{3} / \mathrm{d}$ & $1041 \mathrm{~m}^{3} / \mathrm{d}$ \\
\hline Vazão de butano & $1487.5 \mathrm{~m}^{3} / \mathrm{d}$ & $1459 \mathrm{~m}^{3} / \mathrm{d}$ \\
\hline Temperatura carga aquecida & $75 .{ }^{\circ} \mathrm{C}$ & $75^{\circ} \mathrm{C}$ \\
\hline Temperatura de refluxo & $32^{\circ} \mathrm{C}$ & $31.8^{\circ} \mathrm{C}$ \\
\hline Temperatura de topo & $47^{\circ} \mathrm{C}$ & $46.4^{\circ} \mathrm{C}$ \\
\hline Temperatura de fundo & $106^{\circ} \mathrm{C}$ & $106.4^{\circ} \mathrm{C}$ \\
\hline C4 ${ }^{+}$no propano & $2.51 \% \mathrm{vol}$. & $2.45 \%$ vol. \\
\hline C3 ${ }^{-}$no butano & $0.50 \%$ vol. & $0.46 \%$ vol. \\
\hline Vazão de refluxo & $4000 \mathrm{~m} / \mathrm{d}$ & $4000 \mathrm{~m}^{3} / \mathrm{d}$ \\
\hline Carga térmica do refervedor & $7.85 \mathrm{MMcal} / \mathrm{h}$ & $7.85 \mathrm{Mmcal} / \mathrm{h}$ \\
\hline
\end{tabular}

Tabela 4.5 - Resultados da simulação

A vazão de propano para o caso base ficou em $1041 \mathrm{~m}^{3} / \mathrm{d}$ e a de butano em 1459 $\mathrm{m}^{3} / \mathrm{d}$. Na planta industrial, temos uma vazão de propano de $1012.5 \mathrm{~m}^{3} / \mathrm{d}$ e de butano de $1487.5 \mathrm{~m}^{3} / \mathrm{d}$. Tal diferença pode ser uma decorrência de problemas de calibração dos instrumentos de medição e não é motivo de preocupação pois os perfis de composição e temperatura estão em bom acordo com os dados da planta.

Uma boa medida para o desempenho do processo de separação é a verificação do perfil de temperaturas ao longo da coluna. No entanto, a medição da temperatura em todos os estágios na planta industrial não é economicamente viável, portanto colocam-se sensores de temperatura somente nos pontos importantes. Na coluna depropanizadora, os pontos importantes são a temperatura de topo e do fundo da coluna, que apresentaram valores bastante coerentes com os da planta (cf. tabela 4.5). 
Quanto à qualidade dos produtos na simulação do caso base, obtivemos um propano com um teor de $\mathrm{C}^{+}$de $2.45 \%$ vol., e para o butano o teor de $\mathrm{C}^{-}$ficou em 0.46 $\%$ vol., sendo estes valores bastante coerentes com os valores da planta industrial.

\subsection{6 - Definição dos estudos da simulação estática}

Com a simulação estática ajustada, poderemos dar início ao estudo de casos. Neste estudo procuramos determinar a região de operação da coluna em função das suas variáveis independentes, sujeitas às restrições de operação, tais como qualidade dos produtos, hidráulica da coluna e outras.

Em nosso estudo foram manipuladas a vazão de refluxo e a carga térmica do refervedor. As restrições observadas foram o teor de $\mathrm{C}^{+}$no propano, teor de $\mathrm{C}^{-}$no butano, temperatura máxima e mínima no topo e fundo da coluna e o limite de convergência do processo. Nas tabelas 4.6 e 4.7 apresentamos estes valores.

\begin{tabular}{|l|l|l|l|}
\hline Variável independente & Limite mínimo & Limite máximo & Passo \\
\hline Vazão refluxo & $2300 \mathrm{~m}^{3} / \mathrm{d}$ & $4000 \mathrm{~m}^{3} / \mathrm{d}$ & $20 \mathrm{~m}^{3} / \mathrm{d}$ \\
\hline C. term. refervedor & $5.00 \mathrm{MMcal} / \mathrm{h}$ & $8.0 \mathrm{MMcal} / \mathrm{h}$ & $0.02 \mathrm{MMcal} / \mathrm{h}$ \\
\hline
\end{tabular}

Tabela 4.6 - Variáveis manipuladas

\begin{tabular}{|l|l|l|}
\hline Restrição & Limite mínimo & Limite máximo \\
\hline Teor de $\mathrm{C}^{+}$ & 0 & $5 \%$ vol. \\
\hline Teor de $\mathrm{C}^{-}$ & 0 & $5 \%$ vol. \\
\hline Temperatura topo & $44^{\circ} \mathrm{C}$ & $51^{\circ} \mathrm{C}$ \\
\hline Temperatura fundo & $103.5^{\circ} \mathrm{C}$ & $107.5^{\circ} \mathrm{C}$ \\
\hline C. term. condensador & Não tem & $10.95 \mathrm{MMcal} / \mathrm{h}$ \\
\hline C. term. refervedor & Não tem & $8.32 \mathrm{MMcal} / \mathrm{h}$ \\
\hline
\end{tabular}

Tabela 4.7 - Restrições do processo

A sensibilidade do processo é avaliada nesta etapa do estudo de casos, sendo utilizado para isto a avaliação dos ganhos estáticos do processo. Também, podemos 
analisar o comportamento do processo, verificando as não-linearidades do processo, bem como avaliarmos as suas variáveis importantes. No capítulo 6 faremos uma descrição do estudo de casos e comportamento do processo analisado. 


\section{5}

\section{SIMULAÇÃO DINÂMICA DA DEPROPANIZADORA}

A construção do modelo dinâmico no HYSYS ${ }^{\top M}$ é uma etapa realizada após a simulação estática, ou seja, uma vez que o modelo físico-químico estático esteja representando a realidade da planta, é necessário adicionar outras variáveis e operações que afetam a dinâmica do processo, além das variáveis da simulação estática (que requer somente composições, temperatura, vazões, etc.).

Assim, para realizar a simulação dinâmica, é necessário definir os "holdups" (acúmulos) dos equipamentos, as dimensões das válvulas de controle e os controladores do nível regulatório.

Há uma série de etapas a serem cumpridas na construção do modelo dinâmico do HYSYS ${ }^{\text {TM }}$, onde as seguintes são particularmente importantes:

$\checkmark$ Obtenção da solução do modelo estático;

$\checkmark$ Estabelecimento do esquema de controle para o processo;

$\checkmark$ Definição das características dinâmicas das operações unitárias;

$\checkmark$ Definição dos controladores e válvulas de controle do sistema;

$\checkmark$ Chaveamento da simulação do modo estático para o dinâmico.

A seguir apresentaremos as etapas da construção do modelo dinâmico de acordo com a seqüência acima descrita. Na estrutura dos simuladores, inicialmente devemos solucionar o problema de simulação estática. Para isto, devemos seguir o procedimento descrito no capítulo anterior.

Uma vez obtido o modelo estático, devemos definir as variáveis manipuladas e perturbações (independentes) e as variáveis controladas (dependentes) do esquema de controle do sistema, na forma de controle multimalhas. Deve-se tomar o cuidado de manter a coerência entre as especificações na simulação estática e as especificações das variáveis manipuladas dos controladores para a simulação dinâmica. Também é necessário definir as características dinâmicas das operações unitárias envolvidas na simulação. Por exemplo os acúmulos de líquidos, incluindo volumes dos pratos da coluna, dos vasos, trocadores de calor e também o "set-point" do nível fundo da coluna. Assim, para a simulação aqui estudada, definimos as características apresentadas na tabela 5.1. 


\begin{tabular}{|l|l|l|}
\hline \multicolumn{1}{|c|}{ Equipamento } & \multicolumn{1}{|c|}{ Característica } & \multicolumn{1}{c|}{ Valor } \\
\hline Coluna & Diâmetro da coluna & $3.050 \mathrm{~m}$ \\
\hline $\mathrm{N}-2406$ & Comprimento do vertedor & $2.52 \mathrm{~m}$ \\
\hline & Altura do vertedor & $0.08 \mathrm{~m}$ \\
\hline & Volume mínimo & $0.58 \mathrm{~m}^{3}$ \\
\hline Condensador M2422A/C & Volume do condensador & $18.44 \mathrm{~m}^{3}$ \\
\hline O-2407 & Volume do vaso & $16.22 \mathrm{~m}^{3}$ \\
\hline Refervedor & Volume de fundo da torre e refervedor & $8.66 \mathrm{~m}^{3}$ \\
\hline M-2423 & SP do nível de fundo & $50 \%$ \\
\hline
\end{tabular}

Tabela 5.1 - Dados geométricos dos equipamentos

Após definirmos os parâmetros geométricos do sistema, devemos estabelecer as estruturas de controle e as características dos controladores. Isto significa definir as variáveis manipuladas e controladas de cada malha de controle e os seus parâmetros de configuração e de sintonia.

Temos a seguinte estrutura representada na figura 5.1.

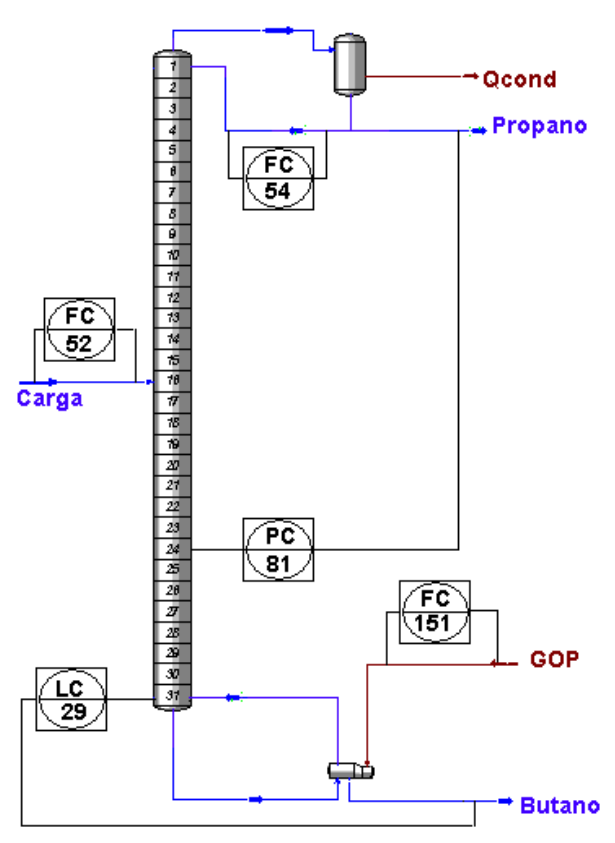

Figura 5.1 - Esquema de controle da coluna depropanizadora. 
O controlador FC-151 é um controlador de carga térmica do refervedor.

A tabela 5.2 mostra os parâmetros dos controladores e válvulas para a estratégia de controle adotada.

\begin{tabular}{|c|c|c|c|c|c|c|c|}
\hline \multirow[t]{2}{*}{ Malha } & \multicolumn{3}{|c|}{ Variável controlada } & \multicolumn{3}{|c|}{ Variável manipulada } & \multirow[t]{2}{*}{ Ação } \\
\hline & Nome & Min. & Max. & $\begin{array}{l}\text { No } \\
\text { me }\end{array}$ & Min. & Max. & \\
\hline FC-54 & $\mathrm{V}_{\mathrm{RF}}$ & 0 & $7000 \mathrm{~m}^{3} / \mathrm{d}$ & $\mathrm{V}_{\mathrm{RF}}$ & 0 & $7000 \mathrm{~m}^{3} / \mathrm{d}$ & Reversa \\
\hline PC-81 & $\mathrm{P}_{\# 24}$ & 7 & $25 \mathrm{kgf} / \mathrm{cm} 2 \mathrm{man}$ & $\mathrm{V}_{\mathrm{PP}}$ & 0 & $3000 \mathrm{~m}^{3} / \mathrm{d}$ & Direta \\
\hline FC-151 & $\mathrm{Q}_{\mathrm{REB}}$ & 0 & $10 \mathrm{MMcal} / \mathrm{h}$ & $\begin{array}{l}\mathrm{Q}_{\mathrm{RE}} \\
\mathrm{B}\end{array}$ & 0 & $10 \mathrm{MMcal} / \mathrm{h}$ & Reversa \\
\hline LC-29 & $\mathrm{L}_{\mathrm{REB}}$ & 0 & $100 \%$ & $\begin{array}{l}\mathrm{V}_{\mathrm{BU}} \\
\text { TANO }\end{array}$ & 0 & $3000 \mathrm{~m}^{3} / \mathrm{d}$ & Direta \\
\hline FC-52 & $\mathrm{v}_{\text {CARGA }}$ & 0 & $100 \%$ & $\begin{array}{l}\mathrm{v}_{\mathrm{CA}} \\
\mathrm{RGA}\end{array}$ & 0 & $5000 \mathrm{~m}^{3} / \mathrm{d}$ & Direta \\
\hline
\end{tabular}

Tabela 5.2 - Parâmetros de configuração dos controladores

Sendo:

$\mathrm{V}_{\mathrm{RF}} \quad$ - Vazão de refluxo de topo;

$\mathrm{P}_{\# 24} \quad-$ Pressão no estágio 24;

$\mathrm{Q}_{\mathrm{REB}} \quad$ - Carga térmica do refervedor;

LREB - Nível de fundo da coluna;

$\mathrm{V}_{\text {BUTANO }}$ - Vazão de butano;

$\mathrm{V}_{\mathrm{PP}} \quad-$ Vazão de propano;

V

\begin{tabular}{|l|c|c|c|}
\hline Malha & \multicolumn{3}{|c|}{ Parâmptros de sintonia } \\
\hline & $K_{c}$ & $\tau_{\mathrm{i}}$ & $\tau_{\mathrm{d}}$ \\
\hline FC-54 & 0.75 & 0.25 & 0.0 \\
\hline PC-81 & 0.35 & 1.35 & 0.0 \\
\hline FC-151 & 0.8 & 0.1 & 0.0 \\
\hline LC-29 & 1.0 & 0.2 & 0.0 \\
\hline FC-52 & 1.0 & 0.1 & 0.0 \\
\hline
\end{tabular}

Tabela 5.3 - Parâmetros de sintonia dos controladores 
O sistema de controle do HYSYS ${ }^{\mathrm{TM}}$ pode ser representado pela estrutura da malha fechada na figura 5.2. A equação do controlador (apresentada na seção 13.4.3 página 425 do manual de referência na sua versão 1.0) tem a seguinte forma:

$$
O P(t)=O P_{S S}+K_{C} E(t)+\frac{K_{C}}{\tau_{I}} \int E(t) d t+K_{C} \tau_{D} \frac{d E(t)}{d t}
$$

sendo:

$\begin{array}{lll}O P(t) & - & \text { Saída do controlador no instante t } \\ O P_{S S} & - & \text { Saída do controlador no estado estacionário inicial } \\ E(t) & - & \text { Erro no instante } \mathrm{t} \\ K_{C} & - & \text { Ganho proporcional do controlador } \\ \tau_{I} & - & \text { Tempo integral do controlador } \\ \tau_{D} & - & \text { Tempo derivativo do controlador }\end{array}$

O erro deve ser definido tendo como referência a ação reversa do controlador, ou seja, se a PV ultrapassa o SP para cima, a saída do controlador (OP) deve decrescer. Portanto, neste caso:

$$
E(t)=S P(t)-P V(t)
$$

sendo:

SP - Setpoint da variável controlada

PV - Variável de processo da variável controlada

No equacionamento do bloco de elemento final de controle da figura 5.2, devemos aplicar o modelo da válvula para a fase líquida ou vapor, conforme o caso. A equação da válvula de controle para a fase líquida é dada por:

$$
F=\frac{O P \%}{100}\left(F_{\text {max }}-F_{\text {min }}\right)+F_{\text {min }}
$$

sendo:

F - Vazão através da válvula de controle

OP\% - saída do controlador em percentagem

$F_{\max }$ - Vazão máxima através da válvula de controle 
$F_{\min } \quad$ - Vazão mínima através da válvula de controle

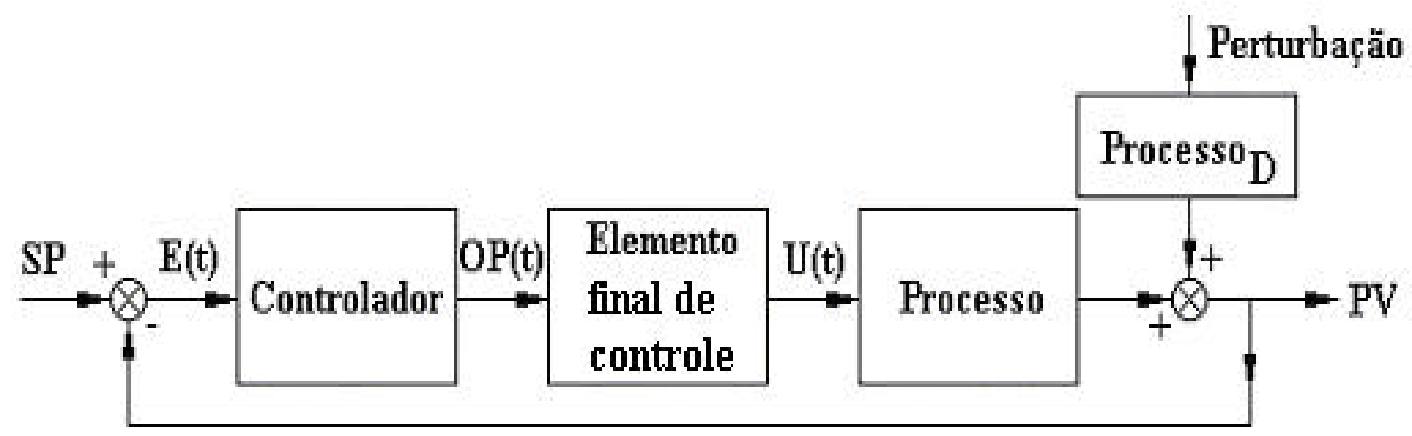

Figura 5.2 - Diagrama em blocos da malha fechada.

Nessa etapa a simulação dinâmica está quase concluída, restando apenas chavear a simulação do modo estático para o dinâmico. Esta operação é simples, e consiste do simulador resolver o problema correspondente ao estado estacionário do modo dinâmico utilizando os valores das variáveis independentes e as propriedades iniciais da simulação estática. Como o conjunto de equações é ligeiramente diferente do utilizado no modo estático, este fato faz com que tenhamos ligeiras diferenças entre os estados estacionários dos dois modos.

Estando no modo dinâmico podemos iniciar os testes para obtenção dos modelos do processo. 


\section{6}

\section{COMPORTAMENTO DO PROCESSO}

A coluna depropanizadora tem como restrições especificar o teor do componente chave pesado no propano, representado pelo $\mathrm{C}_{4}{ }^{+}$, e o componente chave leve do butano, representado pelo teor de $\mathrm{C}_{3}{ }^{-}$. O objetivo neste caso é limitar a quantidade de frações pesadas no propano para termos o mínimo de perda de butanos. Avaliaremos a variação destas especificações em toda a região de operação das variáveis manipuladas.

A característica básica do controle dual é a especificação dos produtos de topo e fundo da coluna, sendo que, no processo de separação a qualidade do produto de topo está ligada à do fundo através do fracionamento da coluna. $\mathrm{O}$ fracionamento é definido principalmente pela relação $\mathrm{L} / \mathrm{V}$, que é estabelecido externamente pela vazão de refluxo e a carga térmica do refervedor. A figura 6.1 apresenta a relação entre as qualidades dos dois produtos para diferentes valores de $\mathrm{Q}_{\mathrm{Reb}} \mathrm{e} \mathrm{L} / \mathrm{V}$. Vemos que, para um dado $\mathrm{Q}_{\mathrm{Reb}}$, a relação entre as duas saídas é razoavelmente linear em grande parte do diagrama. Isto indica que as duas saídas nessa região são lineares em relação a L/V. Entretanto, para valores de $\mathrm{C}_{3}^{-}$e $\mathrm{C}_{4}{ }^{+}$abaixo de $3 \%$, esse comportamento deixa de ser linear, e a nãolinearidade aumenta acentuadamente na região indicada na figura.

Quando a carga térmica do refervedor aumenta, estamos aumentando o fracionamento da coluna, que é representado pelas curvas mais a esquerda da figura.

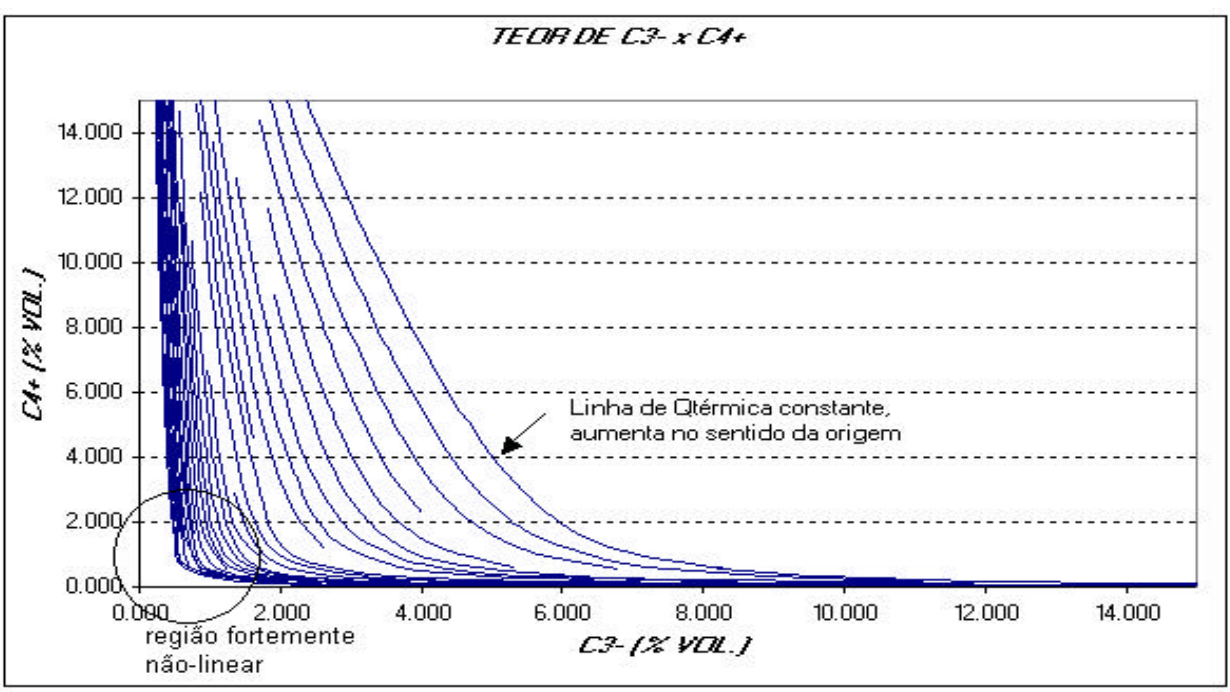

Figura 6.1 - Dualidade das qualidades dos produtos da coluna 
As variáveis manipuladas pelo controle supervisório terão especial atenção no estudo, pois afetam diretamente a qualidade dos produtos. No próximo item apresentamos o comportamento do processo para as duas variáveis de controle regulatório consideradas neste estudo.

\section{1 - Região de operação e ganhos do processo:}

A região de operação foi levantada considerando-se as seguintes variáveis no controle preditivo:

- Variáveis Controladas

$\checkmark$ Teor de $\mathrm{C}_{4}{ }^{+}$no propano $\left(\mathrm{C}_{4}{ }^{+}\right)[\%]$

$\checkmark$ Temperatura de topo (Ttopo) [kPa], esta é uma variável redundante em relação ao teor de $\mathrm{C}_{4}{ }^{+}$. Esta variável será utilizada em substituição ao teor de $\mathrm{C}_{4}{ }^{+}$, pois a inferência utilizada atualmente para cálculo deste teor não apresenta resultados satisfatórios.

$\checkmark$ Teor de $\mathrm{C}_{3}^{-}$no butano $\left(\mathrm{C}_{3}^{-}\right)[\%]$

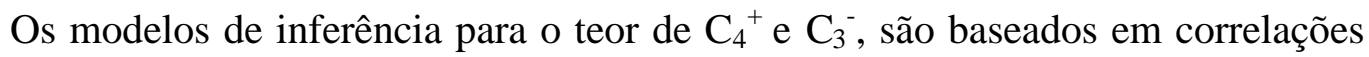
multilineares utilizando-se dados de análise cromatográfica das correntes de propano e butano e variáveis operacionais da planta industrial. Para obtenção das correlações, normalmente utiliza-se o método dos mínimos quadrados para ajuste dos parâmetros das correlações. Esta metodologia apesar de em alguns casos apresentar resultados satisfatórios a nível industrial, não é a mais recomendada principalmente nos casos em que os produtos têm pureza relativamente elevada como no caso da coluna depropanizadora. Assim, a utilização de outras metodologias deve ser testada principalmente aquelas que levem em conta modelos fundamentais do equilíbrio líquido-vapor. Os modelos utilizados atualmente para o teor de $\mathrm{C}_{4}^{+}$e $^{\mathrm{C}_{3}^{-}}$são apresentados a seguir:

$$
\begin{aligned}
& C_{3}^{-}=a * x_{1}+b^{*} x_{2} *\left(x_{3}-x_{4}\right) /\left(x_{5} * x_{6}\right)+c * x_{7}+d * x_{8}+e^{*} x_{9}+f \\
& C_{4}^{+}=a *\left(x_{1}\right)^{2}+b^{*} x_{2}+c^{*} x_{3}+d^{*} x_{4}+e
\end{aligned}
$$




\begin{tabular}{|c|c|c|c|}
\hline PARÂMETROS PARA & TEOR DE C ${ }_{3}^{-}$ & PARÂMETROS PARA & TEOR DE C $_{4}^{+}$ \\
\hline Coeficientes & Variáveis & Coeficientes & Variáveis \\
\hline$a=-0.6586$ & $\mathrm{x}_{1}=$ temp . fundo & $\mathrm{a}=0.3714$ & $\mathrm{x}_{1}=$ temp. topo \\
\hline $\mathrm{b}=-1.6912$ & $\mathrm{x}_{2}=$ vazão GOP & $\mathrm{b}=0.6198$ & $\mathrm{x}_{2}=$ pressão estágio 24 \\
\hline $\mathrm{c}=-0.0239$ & $\mathrm{x}_{3}=$ temp. GOP & $\mathrm{c}=-19.8385$ & $\mathrm{x}_{3}=$ temp. topo \\
\hline $\mathrm{d}=0.02027$ & $\mathrm{x}_{4}=$ temp. fundo & $\mathrm{d}=-0.04781$ & $\mathrm{x}_{4}=$ temp. refluxo \\
\hline $\mathrm{e}=0.1720$ & $\mathrm{x}_{5}=$ vazão carga & $\mathrm{e}=464.19$ & - \\
\hline$f=50.80$ & $\mathrm{x}_{6}=$ temp . carga & - & - \\
\hline- & $\mathrm{x}_{7}=$ temp. estágio 24 & - & - \\
\hline- & $\mathrm{x}_{8}=$ pressão estágio 24 & - & - \\
\hline - & $\begin{array}{l}\mathrm{x}_{9}=\text { temp. saída } \\
\text { refervedor }\end{array}$ & - & - \\
\hline
\end{tabular}

Tabela 6.1 - Parâmetros dos modelos das inferências.

- Variáveis manipuladas

$\checkmark$ Vazão de refluxo de topo $\left(\mathrm{V}_{\mathrm{RF}}\right)\left[\mathrm{m}^{3} / \mathrm{d}\right]$

$\checkmark$ Carga térmica do refervedor $\left(\mathrm{Q}_{\mathrm{REB}}\right)[\mathrm{MMcal} / \mathrm{h}]$, o controlador preditivo manipulará a vazão de fluido de aquecimento (gasóleo pesado - $\mathrm{V}_{\mathrm{GOP}}$ ) para o refervedor.

- Variáveis perturbadoras (são variáveis independentes e que são medidas)

$\checkmark$ Vazão de carga $\left(\mathrm{V}_{\mathrm{FD}}\right)\left[\mathrm{m}^{3} / \mathrm{d}\right]$

$\checkmark$ Temperatura do fluido de aquecimento $\left(\mathrm{T}_{\mathrm{GOP}}\right)\left[{ }^{\circ} \mathrm{C}\right]$

Como temos uma série de restrições a serem obedecidas, as variáveis manipuladas não poderão assumir valores quaisquer. A região delimitada por estas condições é chamada de região de operação do sistema. No nosso caso a região de operação pode ser definida pelas seguintes restrições:

$\checkmark$ Existe um estado estacionário, isto é, há líquido em todos os estágios da coluna;

$\checkmark$ Quantidade máxima de $\mathrm{C}_{4}{ }^{+}$no propano em função da perda de componentes nobres (iso-butano). A perda elevada de iso-butano na corrente de propano irá implicar na necessidade de importação deste componente para suprir as 
necessidades da unidade de gasolina de aviação. $O$ valor máximo considerado de butanos $\left(\mathrm{C}_{4}^{+}\right)$foi de $5 \%$.

$\checkmark$ Quantidade máxima de $\mathrm{C}_{3}{ }^{-}$no butano, a presença de componentes leves na corrente de butano irá implicar na perda de controle operacional na unidade de gasolina de aviação, ocasionando a queima de produtos nobres no sistema de tocha da refinaria. $\mathrm{O}$ valor máximo considerado de $\mathrm{C}_{3}^{-}$foi de $5 \%$.

O sistema apresenta folgas de projeto em relação às cargas térmicas do condensador e refervedor, não apresentando restrições quanto à vazões de refluxo ou de fluído de aquecimento para o refervedor. A hidráulica da coluna também apresenta folga, sendo possível processar todo o GLP produzido na unidade bem como correntes provenientes de outras unidades.

Diante das restrições acima, temos a região de operação mostrada na figura 6.2, onde vemos que há uma série limitada de pares de valores das variáveis manipuladas onde o processo é viável. Para levantamento da região de operação, as variáveis perturbadoras são mantidas constantes.

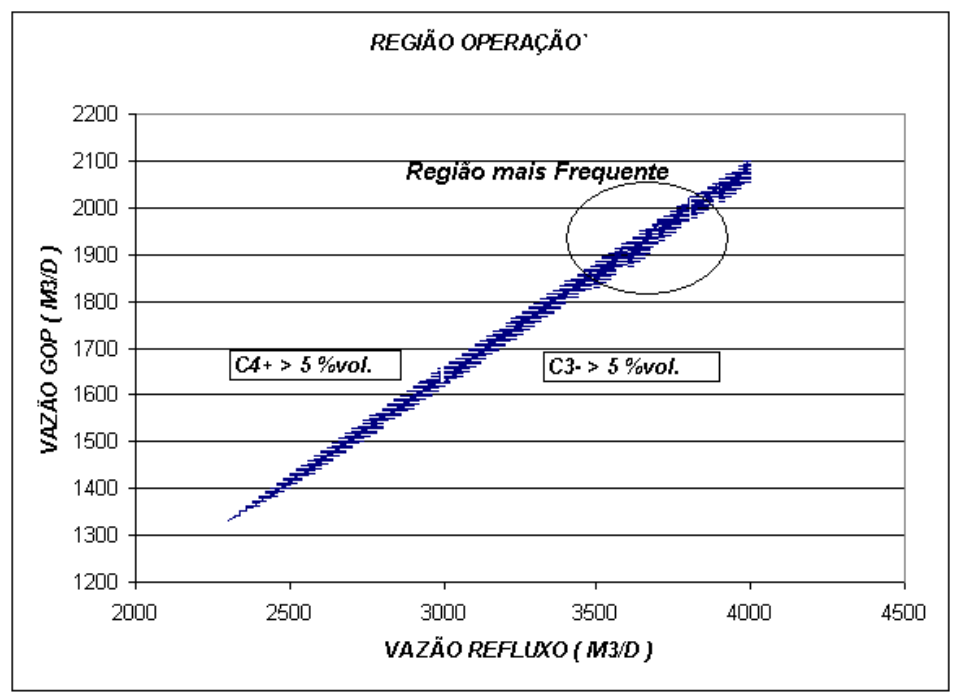

Figura 6.2 - Região de operação das variáveis manipuladas

Uma maneira eficiente para se observar o comportamento do processo é avaliar as curvas de ganhos do processo. Esses ganhos são estimados utilizando-se a derivada em vários pontos ao longo da região de operação, por exemplo para o teor de $\mathrm{C}_{3}{ }^{-} \mathrm{em}$ 
relação à vazão de refluxo teríamos:

$$
K p C_{3, i}^{-}=\left(C_{3, i}^{-}-C_{3, i-1}^{-}\right) /\left(V_{R F, i}-V_{R F, i-1}\right)
$$

sendo:

$K p C_{3, i}^{-}$- ganho estático para o teor de $\mathrm{C}_{3}{ }^{-}$no ponto de operação atual.

$C_{3, i}^{-}$- teor de $\mathrm{C}_{3}{ }^{-}$no ponto de operação atual.

$C_{3, i-1}^{-}$- teor de $\mathrm{C}_{3}{ }^{-}$no ponto de operação anterior.

$V_{R F, i}$ - vazão de refluxo no ponto de operação atual.

$V_{R F, i-1}$ - vazão de refluxo no ponto de operação anterior.

Estes ganhos estão representadas nas figuras 6.3 a 6.8. Nas figuras podemos observar que os ganhos do $\mathrm{C}_{3}{ }^{-}$com a $\mathrm{V}_{\mathrm{RF}}$ (vazão de refluxo) variam de 0.001 a 0.17 $\% \operatorname{vol} /\left(\mathrm{m}^{3} / \mathrm{d}\right)$, do $\mathrm{C}_{3}{ }^{-}$com a $\mathrm{V}_{\mathrm{GOP}}$ (vazão de GOP) variam de -0.25 a $-0.0015 \%$ vol/( $\left.\mathrm{m}^{3} / \mathrm{d}\right)$, do teor de $\mathrm{C}_{4}{ }^{+}$com $\mathrm{V}_{\mathrm{RF}}$ variam de -0.07 a $-0.0005 \% \mathrm{vol} /\left(\mathrm{m}^{3} / \mathrm{d}\right)$ e com a $\mathrm{V}_{\mathrm{GOP}}$ variam de 0.0010 a 0.150 , da temperatura de topo com a $V_{R F}$ variam de -0.015 a -0.0450 ${ }^{\circ} \mathrm{C} /\left(\mathrm{m}^{3} / \mathrm{d}\right)$ e com a $\mathrm{V}_{\mathrm{GOP}}$ variam de 0.030 a $0.13{ }^{\circ} \mathrm{C} /\left(\mathrm{m}^{3} / \mathrm{d}\right)$. Isto mostra que, para um dado par (manipulada e controlada), os ganhos do processo variam muito, o que leva a crer que a utilização de um único modelo para o controlador preditivo, poderá levar a uma performance não adequada do sistema.

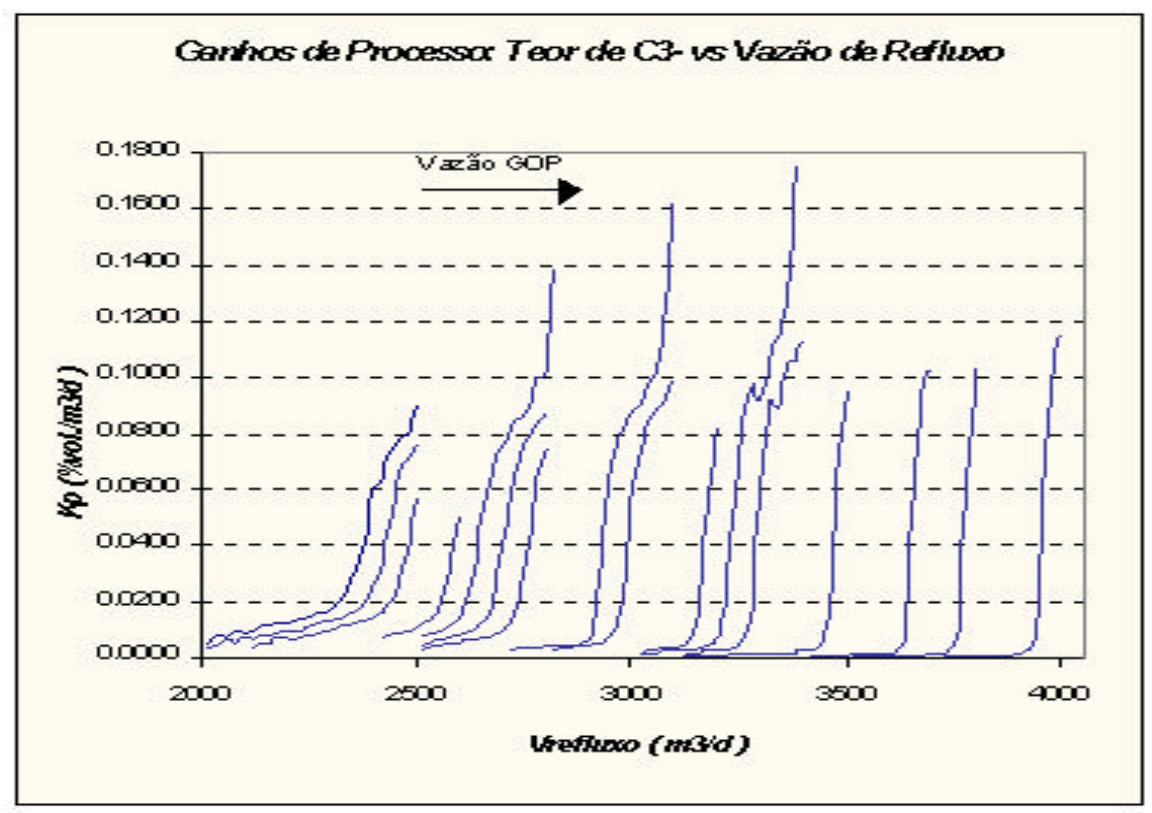

Figura 6.3 - Ganhos do C3 versus a vazão de refluxo 


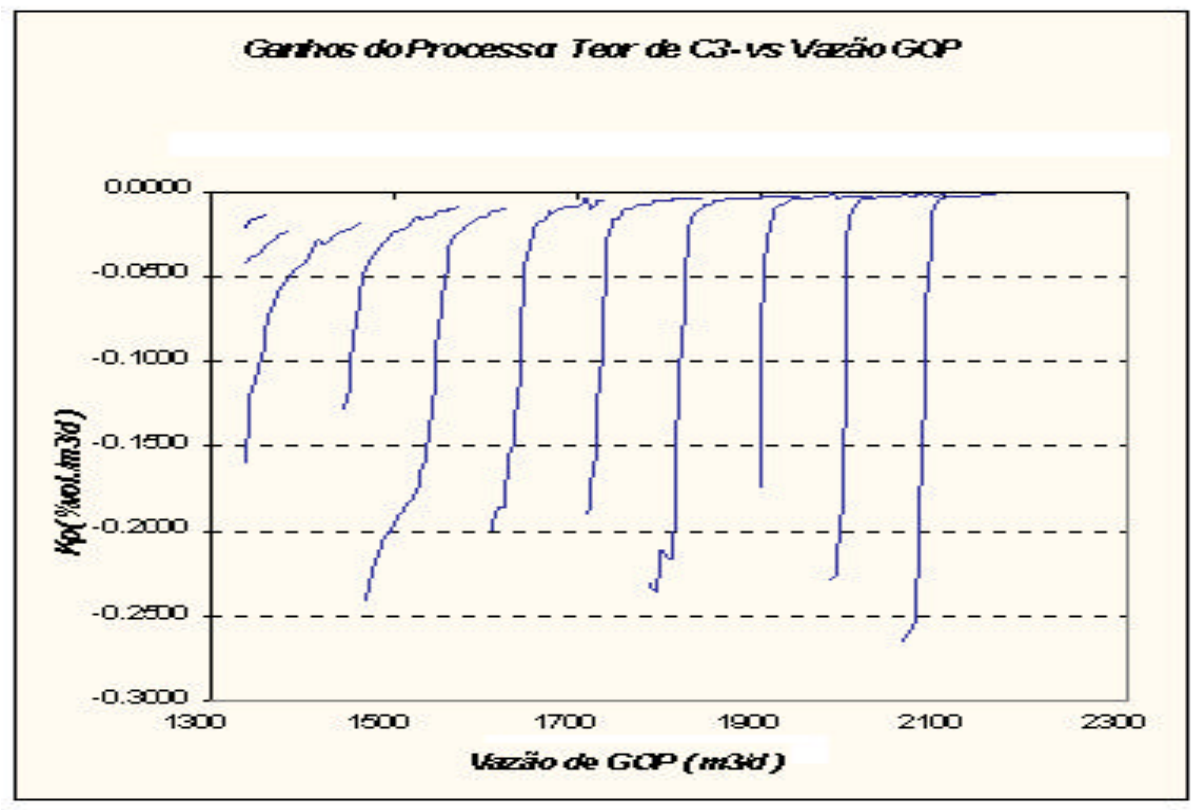

Figura 6.4 - Ganhos do C3- versus Vazão de GOP

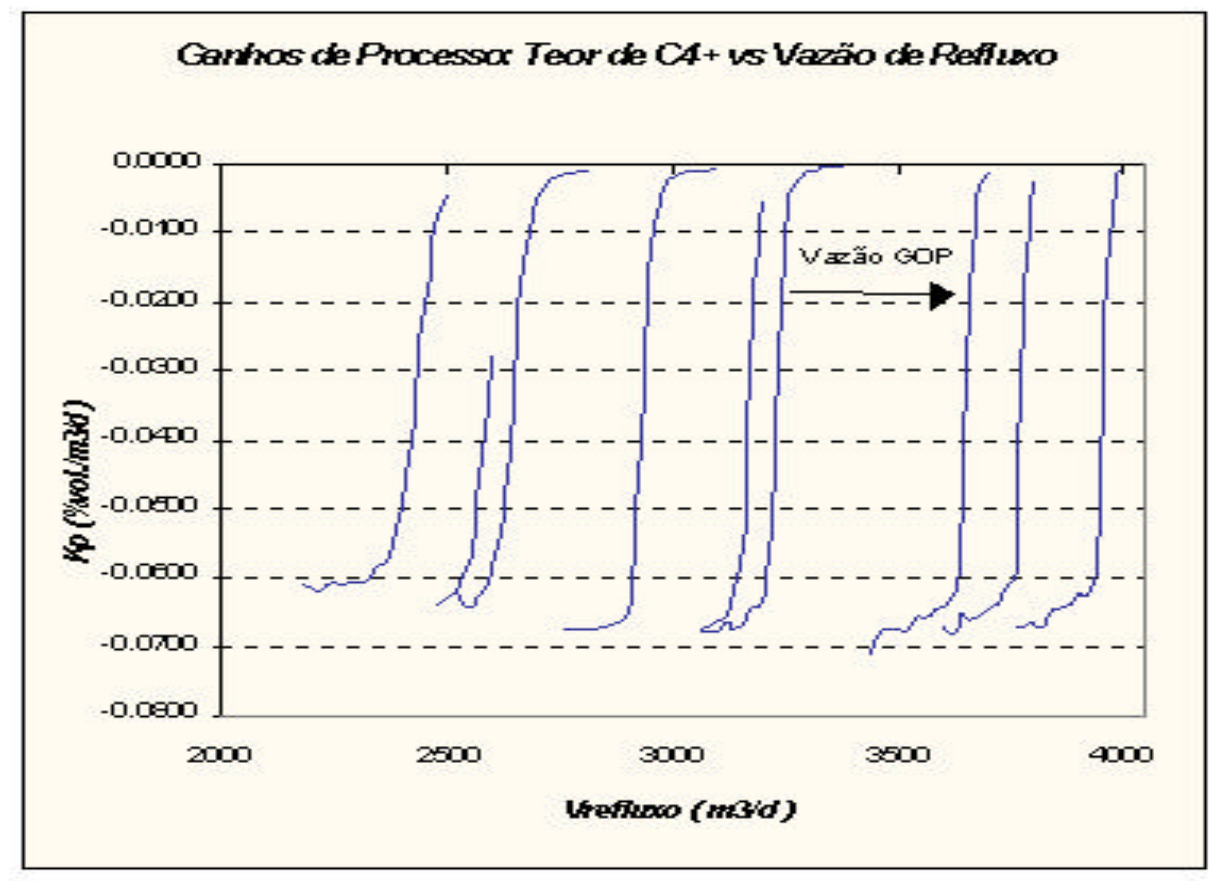

Figura 6.5 - Ganhos do $\mathrm{C}_{4}{ }^{+}$versus vazão de refluxo 


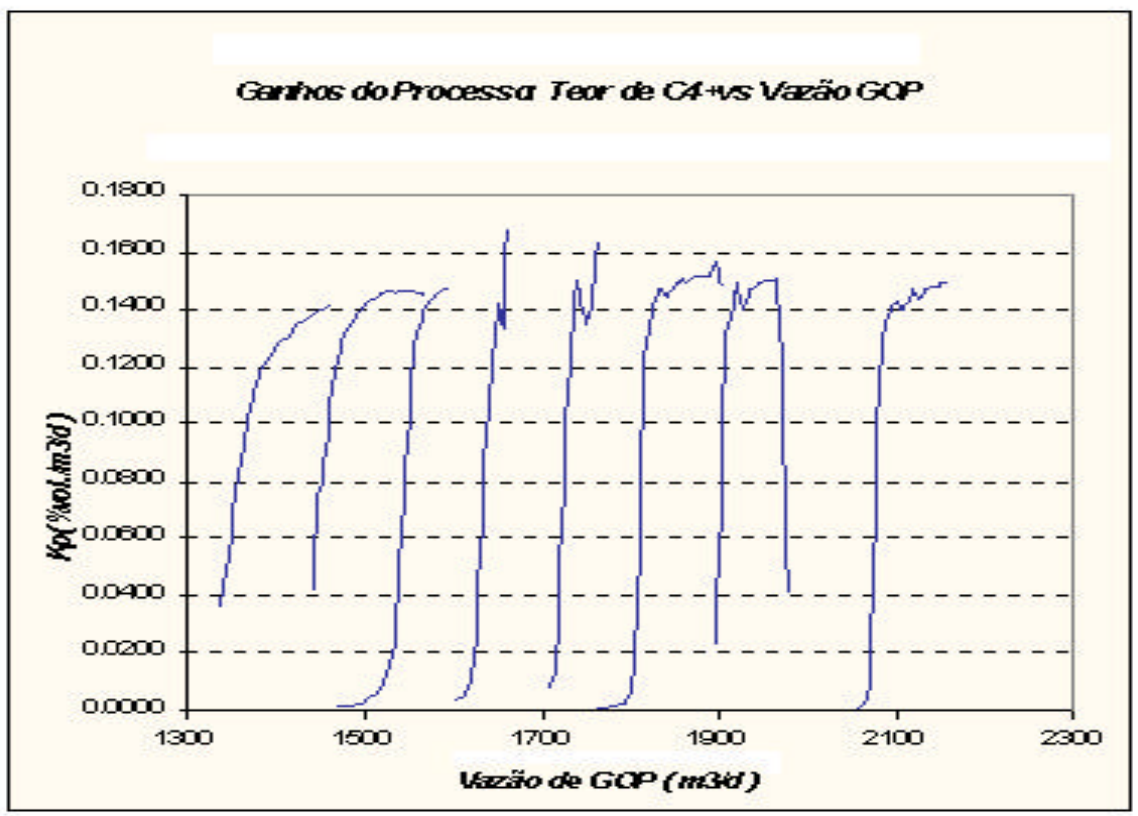

Figura 6.6 - Ganhos do $\mathrm{C}_{4}{ }^{+}$versus vazão de GOP

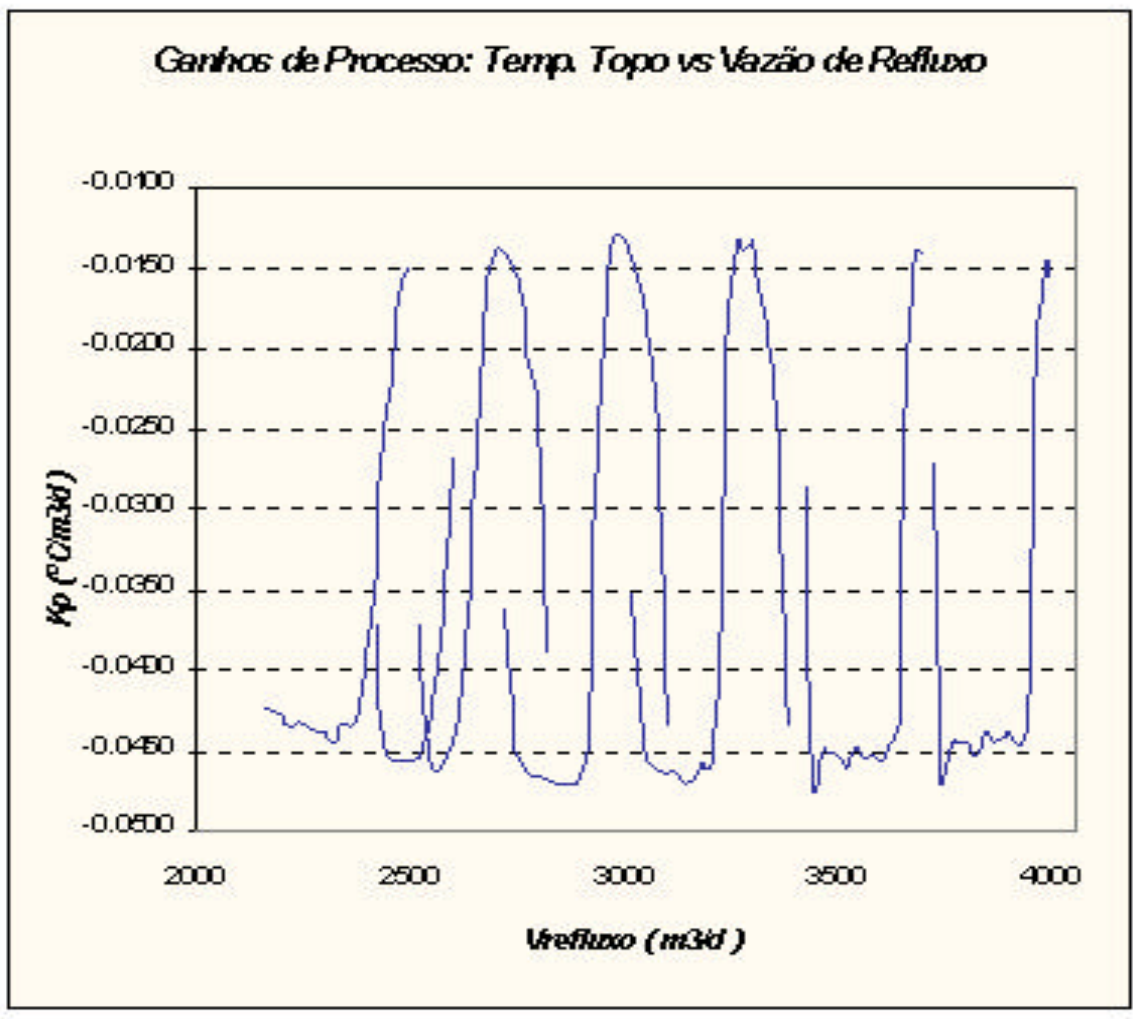

Figura 6.7 - Ganhos da temperatura de topo versus vazão de refluxo 


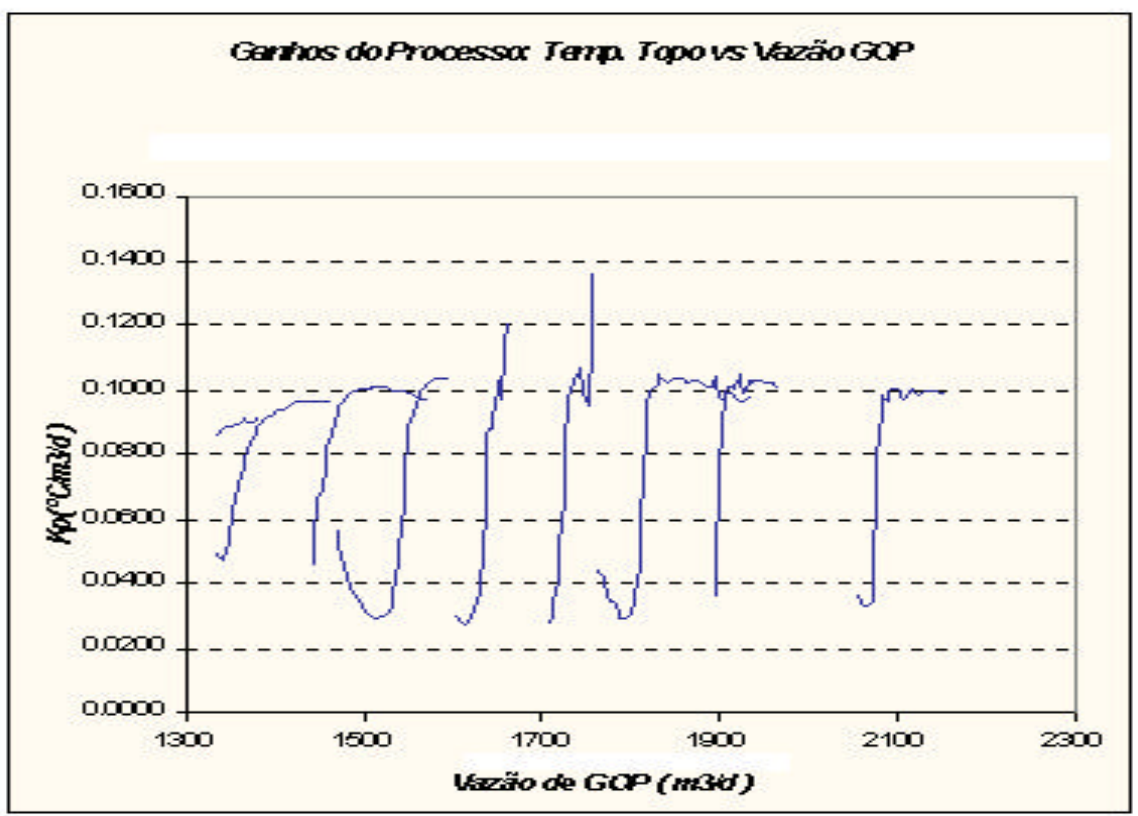

Figura 6.8 - Ganhos da temperatura de topo versus vazão de GOP

Diante dos valores apresentados, podemos concluir que o controle preditivo linear encontrará dificuldades para manter a qualidade dos produtos. Este fato é coerente com a observação do que ocorre em outras colunas de destilação nas plantas industriais, onde verifica-se que com esse tipo de variações no ganho do modelo o controlador tem reação muito lenta em determinadas condições e oscila excessivamente em outras condições de operação. Além disso, quando realizamos o teste em degrau na planta, em determinadas regiões de operação, as respostas das variáveis controladas são diferentes dependendo se aplicamos degraus positivos ou negativos e dependendo da amplitude do degrau. Isto é evidenciado nas tabelas 6.2 e 6.3, onde variamos o degrau de 1 a $5 \%$ nas variáveis manipuladas para cima e para baixo em torno do "caso base".

\begin{tabular}{|c|c|c|c|}
\hline Degrau (\%) & $\mathbf{K}_{\mathbf{p}} \mathbf{C}_{\mathbf{3}}^{-}$ & $\mathbf{K}_{\mathbf{p}} \mathbf{C}_{\mathbf{4}}{ }^{+}$ & $\mathbf{K}_{\mathbf{p}}$ Ttopo \\
\hline-5.0 & $8.44 \mathrm{E}-04$ & $-3.71 \mathrm{E}-02$ & $-2.43 \mathrm{E}-02$ \\
\hline-3.0 & $1.09 \mathrm{E}-03$ & $-3.90 \mathrm{E}-02$ & $-2.61 \mathrm{E}-02$ \\
\hline-1.0 & $2.04 \mathrm{E}-03$ & $-5.10 \mathrm{E}-02$ & $-3.58 \mathrm{E}-02$ \\
\hline & & & \\
\hline 1.0 & $5.54 \mathrm{E}-02$ & $-2.94 \mathrm{E}-02$ & $-2.75 \mathrm{E}-02$ \\
\hline 3.0 & $1.23 \mathrm{E}-01$ & $-1.02 \mathrm{E}-02$ & $-2.65 \mathrm{E}-02$ \\
\hline
\end{tabular}

Tabela 6.2 - Ganhos do processo em relação à vazão de refluxo. 


\begin{tabular}{|c|c|c|c|}
\hline Degrau (\%) & $\mathbf{K}_{\mathbf{p}} \mathbf{C}_{\mathbf{3}}^{-}$ & $\mathbf{K}_{\mathbf{p}} \mathbf{C}_{\mathbf{4}}{ }^{+}$ & $\mathbf{K}_{\mathbf{p}} \mathbf{T t o p o}$ \\
\hline-3.0 & $-6.49 \mathrm{E}-01$ & $2.10 \mathrm{E}-02$ & $9.60 \mathrm{E}-01$ \\
\hline-1.0 & $-1.36 \mathrm{E}-01$ & $5.79 \mathrm{E}-02$ & $5.70 \mathrm{E}-02$ \\
\hline & & & \\
\hline 1.0 & $-5.22 \mathrm{E}-03$ & $1.14 \mathrm{E}-01$ & $7.91 \mathrm{E}-02$ \\
\hline 3.0 & $-8.97 \mathrm{E}-03$ & $2.61 \mathrm{E}-01$ & $1.73 \mathrm{E}-01$ \\
\hline 5.0 & $-2.32 \mathrm{E}-03$ & $8.07 \mathrm{E}-02$ & $5.23 \mathrm{E}-02$ \\
\hline
\end{tabular}

Tabela 6.3 - Ganhos do processo para a vazão de GOP.

Nas tabelas 6.4 e 6.5 são apresentados os ganhos para as variáveis perturbadoras que serão utilizadas no controlador. Aqui também é possível observar a grande variação do ganho em função do degrau aplicado e do sentido (aumento ou redução da variável) do mesmo.

\begin{tabular}{|c|c|c|c|}
\hline Degrau (\%) & $\mathbf{K}_{\mathbf{p}} \mathbf{C}_{\mathbf{3}}^{-}$ & $\mathbf{K}_{\mathbf{p}} \mathbf{C}_{\mathbf{4}}{ }^{+}$ & $\mathbf{K}_{\mathbf{p}} \mathbf{T t o p o}$ \\
\hline-10.0 & 0.00192 & -0.01709 & -0.01216 \\
\hline-5.0 & 0.00155 & -0.01782 & -0.01255 \\
\hline-3.0 & 0.00128 & -0.01567 & -0.01089 \\
\hline-1.0 & 0.00096 & -0.01284 & -0.00859 \\
\hline & & & \\
\hline 1.0 & 0.00266 & -0.01525 & -0.01104 \\
\hline 3.0 & 0.00671 & -0.01350 & -0.01026 \\
\hline 5.0 & 0.01355 & -0.00923 & -0.00798 \\
\hline 10.0 & 0.02007 & -0.00478 & -0.00566 \\
\hline
\end{tabular}

Tabela 6.4 - Ganhos do processo para a vazão de carga .

\begin{tabular}{|c|c|c|c|}
\hline $\begin{array}{c}\text { Degrau } \\
\left({ }^{\mathbf{}} \mathbf{C}\right)\end{array}$ & $\mathbf{K}_{\mathbf{p}} \mathbf{C}_{\mathbf{3}}{ }^{-}$ & $\mathbf{K}_{\mathbf{p}} \mathbf{C}_{\mathbf{4}}{ }^{+}$ & $\mathbf{K}_{\mathbf{p}}$ Ttopo \\
\hline-3.6 & -3.42742 & 0.34303 & 0.73712 \\
\hline-1.6 & -2.09024 & 0.75228 & 0.78103 \\
\hline-0.6 & -0.48027 & 1.58481 & 1.18692 \\
\hline & & & \\
\hline 0.6 & -0.11253 & 2.00225 & 1.42429 \\
\hline 1.6 & -0.05985 & 1.39626 & 0.95874 \\
\hline 3.6 & -0.04473 & 1.22596 & 0.82054 \\
\hline
\end{tabular}

Tabela 6.5 - Ganhos do processo para a temperatura do fluido de aquecimento. 


\section{7 \\ IDENTIFICAÇÃO DOS MODELOS DO SISTEMA}

Podemos dizer que a identificação do modelo do processo é uma das etapas mais importantes no projeto e análise de um controlador, pois caso se utilize um modelo inadequado, a performance do controle ficará prejudicada e sua análise será incorreta. Assim, devemos ter em mente uma série de considerações sobre os modelos a serem utilizados, tais como:

- Que tipo de modelo o controlador utiliza?

Para responder esta pergunta devemos considerar uma série de características do sistema, como: se o modelo deve ser linear ou não-linear, fenomenológico ou empírico, invariante ou variante no tempo.

- Qual a forma de apresentação do modelo?

Devemos responder se o modelo deve ser apresentado na forma de função de transferência, ou resposta ao degrau ou impulso. Se apresentarmos o modelo na forma de função de transferência, devemos definir se o modelo será apresentado no domínio de tempo contínuo ou discreto. Se o modelo estiver na forma de resposta ao degrau ou impulso, podemos apresentá-lo na forma de série temporal ou na forma analítica.

- Qual a necessidade de se utilizar mais de um modelo do processo?

Para sistemas variantes no tempo, muitas vezes um único modelo não atende à performance desejada em toda a região de operação do sistema. Isto ocorre quando o processo altera suas características, por exemplo, alteração da natureza da matéria-prima, ou degradação do processo por desgaste do equipamento, como colunas de destilação cuja eficiência do estágio cai por vazamento nas bandejas. Neste caso, devemos decidir se iremos utilizar um conjunto de modelos pré-definidos ou se adaptaremos algum parâmetro do modelo do processo, por exemplo o seu ganho estático.

No caso da coluna depropanizadora temos regiões de fortes não-linearidades nos ganhos, que devem ter, portanto, grandes alterações na sua dinâmica, e temos regiões 
onde os modelos obtidos são incompatíveis sob o aspecto de utilização em controle preditivo.

\section{1 - Algoritmo de identificação com modelos na forma analítica}

Neste trabalho utilizaremos um conjunto de modelos convenientemente escolhidos sendo apresentados na forma de resposta ao degrau analítico no domínio do tempo contínuo. Esta representação pode ser visualizada através das seguintes equações:

$$
\begin{aligned}
& s_{i, j, n}=0 \forall n T \leq \theta_{i, j} \\
& s_{i, j, n}=c_{i, j, 0}+\sum_{g=1}^{n a} c_{i, j, g} f_{i, j, g}(n T) \quad \forall n T>\theta_{i, j}
\end{aligned}
$$

sendo:

$$
\begin{array}{ll}
i & \text { - i-ésima variável de saída (controlada) } \\
j & \text { - j-ésima variável de entrada (manipulada e perturbação) } \\
\theta_{i, j} \quad \text { - tempo morto do par de variáveis } \mathrm{i}, \mathrm{j} \\
n \quad \text { - instante de amostragem } \\
f_{i, j, 0}(t)=1 \\
f_{i, j, g}(t)=e^{r_{i, j, g}\left(t-\theta_{i, j}\right)} \quad \forall r_{i, j, g} \neq 0 \\
f_{i, j, g}(t)=t-\theta_{i, j} \quad \forall r_{i, j, g}=0
\end{array}
$$

O comportamento dinâmico da maioria dos processos pode ser representado por equações diferenciais de primeira ou segunda ordem. No caso de processos em série, como por exemplo "n” estágios de equilíbrio, a função de transferência resultante tem a ordem correspondente à multiplicação das várias funções de transferência. Felizmente os processos químicos de ordens elevadas têm normalmente comportamento que pode ser razoavelmente aproximado por um modelo de ordem menor. Os controladores em malha fechada podem adicionar raízes complexas ou zeros às funções de transferências. Além disso, sistemas com funções de transferência em paralelo, podem adicionar zeros no processo resultante, gerando por exemplo, resposta inversa.

Diante deste comportamento, podemos dizer que a maioria dos processos podem ser representados pela seguinte função de transferência: 


$$
G(s)=\frac{b_{0}+b_{1} s}{1+a_{1} s+a_{2} s^{2}} e^{-s \theta}
$$

Considerando o modelo sem a componente do tempo morto, temos:

$$
G(s)=\frac{b_{0}+b_{1} s}{1+a_{1} s+a_{2} s^{2}}
$$

Considerando a aplicação de um degrau unitário ao sistema temos:

$$
y_{d}(s)=G(s) \times \frac{1}{s}
$$

substituindo a equação (7.3) em (7.4), temos:

$$
y_{d}(s)=\frac{b_{0}+b_{1} s}{1+a_{1} s+a_{2} s^{2}} \times \frac{1}{s}
$$

fatorando $y_{d}(s)$, temos:

$$
y_{d}(s)=\frac{b_{0}+b_{1} s}{\left(\tau_{1} s+1\right)\left(\tau_{2} s+1\right) s}
$$

ou

$$
y_{d}(s)=\frac{1}{\tau_{1} \tau_{2}} \frac{b_{0}+b_{1} s}{\left(s+\frac{1}{\tau_{1}}\right)\left(s+\frac{1}{\tau_{2}}\right) s}
$$

Portanto as raízes do denominador são:

$$
r_{1}=-\frac{1}{\tau_{1}} \quad ; \quad r_{2}=-\frac{1}{\tau_{2}} \quad ; \quad r_{3}=0
$$

Então a resposta ao degrau do sistema pode ser representada por:

$$
y_{d}(s)=r_{1} r_{2} \frac{b_{0}+b_{1} s}{\left(s-r_{1}\right)\left(s-r_{2}\right) s}
$$

Separando a resposta ao degrau em frações parciais, temos: 


$$
y_{d}(s)=\frac{c_{0}}{s}+\frac{c_{1}}{s-r_{1}}+\frac{c_{2}}{s-r_{2}}
$$

sendo:

$$
\begin{aligned}
& c_{0}=b_{0} \\
& c_{1}=r_{2} \frac{b_{0}+b_{1} r_{1}}{r_{1}-r_{2}} \text { ou } c_{1}=\frac{b_{0} \frac{1}{r_{1}}+b_{1}}{\frac{1}{r_{2}}-\frac{1}{r_{1}}} \\
& c_{2}=r_{1} \frac{b_{0}+b_{1} r_{2}}{r_{2}-r_{1}} \quad \text { ou } \quad c_{2}=\frac{b_{0} \frac{1}{r_{2}}+b_{1}}{\frac{1}{r_{1}}-\frac{1}{r_{2}}}
\end{aligned}
$$

Passando a resposta ao degrau para o domínio do tempo e incluindo o termo do tempo morto, teremos:

$$
y_{d}(t)=c_{0}+c_{1} e^{r_{1}(t-\theta)}+c_{2} e^{r_{2}(t-\theta)}
$$

No caso de um sistema SISO, podemos escrever a equação da predição da saída para um instante qualquer, na seguinte forma paramétrica:

$$
\begin{aligned}
& y_{j}=\left[p_{0}\right]_{k}+\sum_{g=1}^{n a}\left[p_{g}\right]_{k} f_{g}(j T-\theta) \\
& {\left[p_{0}\right]_{k}=\left[p_{0}\right]_{k-1}+c_{0} \Delta u_{k-1}} \\
& {\left[p_{g}\right]_{k}=\left[p_{g}\right]_{k-1} e^{r_{g} T}+c_{g} e^{r_{g} T} \Delta u_{k-1}}
\end{aligned}
$$

sendo:

$$
\begin{aligned}
& g=1,2, \ldots, n a \\
& j=\operatorname{int}\left(k+\frac{\theta}{T}+1\right)
\end{aligned}
$$

sendo que:

$$
\begin{array}{ll}
k=1,2, \ldots, n_{s}-\theta / T-1 \\
\theta & \text { - tempo morto } \\
T & \text { - período de amostragem } \\
n_{s} & \text { - horizonte da predição } \\
\operatorname{int}() & \text { - valor inteiro }
\end{array}
$$


na - número de pólos do processo

A identificação dos modelos do processo consiste na determinação dos parâmetros $c_{g}, r_{g}$ e $\theta$ para $g=0,1,2, \ldots, n a$ da equação (7.6). Este ajuste de parâmetros é efetuado através de um procedimento de busca não-linear. Normalmente na identificação do processo, os modelos são apresentados na forma discreta. Neste trabalho, os modelos serão apresentados na forma contínua.

A identificação do processo consiste das seguintes etapas:

1 - Assumir os valores iniciais para os parâmetros:

Considerando o modelo definido pela equação (7.2), a predição da saída que é dada pela equação (7.6), fica:

$$
y_{j}=\left[p_{0}\right]_{k}+\left[p_{1}\right]_{k} e^{r_{1}(j T-\theta)}+\left[p_{2}\right]_{k} e^{r_{2}(j T-\theta)}
$$

sendo:

$$
\begin{aligned}
& {\left[p_{1}\right]_{k}=\left[p_{1}\right]_{k-1} e^{r_{1} T}+c_{1} e^{r_{1} T} \Delta u_{k-1}} \\
& {\left[p_{2}\right]_{k}=\left[p_{2}\right]_{k-1} e^{r_{2} T}+c_{2} e^{r_{2} T} \Delta u_{k-1}}
\end{aligned}
$$

Os parâmetros a serem ajustados, para os quais devemos assumir valores iniciais, são: $c_{1}, c_{2}, r_{1}, r_{2}$ e $\theta$. Além disso, inicialmente assumimos que o processo esteja no estado estacionário, portanto:

$$
\begin{aligned}
& {\left[p_{0}\right]_{1}=y_{1}} \\
& {\left[p_{1}\right]_{1}=0} \\
& {\left[p_{2}\right]_{1}=0}
\end{aligned}
$$

sendo:

$y_{1}-$ Valor inicial da saída

2 - Calcular as predições da saída:

Nesta etapa calculamos as predições da saída nos instantes $k=1,2, \ldots, n_{s}-\theta / T-1$ com os parâmetros calculados através das equações (7.7) a (7.9).

3 - Calcular o resíduo da predição através do critério quadrático: 


$$
I S E=\sum_{j=\theta / T^{+1}}^{n_{s}} \gamma_{j}\left(\hat{y}_{j}-y_{j}\right)^{2}
$$

sendo:

$$
\begin{aligned}
& y_{j} \text { - valor medido da saída no instante } \mathrm{j} \\
& \hat{y}_{j} \text { - valor da predição da saída no instante } \mathrm{j} \\
& \gamma_{j} \text { - peso que pune o desvio no instante } \mathrm{j}
\end{aligned}
$$

4 - Executar o procedimento de otimização dos parâmetros do modelo, formulando o problema da seguinte forma:

$$
\min _{c_{1}, c_{2}, r_{1}, r_{2}, \theta} I S E
$$

sujeito à:

$$
\sum_{g=1}^{n a} c_{g}=-c_{0}
$$

A restrição apresentada pela equação (7.10) garante que o sistema seja estritamente próprio. Os parâmetros $c_{g}$ e $r_{g}$ do modelo podem ser complexos e o parâmetro $c_{0}$ é um número real. No caso do modelo utilizado neste trabalho, a equação (7.10) fica:

$$
-c_{0}=c_{1}+c_{2}
$$

Como a predição da saída é uma função exponencial, devemos utilizar um algoritmo da programação não-linear.

No processo de identificação foram efetuados testes que consistiram na aplicação de degraus nas variáveis manipuladas e perturbadoras individualmente, ou seja, aplicouse um degrau em uma dada entrada, aguardou-se o processo atingir o estado estacionário e em seguida se aplicou um degrau na variável seguinte. A escolha dos pontos de operação onde os testes foram executados levou em conta as faixas de ganhos do processo e a região de operação em que o controlador deverá operar.

No próximo item apresentamos os resultados da identificação dos modelos do processo para as duas composições de carga utilizadas. 


\section{2 - Modelos de processo identificados:}

Foram aplicados degraus nas seguintes variáveis manipuladas e perturbadoras do controle supervisório:

$\checkmark$ Vazão de refluxo de topo

$\checkmark$ Vazão de fluido de aquecimento

$\checkmark$ Vazão de carga

$\checkmark$ Temperatura do fluido de aquecimento $\left(\mathrm{T}_{\mathrm{GOP}}\right)$

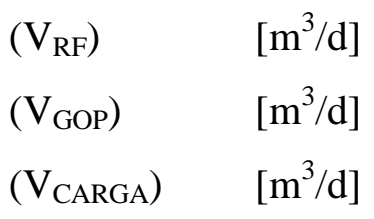

$\left[{ }^{\circ} \mathrm{C}\right]$

Foram efetuados testes em 5 pontos da região de operação do sistema. Na figura 7.1, vemos os pontos escolhidos assinalados de 1 a 5 , que correspondem aos números dos modelos identificados. Os modelos de 1 a 3 são referentes à composição de carga 1 e os modelos 4 e 5 se referem à composição de carga 2. O ponto FD corresponde a um modelo obtido através de teste realizado na planta industrial, o teste consistiu na aplicação de degraus nas variáveis manipuladas e perturbadoras, sendo o modelo obtido também na forma analítica como os demais.

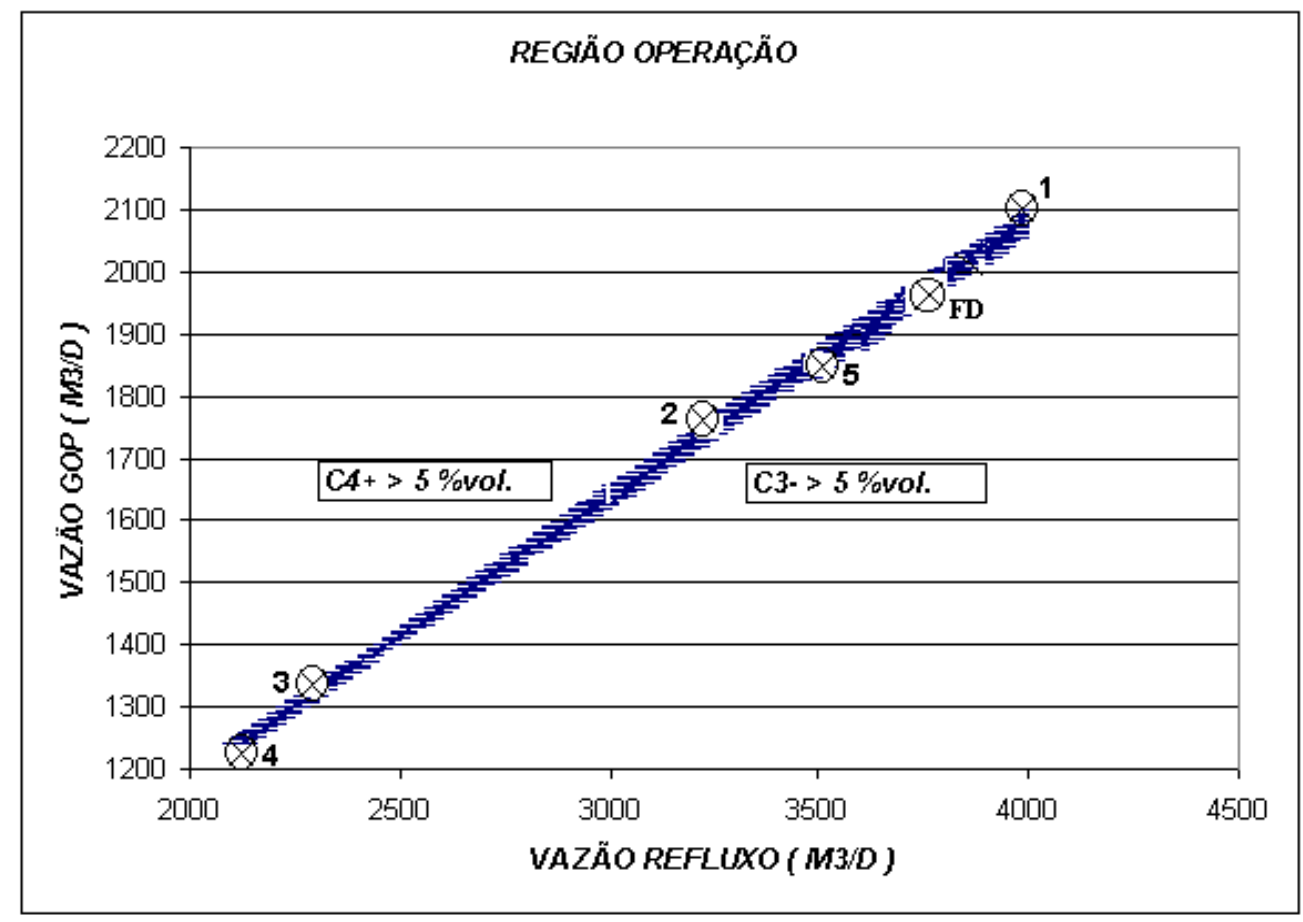

Figura 7.1 - Região de operação do processo. 
Os pontos foram escolhidos visando obter modelos que representem as diversas condições do processo de forma a termos um politopo onde os vértices seriam representados através deste conjunto de modelos. A idéia é então representar qualquer modelo correspondente à outra condição operacional através de uma combinação convexa deste conjunto de modelos.

Os modelos obtidos estão representando bem a região de operação do controlador. Na tabela 7.1 apresentamos os pontos de operação com suas principais características.

\begin{tabular}{|c|c|c|c|c|c|}
\hline Modelo & $\begin{array}{c}\mathbf{V}_{\mathbf{R F}} \\
\left(\mathbf{m}^{\mathbf{3}} / \mathbf{d}\right)\end{array}$ & $\begin{array}{c}\mathbf{V}_{\mathbf{G O P}} \\
\left(\mathbf{m}^{\mathbf{3}} / \mathbf{d}\right)\end{array}$ & $\begin{array}{c}\mathbf{T t o p o} \\
\left.\mathbf{(}^{\mathbf{0}} \mathbf{C}\right)\end{array}$ & $\begin{array}{c}\mathbf{C}_{\mathbf{4}}{ }^{+} \\
(\boldsymbol{\%})\end{array}$ & $\begin{array}{c}\mathbf{C}_{\mathbf{3}}{ }^{-} \\
(\boldsymbol{\%})\end{array}$ \\
\hline 1 & 4000 & 2104 & 47.74 & 4.30 & 0.38 \\
\hline 2 & 3160 & 1731 & 49.34 & 6.55 & 0.88 \\
\hline 3 & 2300 & 1331 & 47.82 & 4.60 & 4.81 \\
\hline 4 & 2240 & 1115 & 47.08 & 3.00 & 3.01 \\
\hline 5 & 3532 & 1837 & 44.35 & 0.08 & 4.35 \\
\hline
\end{tabular}

Tabela 7.1 - Pontos de operação dos modelos do processo

A figura 7.2 mostra o conjunto de modelos para resposta às variáveis manipuladas e a 7.3 mostra o conjunto de modelos para as perturbações. Observa-se que há modelos de naturezas muito distintas, com ganhos e dinâmicas bem diferentes. Nas figuras, não apresentamos os modelos relativos do teor $\boldsymbol{C}_{4}{ }^{+}$para a planta industrial, devido à não confiabilidade da inferência utilizada para medição desta variável. 


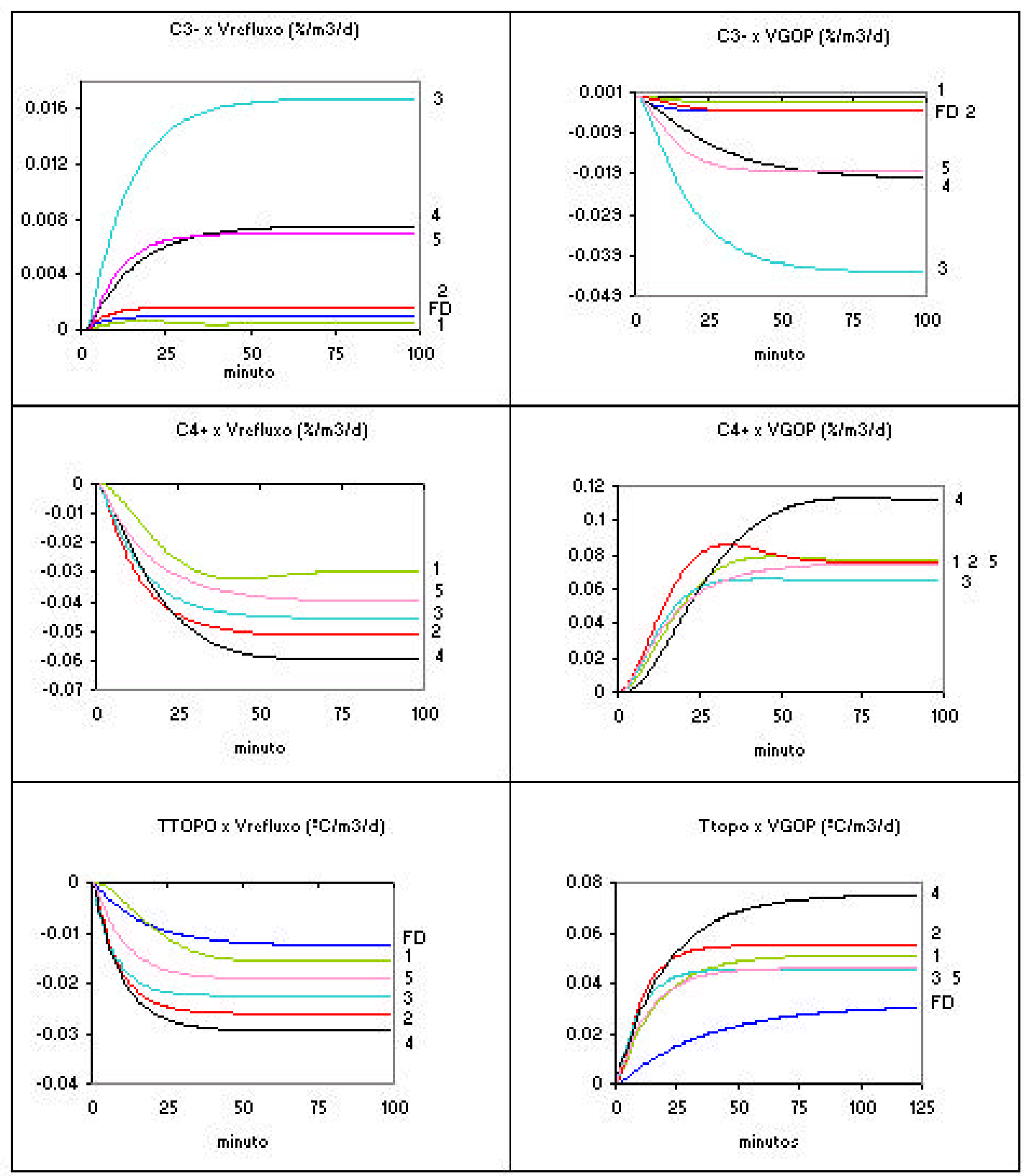

Figura 7.2 - Modelos de resposta ao degrau nas manipuladas. O modelo da planta real é o FD. 


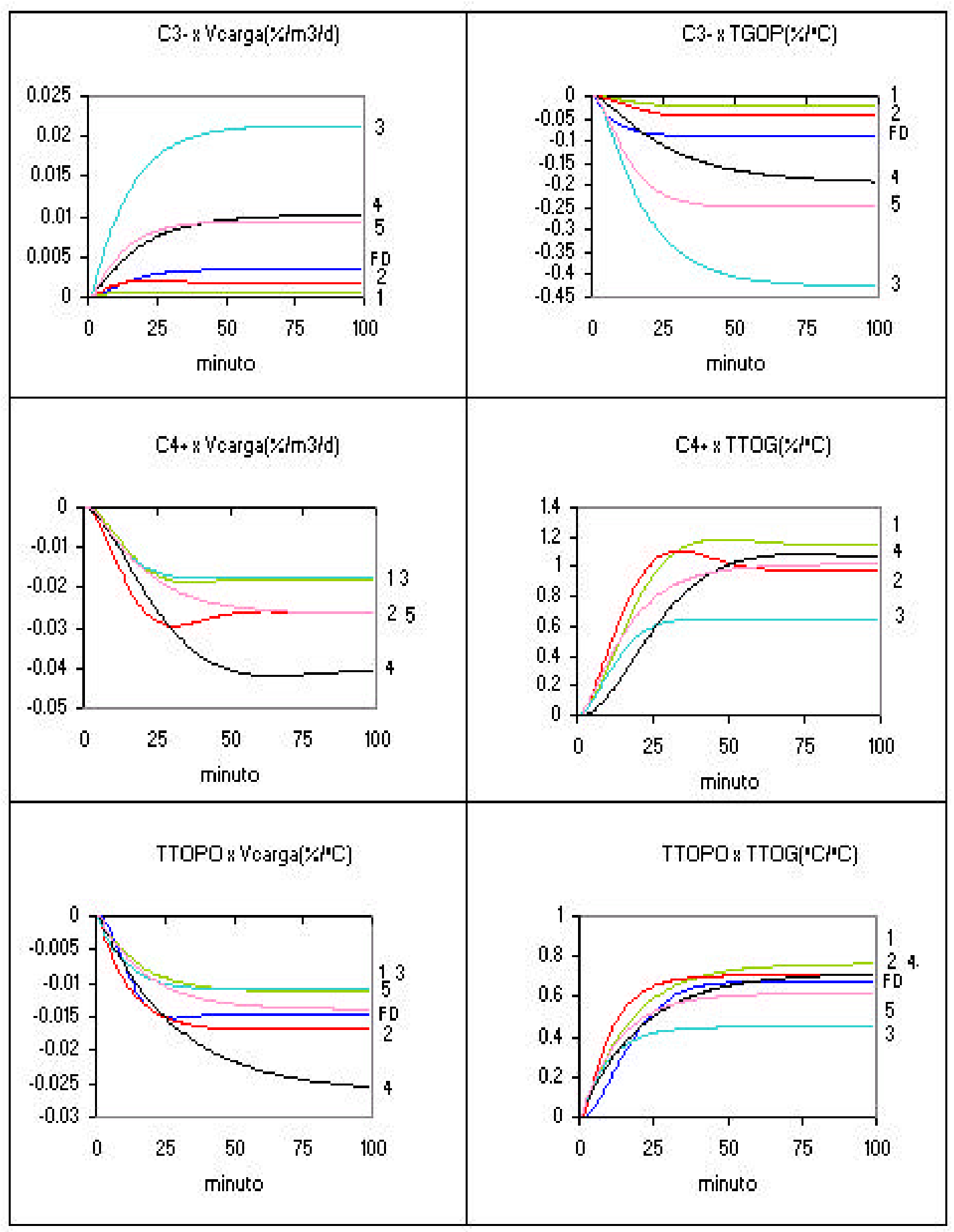

Figura 7.3 - Modelos de resposta ao degrau nas perturbações. O modelo da planta real é o FD. 
A seguir apresentamos os parâmetros dos modelos identificados, na forma de resposta ao degrau unitário analítica, conforme apresentado anteriormente. No Apêndice A, apresentamos os modelos na forma de função de transferência

\section{Para o modelo 1:}

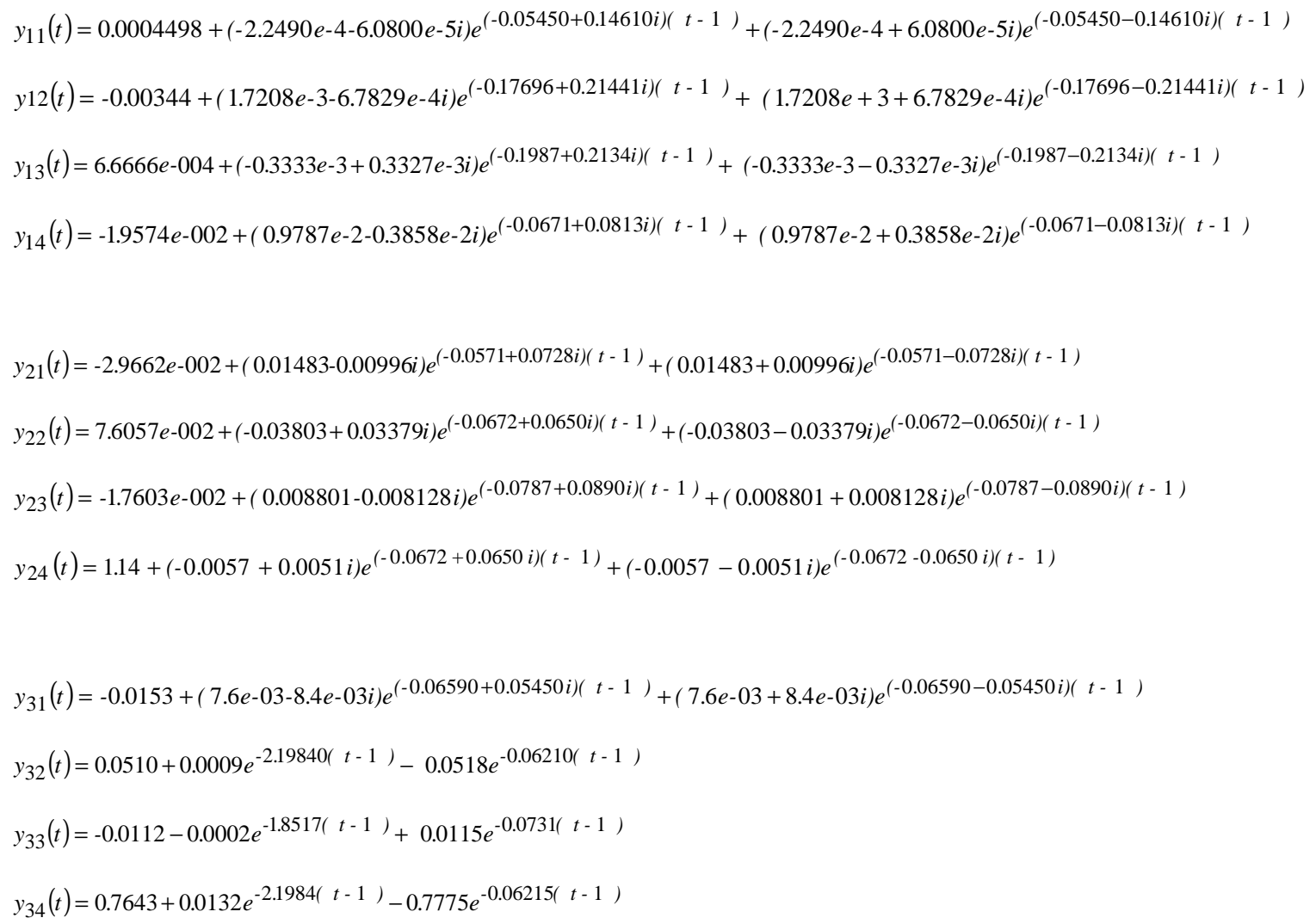

\section{Para o modelo 2:}

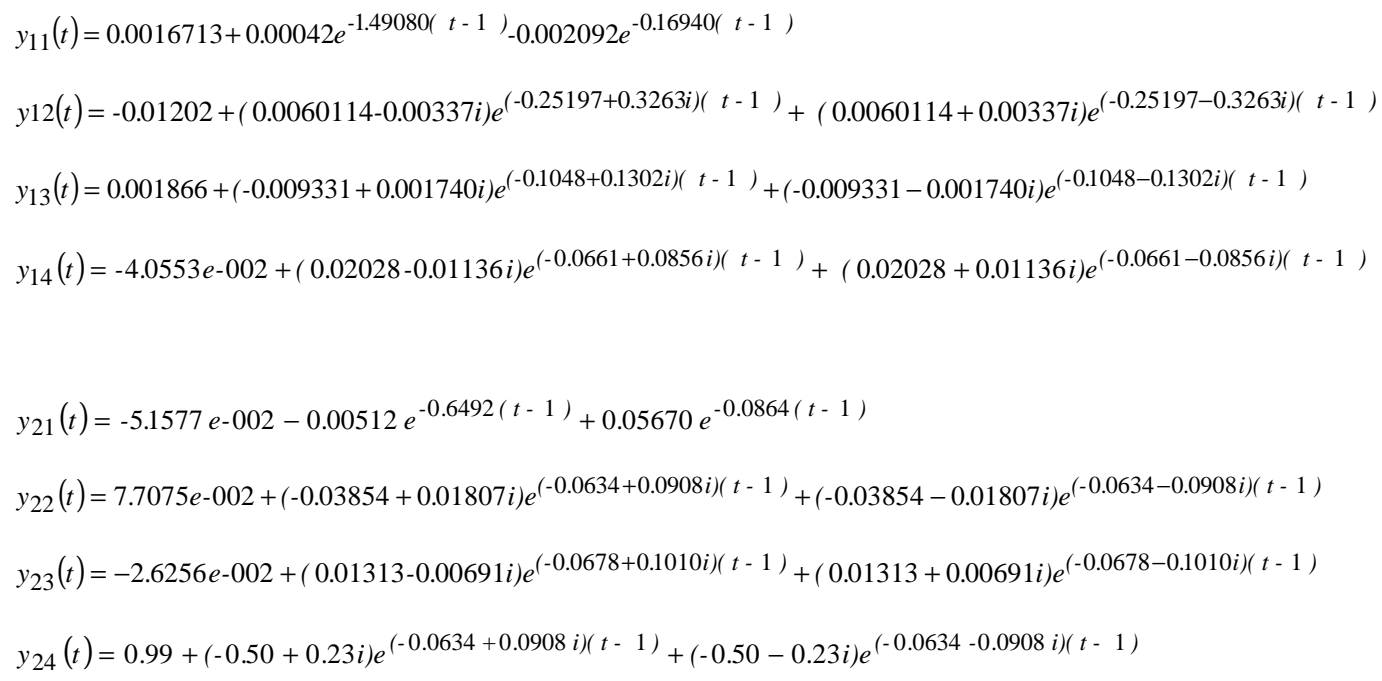




$$
\begin{aligned}
& y_{31}(t)=-0.0261+0.0001 e^{-1.94770(t-1)}+0.026 e^{-0.12470(t-1)} \\
& y_{32}(t)=0.055+0.0099 e^{-0.72290(t-1)}-0.0649 e^{-0.11240(t-1)} \\
& y_{33}(t)=-0.0170-0.0006 e^{-0.7390(t-1)}+0.0177 e^{-0.0938(t-1)} \\
& y_{34}(t)=0.7074+0.1269 e^{-0.7229(t-1)}-0.8343 e^{-0.1124(t-1)}
\end{aligned}
$$

\section{Para o modelo 3:}

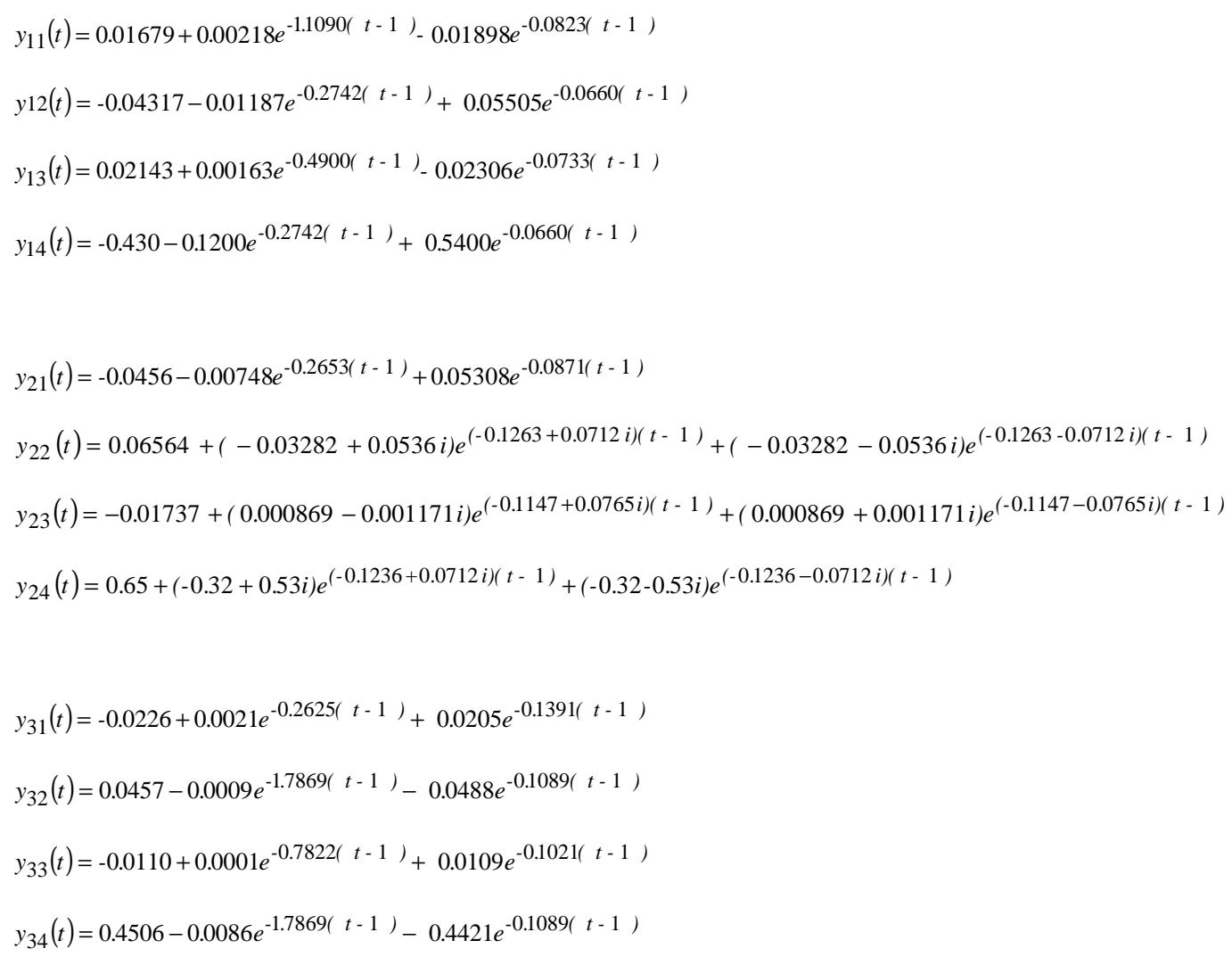

\section{Para o modelo 4:}

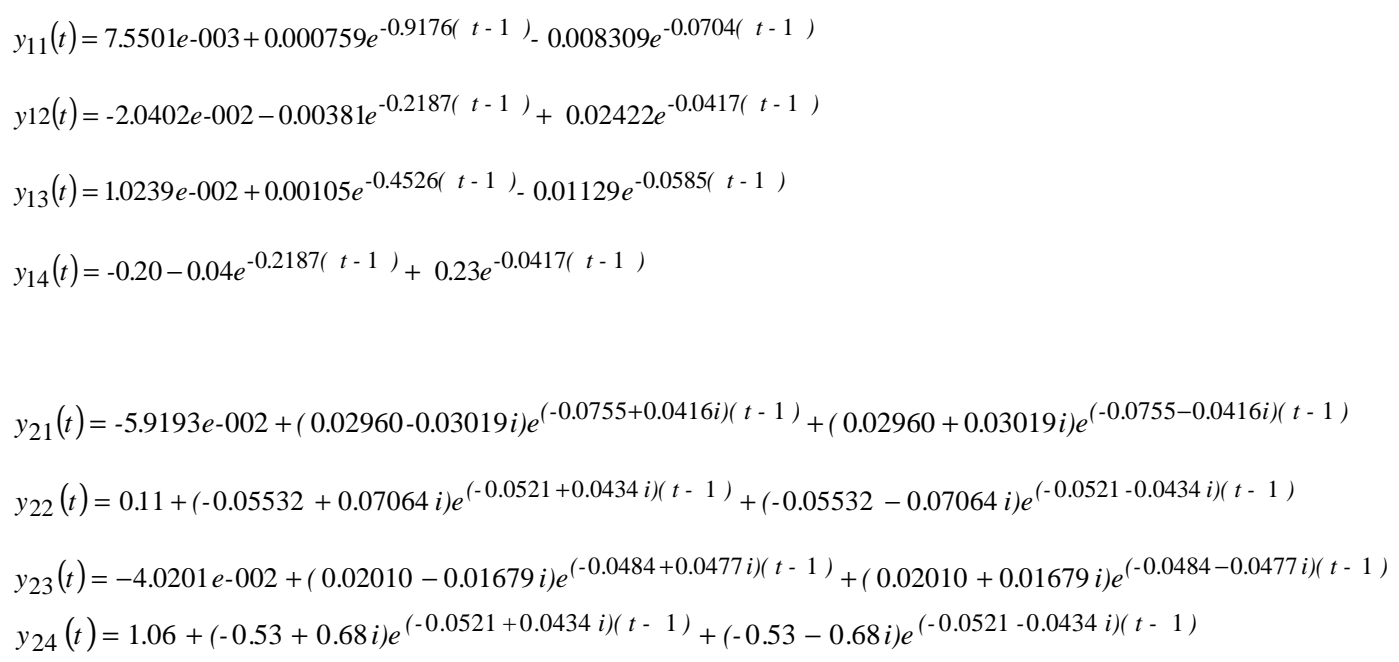




$$
\begin{aligned}
& y_{31}(t)=-0.0295+0.0010 e^{-0.7790(t-1)}+0.0285 e^{-0.1078(t-1)} \\
& y_{32}(t)=0.0751-0.0025 e^{-0.8120(t-1)}-0.0726 e^{-0.0482(t-1)} \\
& y_{33}(t)=-0.0263-0.0003 e^{-1.8516(t-1)}+0.0266 e^{-0.0361(t-1)} \\
& y_{34}(t)=0.7180-0.0237 e^{-0.8120(t-1)}-0.6943 e^{-0.0482(t-1)}
\end{aligned}
$$

\section{Para o modelo 5:}

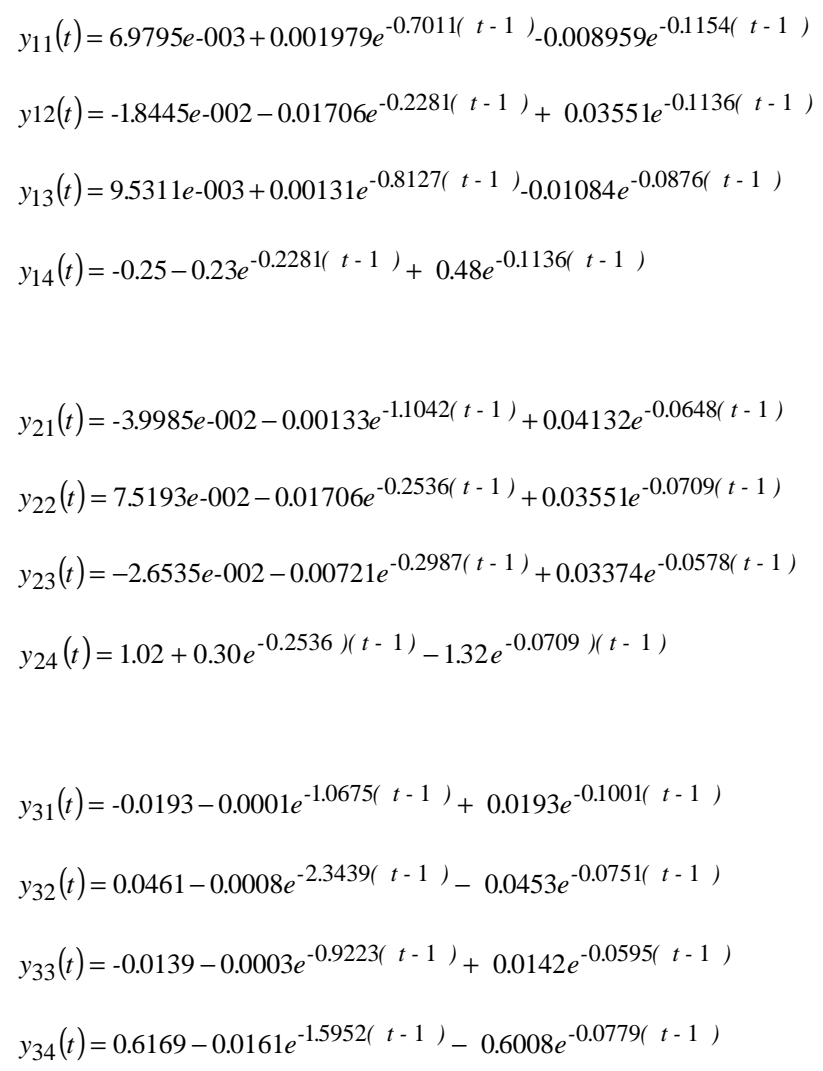

\section{Para o modelo da planta industrial:}

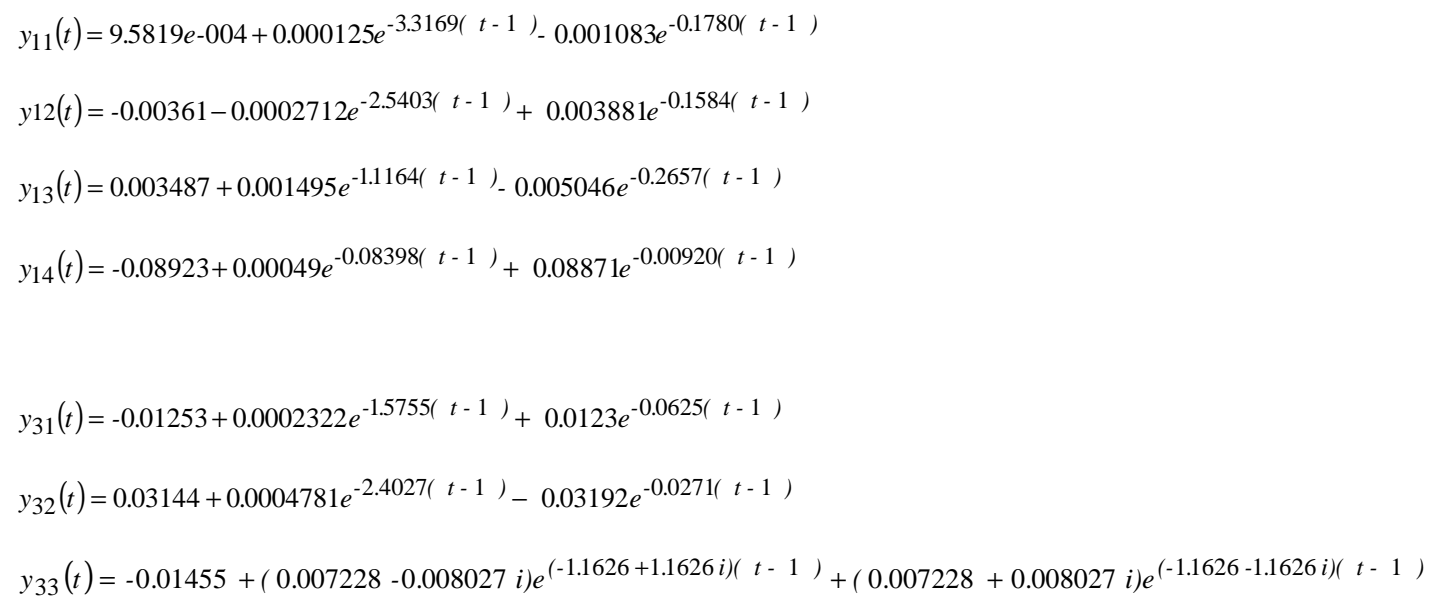


$y_{34}(t)=0.6675+(-0.3337+0.5133 i) e^{(-0.3007-0.1887 i)(t-1)}+(-0.3337-0.5133 i) e^{(-0.3007+0.1887 i)(t-1)}$

Nas equações acima $y_{\mathrm{ij}}(t)$ representa uma resposta da saída $i$ no instante $t$ para um degrau unitário aplicado na entrada $j$ no instante 0 , onde:

$\mathrm{i}=1$ - representa a concentração de $\mathrm{C}_{3}^{-}$na corrente de fundo da coluna.

$\mathrm{i}=2$ - representa a concentração de $\mathrm{C}_{4}{ }^{+}$na corrente de topo da coluna.

$\mathrm{i}=3$ - representa a temperatura de topo da coluna.

$\mathrm{j}=1$ - representa a vazão de refluxo de topo da coluna.

$\mathrm{j}=2$ - representa a vazão de fluido de aquecimento para o refervedor da coluna.

j = 3 - representa a vazão de carga para a coluna.

$\mathrm{j}=4$ - representa a temperatura do fluido de aquecimento para refervedor. 


\section{8}

\section{ALGORITMOS DE CONTROLE}

As principais características dos controladores preditivos inicialmente desenvolvidos por Cutler e Ramaker (1979), Prett e Gillette (1980) e Richalet et al. (1978) são as seguintes:

- Embora na engenharia de controle clássico, o modelo do processo seja definido por um modelo de estado ou função de transferência, nos controladores preditivos, as saídas futuras são previstas usando modelos de resposta ao degrau ou impulso.

- Diante das incertezas no modelo identificado, variações da planta e perturbações não medidas, introduziu-se o termo de correção da predição como a diferença do valor estimado e o valor medido.

- As ações de controle minimizam um índice de performance definido pelo usuário. Normalmente é minimizada a diferença quadrática entre a trajetória desejada e a trajetória prevista.

- As ações de controle são normalmente calculadas através de um algoritmo de otimização, onde incluímos pesos e restrições ao problema de controle.

- O algoritmo de controle calcula a trajetória futura das entradas, porém somente a primeira ação de controle será implementada na planta.

Nos controladores preditivos industriais as funções são executadas em um ciclo de amostragem pré-definido onde temos as seguintes etapas:

- Leitura das entradas e saídas da planta;

- Cálculo das predições das saídas;

- Cálculo do erro em relação à trajetória desejada;

- Cálculo das ações de controle;

- Implementação das ações de controle na planta.

A seguir apresentaremos a forma de evolução do MPC onde serão abordados os seguintes pontos: 
- $\quad$ MPC com modelo não-paramétrico;

- MPC com modelo de ordem reduzida;

- $\quad$ MPC com multi-modelos;

- Solução do algoritmo MPC com SQP.

\section{1 - MPC com modelo não-paramétrico}

Neste tipo de algoritmo é utilizado um modelo FIR (finite impulse response) ou um modelo de resposta ao degrau para representar o comportamento dinâmico do processo. $\mathrm{O}$ modelo é obtido como uma seqüência de coeficientes numéricos que relaciona as entradas com as saídas. Por exemplo, no caso do modelo FIR temos a saída $i$ relacionada com a entrada $j$ da seguinte forma:

$$
\Delta y_{i}(k)=\sum_{n=1}^{n b_{i, j}} b_{i, j, n} \Delta u_{j}(n)
$$

Sendo:

$n b_{i, j}$ - número de coeficientes do modelo

$b_{i, j, n}$ - corresponde ao n-ésimo coeficiente da resposta ao pulso unitário

\subsection{1 - Cálculo das predições das variáveis controladas}

As predições das variáveis controladas são baseadas no modelo do sistema e nas ações de controle já aplicadas ao processo.

O cálculo das predições tem as seguintes etapas:

- Cálculo do efeito da última ação de controle;

- Correção da predição através das leituras da planta real;

- Translação da predição corrigida.

\subsubsection{1 - Efeito da última ação de controle}


No caso de um processo estável em malha aberta, podemos representar a predição da variável controlada como a composição de duas parcelas, uma parte corresponde aos efeitos das entradas passadas e a outra parte referente à contribuição da última ação de controle, esta predição pode ser representada da seguinte forma:

$$
\left[y_{i, k+n}\right]_{k / k-1}=\left[y_{i, k+n}\right]_{k-1 / k-1}+\sum_{j=1}^{n u} s_{i, j, n} \Delta u_{j, k-1}
$$

sendo:

$$
\begin{aligned}
& n \quad \text { - instante futuro } n=1,2, \ldots \\
& {\left[y_{i, k+n}\right]_{k-1 / k-1} \text { - predição da variável controlada } \boldsymbol{i} \text { no instante futuro } \boldsymbol{k + n}} \\
& \text { calculada no instante } \boldsymbol{k}-\mathbf{1} \text { com informações do processo até } \boldsymbol{k} \text {-1. } \\
& {\left[y_{i, k+n}\right]_{k / k-1} \quad \text { - predição da variável controlada } \boldsymbol{i} \text { no instante futuro } \boldsymbol{k}+\boldsymbol{n}} \\
& \text { calculada no instante } \boldsymbol{k} \text { com informações do processo até } \boldsymbol{k}-\mathbf{1} \text {. } \\
& s_{i, j, n} \quad \text { - coeficiente correspondente ao instante } \boldsymbol{n} \text { da resposta ao degrau } \\
& \text { da variável controlada } \boldsymbol{i} \text { com a variável manipulada } \boldsymbol{j} \\
& \Delta u_{j, k-1}=u_{j, k-1}-u_{j, k-2} \\
& u_{j, k-1} \quad-\text { valor da variável manipulada } \mathbf{j} \text { aplicada no instante } \boldsymbol{k}-\mathbf{1} \text {. } \\
& u_{j, k-2} \quad-\text { valor da variável manipulada } \mathbf{j} \text { aplicada no instante } \boldsymbol{k}-\mathbf{2} \text {. }
\end{aligned}
$$

Usando (8.1) para calcular as predições de todas as variáveis controladas, temos:

$$
\left[y_{k+n}\right]_{k / k-1}=\left[y_{k+n}\right]_{k-1 / k-1}+S_{n} \Delta u_{k-1}
$$

Sendo:

$$
\begin{array}{ll}
{\left[y_{k+n}\right]_{k-1 / k-1}=\left[\left[y_{1, k+n}\right]_{k-1 / k-1} \cdots\left[y_{n y, k+n}\right]_{k-1 / k-1}\right]^{T}} & {\left[y_{k+n}\right]_{k-1 / k-1} \in \Re^{n y \times 1}} \\
{\left[y_{k+n}\right]_{k / k-1}=\left[\left[y_{1, k+n}\right]_{k / k-1} \cdots\left[y_{n y, k+n}\right]_{k / k-1}\right]^{T}} & {\left[y_{k+n}\right]_{k / k-1} \in \Re^{n y \times 1}} \\
\Delta u_{k-1}=\left[\Delta u_{1, k-1} \cdots \Delta u_{n u, k-1}\right]^{T} & \Delta u_{k-1} \in \mathfrak{R}^{n u \times 1}
\end{array}
$$




$$
S_{n}=\left[\begin{array}{ccc}
S_{1,1, n} & \cdots & s_{1, n u, n} \\
\vdots & & \vdots \\
s_{n y, 1, n} & \cdots & s_{n y, n u, n}
\end{array}\right] \quad S_{n} \in \Re^{n y \times n u}
$$

sendo, $\boldsymbol{n} \boldsymbol{u}$ o número de variáveis de entrada (manipuladas e perturbações) e $\boldsymbol{n y}$ o número de variáveis de saída (controladas)

No enfoque convencional as predições das variáveis controladas são feitas em instantes sequiênciais defasados de um período de amostragem até o maior horizonte de estabilização dos modelos, ou seja:

$$
\mathrm{np}=\max _{i, j}\left(n h_{i, j}\right)
$$

Assim, as predições das variáveis controladas em todos os instantes desejados, podem ser apresentadas da seguinte forma:

$$
[y]_{k / k-1}=[y]_{k-1 / k-1}+S \Delta u_{k-1}
$$

sendo:

$$
\begin{aligned}
& \left.[y]_{k-1 / k-1}=\left[\begin{array}{llll}
{\left[y_{k+1}\right]_{k-1 / k-1}^{T}} & {\left[y_{k+2}\right]_{k-1 / k-1}^{T}} & \cdots & {\left[y_{k+n p}\right.}
\end{array}\right]_{k-1 / k-1}^{T}\right]^{T} \\
& {[y]_{k / k-1}=\left[\begin{array}{llll}
{\left[y_{k+1}\right]_{k / k-1}^{T}} & {\left[y_{k+2}\right]_{k / k-1}^{T}} & \cdots & {\left[y_{k+n p}\right]_{k / k-1}^{T}}
\end{array}\right]^{T}} \\
& \Delta u_{k-1}=\left[\Delta u_{1, k-1} \cdots \Delta u_{n u, k-1}\right]^{T} \\
& S=\left[\begin{array}{llll}
S_{1}^{T} & S_{2}^{T} & \cdots & S_{n p}^{T}
\end{array}\right]^{T}
\end{aligned}
$$

\subsubsection{2 - Correção das predições com as leituras da planta}

Diante das incertezas em relação aos modelos da planta e perturbações não medidas, é necessário acrescentar um termo de correção das predições das variáveis controladas.

Com os efeitos das ações de controle calculados, devemos corrigir as predições através da leitura das variáveis controladas. As predições dessas variáveis, no primeiro instante deveriam corresponder aos valores lidos das variáveis controladas (se os 
modelos fossem perfeitos). Para o cálculo dessa correção, utilizamos apenas os termos correspondentes ao instante $\boldsymbol{k}+\boldsymbol{1}$ do vetor de predições, ou seja:

$$
\left[y_{k+1}\right]_{k / k-1}=N[y]_{k / k-1}
$$

sendo:

$$
\begin{aligned}
& {\left[y_{k+1}\right]_{k / k-1}=\left[\left[y_{1, k+1}\right]_{k / k-1} \quad\left[y_{2, k+1}\right]_{k / k-1} \quad \cdots \quad\left[y_{n y, k+1}\right]_{k / k-1}\right]^{T}} \\
& N=\left[\begin{array}{ccccccc}
1 & 0 & \cdots & 0 & 0 & \cdots & 0 \\
0 & 1 & \cdots & 0 & 0 & \cdots & 0 \\
\vdots & \vdots & \ddots & \vdots & \vdots & & \vdots \\
0 & 0 & \cdots & 1 & 0 & \cdots & 0
\end{array}\right] ; \quad N \in \mathfrak{R}^{n y \times n y . n p}
\end{aligned}
$$

Considerando a leitura da variável controlada $\boldsymbol{i}$ representada por $\hat{y}_{i, k}$, podemos calcular o desvio da predição em relação à leitura da planta como

$$
w_{i, k}=\hat{y}_{i, k}-\left[y_{i, k+1}\right]_{k / k-1}
$$

Para todas as variáveis controladas, obtemos:

$$
W_{k}=\hat{y}_{k}-\left[y_{k+1}\right]_{k / k-1}
$$

sendo:

$$
\begin{array}{rlrl}
W_{k} & =\left[\begin{array}{lll}
w_{1, k} & \cdots & w_{n y, k}
\end{array}\right]^{T} & & W_{k} \in \Re^{n y \times 1} \\
\hat{y}_{k} & =\left[\begin{array}{lll}
\hat{y}_{1, k} & \cdots & \hat{y}_{n y, k}
\end{array}\right]^{T} & \hat{y}_{k} \in \mathfrak{R}^{n y \times 1}
\end{array}
$$

Substituindo (8.4) em (8.5), temos:

$$
W_{k}=\hat{y}_{k}-N[y]_{k / k-1}
$$

A correção deste desvio é normalmente propagada para todas as predições futuras. A lei de propagação da correção adotada neste trabalho é apresentada como: 


$$
\left\lfloor y_{i, k+n}^{c}\right\rfloor_{k / k-1}=\left[y_{i, k+n}\right]_{k / k-1}+w_{i, k}
$$

sendo:

$$
\begin{array}{r}
{\left[y_{i, k+n}^{c}\right]_{k / k-1} \quad \text { - predição corrigida da variável controlada } \boldsymbol{i} \text { no instante futuro }} \\
\boldsymbol{k}+\boldsymbol{n} \text { calculado no instante } \boldsymbol{k} \text { com informações do processo em }
\end{array}
$$

k-1.

Podemos escrever as predições corrigidas para todas as variáveis controladas e todos os instantes futuros escolhidos, assim temos:

$$
\left[y^{c}\right]_{k / k-1}=[y]_{k / k-1}+K W_{k}
$$

sendo:

$$
K=\left[\begin{array}{cccc}
1 & 0 & \cdots & 0 \\
0 & 1 & \cdots & 0 \\
\vdots & \vdots & \ddots & \vdots \\
0 & 0 & \cdots & 1 \\
1 & 0 & \cdots & 0 \\
\vdots & \vdots & & \vdots \\
0 & 0 & \cdots & 1 \\
\vdots & \vdots & & \vdots \\
1 & 0 & \cdots & 0 \\
\vdots & \vdots & & \vdots \\
0 & 0 & \cdots & 1
\end{array}\right]
$$

Substituindo as equações (8.3) e (8.6) em (8.7), temos:

$$
\left[y^{c}\right]_{k / k-1}=[y]_{k-1 / k-1}+S \Delta u_{k-1}+K\left\{\hat{y}_{k}-N\left([y]_{k-1 / k-1}+S \Delta u_{k-1}\right)\right\}
$$

Agrupando os termos, temos:

$$
\left[y^{c}\right]_{k / k-1}=(I-K N)[y]_{k-1 / k-1}+K \hat{y}_{k}+(I-K N) S \Delta u_{k-1}
$$


sendo:

$$
\begin{aligned}
& I=\left[\begin{array}{cccc}
I_{n y} & 0 & \cdots & 0 \\
0 & I_{n y} & \cdots & 0 \\
\vdots & \vdots & \ddots & \vdots \\
0 & 0 & \cdots & I_{n y}
\end{array}\right] \\
& I_{n y}=\left[\begin{array}{cccc}
1 & 0 & \cdots & 0 \\
0 & 1 & \cdots & 0 \\
\vdots & \vdots & \ddots & \vdots \\
0 & 0 & \cdots & 1
\end{array}\right]
\end{aligned}
$$

\subsubsection{3 - Translação da predição corrigida}

As predições das variáveis controladas estão atualizadas e corrigidas para o instante $\boldsymbol{k}$. Isto significa que:

$$
\begin{gathered}
{\left[y_{i, k+2}^{c}\right]_{k / k-1} \text { - corresponde ao instante atual } \boldsymbol{k + 1}} \\
{\left[y_{i, k+3}^{c}\right]_{k / k-1}-\text { corresponde ao instante futuro } \boldsymbol{k}+\boldsymbol{2}} \\
\vdots \\
{\left[y_{i, k+n p+1}^{c}\right]_{k / k-1}-\text { corresponde ao instante futuro } \boldsymbol{k}+\boldsymbol{n p}}
\end{gathered}
$$

Portanto, dentro da filosofia dos controladores preditivos, que apresentam um horizonte móvel, é necessário fazer a translação de um período de amostragem em todo vetor de predições e para todas as variáveis controladas. 


$$
\begin{gathered}
{\left[y_{1, k+1}\right]_{k / k}=\left[y_{1, k+2}^{c}\right]_{k / k-1}} \\
\vdots \\
{\left[y_{n y, k+1}\right]_{k}=\left[y_{n y, k+2}^{c}\right]_{k / k-1}} \\
{\left[y_{1, k+2}\right]_{k / k}=\left[y_{1, k+3}^{c}\right]_{k / k-1}} \\
\vdots \\
{\left[y_{n y, k+2}\right]_{k / k}=\left[y_{n y, k+3}^{c}\right]_{k / k-1}} \\
\vdots \\
{\left[y_{1, k+n p-1}\right]_{k / k}=\left[y_{1, k+n p}^{c}\right]_{k / k-1}} \\
\vdots \\
{\left[y_{n y, k+n p-1}\right]_{k / k}=\left[y_{n y, k+n p}^{c}\right]_{k / k-1}}
\end{gathered}
$$

Para sistemas estáveis, assume-se que $\boldsymbol{n p}$ é suficientemente grande tal que:

$$
\left[y_{i, n p}\right]_{k / k}=\left[y_{i, n p}^{c}\right]_{k / k-1}
$$

Vetorialmente, podemos representar a translação da seguinte forma:

$$
[y]_{k / k}=M\left[y^{c}\right]_{k / k-1}
$$

sendo:

$$
M=\left[\begin{array}{ccccc}
0 & I_{n y} & 0 & \cdots & 0 \\
0 & 0 & I_{n y} & \cdots & 0 \\
\vdots & \vdots & \vdots & \ddots & \vdots \\
0 & 0 & 0 & \cdots & I_{n y} \\
0 & 0 & 0 & \cdots & I_{n y}
\end{array}\right] ; \quad M \in \Re^{n y . n p \times n y . n p}
$$

Substituindo a eq. (8.8) na eq. (8.9), obtemos a seguinte expressão para as predições das variáveis controladas.

$$
[y]_{k / k}=M(I-K N)[y]_{k-1 / k-1}+M K \hat{y}_{k}+M(I-K N) S \Delta u_{k-1}
$$

\subsection{2 - Cálculo das ações de controle}


O algoritmo de controle preditivo calcula as ações de controle segundo as seguintes etapas:

- Recebe a trajetória referência para as variáveis controladas em determinados instantes futuros. O número de instantes é denominado horizonte de predição (np).

- Calcula os erros das variáveis controladas em relação ao setpoint ou a uma faixa de operação, onde o erro é nulo quando a predição está dentro dos limites estabelecidos para as variáveis.

- Calcula os valores das ações de controle nos instantes futuros de modo a minimizar os erros das variáveis controladas em certos instantes futuros.

A seguir serão descritas estas etapas.

\subsubsection{1 - Trajetória de referência das variáveis controladas}

Calculadas as predições das variáveis controladas, utilizam-se estas mesmas no cálculo das ações de controle. Na forma tradicional de cálculo do controlador preditivo o horizonte de predição (np) corresponde aos instantes futuros até o maior tempo de estabilização dos modelos do processo. O horizonte de otimização (nr) é relativo aos instantes futuros em que o controlador irá minimizar os erros.

Para o cálculo das ações de controle, utilizamos apenas os termos correspondentes até o instante $\boldsymbol{k}+\boldsymbol{n} \boldsymbol{r}$ do vetor de predições, pois comumente não é interessante calcularmos as ações de controle considerando todos os instantes até o estado estacionário. Desta forma, podemos escrever:

$$
[\tilde{y}]_{k / k}=N_{r}[y]_{k / k}
$$

sendo:

$$
[\tilde{y}]_{k / k}=\left[\begin{array}{llll}
{\left[y_{k+1}\right]_{k / k}^{T}} & {\left[y_{k+2}\right]_{k / k}^{T}} & \cdots & {\left[y_{k+n r}\right]_{k / k}^{T}}
\end{array}\right]^{T}
$$




$$
N_{r}=\left[\begin{array}{ccccccc}
1 & 0 & \cdots & 0 & 0 & \cdots & 0 \\
0 & 1 & \cdots & 0 & 0 & \cdots & 0 \\
\vdots & \vdots & \ddots & \vdots & \vdots & & \vdots \\
0 & 0 & \cdots & 1 & 0 & \cdots & 0
\end{array}\right] \quad N_{r} \in \Re^{n r . n y \times n p . n y}
$$

As predições das variáveis controladas são alteradas ao aplicarmos as ações de controle em cada instante. Os controladores preditivos têm a característica de poder calcular mais de uma ação de controle, que fornece mais graus de liberdade para o controlador preditivo. Quando aumentamos o número de ações calculadas, permitimos ao controlador distribuir de forma mais conveniente as suas ações. Desta forma, quando aplicamos “ $\boldsymbol{m}$ ” ações de controle, temos:

$$
\begin{aligned}
& {\left[\bar{y}_{k+1}\right]_{k / k}=\left[y_{k+1}\right]_{k / k}+S_{1} \Delta u_{k}} \\
& {\left[\bar{y}_{k+2}\right]_{k / k}=\left[y_{k+2}\right]_{k / k}+S_{2} \Delta u_{k}+S_{1} \Delta u_{k+1}} \\
& \vdots \\
& {\left[\bar{y}_{k+m}\right]_{k / k}=\left[y_{k+m}\right]_{k / k}+S_{m} \Delta u_{k}+S_{m-1} \Delta u_{k+1}+\cdots+S_{1} \Delta u_{k+m-1}} \\
& \vdots \\
& {\left[\bar{y}_{k+n r}\right]_{k / k}=\left[y_{k+n r}\right]_{k / k}+S_{n r} \Delta u_{k}+S_{n r-1} \Delta u_{k+1}+\cdots+S_{n r-m+1} \Delta u_{k+m-1}}
\end{aligned}
$$

$\mathrm{Na}$ forma vetorial, temos:

$$
[\bar{y}]_{k / k}=[\tilde{y}]_{k / k}+S_{m} \Delta u
$$

sendo:

$$
\begin{aligned}
& {[\bar{y}]_{k / k}=\left[\begin{array}{llllll}
\bar{y}_{k+1} & \bar{y}_{k+2} & \cdots & \bar{y}_{k+m} & \cdots & \bar{y}_{k+n r}
\end{array}\right]_{k / k}^{T}} \\
& \Delta u=\left[\begin{array}{lll}
\Delta u_{k} & \cdots & \Delta u_{k+m-1}
\end{array}\right]^{T} \\
& S_{m}=\left[\begin{array}{cccc}
S_{1} & 0 & \cdots & 0 \\
S_{2} & S_{1} & \cdots & 0 \\
\vdots & \vdots & & \vdots \\
S_{m} & S_{m-1} & \cdots & S_{1} \\
\vdots & \vdots & & \vdots \\
S_{n r} & S_{n r-1} & \cdots & S_{n r-m+1}
\end{array}\right]
\end{aligned}
$$




\subsubsection{2 - Cálculo dos erros das variáveis controladas}

Tendo a trajetória prevista das variáveis controladas, podemos calcular os erros em relação aos respectivos setpoints. Assim, para a variável controlada $i$ no instante futuro $\boldsymbol{j}$, o erro é definido como:

$$
\left[e_{i, k+j}\right]_{k / k}=y_{s p_{i}}-\left[y_{i, k+j}\right]_{k / k}
$$

sendo:

$y_{s p_{i}}$ - é o setpoint correspondente à variável controlada $\boldsymbol{i}$

Podemos então definir um vetor de setpoints dado por:

$$
\left[e_{k+j}\right]_{k / k}=I_{n y} y_{s p}-\left[y_{k+j}\right]_{k / k}
$$

sendo:

$$
\begin{aligned}
& {\left[e_{k+j}\right]_{k / k}=\left[\begin{array}{lll}
e_{1, k+j} & \cdots & e_{n y, k+j}
\end{array}\right]_{k / k}^{T} \quad ; \quad\left[e_{k+j}\right]_{k / k} \in \mathfrak{R}^{n y \times 1}} \\
& {\left[y_{k+j}\right]_{k / k}=\left[\begin{array}{lll}
y_{1, k+j} & \cdots & y_{n y, k+j}
\end{array}\right]_{k / k}^{T} \quad ; \quad\left[y_{k+j}\right]_{k / k} \in \mathfrak{R}^{n y \times 1}} \\
& y_{s p}=\left[\begin{array}{lll}
y_{s p_{1}} & \cdots & y_{s p_{n y}}
\end{array}\right]^{T} \quad ; \quad y_{s p} \in \mathfrak{R}^{n y \times 1}
\end{aligned}
$$

Para todos os instantes de predição futuros, temos:

$$
[e]_{k / k}=\tilde{N} y_{s p}-[\tilde{y}]_{k / k}
$$

sendo:

$$
\begin{aligned}
& {[e]_{k / k}=\left[\begin{array}{llll}
e_{k+1} & e_{k+2} & \cdots & e_{k+n r}
\end{array}\right]^{T}} \\
& y_{s p}=\left[\begin{array}{lll}
y_{s p_{1}} & \cdots & y_{s p_{n y}}
\end{array}\right]^{T}
\end{aligned}
$$




$$
\tilde{N}=\left[\begin{array}{ccc}
1 & \cdots & 0 \\
\vdots & \ddots & \vdots \\
0 & \cdots & 1 \\
1 & \cdots & 0 \\
\vdots & \ddots & \vdots \\
0 & \cdots & 1 \\
\vdots & & \vdots \\
1 & \cdots & 0 \\
\vdots & \ddots & \vdots \\
0 & \cdots & 1
\end{array}\right] \quad ; \quad \tilde{N} \in \Re^{\text {ny.nrxny }}
$$

\subsubsection{3 - Algoritmo de controle preditivo}

Os controladores de forma geral têm como principal objetivo minimizar os erros das variáveis controladas com os menores movimentos das variáveis manipuladas.

No caso do controlador preditivo o objetivo é minimizar a seguinte função:

$$
J_{k}=[\bar{e}]_{k / k}^{T} \Gamma[\bar{e}]_{k / k}+\Delta u^{T} \Lambda \Delta u
$$

sendo:

$$
\begin{aligned}
& {[\bar{e}]_{k / k}=[e]_{k / k}-S_{m} \Delta u} \\
& {[\bar{e}]_{k / k}=\left[\begin{array}{llll}
\bar{e}_{k+1} & \bar{e}_{k+2} & \cdots & \bar{e}_{k+n r}
\end{array}\right]^{T}}
\end{aligned}
$$

$\Gamma$ é uma matriz de pesos para os erros nas variáveis controladas

$\Lambda$ é uma matriz de pesos para os movimentos das variáveis manipuladas

$$
\begin{aligned}
\Gamma & =\left[\begin{array}{cccc}
\underline{\Gamma} & 0 & \cdots & 0 \\
0 & \underline{\Gamma} & \cdots & 0 \\
\vdots & \vdots & \ddots & \vdots \\
0 & 0 & \cdots & \underline{\Gamma}
\end{array}\right] \quad ; \quad \Gamma \in \Re^{n y . n r \times n y . n r} \\
\underline{\Gamma} & =\left[\begin{array}{ccc}
\gamma_{1} & \cdots & 0 \\
\vdots & \ddots & \vdots \\
0 & \cdots & \gamma_{n y}
\end{array}\right] \quad ; \quad \Gamma \in \Re^{n y \times n y}
\end{aligned}
$$




$$
\begin{array}{rlrl}
\Lambda & =\left[\begin{array}{ccc}
\underline{\Lambda} & \cdots & 0 \\
\vdots & \ddots & \vdots \\
0 & \cdots & \Lambda
\end{array}\right] \quad ; \quad & \Lambda \in \mathfrak{R}^{m \cdot n u \times m . n u} \\
\underline{\Lambda} & =\left[\begin{array}{ccc}
\lambda_{1} & \cdots & 0 \\
\vdots & \ddots & \vdots \\
0 & \cdots & \lambda_{n u}
\end{array}\right] & &
\end{array}
$$

Para solucionar o problema, devemos desenvolver o equacionamento da função objetivo do controlador. Desta forma, podemos substituir a equação (8.14) em (8.13), resultando em:

$$
\begin{aligned}
& J_{k}=\left([e]_{k / k}-S_{m} \Delta u\right)^{T} \Gamma\left([e]_{k / k}-S_{m} \Delta u\right)+\Delta u^{T} \Lambda \Delta u \\
& J_{k}=[e]_{k / k}^{T} \Gamma[e]_{k / k}-\Delta u^{T} S_{m}^{T} \Gamma[e]_{k / k}-[e]_{k / k}^{T} \Gamma S_{m} \Delta u+\Delta u^{T}\left(S_{m}^{T} \Gamma S_{m}+\Lambda\right) \Delta u
\end{aligned}
$$

Os algoritmos de controle preditivo convencionais se diferenciam na forma de minimizar a função objetivo.

Como exemplo descreveremos os algoritmos: DMC, QDMC e MMPC

\subsubsection{1 - Algoritmo DMC}

O algoritmo DMC é o de abordagem mais simples, e se baseia no método dos mínimos quadrados.

Sua função objetivo é dada pela eq. (8.15):

$$
J_{k}=[e]_{k / k}^{T} \Gamma[e]_{k / k}-\Delta u^{T} S_{m}^{T} \Gamma[e]_{k / k}-[e]_{k / k}^{T} \Gamma S_{m} \Delta u+\Delta u^{T}\left(S_{m}^{T} \Gamma S_{m}+\Lambda\right) \Delta u
$$

Como os termos são todos escalares, podemos dizer que

$$
\Delta u^{T} S_{m}^{T} \Gamma[e]_{k / k}=[e]_{k / k}^{T} \Gamma S_{m} \Delta u
$$


portanto:

$$
J_{k}=[e]_{k / k}^{T} \Gamma[e]_{k / k}-2 \Delta u^{T} S_{m}^{T} \Gamma[e]_{k / k}+\Delta u^{T}\left(S_{m}^{T} \Gamma S_{m}+\Lambda\right) \Delta u
$$

O valor mínimo da função objetivo corresponde ao ponto onde a derivada da função em relação ao $\Delta \boldsymbol{u}$ é zero, isto é:

$$
\frac{\partial J_{k}}{\partial \Delta u}=0
$$

Portanto:

$$
\frac{\partial J_{k}}{\partial \Delta u}=-2 S_{m}^{T} \Gamma[e]_{k / k}+2\left(S_{m}^{T} \Gamma S_{m}+\Lambda\right) \Delta u
$$

como $\frac{\partial J_{k}}{\partial \Delta u}=0$, portanto:

$$
0=-2 S_{m}^{T} \Gamma[e]_{k / k}+2\left(S_{m}^{T} \Gamma S_{m}+\Lambda\right) \Delta u
$$

Isolando o termo em $\Delta \boldsymbol{u}$, temos:

$$
\Delta u=\left(S_{m}^{T} \Gamma S_{m}+\Lambda\right)^{-1} S_{m}^{T} \Gamma[e]_{k / k}
$$

Podemos escrever a equação do controlador na forma

$$
\Delta u=K_{M P C}[e]_{k / k}
$$

sendo:

$$
K_{M P C}=\left(S_{m}^{T} \Gamma S_{m}+\Lambda\right)^{-1} S_{m}^{T} \Gamma
$$

\subsubsection{2 - Algoritmo QDMC}

O algoritmo QDMC utiliza o método de otimização de programação quadrática (QP) para encontrar a melhor solução para o problema de controle.

De forma compacta podemos representar o problema da programação quadrática da seguinte maneira: 


$$
\begin{array}{cc}
\underset{\Delta u}{\text { Minimizar }} & \Phi=\frac{1}{2} x^{T} H x+C^{T} x \\
\text { sujeito } a & A x \geq b \\
& x \geq 0
\end{array}
$$

Para resolvermos o controlador por QP, partimos da função objetivo básica, resultando numa forma equivalente à eq.(8.15):

$$
J_{k}=[e]_{k / k}^{T} \Gamma[e]_{k / k}-2[e]_{k / k}^{T} \Gamma S_{m} \Delta u+\Delta u^{T}\left(S_{m}^{T} \Gamma S_{m}+\Lambda\right) \Delta u
$$

O termo $[e]_{k / k}^{T} \Gamma[e]_{k / k}$ na função objetivo $\boldsymbol{J}_{\boldsymbol{k}}$ é uma constante escalar. Neste caso, podemos eliminar este termo da função sem alterar a sua solução final. Também podemos dividir a função objetivo por 2 sem alterar a sua solução.

Assim, a função objetivo do QDMC pode ser apresentada da seguinte forma:

$$
\Phi=\frac{1}{2} \Delta u^{T}\left(S_{m}^{T} \Gamma S_{m}+\Lambda\right) \Delta u-[e]_{k / k}^{T} \Gamma S_{m} \Delta u
$$

De forma simplificada podemos representar $\Phi$ como:

$$
\Phi=\frac{1}{2} \Delta u^{T} H \Delta u+C^{T} \Delta u
$$

sendo:

$$
\begin{aligned}
& H=S_{m}^{T} \Gamma S_{m}+\Lambda \\
& C^{T}=-[e]_{k}^{T} \Gamma S_{m}
\end{aligned}
$$

Na programação quadrática, podemos incluir restrições do tipo:

$$
A \Delta u \geq b
$$

Neste caso, pode-se incluir as seguintes restrições: 
1. Restrições de movimento das variáveis manipuladas

2. Restrições de limites das variáveis manipuladas

\subsection{1 - Restrições de movimentos}

Estas restrições podem ser apresentadas da seguinte forma:

$$
-\Delta u_{\max } \leq \Delta u_{k+j} \leq \Delta u_{\max }
$$

Para o caso $\Delta u_{k+j} \leq \Delta u_{\max } \operatorname{com} j=1,2, \ldots, m$, temos

$$
I_{m . n u} \Delta u \leq N_{u} \Delta u_{\max }
$$

sendo:

$$
\begin{gathered}
N_{u}=\left[\begin{array}{c}
I_{n u} \\
\vdots \\
I_{n u}
\end{array}\right] ; \quad N_{u} \in \Re^{m . n u \times n u} \\
\text { Para o caso }-\Delta u_{\max } \leq \Delta u_{k+j} \text { temos } \\
-\Delta u_{k+j} \leq \Delta u_{\max } \\
-I_{m . n u} \Delta u \leq N_{u} \Delta u_{\max }
\end{gathered}
$$

compondo as duas condições, temos:

$$
\left[\begin{array}{c}
I_{m . n u} \\
-I_{m . n u}
\end{array}\right] \Delta u \leq\left[\begin{array}{c}
N_{u} \Delta u_{\max } \\
N_{u} \Delta u_{\max }
\end{array}\right]
$$

\subsection{2 - Restrições de limites}

As restrições de limites podem ser apresentadas da seguinte forma: 


$$
u_{\min } \leq u \leq u_{\max }
$$

como:

$$
\left[\begin{array}{c}
u_{k} \\
u_{k+1} \\
\vdots \\
u_{k+m-1}
\end{array}\right]=\left[\begin{array}{cccc}
I_{n u} & 0 & \cdots & 0 \\
I_{n u} & I_{n u} & \cdots & 0 \\
\vdots & \vdots & \ddots & \vdots \\
I_{n u} & I_{n u} & \cdots & I_{n u}
\end{array}\right]\left[\begin{array}{c}
\Delta u_{k} \\
\Delta u_{k+1} \\
\vdots \\
\Delta u_{k+m-1}
\end{array}\right]+\left[\begin{array}{c}
u_{k-1} \\
u_{k-1} \\
\vdots \\
u_{k-1}
\end{array}\right]
$$

ou

$$
u=\bar{N}_{u} \Delta u+u_{k-1}
$$

Para o caso $u \leq u_{\max }$

$$
\begin{aligned}
& \bar{N}_{u} \Delta u+u_{k-1} \leq u_{\max } \\
& \bar{N}_{u} \Delta u \leq u_{\max }-u_{k-1}
\end{aligned}
$$

Para o caso $u_{\min } \leq u$

$$
\begin{aligned}
& u_{\min } \leq \bar{N}_{u} \Delta u+u_{k-1} \\
& -\bar{N}_{u} \Delta u \leq u_{k-1}-u_{\min }
\end{aligned}
$$

compondo as duas condições, temos:

$$
\left[\begin{array}{c}
\bar{N}_{u} \\
-\bar{N}_{u}
\end{array}\right] \Delta u \leq\left[\begin{array}{l}
u_{\max }-u_{k-1} \\
u_{k-1}-u_{\min }
\end{array}\right]
$$

compondo todas as restrições, temos:

$$
\left[\begin{array}{l}
I_{m \cdot n u} \\
-I_{m \cdot n u} \\
\bar{N}_{u} \\
-\bar{N}_{u}
\end{array}\right] \Delta u \leq\left[\begin{array}{l}
N_{u} \Delta u_{\max } \\
N_{u} \Delta u_{\max } \\
u_{\max }-u_{k-1} \\
u_{k-1}-u_{\min }
\end{array}\right]
$$

Portanto:

$$
A \Delta u \leq b
$$

sendo: 


$$
A=\left[\begin{array}{l}
I_{m . n u} \\
-I_{m . n u} \\
\bar{N}_{u} \\
-\bar{N}_{u}
\end{array}\right] ; \quad b=\left[\begin{array}{l}
N u \Delta u_{\max } \\
N u \Delta u_{\max } \\
u_{\max }-u_{k-1} \\
u_{k-1}-u_{\min }
\end{array}\right]
$$

\section{2 - MPC com modelo de ordem reduzida}

Os MPC's convencionais necessitam de todos os estados do processo até o estado estacionário, para poder representar corretamente as previsões das variáveis controladas. Algumas tentativas de reduzir a quantidade de estados foram apresentadas por Hovd et al. (1993), que propõem truncar o modelo de convolução antes de atingir o estado estacionário e Li et al. (1989) que transformam um modelo não paramétrico em um modelo em espaço de estado.

Entretanto, uma solução inovadora de redução de ordem do modelo foi proposta por Odloak (1996) e Gouvêa e Odloak (1997), na qual o modelo de estado é obtido a partir de um modelo de função de transferência.

\subsection{1 - Modelo de função de transferência}

Os modelos do processo, originalmente eram representados através das respostas das variáveis controladas a degraus nas variáveis de entrada (manipuladas e perturbações medidas). Obtinham-se estes modelos através do ajuste dos dados de um teste na planta a um modelo do tipo FIR (Finite Impulse Response).

Os processos químicos contínuos quando próximos de suas condições normais de projeto são normalmente bem representados por modelos lineares do tipo função de transferência. Conforme Ljung (1987) seria interessante fazer uma representação do processo através de modelos paramétricos de ordem reduzida. Portanto, para um sistema multivariável, a relação dinâmica entre a i-ésima variável controlada (ou saída) e a jésima variável manipulada (ou entrada), pode ser colocada na forma:

$$
G_{i, j}(s)=\frac{b_{i, j}(s)}{1+a_{i, j}(s)} e^{-s \theta_{i, j}}
$$


sendo:

$$
\begin{aligned}
& b_{i, j}(s)=b_{i, j, 0}+b_{i, j, 1} s+\cdots+b_{i, j, \mathrm{nb}_{i, j}} s^{\mathrm{nb}_{\mathrm{i}, j}} \\
& a_{i, j}(s)=a_{i, j, 1} s+\cdots+a_{i, j, \mathrm{na}_{i, j}} s^{\mathrm{na}_{i, j}} \\
& \theta_{i, j}-\quad \text { tempo morto } \\
& n b_{i, j}-\quad \text { ordem do polinômio } b_{i, j}(s) \\
& n a_{i, j}-\quad \text { ordem do polinômio } a_{i, j}(s)
\end{aligned}
$$

Os parâmetros relativos aos polinômios $a_{i, j}(s)$ e $b_{i, j}(s)$ podem ser agrupados. Desta forma, podemos representar os parâmetros do modelo da seguinte forma:

$$
\begin{aligned}
& a_{\mathrm{i}, \mathrm{j}}=\left\lfloor\begin{array}{llll}
a_{\mathrm{i}, \mathrm{j}, 1} & a_{\mathrm{i}, \mathrm{j}, 2} & \cdots & a_{\mathrm{i}, \mathrm{j}, \mathrm{na}_{\mathrm{i}, \mathrm{j}}}
\end{array}\right\rfloor \\
& b_{\mathrm{i}, \mathrm{j}}=\left[\begin{array}{llll}
b_{\mathrm{i}, \mathrm{j}, 0} & b_{\mathrm{i}, \mathrm{j}, 1} & \cdots & b_{\mathrm{i}, \mathrm{j}, \mathrm{nb}} \mathrm{b}_{\mathrm{i}, \mathrm{j}}
\end{array}\right\rfloor
\end{aligned}
$$

O sistema na forma multivariável, tem genericamente ny variáveis controladas e $n u$ variáveis manipuladas e o modelo do sistema completo pode ser representado pela seguinte matriz de transferência:

$$
? y(s)=G(s) ? u(s)
$$

sendo:

$$
\begin{aligned}
& ? y(s)=\left\lfloor ? y_{1}(s) \cdots ? y_{n y}(s)\right]^{T} \\
& ? u(s)=\left[? u_{1}(s) \cdots ? u_{n u}(s)\right]^{T} \\
& G(s)=\left[\begin{array}{ccc}
G_{1,1}(s) & \cdots & G_{1, n u}(s) \\
\vdots & & \\
G_{n y, 1}(s) & \cdots & G_{n y, n u}(s)
\end{array}\right]
\end{aligned}
$$

No caso multivariável, é conveniente agrupar todos os parâmetros do modelo da seguinte forma:

$$
a(\mathrm{~s})=\left[\begin{array}{ccc}
a_{1,1}(\mathrm{~s}) & \cdots & a_{1, \mathrm{nu}}(\mathrm{s}) \\
\vdots & & \\
a_{\mathrm{ny}, 1}(\mathrm{~s}) & \cdots & a_{\mathrm{ny}, \mathrm{nu}}(\mathrm{s})
\end{array}\right] ; \quad b(\mathrm{~s})=\left[\begin{array}{ccc}
b_{1,1}(\mathrm{~s}) & \cdots & b_{1, \mathrm{nu}}(\mathrm{s}) \\
\vdots & & \\
b_{\mathrm{ny}, 1}(\mathrm{~s}) & \cdots & b_{\mathrm{ny}, \mathrm{nu}}(\mathrm{s})
\end{array}\right]
$$




$$
\theta(s)=\left[\begin{array}{ccc}
\theta_{1,1}(s) & \cdots & \theta_{1, n u}(s) \\
\vdots & & \\
\theta_{n y, 1}(s) & \cdots & \theta_{n y, n u}(s)
\end{array}\right]
$$

Os algoritmos de controle aqui utilizados, usam internamente modelos de resposta ao degrau em malha aberta. Portanto, aplicando um degrau unitário em $\Delta \boldsymbol{u}(\boldsymbol{s})$, para cada par $\boldsymbol{i j}$, temos:

$$
\Delta y_{d_{i, j}}(s)=G_{i, j}(s) \Delta u_{j}(s)
$$

sendo:

$$
\Delta u_{j}(s)=\frac{1}{s}
$$

Na forma explícita, temos a seguinte função correspondente à resposta ao degrau unitário:

$$
\Delta y_{d_{i, j}}(s)=\frac{b_{i, j, 0}+b_{i, j, 1} s+\cdots+b_{i, j, n b} s^{n b}}{s\left(1+a_{i, j, 1} s+\cdots+a_{i, j, n a} s^{n a}\right)} e^{-s \theta_{i, j}}
$$

Assumindo que o denominador não tenha raízes múltiplas, o que é razoável, pois em processos químicos dificilmente temos sistemas com constantes de tempo repetidas. Por conveniência, as raízes de $1+a_{i, j}(s)$ são representadas como:

$$
r_{i, j}=\left[\begin{array}{lll}
r_{i, j, 1} & \cdots & r_{i, j, n a}
\end{array}\right]^{T} \quad r_{i, j} \in \mathrm{C}^{n a \times 1}
$$

A resposta ao degrau do sistema pode então ser fatorada, obtendo-se a seguinte expressão:

$$
y_{d_{i, j}}(s)=\frac{b_{i, j}(\mathrm{~s})}{s\left(s-r_{i, j, 1}\right)\left(s-r_{i, j, 2}\right) \cdots\left(s-r_{i, j, n a}\right) \mathrm{s}} e^{-\mathrm{s} ?_{i, j}}
$$


O termo repetido em " $\mathrm{s}$ " indica a presença de características integradoras no processo.

Separando a resposta ao degrau unitário em frações parciais, temos:

$$
y_{d_{i, j}}(s)=\frac{c_{i, j, 0}}{s} e^{-s \theta_{i, j}}+\frac{c_{i, j, 1}}{s-r_{i, j, 1}} e^{-s \theta_{i, j}}+\cdots+\frac{c_{i, j, n a-1}}{s-r_{i, j, n a-1}} e^{-s \theta_{i, j}}+\frac{c_{i, j, n a}}{s^{2}} e^{-s \theta_{i, j}}
$$

sendo:

$$
c_{\mathrm{i}, \mathrm{j}, \mathrm{g}}=\lim _{s \rightarrow r_{\mathrm{i}, \mathrm{j}, \mathrm{g}}}\left(s-r_{\mathrm{i}, \mathrm{j}, \mathrm{g}}\right) \frac{b_{\mathrm{i}, \mathrm{j}}(s)}{s\left(s-r_{\mathrm{i}, \mathrm{j}, 1}\right)\left(s-r_{\mathrm{i}, \mathrm{j}, 2}\right) \cdots\left(s-r_{\mathrm{i}, \mathrm{j}, \mathrm{na}}\right)}
$$

Transformando a expressão da resposta ao degrau para o domínio do tempo temos a seguinte forma geral:

$$
y_{d_{i, j}}(t)=c_{i, j, 0}+c_{i, j, 1} e^{-r_{i, j, 1}\left(t-\theta_{i, j}\right)}+\cdots+c_{i, j, n a-1} e^{-r_{i, j, n a-1}\left(t-\theta_{i, j}\right)}+c_{i, j, n a}\left(t-\theta_{i, j}\right)
$$

Simplificando a expressão (8.18), temos:

$$
y_{d_{i, j}}(t)=\sum_{g=0}^{n a-1} c_{i, j, g} e^{-r_{i, j, g}\left(t-\theta_{i, j}\right)}+c_{i, j, n a}\left(t-\theta_{i, j}\right)
$$

Os coeficientes da resposta ao degrau do sistema podem ser obtidos a partir da eq. (8.19):

$$
\begin{aligned}
& s_{i, j, n}=0 \forall n T \leq \theta_{i, j} \\
& \left.s_{i, j, n}=c_{i, j, 0}+\sum_{g=1}^{n a} c_{i, j, g} f_{i, j, g}(n T) \quad \forall n T\right\rangle \theta_{i, j}
\end{aligned}
$$

sendo:

$$
\begin{aligned}
& n \quad \text { - instante de amostragem } \\
& f_{i, j, 0}(t)=1 \\
& f_{i, j, g}(t)=e^{-r_{i, j, g}\left(t-\theta_{i, j}\right)} \forall r_{i, j, g} \neq 0 \\
& f_{i, j, g}(t)=t-\theta_{i, j} \forall r_{i, j, g}=0
\end{aligned}
$$


Para o sistema multivariável é conveniente agrupar os parâmetros do modelo da seguinte forma:

$$
\begin{aligned}
& C_{0}=\left[\begin{array}{ccc}
c_{1,1,0} & \cdots & c_{1, n u, 0} \\
\vdots & & \vdots \\
c_{n y, 1,0} & \cdots & c_{n y, n u, 0}
\end{array}\right] \quad C_{0} \in \mathfrak{R}^{n y \times n u} \\
& C=\left[\begin{array}{ccc}
c_{1,1} & \cdots & c_{1, n u} \\
\vdots & & \vdots \\
c_{n y, 1} & \cdots & c_{n y, n u}
\end{array}\right] \quad C \in \mathrm{C}^{n y \times n u . n a} \\
& c_{i, j}=\left[\begin{array}{llll}
c_{i, j, 1} & c_{i, j, 2} & \ldots & c_{i, j, n a}
\end{array}\right] \\
& r_{0}=\left[\begin{array}{ccc}
0 & \cdots & 0 \\
\vdots & & \vdots \\
0 & \cdots & 0
\end{array}\right] \quad r_{0} \in \Re^{n y \times n u} \\
& r=\left[\begin{array}{ccc}
r_{1,1} & \cdots & r_{1, n u} \\
\vdots & & \vdots \\
r_{n y, 1} & \cdots & r_{n y, n u}
\end{array}\right] \quad r \in \mathrm{C}^{n y \times n u . n a} \\
& r_{i, j}=\left[\begin{array}{llll}
r_{i, j, 1} & r_{i, j, 2} & \ldots & r_{i, j, n a}
\end{array}\right]
\end{aligned}
$$

A resposta ao degrau é representada por:

$$
S_{n}=\left[\begin{array}{ccc}
S_{1,1, n} & \cdots & S_{1, n u, n} \\
\vdots & & \vdots \\
S_{n y, 1, n} & \cdots & s_{n y, n u, n}
\end{array}\right] \quad S_{n} \in \mathfrak{R}^{n y \times n u}
$$

\subsection{2 - Predição com modelos paramétricos}

Para um processo qualquer, podemos representar a predição da variável controlada da seguinte forma:

$$
\left[y_{i, k+n}\right]_{k / k-1}=\left[y_{i, k+n}\right]_{k-1 / k-1}+\sum_{j=1}^{n u} s_{i, j, n} \Delta u_{j, k-1}
$$


Podemos substituir $\boldsymbol{s}_{i, j, n}$ da eq. (8.20) na equação (8.1), obtendo:

$$
\left[y_{i, k+n}\right]_{k / k-1}=\left[y_{i, k+n}\right]_{k-1 / k-1}+\sum_{j=1}^{n u} \sum_{g=0}^{n a} c_{i, j, g} f_{i, j, g}(n T) \Delta u_{j, k-1}
$$

Separando os termos independentes dos dependentes do tempo, temos:

$$
\left[y_{i, k+n}\right]_{k / k-1}=\left[y_{i, k+n}\right]_{k-1 / k-1}+\sum_{j=1}^{n u} c_{i, j, 0} \Delta u_{j, k-1}+\sum_{j=1}^{n u} \sum_{g=1}^{n a} c_{i, j, g} f_{i, j, g}(n T) \Delta u_{j, k-1}
$$

A equação (8.25) nos induz a supor que a equação da predição pode ser reescrita da seguinte forma:

$$
\begin{array}{r}
{\left[y_{i, k+n}\right]_{k / k-1}=\left[p_{i}\right]_{k-1 / k-1}+\sum_{j=1}^{n u} \sum_{g=1}^{n a}\left[p_{i, j, g}\right]_{k-1 / k-1} f_{i, j, g}(n T)} \\
\forall n \mid n T>\max _{i} \theta_{i j}
\end{array}
$$

sendo:

$$
\left[p_{i}\right]_{k-1 / k-1} e\left[p_{i, j, g}\right]_{k-1 / k-1} \text { os parâmetros dessa nova representação. }
$$

Considerando apenas sistemas não integradores e usando a definição de $f_{i, j, g}$ em (8.26), temos:

$$
\left[y_{i, k+n}\right]_{k / k-1}=\left[y_{i, k+n+1}\right]_{k-1 / k-1}+\sum_{j=1}^{n u} c_{i, j, 0} \Delta u_{j, k-1}+\sum_{j=1}^{n u} \sum_{g=1}^{n a} c_{i, j, g} e^{r_{i, j, g} T} f_{i, j, g}(n T) \Delta u_{j, k-1}
$$

como:

$$
\left[y_{i, k+n+1}\right]_{k-1 / k-1}=\left[p_{i}\right]_{k-1 / k-1}+\sum_{j=1}^{n u} \sum_{g=1}^{n a}\left[p_{i, j, g}\right]_{k-1 / k-1} f_{i, j, g}((n+1) T)
$$

$\mathrm{ou}$ 
Capítulo 8 - Algoritmos de controle

80

$$
\left[y_{i, k+n+1}\right]_{k-1 / k-1}=\left[p_{i}\right]_{k-1 / k-1}+\sum_{j=1}^{n u} \sum_{g=1}^{n a}\left[p_{i, j, g}\right]_{k-1 / k-1} e^{r_{i, j, g} T} f_{i, j, g}(n T)
$$

substituindo (8.28) na equação (8.27), temos:

$$
\begin{aligned}
& {\left[y_{i, k+n}\right]_{k / k-1}=\left[p_{i}\right]_{k-1 / k-1}+\sum_{j=1}^{n u} c_{i, j, 0} \Delta u_{j, k-1}+} \\
& +\sum_{j=1}^{n u} \sum_{g=1}^{n a}\left(\left[p_{i, j, g}\right]_{k-1 / k-1} e^{r_{i, j, g} T}+c_{i, j, g} e^{r_{i, j, g} T} \Delta u_{j, k-1}\right) f_{i, j, g}(n T)
\end{aligned}
$$

Daí a eq. (8.29) pode ser reescrita como:

$$
\left[y_{i, k+n}\right]_{k / k-1}=\left[p_{i}\right]_{k / k-1}+\sum_{j=1}^{n u} \sum_{g=1}^{n a}\left[p_{i, j, g}\right]_{k / k-1} f_{i, j, g}(n T)
$$

sendo:

$$
\begin{aligned}
& {\left[p_{i}\right]_{k / k-1}=\left[p_{i}\right]_{k-1 / k-1}+\sum_{j=1}^{n u} c_{i, j, 0} \Delta u_{j, k-1}} \\
& {\left[p_{i, j, g}\right]_{k / k-1}=\left[p_{i, j, g}\right]_{k-1 / k-1} e^{r_{i, j, g} T}+c_{i, j, g} e^{r_{i, j, g} T} \Delta u_{j, k-1}}
\end{aligned}
$$

fazendo $d_{i, j, g}=e^{r_{i, j, g} T}$, temos:

$$
\left\lfloor p_{i, j, g}\right\rfloor_{k / k-1}=d_{i, j, g}\left\lfloor p_{i, j, g}\right\rfloor_{k-1 / k-1}+c_{i, j, g} d_{i, j, g} \Delta u_{j, k-1}
$$

As equações (8.30) e (8.31) são as equações que atualizam os parâmetros da equação (8.26), que foi proposta para a predição das variáveis controladas.

Vamos procurar agora uma forma vetorial para a equação (8.26), que pode ser escrita como:

$$
\begin{gathered}
{\left[y_{i, k+n}\right]_{k / k-1}=\left[p_{i}\right]_{k / k-1}+\sum_{j=1}^{n u}\left\{\left[p_{i, j, 1}\right]_{k / k-1} f_{i, j, 1}(n T)+\cdots+\left[p_{i, j, n a}\right]_{k / k-1} f_{i, j, n a}(n T)\right\}} \\
\left\lfloor y_{i, k+n}\right\rfloor_{k / k-1}=\left[p_{i}\right]_{k / k-1}+\left\lfloor p_{i, 1,1}\right\rfloor_{k / k-1} f_{i, 1,1}(n T)+\cdots+\left\lfloor p_{i, 1, n a}\right\rfloor_{k / k-1} f_{i, 1, n a}(n T)+\cdots+ \\
\left.+\left[p_{i, n u, 1}\right]_{k / k-1} f_{i, n u, 1}(n T)+\cdots+\left[p_{i, n u, n a}\right]_{k / k-1} f_{i, n u, n a}(n T)\right\}
\end{gathered}
$$


Escrevendo a eq. (8.32) para $i=1,2, \ldots, n y$, temos:

$$
\begin{aligned}
& {\left[y_{1, k+n}\right]_{\mathrm{k} / \mathrm{k}-1}=\left[p_{1}\right]_{\mathrm{k} / \mathrm{k}-1}+\left\{\left[p_{1,1,1}\right]_{\mathrm{k} / \mathrm{k}-1} f_{1,1,1}(n T)+\cdots+\left[p_{1,1, n a}\right]_{\mathrm{k} / \mathrm{k}-1} f_{1,1, n a}(n T)+\cdots+\right.} \\
& \left.+\left[p_{1, n u, 1}\right]_{\mathrm{k} / \mathrm{k}-1} f_{1, n u, 1}(n T)+\cdots+\left[p_{1, n u, n a}\right]_{\mathrm{k} / \mathrm{k}-1} f_{1, n u, n a}(n T)\right\} \\
& \vdots \\
& {\left[y_{n y, k+n}\right]_{\mathrm{k} / \mathrm{k}-1}=\left[p_{n y}\right]_{\mathrm{k} / \mathrm{k}-1}+\left\{\left[p_{n y, l, 1}\right]_{\mathrm{k} / \mathrm{k}-1} f_{n y, 1,1}(n T)+\cdots+\left[p_{n y, 1, n a}\right]_{\mathrm{k} / \mathrm{k}-1} f_{n y, l, n a}(n T)+\cdots+\right.} \\
& \left.+\left[p_{n y, n u, 1}\right]_{\mathrm{k} / \mathrm{k}-1} f_{n y, n u, 1}(n T)+\cdots+\left[p_{n y, n u, n a}\right]_{\mathrm{k} / \mathrm{k}-1} f_{n y, n u, n a}(n T)\right\}
\end{aligned}
$$

Vetorialmente temos:

$$
\left[y_{k+n}\right]_{k / k-1}=\left[p^{i}\right]_{k / k-1}+\psi_{n}\left[p^{t}\right]_{k / k-1}
$$

sendo:

$$
\begin{aligned}
& {\left[y_{k+n}\right]_{k / k-1}=\left[\left[y_{1, k+n}\right]_{k / k-1} \cdots\left[y_{n y, k+n}\right]_{k / k-1}\right]^{T} \quad\left[y_{k+n}\right]_{k / k-1} \in \mathfrak{R}^{n y \times 1}} \\
& {\left[p^{i}\right]_{k / k-1}=\left[\left[p_{1}\right]_{k / k-1} \cdots\left[p_{n y}\right]_{k / k-1}\right]^{T} \quad\left[p^{i}\right]_{k / k-1} \in \mathfrak{R}^{n y \times 1}} \\
& {\left[p^{t}\right]_{k / k-1}=\left[\left[p_{1,1,1}\right]_{k / k-1} \cdots\left[p_{1,1, n a}\right]_{k / k-1} \cdots\left[p_{1, n u, 1}\right]_{k / k-1} \cdots\left[p_{1, n u, n a}\right]_{k / k-1} \cdots\right.} \\
& \left.\left[p_{n y, 1,1}\right]_{k / k-1} \cdots\left[p_{n y, 1, n a}\right]_{k / k-1} \cdots\left[p_{n y, n u, 1}\right]_{k / k-1} \cdots\left[p_{n y, n u, n a}\right]_{k / k-1}\right]^{T} \quad\left[p^{t}\right]_{k / k-1} \in \mathfrak{R}^{n y . n u . n a \times 1} \\
& \Psi_{n}=\left[\begin{array}{cccc}
f_{1}(n) & 0 & \cdots & 0 \\
0 & f_{2}(n) & \cdots & 0 \\
\vdots & \vdots & \ddots & \vdots \\
0 & 0 & \cdots & f_{n y}(n)
\end{array}\right] ; \quad \Psi_{n} \in \mathfrak{R}^{n y \times n y . n u, n a}
\end{aligned}
$$

sendo

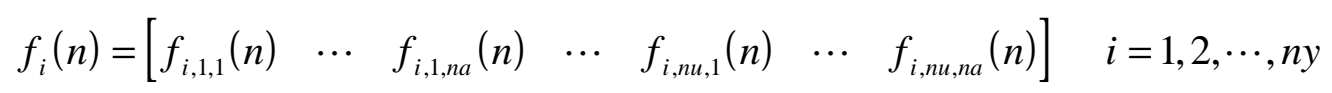

Escrevendo a eq. (8.30) para $i=1,2, \ldots, n y$, temos: 


$$
\left[p^{i}\right]_{k / k-1}=\left[p^{i}\right]_{k-1 / k-1}+C_{o} \Delta u_{k-1}
$$

Analogamente, a eq. (8.31) para $i=1,2, \ldots, n y$, leva à seguinte relação:

$$
\left[p^{t}\right]_{k / k-1}=D\left[p^{t}\right]_{k-1 / k-1}+C D N_{p} \Delta u_{k-1}
$$

sendo:

$$
\begin{aligned}
& D=\left(d_{1,1,1} \cdots d_{1,1, n a} \cdots d_{1, n u, 1} \cdots d_{1, n u, n a} \cdots d_{n y, 1,1} \cdots d_{n y, 1, n a} \cdots d_{n y, n u, 1} \cdots d_{n y, n u, n a}\right) \\
& D \in \Re^{\text {ny.nu.na } \times n y . n u . n a} \\
& C=\left(c_{1,1,1} \cdots c_{1,1, n a} \cdots c_{1, n u, 1} \cdots c_{1, n u, n a} \cdots c_{n y, 1,1} \cdots c_{n y, 1, n a} \cdots c_{n y, n u, 1} \cdots c_{n y, n u, n a}\right) \\
& C \in C^{n y . n u . n a \times n y . n u . n a ~} \\
& N_{p}=\left[\begin{array}{c}
J_{1} \\
\vdots \\
J_{n y}
\end{array}\right] \quad J_{i}=\left[\begin{array}{cccc}
{\left[\begin{array}{cccc}
1 & 0 & \cdots & 0 \\
\vdots & \vdots & & \vdots \\
1 & 0 & \cdots & 0
\end{array}\right]} \\
{\left[\begin{array}{cccc}
0 & 1 & \cdots & 0 \\
\vdots & \vdots & & \vdots \\
0 & 1 & \cdots & 0
\end{array}\right]} \\
{\left[\begin{array}{cccc}
0 & 0 & \cdots & 1 \\
\vdots & \vdots & & \vdots \\
0 & 0 & \cdots & 1
\end{array}\right]}
\end{array}\right] \quad J_{i} \in \mathfrak{R}^{\text {nu.na } \times n u}
\end{aligned}
$$

\subsection{3 - Cálculo das predições das variáveis controladas}

Para que a ordem do modelo seja reduzida é necessário que se reduza o número de estados utilizados para o cálculo das predições das variáveis controladas.

Para modelos paramétricos, o vetor de predições tem duas partes com características diferentes. A primeira parte é idêntica ao caso em que se usa modelos de convolução (item 8.1) e a segunda parte que utiliza o modelo paramétrico em instantes 
previamente definidos, que normalmente estão distribuídos em instantes de tempo esparsos.

Uma vantagem direta desta abordagem é a redução substancial das dimensões do vetor da predição e da matriz de transição de estado $(\mathrm{M})$, onde, também podemos incluir instantes bem distantes e as predições no estado estacionário.

As mesmas etapas utilizadas para o cálculo das predições com modelos não paramétricos, feitas no parágrafo 8.1.1, são utilizadas no cálculo das predições com modelos paramétricos. Nos próximos itens apresentaremos as alterações feitas nos cálculos das predições com a utilização de modelos de ordem reduzida.

\subsubsection{1 - Efeito da última ação de controle}

O vetor das predições das variáveis controladas é composto de duas partes:

1) Parte referente ao tempo morto, onde a variável controlada não sofre o efeito do $\Delta u_{k-1}$, ou instantes em que se deseja intervalos de tempo sucessivos acima do tempo morto, ou seja:

$$
s_{i, j, n}=0 \quad \forall n T \leq \theta_{i, j}
$$

A parte do vetor de predições das saídas, dentro do tempo morto, tem a mesma estrutura que o caso convencional apresentada em (8.1).

2) Parte onde $\Delta u_{k-1}$ afeta os valores das predições, que pode ter uma estrutura diferente, por exemplo, considerando instantes de tempo esparsos.

Na definição dos instantes das predições deve-se considerar a existência dessas 2 partes. Assim, os instantes até o maior tempo morto mais um período de amostragem devem ser seqüenciais, ou seja:

$$
n_{0}=\max _{i, j}\left\{\operatorname{int}\left(\theta_{i, j} / T\right)\right\}
$$


Após o instante $\boldsymbol{n}_{\boldsymbol{0}}$, as predições podem ser calculadas em instantes esparsos. Entretanto, em todos os instantes desejados, elas podem ser corrigidas com a última ação de controle da seguinte forma:

$$
[y]_{k / k-1}=[y]_{k-1 / k-1}+S \Delta u_{k-1}
$$

sendo:

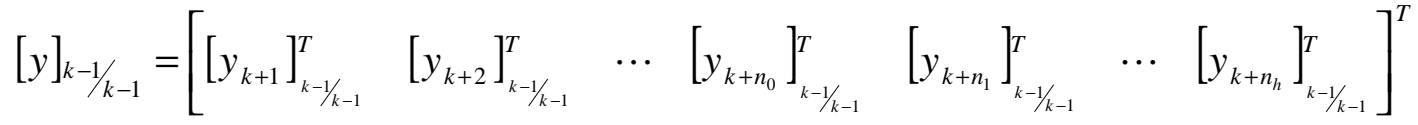

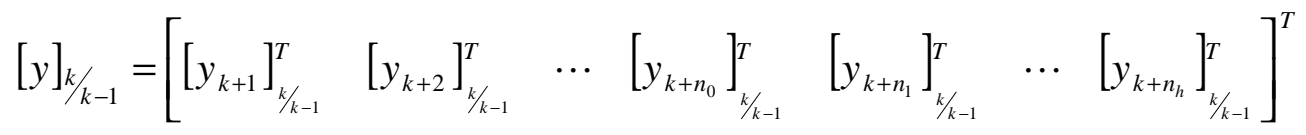

$$
\begin{aligned}
& \Delta u_{k-1}=\left[\Delta u_{1, k-1} \cdots \Delta u_{n u, k-1}\right]^{T} \\
& S=\left[\begin{array}{lllllll}
S_{1}^{T} & S_{2}^{T} & \cdots & S_{n 0}^{T} & S_{n_{1}}^{T} & \cdots & S_{n_{h}}^{T}
\end{array}\right]^{T}
\end{aligned}
$$

sendo:

np - número de instantes de predição

$$
n p=n_{0}+h
$$

$n_{0}$ - números de instantes seqüenciais

$h$ - número de instantes esparsos

\subsubsection{2 - Correção das predições com as leituras da planta}

Com o efeito da última ação de controle calculado, devemos corrigir as predições através da leitura das variáveis controladas. Para o cálculo dessa correção, utilizamos apenas os termos correspondentes ao instante $\boldsymbol{k}+\boldsymbol{l}$ do vetor de predições da mesma forma da abordagem utilizada na predição com modelos não paramétricos, porém as dimensões da matriz $\mathrm{N}$ são diferentes, ou seja:

$$
\left[y_{k+1}\right]_{k / k-1}=N[y]_{k / k-1}
$$


sendo:

$$
N=\left[\begin{array}{ccccccc}
1 & 0 & \cdots & 0 & 0 & \cdots & 0 \\
0 & 1 & \cdots & 0 & 0 & \cdots & 0 \\
\vdots & \vdots & \ddots & \vdots & \vdots & & \vdots \\
0 & 0 & \cdots & 1 & 0 & \cdots & 0
\end{array}\right] \quad ; \quad N \in \Re^{n y \times n y(n p+1+n u . n a)}
$$

Para as predições através de modelos paramétricos, as correções das predições com as leituras da planta são efetuadas da seguinte forma:

$$
\left[y_{i, k+n}^{c}\right]_{k / k-1}=\left[p_{i}\right]_{k / k-1}+\sum_{j=1}^{n u} \sum_{g=1}^{n a}\left[p_{i, j, g}\right]_{k / k-1} f_{i, j, g}(n T)+w_{i, k}
$$

como $\boldsymbol{w}_{i, k}$ não é função do tempo, a correção só é aplicada ao valor de $\left[p_{i}\right]_{k / k-1}$.

Portanto, a correção dos parâmetros fica da seguinte forma:

$$
\begin{aligned}
& {\left[p_{i}^{c}\right]_{k / k-1}=\left[p_{i}\right]_{k-1 / k-1}+\sum_{j=1}^{n u} c_{i, j, 0} ? u_{j, k-1}+w_{i, k}} \\
& {\left[p_{i, j, g}^{c}\right]_{k / k-1}=\left[p_{i, j, g}\right]_{k-1 / k-1} e^{r_{i, j, g} T}+c_{i, j, g} e^{r_{i, j, g} T} ? u_{j, k-1}}
\end{aligned}
$$

Como no controlador convencional, podemos corrigir o desvio da predição em relação à leitura da planta utilizando uma matriz $\mathrm{K}$ com dimensões relativas ao novo vetor de predições, portanto a nova correção é apresentada como:

$$
\left[y^{c}\right]_{k / k-1}=[y]_{k / k-1}+K W_{k}
$$

sendo: 


$$
K=\left[\begin{array}{cccc}
1 & 0 & \cdots & 0 \\
0 & 1 & \cdots & 0 \\
\vdots & \vdots & \ddots & \vdots \\
0 & 0 & \cdots & 1 \\
1 & 0 & \cdots & 0 \\
\vdots & \vdots & & \vdots \\
0 & 0 & \cdots & 1 \\
\vdots & \vdots & & \vdots \\
1 & 0 & \cdots & 0 \\
\vdots & \vdots & & \vdots \\
0 & 0 & \cdots & 1 \\
\vdots & \vdots & & \vdots \\
0 & 0 & \cdots & 0 \\
\vdots & \vdots & & \vdots \\
0 & 0 & \cdots & 0
\end{array}\right]
$$

\subsubsection{3 - Translação da predição corrigida}

As predições das variáveis controladas estão atualizadas e corrigidas para o instante $\boldsymbol{k}$. Isto significa que:

$$
\begin{aligned}
& {\left[y_{i, k+2}^{c}\right]_{k / k-1} \text { - corresponde ao instante atual } \boldsymbol{k + 1}} \\
& {\left[y_{i, k+3}^{c}\right]_{k / k-1} \text { - corresponde ao instante futuro } \boldsymbol{k}+\boldsymbol{2}} \\
& \vdots \\
& {\left[y_{i, k+n_{k}+1}^{c}\right]_{k / k-1} \text { - corresponde ao instante futuro } \boldsymbol{k}+\boldsymbol{n}_{\boldsymbol{k}}}
\end{aligned}
$$

Portanto, dentro da filosofia dos controladores preditivos, que apresentam um horizonte móvel, é necessário fazer a translação de um instante de tempo em todo vetor de predições e para todas as variáveis controladas. 


$$
\begin{gathered}
{\left[y_{1, k+1}\right]_{k / k}=\left[y_{1, k+2}^{c}\right]_{k / k-1}} \\
\vdots \\
{\left[y_{n y, k+1}\right]_{k}=\left[y_{n y, k+2}^{c}\right]_{k} / k-1} \\
{\left[y_{1, k+2}\right]_{k / k}=\left[y_{1, k+3}^{c}\right]_{k / k-1}} \\
\vdots \\
{\left[y_{n y, k+2}\right]_{k / k}=\left[y_{n y, k+3}^{c}\right]_{k / k-1}} \\
\vdots \\
{\left[y_{1, k+n_{k}}\right]_{k / k}=\left[y_{1, k+n_{k}+1}^{c}\right]_{k / k-1}} \\
\vdots \\
{\left[y_{n y, k+n}\right]_{k / k}=\left[y_{n y, k+n+1}^{c}\right]_{k / k-1}}
\end{gathered}
$$$$
\text { para } \boldsymbol{n} \leq \boldsymbol{n}_{\boldsymbol{o}}
$$$$
\text { sendo: } \boldsymbol{n}_{\boldsymbol{o}} \geq \operatorname{máx} \theta_{\mathrm{i}, \mathrm{j}}
$$

Para $\boldsymbol{n}>\boldsymbol{n}_{\boldsymbol{o}}$ usamos a eq. (8.33), assim temos:

$$
\left.\left[y_{k+n+1}\right]_{k / k}=\left\lfloor p^{i}\right\rfloor_{k / k}+\psi_{\mathrm{n}+1} \mid p^{t}\right\rfloor_{k / k}
$$

Para os instantes esparsos temos:

$$
\begin{aligned}
& {\left[y_{k+n_{1}}\right]_{k / k}=\left[p^{i}\right]_{k / k}+\psi_{\mathrm{n}_{1}+1}\left[p^{t}\right]_{k / k}} \\
& {\left[y_{k+n_{2}}\right]_{k / k}=\left[p^{i}\right]_{k / k}+\psi_{\mathrm{n}_{2}+1}\left[p^{t}\right]_{k / k}} \\
& \vdots \\
& {\left[y_{k+n_{k}}\right]_{k / k}=\left[p^{i}\right]_{k / k}+\psi_{\mathrm{n}_{\mathrm{k}}+1}\left[p^{t}\right]_{k / k}}
\end{aligned}
$$

Para os parâmetros da predição não temos a operação de translação, ou seja:

$$
\begin{aligned}
& {\left[p_{i, j, g}\right]_{k / k}=\left\lfloor p_{i, j, g}^{c}\right\rfloor_{k / k}} \\
& {\left[p_{i}\right]_{k / k}=\left\lfloor p_{i}^{c}\right]_{k / k} \quad \begin{array}{l}
i=1, \ldots, n y \\
j=1, \ldots, n u \\
g=1, \ldots, n a
\end{array}}
\end{aligned}
$$


Juntando as operações de translação, efeito da última ação de controle e correção da predição temos:

$$
[y]_{k / k}=M[y]_{k-1 / k-1}+S \Delta u_{k-1}+K W
$$

sendo:

$$
\begin{aligned}
& {[y]_{k / k}=\left[\begin{array}{lllllll}
{\left[y_{k+1}\right]_{k / k}} & {\left[y_{k+2}\right]_{k / k}} & \cdots & {\left[y_{k+n-1}\right]_{k / k}} & {\left[y_{k+n}\right]_{k / k}} & {\left[y_{k+n_{1}}\right]_{k / k}} & \cdots \\
\cdots & {\left[y_{k+n_{2}}\right]_{k / k}} & \cdots & {\left[y_{k+n_{h}}\right]_{k / k}} & {\left[p^{i}\right]_{k / k}} & {\left[p^{t}\right]_{k / k}} &
\end{array}\right]^{T}} \\
& {[y]_{k / k} \in \mathfrak{R}^{n y(n p+1+n u . n a) \times l}}
\end{aligned}
$$

sendo $n p=n_{0}+h$

$$
\begin{aligned}
& M=\left[\begin{array}{cccccccccccc}
0 & I_{n y} & 0 & \cdots & 0 & 0 & 0 & 0 & \cdots & 0 & 0 & 0 \\
0 & 0 & I_{n y} & \cdots & 0 & 0 & 0 & 0 & \cdots & 0 & 0 & 0 \\
\vdots & \vdots & \vdots & \ddots & \vdots & \vdots & \vdots & \vdots & \ddots & \vdots & \vdots & \vdots \\
0 & 0 & 0 & \cdots & I_{n y} & 0 & 0 & 0 & \cdots & 0 & 0 & 0 \\
0 & 0 & 0 & \cdots & 0 & 0 & 0 & 0 & \cdots & 0 & I_{n y} & \Psi_{n_{0}+1} \\
0 & 0 & 0 & \cdots & 0 & 0 & 0 & 0 & \cdots & 0 & I_{n y} & \Psi_{n_{1}+1} \\
0 & 0 & 0 & \cdots & 0 & 0 & 0 & 0 & \cdots & 0 & I_{n y} & \Psi_{n_{2}+1} \\
\vdots & \vdots & \vdots & \ddots & \vdots & \vdots & \vdots & \vdots & \ddots & \vdots & \vdots & \vdots \\
0 & 0 & 0 & \cdots & 0 & 0 & 0 & 0 & \cdots & 0 & I_{n y} & \Psi_{n_{h}+1} \\
0 & 0 & 0 & \cdots & 0 & 0 & 0 & 0 & \cdots & 0 & I_{n y} & 0 \\
0 & 0 & 0 & \cdots & 0 & 0 & 0 & 0 & \cdots & 0 & 0 & D
\end{array}\right] \\
& M \in \mathfrak{R}^{n y(n p+1+n u . n a) \times n y(n p+1+n u . n a)} \\
& S=\left[\begin{array}{lllllllllll}
S_{2}^{T} & S_{3}^{T} & \cdots & S_{n_{0}}^{T} & S_{n_{0}+1}^{T} & S_{n_{1}+1}^{T} & S_{n_{2}+1}^{T} & \cdots & S_{n_{k}+1}^{T} & C_{0}^{T} & C D N_{p}
\end{array}\right]^{T} \\
& S \in \mathfrak{R}^{n y(n p+1+n u . n a) \times n u}
\end{aligned}
$$

A matriz M fornece a translação para instantes de tempo seqüências e esparsos. 


\subsection{4 - Cálculo das ações de controle}

O algoritmo de controle preditivo com modelo paramétrico calcula as ações de controle da mesma forma descrita anteriormente, alterando apenas a construção das matrizes envolvidas. As etapas do algoritmo passam a ter a seguinte forma:

\subsubsection{1 - Trajetória referência das variáveis controladas}

Com a utilização dos modelos de estado (paramétrico), é necessário calcular as predições somente nos instantes em que o controlador irá minimizar o erro. Neste caso, o horizonte de predição (np) se confunde com o horizonte de otimização (nr).

Com as predições em tempos esparsos, os instantes de predição podem ser diferentes para cada uma das variáveis controladas. Isto é, se tivermos $\boldsymbol{k}$ instantes esparsos, o vetor de instantes esparsos para a variável $\boldsymbol{j}$ pode ser dado por:

$$
t_{j}=\left[\begin{array}{llll}
t_{j, 1} & t_{j, 2} & \cdots & t_{j, k}
\end{array}\right]
$$

A predição transferida de um instante de amostragem para outro é da forma:

$$
[y]_{k / k}=\left[\begin{array}{lllllllll}
y_{k+1} & y_{k+2} & \cdots & y_{k+n_{0}} & y_{k+n_{1}} & \cdots & y_{k+n h} & p^{i} & p^{t}
\end{array}\right]_{k / k}^{T}
$$

sendo:

$$
\begin{aligned}
& y_{k+i}=\left[\begin{array}{lll}
y_{1, k+i} & \cdots & y_{n y, k+i}
\end{array}\right]^{T} \\
& n_{0} \quad \text { - número de instantes de predição seqüenciais. } \\
& h \quad \text { - número de instantes esparsos das predições }\left(n p-n_{0}\right)
\end{aligned}
$$

As predições das variáveis controladas são alteradas pelas ações de controle em cada instante futuro. Desta forma, quando aplicamos “ $m$ ” ações de controle, temos: 


$$
\begin{aligned}
& {\left[\bar{y}_{k+1}\right]_{k / k}=\left[y_{k+1}\right]_{k / k}+S_{1} \Delta u_{k}} \\
& {\left[\bar{y}_{k+2}\right]_{k / k}=\left[y_{k+2}\right]_{k / k}+S_{2} \Delta u_{k}+S_{1} \Delta u_{k+1}} \\
& \vdots \\
& {\left[\bar{y}_{k+m}\right]_{k / k}=\left[y_{k+m}\right]_{k / k}+S_{m} \Delta u_{k}+S_{m-1} \Delta u_{k+1}+\cdots+S_{1} \Delta u_{k+m-1}} \\
& \vdots \\
& {\left[\bar{y}_{k+n k}\right]_{k / k}=\left[y_{k+n p}\right]_{k / k}+S_{n k} \Delta u_{k}+S_{n k-1} \Delta u_{k+1}+\cdots+S_{n k-m+1} \Delta u_{k+m-1}} \\
& {\left[\bar{p}^{i}\right]_{k / k}=\left[p^{i}\right]_{k / k}+C_{0} \Delta u_{k}+C_{0} \Delta u_{k+1}+\cdots+C_{0} \Delta u_{k+m-1}}
\end{aligned}
$$

Vetorialmente, temos:

$$
[\bar{y}]_{k / k}=[y]_{k / k}+S_{m} \Delta u
$$

sendo:

$$
\begin{aligned}
& {[\bar{y}]_{k / k}=\left[\begin{array}{lllllll}
\bar{y}_{k+1} & \bar{y}_{k+2} & \cdots & \bar{y}_{k+m} & \cdots & \bar{y}_{k+n k} & \bar{p}^{i}
\end{array}\right]_{k / k}^{T}} \\
& \Delta u=\left[\begin{array}{lll}
\Delta u_{1} & \cdots & \Delta u_{m}
\end{array}\right]^{T} \\
& S_{m}=\left[\begin{array}{cccc}
S_{1} & 0 & \cdots & 0 \\
S_{2} & S_{1} & \cdots & 0 \\
\vdots & \vdots & & \vdots \\
S_{m} & S_{m-1} & \cdots & S_{1} \\
\vdots & \vdots & & \vdots \\
S_{n k} & S_{n k-1} & \cdots & S_{n k-m+1} \\
C_{0} & C_{0} & \cdots & C_{0}
\end{array}\right]
\end{aligned}
$$

sendo:

$m$ - número de ações de controle futuras calculadas pelo controlador, conhecido como horizonte de controle

\subsubsection{2 - Cálculo dos erros das variáveis controladas}

Como visto para o MPC com modelo não paramétrico, no cálculo dos erros em relação aos respectivos setpoints, temos: 


$$
\left\lfloor e_{k+j}\right\rfloor_{k / k}=y_{s p}-\left\lfloor y_{k+j}\right\rfloor_{k / k}
$$

Na eq. (8.41), o vetor de predições inclui os parâmetros $\boldsymbol{p}^{i}$ e $\boldsymbol{p}^{t}$ da equação de predição (8.26). Assim, o vetor $\boldsymbol{y}_{\boldsymbol{s}}$ deve incluir os valores correspondentes aos setpoints desses parâmetros. Pela eq. (8.26), se o sistema for estável em malha aberta, então:

$$
\lim _{n \rightarrow \infty}\left[y_{k+n}\right]_{k / k-1}=\left[p^{i}\right]_{k-1 / k-1}
$$

Assim, para $\boldsymbol{p}^{i}$ o setpoint é o mesmo das variáveis controladas.

Resumindo, podemos representar o erro nas variáveis controladas e parâmetros como:

$$
[e]_{k / k}=N y_{s p}-[y]_{k / k}
$$

sendo:

$$
\begin{aligned}
& {[\mathrm{e}]_{\mathrm{k} / \mathrm{k}}=\left[\begin{array}{llllllll}
\mathrm{e}_{\mathrm{k}+1} & \mathrm{e}_{\mathrm{k}+2} & \cdots & \mathrm{e}_{\mathrm{k}+\mathrm{n}} & \mathrm{e}_{\mathrm{k}+\mathrm{n}_{1}} & \cdots & \mathrm{e}_{\mathrm{k}+\mathrm{n}_{\mathrm{k}}} & \mathrm{e}_{\mathrm{k}+\infty}
\end{array}\right]^{\mathrm{T}}} \\
& e_{k+\infty}=y_{s p}-\left\lfloor p^{i}\right\rfloor_{k / k} \\
& y_{s p}=\left[\begin{array}{lll}
y_{s p_{1}} & \cdots & y_{s p_{n y}}
\end{array}\right]^{T} \\
& {[y]_{k / k}=\left[\begin{array}{lllllllll}
y_{k+1} & y_{k+2} & \cdots & y_{k+n} & y_{k+n_{1}} & \cdots & y_{k+n_{k}} & p^{i} & p^{t}
\end{array}\right]^{T}}
\end{aligned}
$$




$$
N=\left[\begin{array}{ccc}
1 & \cdots & 0 \\
\vdots & \ddots & \vdots \\
0 & \cdots & 1 \\
1 & \cdots & 0 \\
\vdots & \ddots & \vdots \\
0 & \cdots & 1 \\
\vdots & & \vdots \\
1 & \cdots & 0 \\
\vdots & \ddots & \vdots \\
0 & \cdots & 1 \\
1 & \cdots & 0 \\
\vdots & \ddots & \vdots \\
0 & \cdots & 1 \\
0 & \cdots & 0 \\
\vdots & \ddots & \vdots \\
0 & \cdots & 0
\end{array}\right] \quad ; \quad \quad N \in \mathfrak{R}^{n y(n p+1+n u . n a) \times n y}
$$

\subsection{5 - Algoritmo de controle preditivo}

No caso do controlador preditivo multivariável (MPC) com modelos de ordem reduzida, temos para o erro após as ações a mesma expressão que foi usada no item 8.1.2.3:

$$
[\bar{e}]_{k / k}=[e]_{k / k}-S_{m} \Delta u
$$

porém os vetores $[\bar{e}]_{k / k}$, e $[e]_{k} / k$ e a matriz $S_{m}$ são definidas de forma diferente conforme apresentada na seção anterior.

Os algoritmos de controle apresentados anteriormente podem também utilizar essa nova formulação.

\subsubsection{1 - MMPC (Multi-Model Predictive Control)}


Descreveremos agora uma nova formulação do controlador preditivo onde vários modelos do processo são simultaneamente considerados. O problema de controle resultante é então resolvido usando a formulação da programação não-linear (NLP) utilizando o algoritmo tipo SQP (sequential quadratic programming) proposto por K. Schittkowski (1985).

Os controladores multi-modelos procuram resolver o problema de controle preditivo com sistemas variantes no tempo e no estado, isto é, têm uma faixa de operação mais ampla do que os controladores convencionais, em função das não-linearidades presentes no processo. O problema de controle é resolvido através da utilização de um conjunto de modelos, onde se descreve os diversos comportamentos do processo ao longo da região de operação do controlador.

\subsubsection{1 - Algoritmo MMPC}

O MMPC foi desenvolvido por Almeida Neto (1999) utilizando uma formulação baseada em LMI's (Linear Matrix Inequalities). Entretanto, não temos disponível nenhum pacote computacional para implementar essa solução em sistemas industriais. Assim, neste trabalho foi desenvolvida uma solução que possa ser implementada com os pacotes de programação não-linear disponíveis. Para tal considere a função objetivo do controlador representada pela equação (8.15):

$$
J_{k}=[e]_{k / k}^{T} \Gamma[e]_{k / k}-\Delta u^{T} S_{m}^{T} \Gamma[e]_{k / k}-[e]_{k / k}^{T} \Gamma S_{m} \Delta u+\Delta u^{T}\left(S_{m}^{T} \Gamma S_{m}+\Lambda\right) \Delta u
$$

Considerando que $\boldsymbol{J}_{\boldsymbol{k}}$ seja sempre menor do que um limite positivo $\gamma$, podemos representar o objetivo na forma:

$$
J_{k}\left\langle\gamma \quad \text { ou } \quad \gamma-J_{k}\right\rangle 0
$$

ou

$$
\gamma-[e]_{k / k}^{T} \Gamma[e]_{k / k}+\Delta u^{T} S_{m}^{T} \Gamma[e]_{k / k}+[e]_{k / k}^{T} \Gamma S_{m} \Delta u-\Delta u^{T}\left(S_{m}^{T} \Gamma S_{m}+\Lambda\right) \Delta u>0
$$


Dai, o problema que o MPC resolve pode ser colocado na forma:

\section{$\underset{\Delta u, \gamma}{\operatorname{Minimizar}} \gamma$}

$$
\begin{aligned}
\text { sujeito } \quad \text { a: } & \gamma-[e]_{k / k}^{T} \Gamma[e]_{k / k}+\Delta u^{T} S_{m}^{T} \Gamma[e]_{k / k}+[e]_{k / k}^{T} \Gamma S_{m} \Delta u-\Delta u^{T}\left(S_{m}^{T} \Gamma S_{m}+\Lambda\right) \Delta u>0 \\
& \gamma>0 \\
& \Delta u-\Delta u \min >0 \\
& -\Delta u+\Delta u \max >0
\end{aligned}
$$

que é um problema de otimização com função objetivo linear e restrições quadráticas, portanto um problema não-linear, que não pode ser resolvido com os pacotes computacionais de programação linear (LP) ou quadrática (QP).

\subsubsection{2 - Representação de incertezas do sistema}

Em geral, o modelo do processo varia ao longo do tempo, pois características como: vazão de carga e produtos, composição da carga e produtos, pressão de operação, sujeira de trocadores de calor variam no tempo. Estas incertezas podem ser incorporadas ao problema de controle através da utilização de vários modelos, sendo estes modelos colocados como novas restrições a serem obedecidas pelo controlador, estas novas restrições seriam da forma:

$$
\begin{aligned}
& \gamma-[e]_{k / k}^{T} \Gamma[e]_{k / k}+\Delta u^{T} S_{m, 1}^{T} \Gamma[e]_{k / k}+[e]_{k / k}^{T} \Gamma S_{m, 1} \Delta u-\Delta u^{T}\left(S_{m, 1}^{T} \Gamma S_{m, 1}+\Lambda\right) \Delta u>0 \\
& \gamma-[e]_{k / k}^{T} \Gamma[e]_{k / k}+\Delta u^{T} S_{m, 2}^{T} \Gamma[e]_{k / k}+[e]_{k / k}^{T} \Gamma S_{m, 2} \Delta u-\Delta u^{T}\left(S_{m, 2}^{T} \Gamma S_{m, 2}+\Lambda\right) \Delta u>0 \\
& \cdot \\
& \gamma-[e]_{k / k}^{T} \Gamma[e]_{k / k}+\Delta u^{T} S_{m, L}^{T} \Gamma[e]_{k / k}+[e]_{k / k}^{T} \Gamma S_{m, L} \Delta u-\Delta u^{T}\left(S_{m, L}^{T} \Gamma S_{m, L}+\Lambda\right) \Delta u>0
\end{aligned}
$$

onde $S_{m, 1}$. . . $S_{m, L}$ correspondem aos modelos obtidos em diferentes condições operacionais.

$L$-é o número de modelos alternativos do processo 
Nessa formulação, o problema do MMPC pode ser representado na seguinte forma:

$$
\begin{aligned}
& \text { Minimizar } \gamma \\
& \Delta u, \gamma \\
& \text { sujeito } \quad \text { a: } \quad \gamma-[e]_{k / k}^{T} \Gamma[e]_{k / k}+\Delta u^{T} S_{m}^{T} \Gamma[e]_{k / k}+[e]_{k / k}^{T} \Gamma S_{m} \Delta u-\Delta u^{T}\left(S_{m}^{T} \Gamma S_{m}+\Lambda\right) \Delta u>0 \\
& \gamma-[e]_{k / k}^{T} \Gamma[e]_{k / k}+\Delta u^{T} S_{m, 1}^{T} \Gamma[e]_{k / k}+[e]_{k / k}^{T} \Gamma S_{m, 1} \Delta u-\Delta u^{T}\left(S_{m, 1}^{T} \Gamma S_{m, 1}+\Lambda\right) \Delta u>0 \\
& \gamma-[e]_{k / k}^{T} \Gamma[e]_{k / k}+\Delta u^{T} S_{m, 2}^{T} \Gamma[e]_{k / k}+[e]_{k / k}^{T} \Gamma S_{m, 2} \Delta u-\Delta u^{T}\left(S_{m, 2}^{T} \Gamma S_{m, 2}+\Lambda\right) \Delta u>0 \\
& \gamma-[e]_{k / k}^{T} \Gamma[e]_{k / k}+\Delta u^{T} S_{m, L}^{T} \Gamma[e]_{k / k}+[e]_{k / k}^{T} \Gamma S_{m, L} \Delta u-\Delta u^{T}\left(S_{m, L}^{T} \Gamma S_{m, L}+\Lambda\right) \Delta u>0 \\
& \gamma>0 \\
& \Delta u-\Delta u_{\min }>0 \\
& -\Delta u+\Delta u \max >0
\end{aligned}
$$

Observe que o problema acima tem função objetivo linear e restrições quadráticas e portanto é um problema de NLP. Na planta industrial o controlador MMPC definido pelo problema é resolvido com um solver comercial baseado em SQP (K. Schittkowski , 1985). Neste trabalho não se buscou investigar qual seria o solver mais adequado para o problema do MMPC, que tem uma estrutura particular e talvez não a mais adequada para algoritmo tipo SQP. Em sistemas de grande porte essa consideração pode se tornar importante. 


\section{9}

\section{APLICAÇÃO DO MPC À COLUNA DEPROPANIZADORA}

O algoritmo DMC em sua formulação tradicional apresenta algumas limitações que devem ser consideradas durante o projeto de controle. As limitações básicas são:

$\checkmark$ Utilização de modelos de convolução discretos, que se constituem de uma seqüência de coeficientes que correspondem aos valores da resposta ao degrau unitário em instantes de amostragem sucessivos. Este fato faz com que o número de coeficientes da resposta ao degrau leve a uma limitação computacional devido à dimensão do problema, quando temos dinâmicas do processo muito lentas. Podemos contornar o problema utilizando algumas opções como: forçar o modelo a estabilizar no horizonte desejado, o que provoca um desvio em relação à planta; truncar o modelo do processo, tendo um modelo incompleto em relação à resposta da planta; ou aumentar o período de amostragem, podendo perder alguma informação importante entre duas amostragens sucessivas. Além disso fica muito difícil conciliar modelos com dinâmicas rápidas e lentas com o mesmo número de coeficientes da resposta ao degrau.

$\checkmark$ Com a limitação do número de coeficientes do modelo, a resposta ao degrau pode ser semelhante à de uma variável integradora quando a constante de tempo do processo for muito elevada. Isto faz com que o controlador tenha mais dificuldade em resolver o problema de controle, porque o controlador preditivo não lida com sistemas integradores diretamente.

$\checkmark$ Utilização de modelos lineares invariantes no tempo. Assim, não são adequados para resolver problemas de controle onde o processo tem comportamento não-linear ou onde o modelo do sistema se altera ao longo do tempo. O modelo utilizado é identificado em um ponto específico da região de operação do sistema. Daí o controlador é projetado para operar numa região onde o processo tem um dado comportamento. Isto resulta em incertezas nos ganhos e constantes de tempo do processo e conseqüentemente em perda de desempenho do controlador, quando operando em outras regiões. 
Para contornar estas limitações, neste trabalho modificamos o controlador convencional. As modificações testadas foram:

$\checkmark$ Uso do controlador em espaço de estado com modelos de resposta ao degrau analítico no domínio de tempo contínuo;

$\checkmark$ Utilização de um controlador com múltiplos modelos, formulando o problema de controle como um problema de Programação não-linear (MMPC).

Foram comparadas as performances de duas estratégias de controle. A primeira, que podemos chamar de convencional, foi representada pelo QDMC. A segunda, que podemos chamar de robusta, ou que pelo menos tenta enfocar o problema de estabilidade do sistema para incertezas no modelo, representada pelo MMPC proposto neste trabalho. Na segunda, foram utilizados os 5 modelos levantados no simulador e também o modelo levantado na planta industrial.

Os controladores foram avaliados através da observação da capacidade dos mesmos em manter o sistema em malha fechada estável, tanto para as variáveis controladas como as manipuladas, bem como a comparação das performances dos controladores, considerando os efeitos dos seus parâmetros de sintonia.

A avaliação da performance de um sistema de controle, pode ser feita através do estabelecimento de algum padrão de comportamento para o mesmo. Este padrão pode estar ligado ao estado estacionário do sistema, ao comportamento no regime transiente, à sensibilidade do sistema aos parâmetros do controlador e aos efeitos das nãolinearidades do sistema.

As avaliações das performances dos controladores realizadas neste trabalho foram feitas de forma qualitativa para o caso servo e regulador. Inicialmente foram comparadas as performances do QDMC e MMPC para alguns pontos de operação considerando os mesmos parâmetros de sintonia para os dois controladores. Após o ajuste da sintonia foram aplicados degraus nas variáveis controladas e perturbações, sendo verificada a estabilidade dos dois controladores. Em seguida, foi avaliada a performance de cada controlador quando sujeito a alterações no modelo da planta, mantendo-se a mesma sintonia do item anterior. Finalmente o controlador MMPC foi 
avaliado em operação na planta industrial, considerando-se a melhor sintonia obtida durante a simulação do controlador.

\section{1 - Comparação QDMC e MMPC}

Como já vimos, o controlador tem a seguinte configuração:

\section{Variáveis manipuladas:}

$\mathrm{MV}_{1}$ : vazão de refluxo de topo $\left(\boldsymbol{V}_{\boldsymbol{R} F}\right)$

$\mathrm{MV}_{2}$ : vazão de fluido de aquecimento $\left(\boldsymbol{V}_{\boldsymbol{G O P}}\right)$

Variáveis controladas:

$\mathrm{CV}_{1}: \mathrm{C}_{3}^{-}$do butano (\%vol.) $\quad\left(\boldsymbol{C}_{3}^{-}\right)$

$\mathrm{CV}_{2}$ : temperatura de topo da coluna $\left({ }^{\circ} \mathrm{C}\right) \quad\left(\boldsymbol{T}_{\text {topo }}\right)$

Variáveis perturbadoras:

$\mathrm{DV}_{1}$ : vazão de Carga $\left(\boldsymbol{V}_{\boldsymbol{F D}}\right)$

$\mathrm{DV}_{2}$ : temperatura do fluido de aquecimento $\quad\left(\boldsymbol{T}_{\boldsymbol{G O P}}\right)$

Os controladores QDMC E MMPC utilizados têm uma série de parâmetros que são comuns. Durante o estudo foi utilizado um período de amostragem de 1 minuto que é o valor desse parâmetro usado na unidade industrial. O horizonte de controle $(m)$ foi adotado igual a 2 em todas as simulações. No caso da utilização do MMPC o valor desse parâmetro deve ser o menor possível já que, como vimos no item 8.2.5.1.2, o problema de controle recai em um problema de NLP cujo tempo de computação depende do horizonte de controle. Entretanto, as conclusões não se alteram se um valor de $(m)$ maior for utilizado.

No ajuste do controlador, o modelo nominal utilizado na predição das variáveis controladas foi um modelo obtido através de uma combinação de todos os modelos. Essa combinação consistiu na aplicação da média aritmética para todos os parâmetros (ganho e dinâmica) para os modelos obtidos através do simulador e também aquele da planta industrial. Para o modelo da planta utilizamos o modelo da planta industrial. No cálculo da ação de controle utilizamos o "modelo combinado" para o algoritmo QDMC, e os modelos 1, 2, 3, 4, 5 para o algoritmo MMPC. Além disso foram utilizados fatores de importância $(\Gamma)$ de 1 tanto para o $\mathrm{C}_{3}{ }^{-}$no butano como para a Ttopo. $\mathrm{O}_{3}{ }^{-}$é controlado no "set-point" e a Ttopo numa faixa de $1^{\circ} \mathrm{C}$ entre os valores máximo e mínimo, já que sua especificação não é tão rigorosa, Almeida et al (1996) apresenta uma 
descrição de como implementar o controle por faixas. Os instantes de amostragem utilizados para o cálculo da predição das variáveis controladas foram 1, 2, 5, 7, 10, 15, $20,25,30,35,40,45,50,60,70$ para os dois controladores.

Nesta fase, comparamos a performance do MMPC e QDMC. Esta fase foi dividida em três etapas; sendo que na primeira fizemos o ajuste da sintonia do controlador (principalmente fator de supressão das manipuladas), na segunda etapa foi avaliada a performance dos controladores sujeitos a variações nas variáveis controladas e perturbações e por último os dois controladores foram avaliados quando sujeitos a alterações no modelo utilizado para a planta.

Na tabela 9.1, apresentamos os ganhos dos modelos utilizados nos controladores:

\begin{tabular}{|c|c|c|c|c|c|c|c|c|c|}
\hline Mdl & $\mathbf{V}_{\mathbf{R F}}$ & $\mathbf{V}_{\mathbf{G O P}}$ & $\mathbf{C}_{\mathbf{3}}^{-}$ & $\mathbf{T}_{\text {topo }}$ & $\begin{array}{c}\mathbf{K}_{\mathbf{p}} \mathbf{C}_{\mathbf{3}}^{-} \\
\left(\mathbf{V}_{\mathbf{R F}}\right)\end{array}$ & $\begin{array}{c}\mathbf{K}_{\mathbf{p}} \mathbf{T}_{\text {topo }} \\
\left(\mathbf{V}_{\mathbf{R F}}\right)\end{array}$ & $\begin{array}{c}\mathbf{K}_{\mathbf{p}} \mathbf{C}_{\mathbf{3}}^{-} \\
\left(\mathbf{V}_{\mathbf{G O P}}\right)\end{array}$ & $\begin{array}{c}\mathbf{K}_{\mathbf{p}} \mathbf{T}_{\text {topo }} \\
\left(\mathbf{V}_{\mathbf{G O P}}\right)\end{array}$ & $\mathbf{D e t}\left(\mathbf{K}_{\mathbf{p}}\right)$ \\
\hline 1 & 4000 & 2104 & 0.38 & 47.74 & $4.498 \mathrm{e}-4$ & -0.0153 & -0.003441 & 0.0510 & $-3.01 \mathrm{e}-5$ \\
\hline 2 & 3160 & 1731 & 0.88 & 49.34 & $1.6713 \mathrm{e}-3$ & -0.0261 & -0.012018 & 0.0550 & $-2.22 \mathrm{e}-4$ \\
\hline 3 & 2300 & 1331 & 4.81 & 47.82 & $1.6792 \mathrm{e}-2$ & -0.0226 & $-4.3175 \mathrm{e}-2$ & 0.0457 & $-2.08 \mathrm{e}-4$ \\
\hline 5 & 2240 & 1115 & 3.01 & 47.08 & $7.5501 \mathrm{e}-3$ & -0.0295 & $-2.0402 \mathrm{e}-2$ & 0.0751 & $-3.48 \mathrm{e}-5$ \\
\hline FD & 3532 & 1837 & 4.35 & 44.35 & $6.9795 \mathrm{e}-3$ & -0.0193 & $-1.8445 \mathrm{e}-2$ & 0.0461 & $-3.42 \mathrm{e}-5$ \\
\hline
\end{tabular}

Tabela 9.1 - Ganhos dos modelos identificados

\subsection{1 - Ajuste de sintonia dos controladores:}

Como mencionado acima, nesta primeira etapa foi feito o ajuste dos parâmetros de sintonia dos controladores, sendo o principal ajuste feito no fator de supressão das manipuladas. Para as demais etapas foi mantido o melhor ajuste alcançado nesta etapa.

Na tabela 9.2, apresentamos as principais características dos dois controladores:

\begin{tabular}{|l|l|l|l|}
\hline Controlador & Modelo nominal & Modelo planta & $\begin{array}{l}\text { Modelo de cálculo } \\
\text { da acão de controle }\end{array}$ \\
\hline QDMC & $\begin{array}{l}\text { Combinação dos modelos: 1 } \\
2345 \text { FD }\end{array}$ & FD (planta industrial) & Modelo nominal \\
\hline MMPC & $\begin{array}{l}\text { Combinação dos modelos: 1 } \\
2345 \text { FD }\end{array}$ & FD (planta industrial) & Modelos: 12345 \\
\hline
\end{tabular}

Tabela 9.2: Características dos controladores utilizados 
$\mathrm{Na}$ figura 9.1.a apresentamos os dois controladores com a mesma sintonia conforme tabela 9.3. Este caso corresponde a um ponto dentro da região mais freqüente de operação. Podemos observar nessa figura que o controlador MMPC é estável e o QDMC é instável, para um mesmo fator de supressão das manipuladas. Note na figura, que o QDMC vai aumentando a sua amplitude de oscilação à medida que o tempo passa, ao passo que no MMPC rapidamente chegamos a uma condição de estabilidade. Os números na abscissa de todos os gráficos representam o tempo decorrido em minutos.

\begin{tabular}{|c|c|c|}
\hline PARÂMETRO & QDMC & MMPC \\
\hline Período de amostragem & 1 min & 1 min \\
\hline $\begin{array}{c}\text { Instantes de cálculo da } \\
\text { predicão }\end{array}$ & $\begin{array}{l}1257101520253035404550 \\
6070\end{array}$ & $\begin{array}{l}12571015202530354045 \\
506070\end{array}$ \\
\hline Horizonte de controle & 2 & 1 para as duas CV's \\
\hline Peso das controladas & 1 para as duas CV's & 0.001 para as duas MV's \\
\hline Supressão das manipuladas & 0.001 para as duas MV's & \\
\hline
\end{tabular}

Tabela 9.3 - Parâmetros de sintonia dos controladores para figura 9.1.a

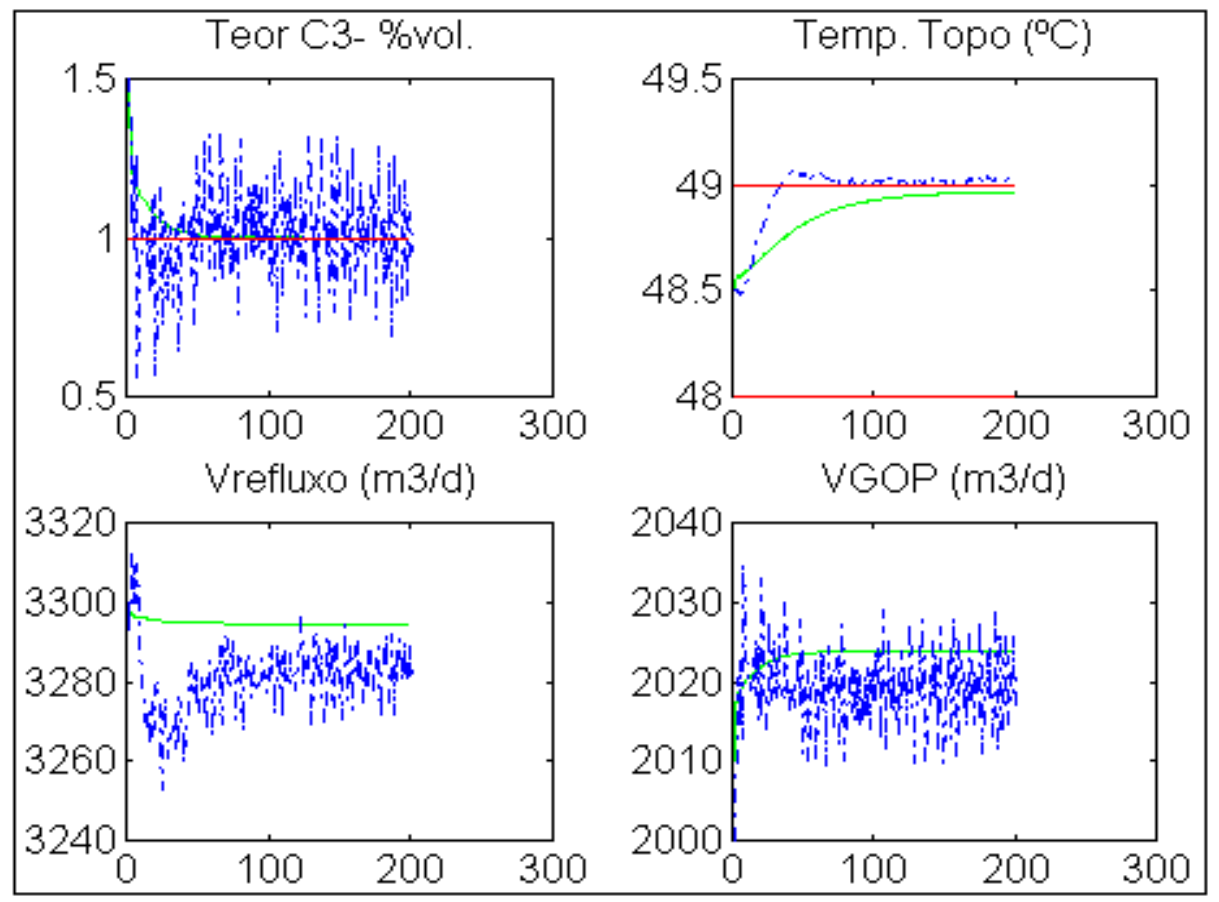

Figura 9.1.a - Comparação QDMC( - - ) x MMPC( — $)$ com mesma sintonia. 
Na figura 9.1.b apresentamos o QDMC com dois fatores de supressão diferentes, veja sintonia na tabela 9.4, onde pode se observar que para o controlador se estabilizar é necessário utilizar um fator de supressão 500 vezes maior que aquele utilizado para o MMPC, no entanto este fator apesar de ser maior que o utilizado para o MMPC não chega a ser um valor tão elevado.

\begin{tabular}{|c|c|c|}
\hline PARÂMETRO & QDMC & QDMC \\
\hline Período de amostragem & 1 min & 1 min \\
\hline $\begin{array}{c}\text { Instantes de cálculo da } \\
\text { predição }\end{array}$ & 12571015202530354045506070 & $\begin{array}{l}12571015202530354045 \\
506070\end{array}$ \\
\hline Horizonte controle & 2 & 1 para as duas CV's \\
\hline Peso das controladas & 1 para as duas CV's & 0.5 para as duas MV's \\
\hline $\begin{array}{c}\text { Supressão das } \\
\text { manipuladas }\end{array}$ & 0.001 para as duas MV's & \\
\hline
\end{tabular}

Tabela 9.4 - Parâmetros de sintonia dos controladores para figura 9.2.a

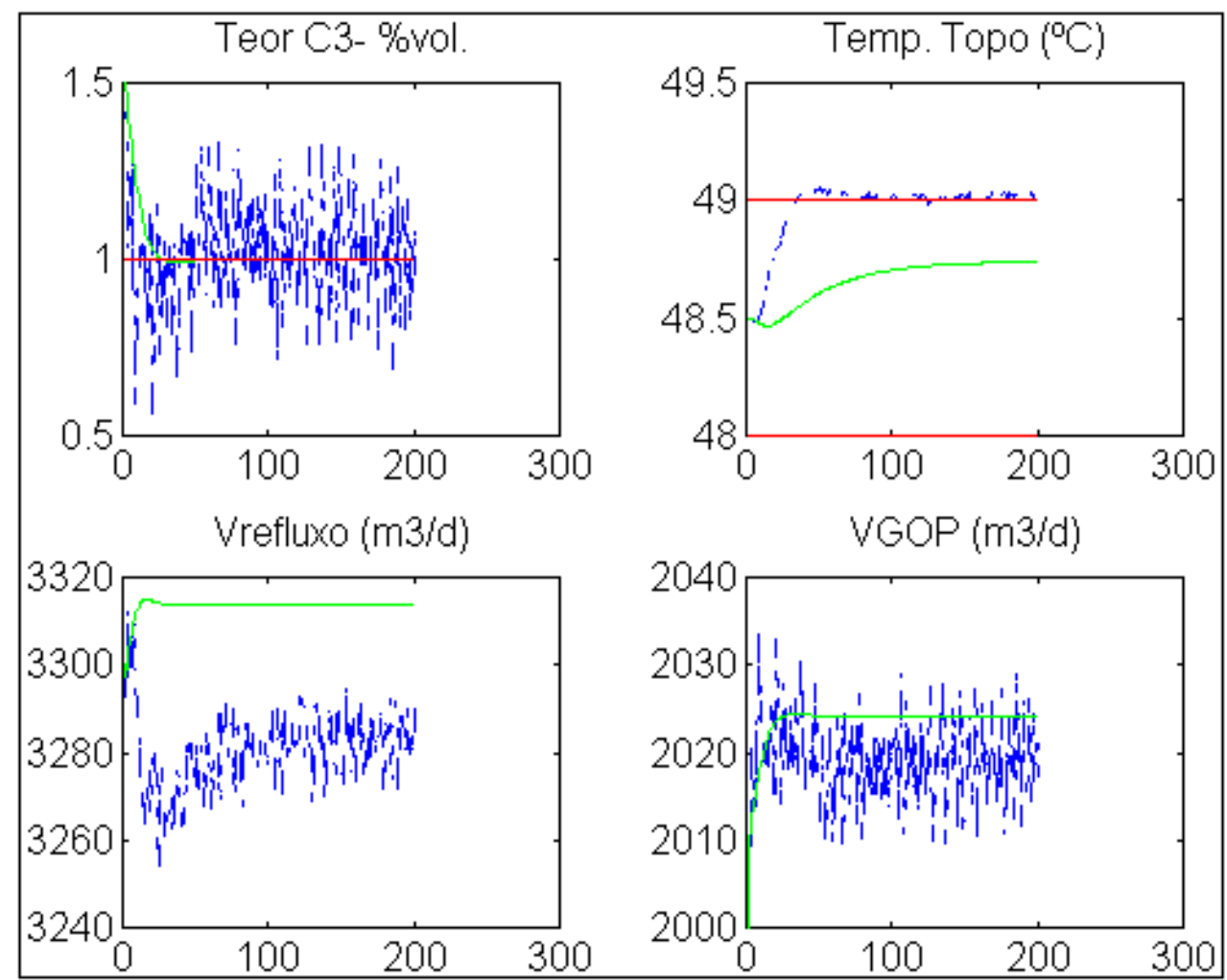

Figura 9.1.b - QDMCsupr $=0.001(--)$ QDMCsupr $=0.5(-)$.. 


\subsection{2-QDMC e MMPC com sintonias ajustadas :}

Nesta etapa foi avaliada a performance dos dois controladores frente a alterações nos set-points das variáveis controladas e também variações nas variáveis perturbadoras. Repete-se aqui a estrutura dos controladores apresentada na tabela 9.2. Na tabela 9.5 apresentamos a sintonia utilizada para os controladores.

\begin{tabular}{|c|c|c|}
\hline PARÂMETRO & MMPC & QDMC \\
\hline Período de Amostragem & 1 min & min \\
\hline $\begin{array}{c}\text { Instantes de cálculo da } \\
\text { predição }\end{array}$ & 12571015202530354045506070 & $\begin{array}{l}12571015202530354045 \\
506070\end{array}$ \\
\hline Horizonte de controle & 2 & 1 para as duas CV's \\
\hline Peso das controladas & 1 para as duas CV's & 0.5 para as duas MV's \\
\hline Supressão das \\
manipuladas
\end{tabular}

Tabela 9.5 - Sintonia dos controladores para item 9.1.2

Na figura 9.1.c apresentamos o comportamento do controlador quando alteramos o set-point do teor de $\mathrm{C}_{3}^{-}$. Podemos observar que os dois controladores têm praticamente a mesma performance, ou seja, atingem o novo "set-point" proposto com a mesma facilidade. 


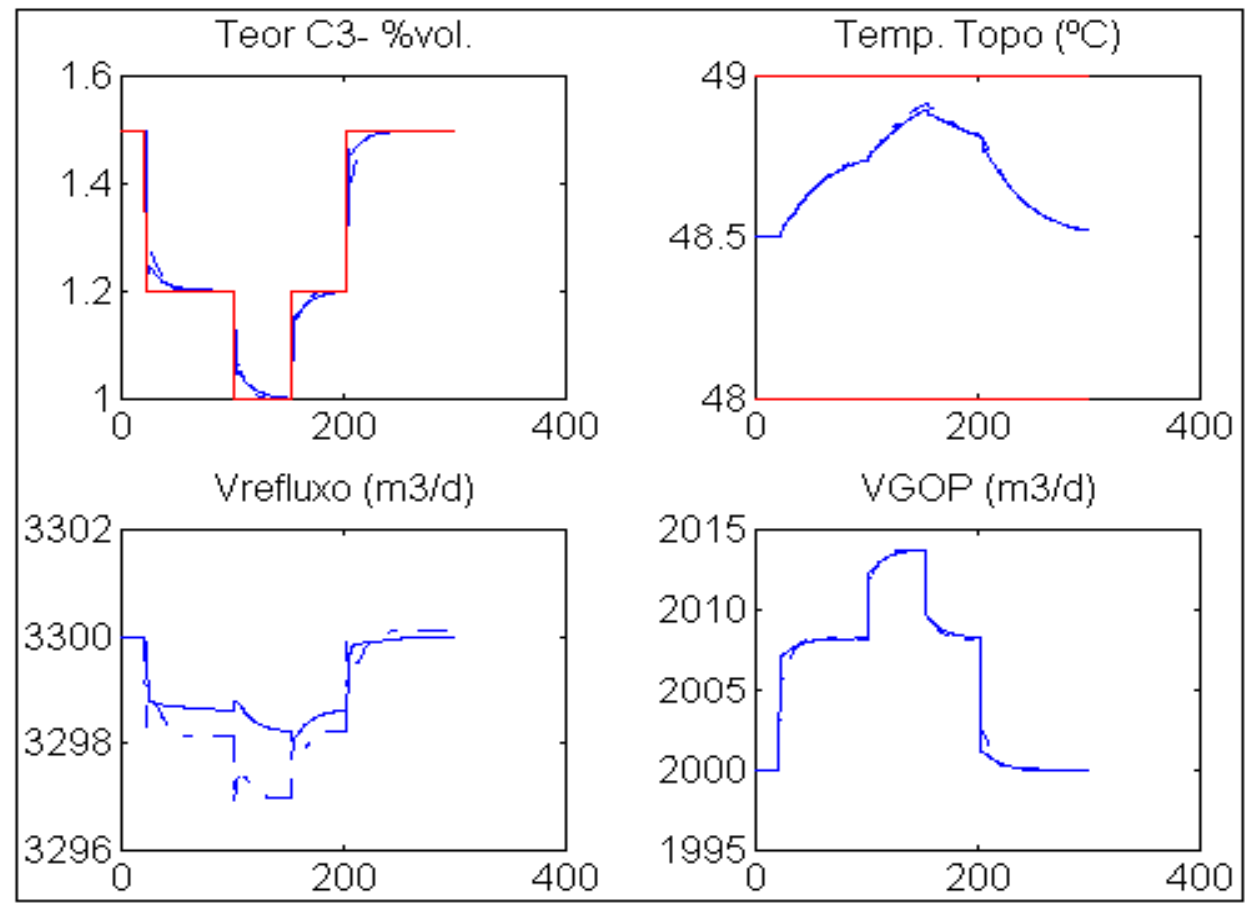

Figura 9.1.c - Alteração do "set-point" do teor de $\mathrm{C}_{3}{ }^{-} \mathrm{QDMC}($ - - - ) $\mathrm{MMPC}($ — ).

Na figura 9.1.d apresentamos o comportamento do controlador quando alteramos a faixa de controle da Ttopo em $-1{ }^{\circ} \mathrm{C}$ no instante 20 . Aqui no entanto, a performance do controlador MMPC é superior à do QDMC, o que pode ser constatado através da observação do tempo necessário para que a variável alcance a nova faixa de controle e o teor de $\mathrm{C}_{3}{ }^{-}$retorne para o "set-point". Com o MMPC o tempo de estabilização é em torno de 600 minutos ao passo que para o QDMC este tempo é superior a 1000 minutos. 


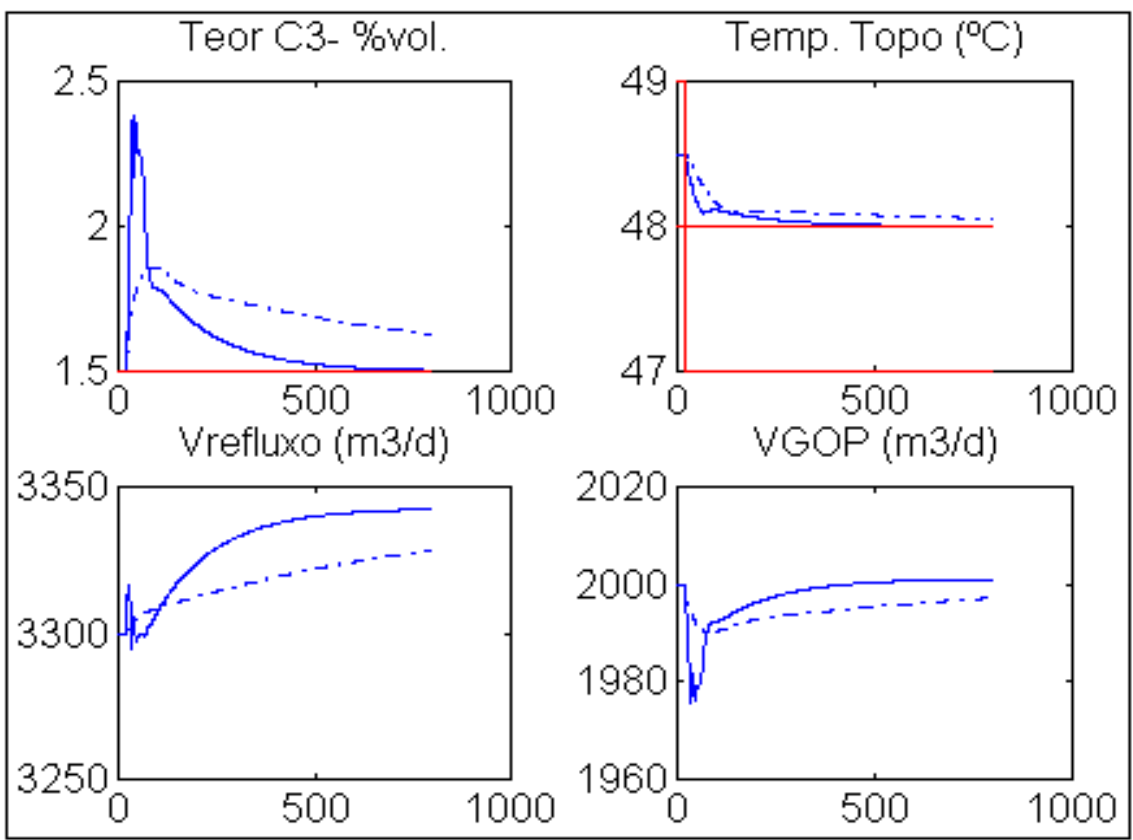

Figura 9.1.d - Alteração da faixa de controle da Ttopo QDMC( - - ) MMPC( — ).

Na figura 9.1.e apresentamos a performance dos controladores quando aplicamos variações em degrau na vazão de carga para a coluna, que é uma variável perturbadora do processo. O degrau aplicado foi de $50 \mathrm{~m}^{3} / \mathrm{d}$ no instante $30 \mathrm{~min}$. Neste caso, podemos observar que os dois controladores conseguem manter as variáveis controladas no "setpoint" e faixa de controle respectivamente, sem grandes dificuldades.

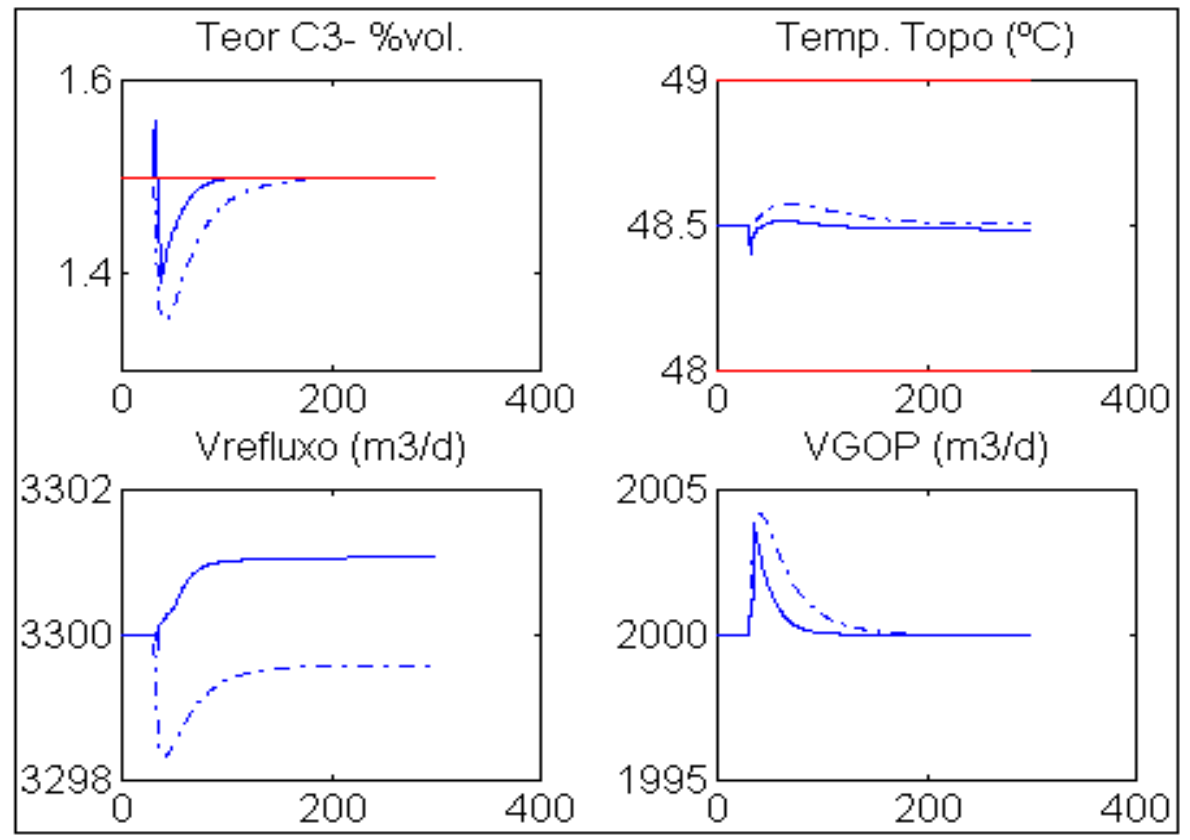

Figura 9.1.e - Aplicação de degrau na vazão de carga QDMC( - - - ) MMPC( — ). 
Na figura 9.1.f apresentamos a performance dos controladores quando aplicamos variações em degrau na temperatura do fluido de aquecimento para o refervedor, que é uma variável perturbadora do processo. $\mathrm{O}$ degrau aplicado foi de $5^{\circ} \mathrm{C}$ no instante 30 min. Neste caso, podemos observar que os dois controladores conseguem manter as variáveis controladas no "set-point" e faixa de controle respectivamente, sem grandes dificuldades.

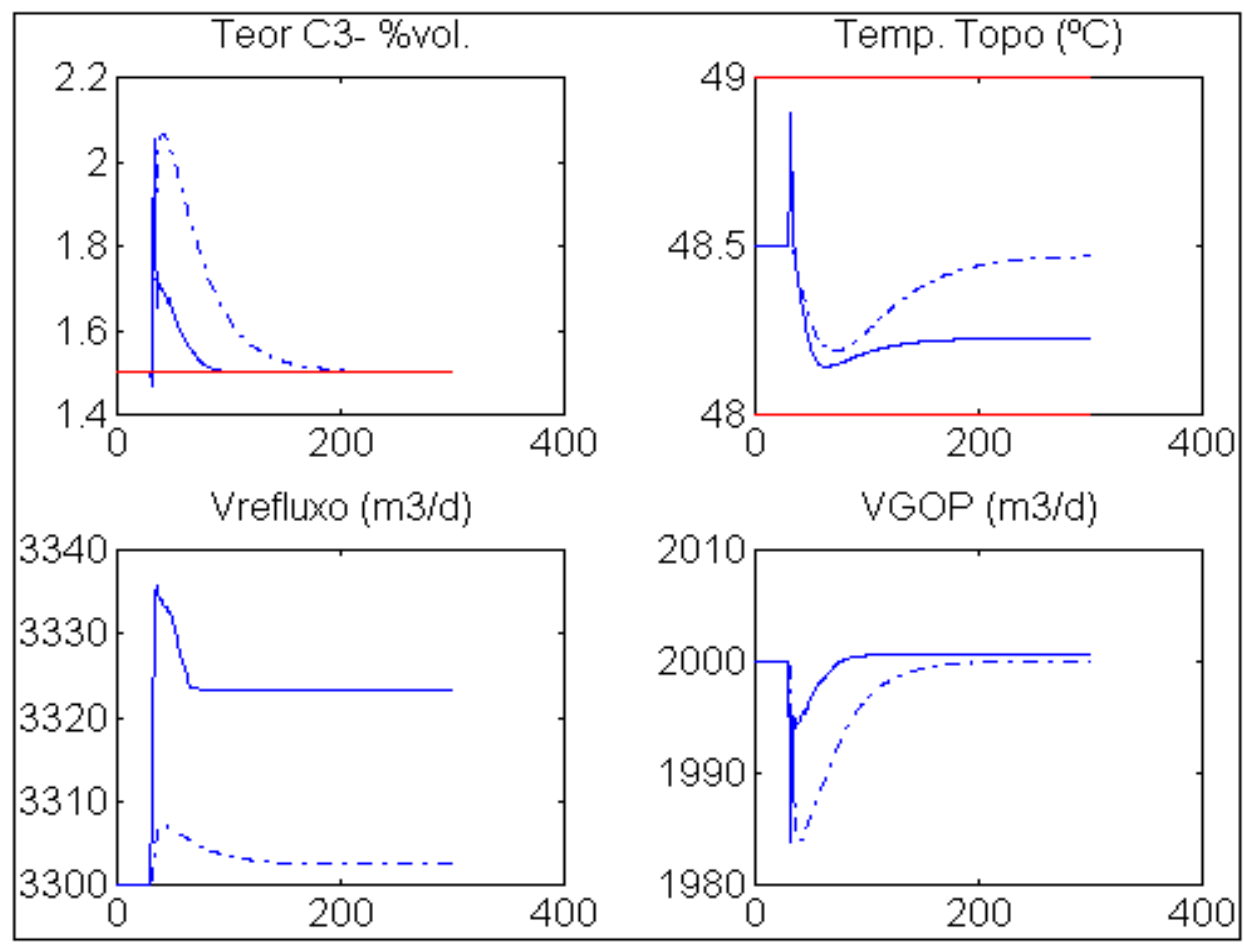

Figura 9.1.f - Aplicação de degrau na TGOP QMDC( - - - ) MMPC( — ).

\subsection{3 - Alteração do modelo da planta:}

Nesta etapa analisamos a performance dos controladores frente a alterações no modelo utilizado para a planta. As avaliações consistiram em colocar o controlador em operação tendo como modelo da planta aquele da planta industrial e no instante 20 min. o modelo da planta era alterado para um novo modelo. Os modelos utilizados foram todos aqueles levantados através do simulador, ou seja, 1, 2, 3, 4 e 5. Em todos os casos analisados, sempre partimos com o teor de $\mathrm{C}_{3}{ }^{-}$acima do "set-point". A sintonia do 
controlador permaneceu a mesma do item anterior (veja tabela 9.5). Os casos analisados são apresentados nas figuras 9.1.g, 9.1.h, 9.1.i, 9.1.j e 9.1.k. A seguir analisamos a performance para a alteração de cada modelo.

$\mathrm{Na}$ figura 9.1.g apresentamos o comportamento dos controladores quando mudamos o modelo da planta para o modelo 1. Podemos observar que o MMPC consegue levar o teor $\mathrm{C}_{3}^{-}$para o "set-point" apesar de levar um tempo maior do que quando não temos alteração no modelo da planta (veja figura 9.1.c). Já o QDMC não consegue levar o teor de $\mathrm{C}_{3}{ }^{-}$para o "set-point" apresentando assim um "off-set". $\mathrm{O}$ mesmo ocorre com a Ttopo.

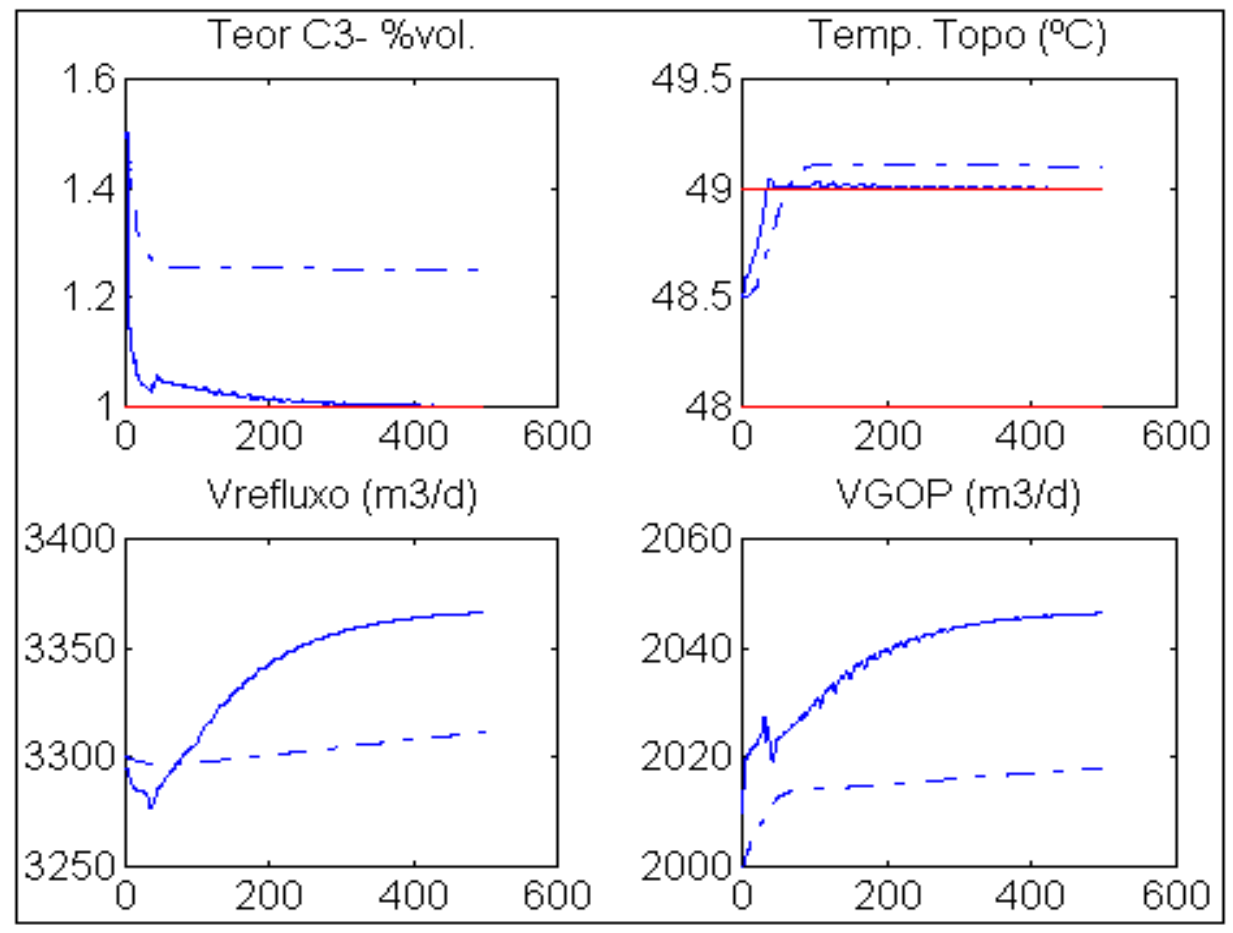

Figura 9.1.g - Mudança p/ modelo 1 no instante 20 min., QDMC( - - - ) MMPC( — ).

$\mathrm{Na}$ figura 9.1.h apresentamos o comportamento dos controladores quando mudamos o modelo da planta para o modelo 2. Novamente aqui temos um comportamento semelhante ao apresentado quando alteramos o modelo da planta para o modelo 1. No entanto, o tempo de estabilização para o MMPC é superior àquele quando não temos alteração do modelo da planta (figura 9.1c). O QDMC neste caso também apresenta "off-set" para as variáveis controladas. 


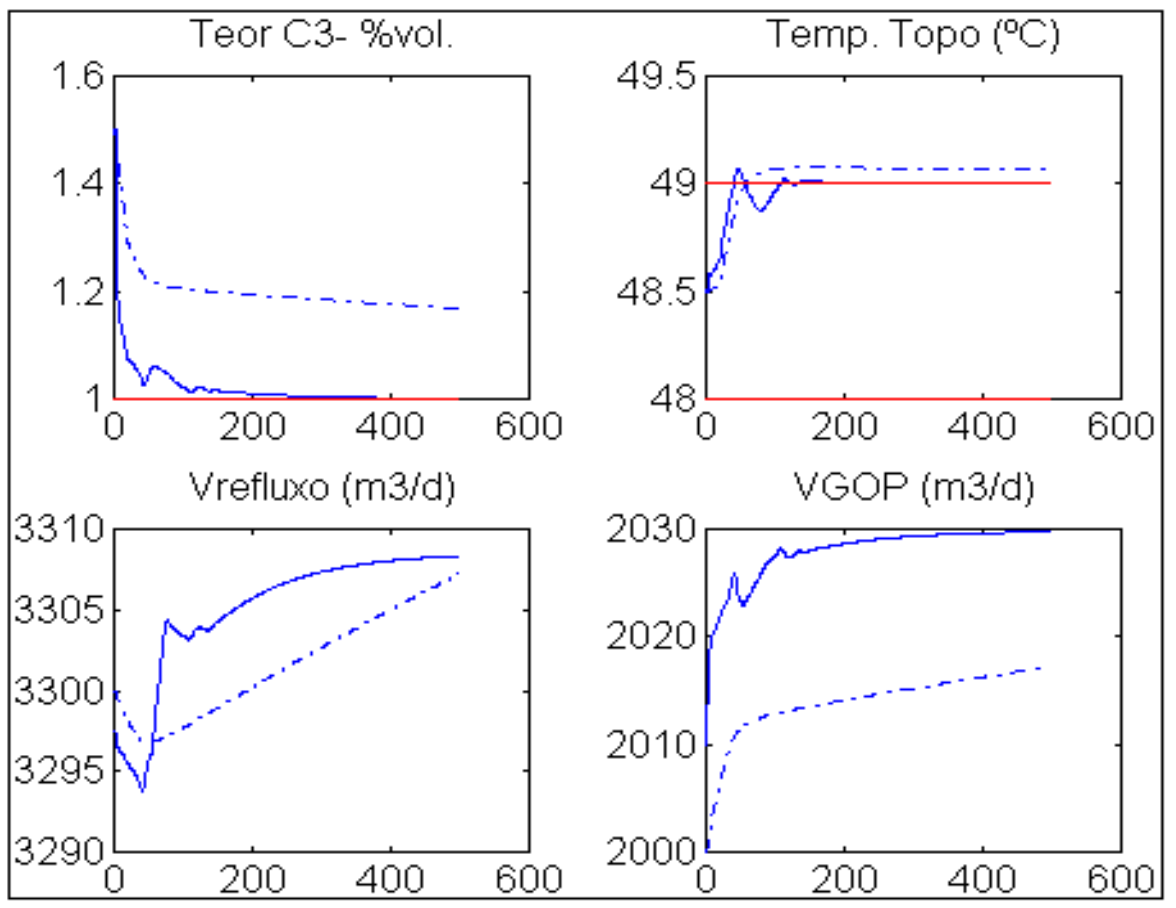

Figura 9.1.h - Mudança p/ modelo 2 no instante 20 min., QMDC( - - - ) MMPC( — ).

$\mathrm{Na}$ figura 9.1.i apresentamos o comportamento dos controladores quando mudamos o modelo da planta para o modelo 3. Neste caso o comportamento do MMPC é semelhante àquele apresentando na figura 9.1.c. No entanto, o QDMC apresenta um ligeiro "off-set" para o teor de $\mathrm{C}_{3}{ }^{-}$o que não chega a comprometer sua performance.

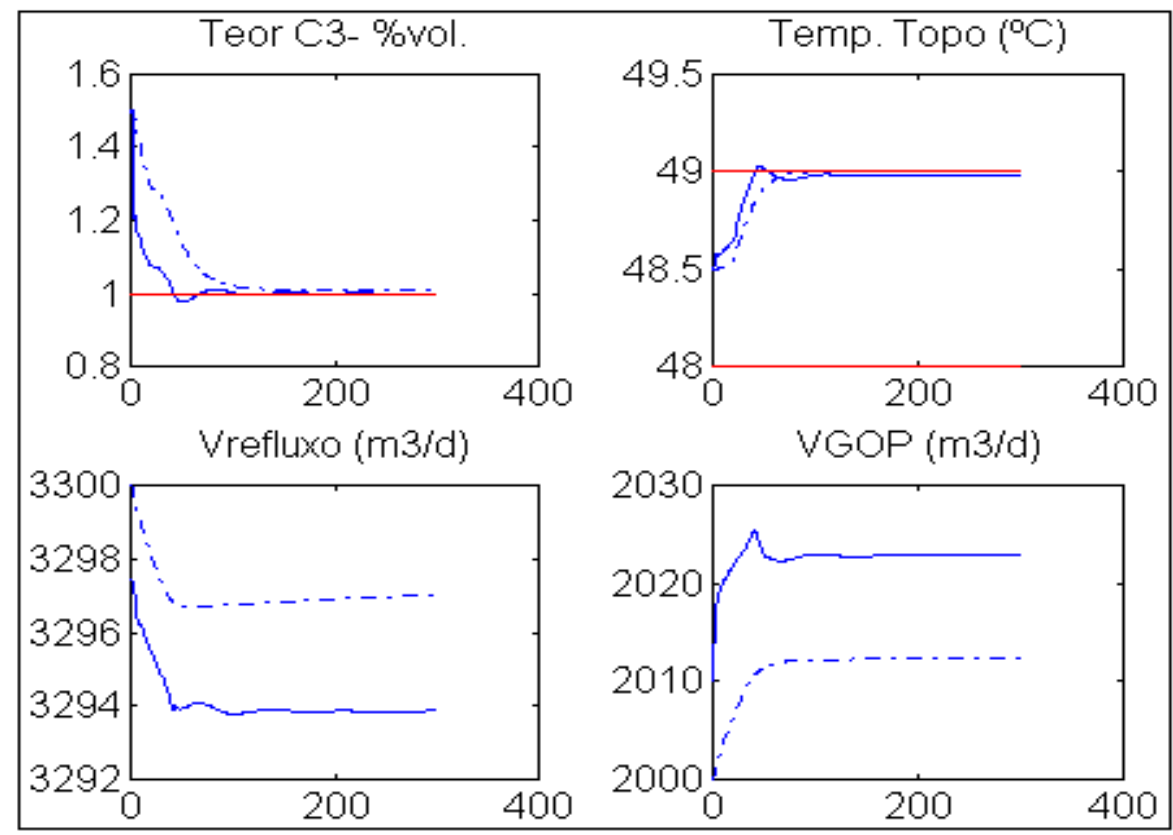

Figura 9.1.i - Mudança para modelo 3 no instante 20 min., QMDC( - - ) MMPC( - ). 
$\mathrm{Na}$ figura 9.1.j apresentamos o comportamento dos controladores quando mudamos o modelo da planta para o modelo 4. Aqui no entanto, os dois controladores apresentaram "off-set" para o teor $\mathrm{C}_{3}{ }^{-}$, sendo que para o MMPC o "off-set" é menor do que aquele apresentado pelo QDMC. Para a Ttopo somente o QDMC apresentou "offset” sendo que o MMPC levou a Ttopo para seu limite máximo.

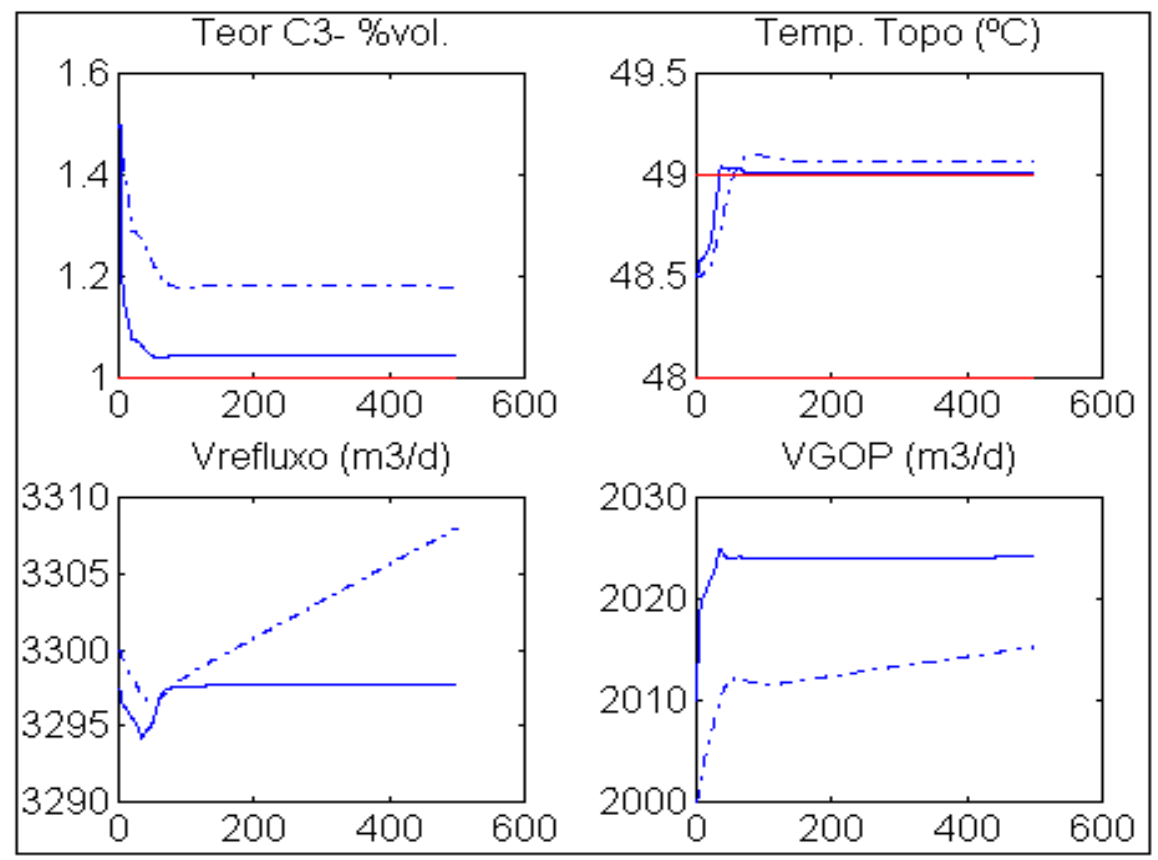

Figura 9.1.j - Mudança para modelo 4 no instante 20min., QDMC( - - ) $\mathrm{MMPC}($ - ).

$\mathrm{Na}$ figura 9.1.k apresentamos o comportamento dos controladores quando mudamos o modelo da planta para o modelo 5. Temos neste caso um comportamento semelhante ao apresentado quando alteramos o modelo da planta para o modelo 2, ou seja, o MMPC consegue levar o teor de $\mathrm{C}_{3}$ " para o "set-point" e a Ttopo para seu limite superior, o que não ocorre com o QDMC, que apresenta "off-set" para as duas variáveis controladas. 


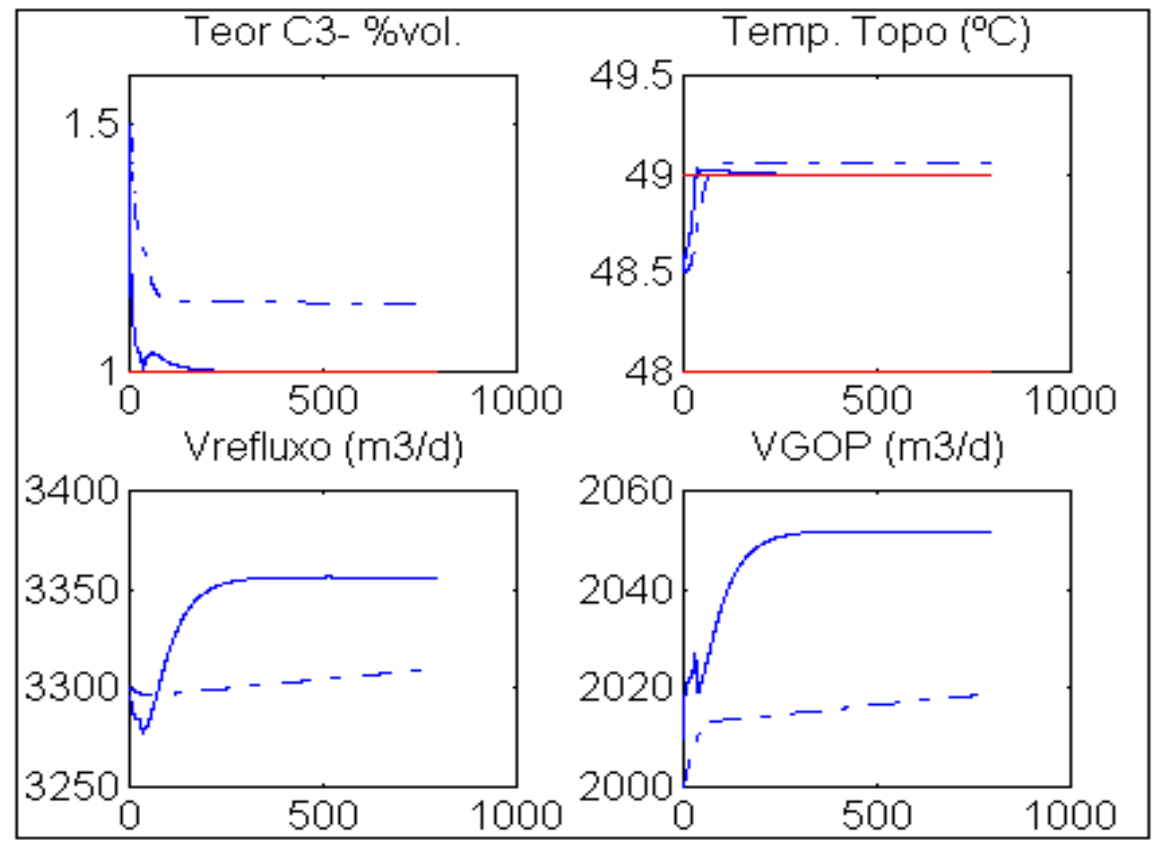

Figura 9.1.k - Mudança modelo p/ 5 no instante 20 min., QDMC( - - ) MMPC( — ).

De forma geral, podemos constatar que o QDMC necessita de fatores de supressão superiores ao MMPC para estabilizar o sistema de controle. Após sintonizado, o QDMC apresentou, em alguns casos, comportamento semelhante ao MMPC (figuras 9.1.c, 9.1.e, 9.1.f) quando o modelo da planta não se alterava. No entanto, quando tivemos alteração no modelo da planta, o que no processo real equivale a termos alteração do ponto de operação, o QDMC se mostrou ineficaz em controlar o processo, apresentando "off-set" na maioria das situações simuladas (figuras 9.1.g, 9.1.h, 9.1.i, 9.1.k). Assim, podemos concluir que o QDMC não seria adequado para controle do nosso processo, ao passo que o MMPC se mostrou mais adequado, apresentando melhor performance que o QDMC.

Tendo em vista que o controlador MMPC se mostrou mais eficiente que o QDMC, o mesmo foi implementado para controle da planta industrial. No próximo item apresentamos a performance do MMPC em operação na unidade industrial.

\section{2 - Implantação do controlador MMPC na planta industrial}

O controlador MMPC entrou em operação na planta industrial no dia 14 de fevereiro de 2001, e tem operado continuamente demonstrando uma grande aceitação 
por parte dos operadores da unidade. Para implantação do controlador na planta industrial foi utilizado o SICON ( Sistema Integrado de Controle da Petrobras ). Este sistema é descrito no item 9.2.1. As subrotinas do SICON tiveram que ser adaptadas para permitir a montagem do controlador na forma paramétrica apresentada no item 8.2.1, principalmente na parte de leitura dos modelos. Também foram implementadas novas subrotinas no sistema para permitir o cálculo das ações de controle através do SQP.

Para verificação da performance do controlador em operação na planta industrial, foram coletados dados com o controlador em operação sujeito às variações normais da planta tais como: variáveis perturbadoras medidas (vazão de carga e temperatura do fluído de aquecimento) e variáveis perturbadoras não medidas (composição da carga, variações ambientais etc.). Também foram realizados testes para verificação da operação do controlador frente à alterações no "set-point" das variáveis controladas.

As figuras apresentadas abaixo foram obtidas através da interface gráfica do $\mathrm{PI}^{\mathrm{TM}}$ (Plant Information) da Oil System Inc, que é o sistema de gerenciamento de dados de processo existente na RPBC. Este sistema consiste de uma interface de leitura dos dados do SDCD enviando-os para um servidor onde fica a base de dados. Esta base de dados pode ser acessada para gerar gráficos através da interface gráfica do próprio programa e também através da planilha eletrônica $\operatorname{Excel}^{\mathrm{TM}}$ da Microsoft, permitindo uma análise detalhada de todas as variáveis operacionais do processo.

Para um melhor entendimento das figuras apresentaremos a seguir uma descrição de cada item constante das mesmas:

$\checkmark$ Geral para todas as figuras:

- campo superior esquerdo: é uma descrição da variável

- o número abaixo dos “tag's" é o valor da variável de processo (PV) no caso das variáveis controladas e perturbadoras, e o "set-point" (SV) no caso das manipuladas. Este é o valor real lido do SDCD e se refere ao instante de tempo apresentado no lado direito inferior da figura.

- parte inferior: são apresentados os instantes inicial e final do período de tempo em que a variável é observada. 
- a escala que aparece do lado esquerdo dos gráficos representa a faixa de operação atual das variáveis.

$\checkmark$ Variável controlada teor de $\mathrm{C}_{3}^{-}$:

- AI2430 é o “tag” da variável de processo (PV).

- AI2430MN é o "tag” do limite mínimo da variável para controle.

- AI2430MX é o "tag” do limite máximo da variável para controle.

Variável controlada Ttopo:

- TC2483 é o "tag” da variável de processo (PV).

- TC2483MN é o "tag” do limite mínimo da variável para controle.

- TC2483MX é o "tag” do limite máximo da variável para controle.

$\checkmark$ Variável manipulada Vrefluxo:

- FC2454SV é o "tag" do "set-point" (SV) da variável.

- FC2454SMN é o "tag" do limite mínimo do "set-point" (SV) para controle.

- FC2454SMX é o "tag" do limite máximo do "set-point" (SV) para controle.

- FC2454MD é o modo da variável manipulada no SDCD. Informa se a variável está liberada para ser utilizada pelo controle supervisório. A opção RCAS (cascata remota) indica que o "set-point" está sendo fornecido pelo controle supervisório.

$\checkmark$ Variável manipulada VGOP:

- FC24151SV é o "tag” do "set-point” (SV) da variável.

- FC24151SMN é o "tag" do limite mínimo do "set-point" (SV) para controle.

- FC24151SMX é o "tag" do limite máximo do "set-point" (SV) para controle.

- FC24151MD é o modo da variável manipulada no SDCD. Informa se a variável está liberada para ser utilizada pelo controle supervisório. A 
opção RCAS (cascata remota) indica que o "set-point" está sendo fornecido pelo controle supervisório.

$\checkmark$ Variáveis perturbadoras (Vcarga e TGOP):

- FC2452SV ou TI24154 são os "tags" das variáveis perturbadoras medidas, que são utilizadas no controle.

Na tabela 9.6 apresentamos os parâmetros de sintonia utilizados no controlador utilizado na planta industrial.

\begin{tabular}{|c|c|}
\hline PARÂMETRO & VALOR \\
\hline Período de amostragem & $1 \mathrm{~min}$ \\
\hline Instantes de cálculo da predição & 12571015202530354045506070 \\
\hline Horizonte de controle & 2 \\
\hline Peso das controladas & 1 para as duas CV's \\
\hline Supressão de movimentos & 0.01 para as duas MV's \\
\hline Modelo nominal & Combinação dos modelos: 12345 FD \\
\hline $\begin{array}{l}\text { Modelos para cálculo da ação de } \\
\text { controle }\end{array}$ & 12345 \\
\hline
\end{tabular}

Tabela 9.6 - Parâmetros de sintonia do controlador industrial

A seguir analisaremos os gráficos referentes aos casos estudados com o controlador em operação na planta industrial.

O primeiro conjunto de gráficos (figuras 9.2.a1 a 9.2.a3) representa um período de operação do controlador onde não temos alteração do "set-point" das variáveis controladas. $\mathrm{O}$ teor de $\mathrm{C}_{3}$ " é controlado no "set-point", $(\mathrm{AI} 2430 \mathrm{MN}=\mathrm{AI} 2430 \mathrm{MX}=0.9$ $\%$ vol.), enquanto que a Ttopo é controlada dentro de uma faixa $\left(\mathrm{TC} 2483 \mathrm{MN}=49^{\circ} \mathrm{C}\right.$ e TC2483MX $=50^{\circ} \mathrm{C}$ ), as variáveis manipuladas têm seus limites bastante amplos (vazão de refluxo de 2000 a $4300 \mathrm{~m}^{3} / \mathrm{d}$, vazão de GOP de 500 a $2300 \mathrm{~m}^{3} / \mathrm{d}$ ). Estes limites das variáveis manipuladas fazem com que o controlador tenha ampla liberdade para atuar no controle do processo. Podemos observar nessas figuras que o controlador consegue manter as variáveis controladas nas suas especificações ("set-point" para o $\mathrm{C}_{3}$ " e faixa de controle para a Ttopo), mesmo quando temos variações significativas nos valores das 
variáveis perturbadoras. Veja que em alguns momentos a vazão de carga (Fig. 9.2.a3) chega a variar $250 \mathrm{~m}^{3} / \mathrm{d}$ em um curto intervalo de tempo.

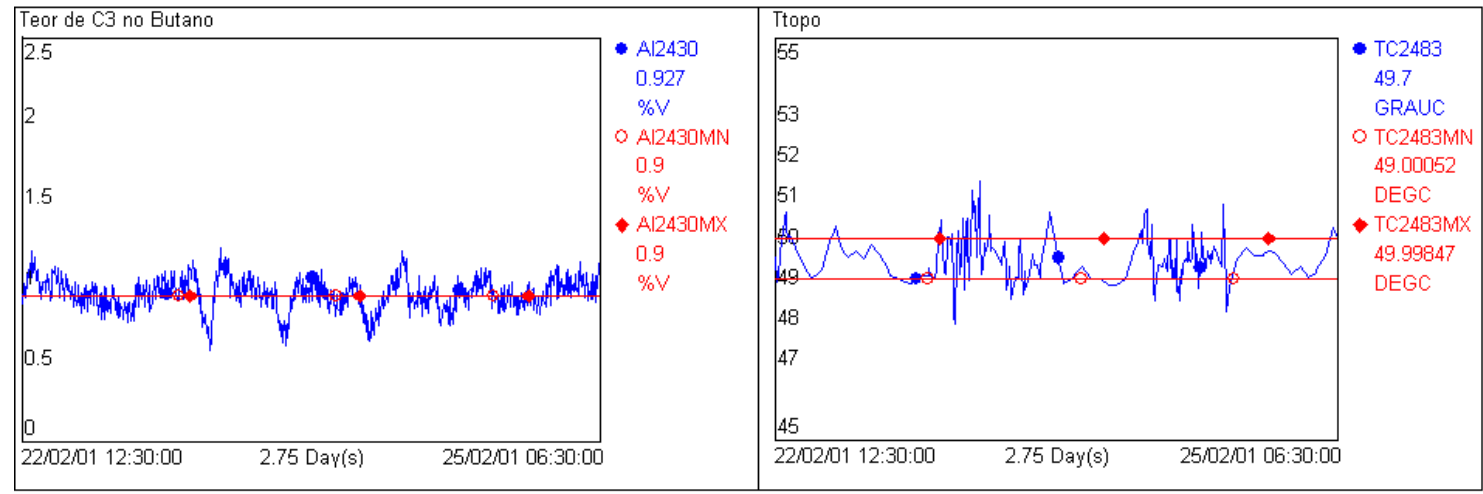

Figura 9.2.a1 - Variáveis controladas em operação típica.

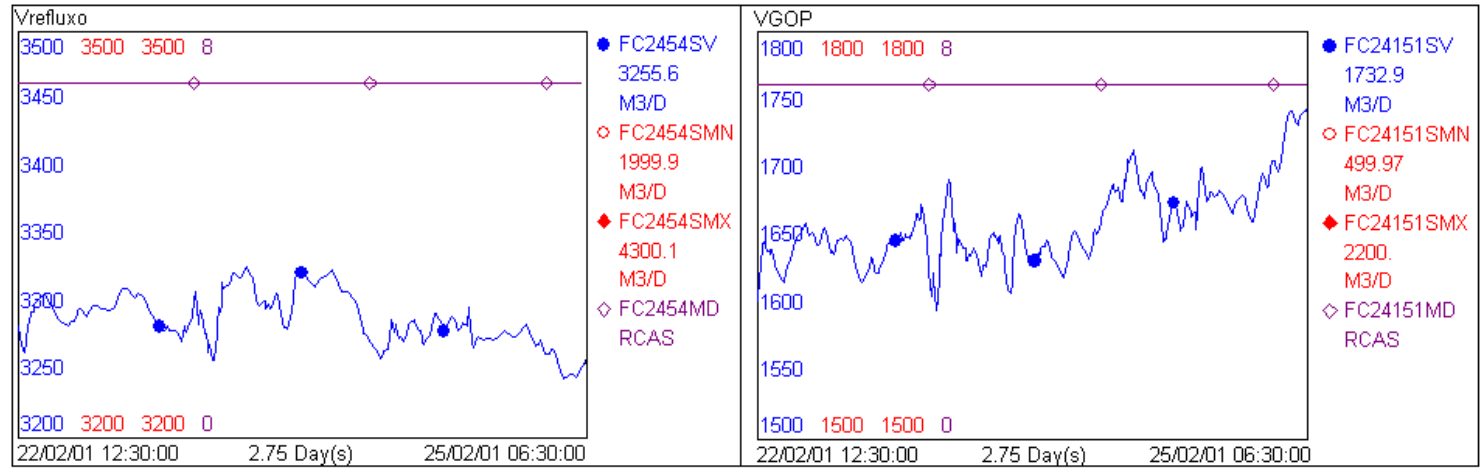

Figura 9.2.a2 - Variáveis manipuladas em operação típica.

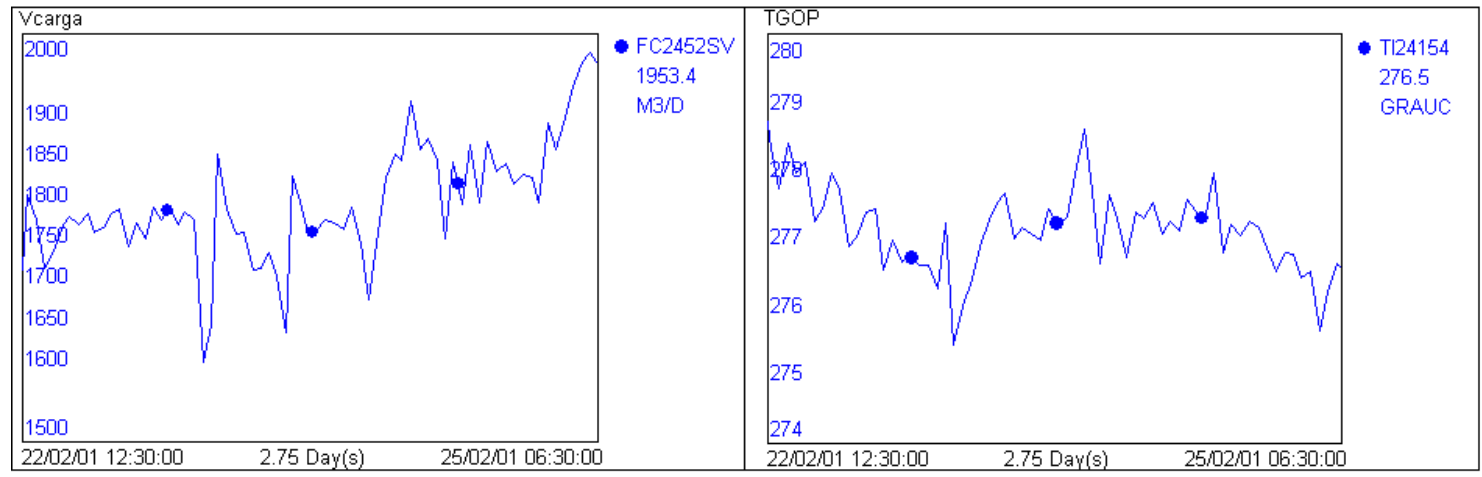

Figura 9.2.a3 - Variáveis perturbadoras em operação típica.

No próximo conjunto de gráficos (figuras 9.2.b1 a 9.2.b3) apresentamos dados relativos a um teste (teste 1), realizado para observar a performance do controlador frente a alterações no "set-point" do teor de $\mathrm{C}_{3}{ }^{-}$no butano. $\mathrm{O}$ degrau aplicado foi de 0.3 \%vol., passando de 1.2 para $1.5 \%$ vol.. Podemos observar que o novo patamar de "setpoint" é atingido em aproximadamente 30 minutos. Observe que não ocorre violação da 
faixa de controle da temperatura de topo mesmo com severas variações nas variáveis perturbadoras como a vazão de carga que variou quase $200 \mathrm{~m}^{3} / \mathrm{d}$ durante o período de teste.

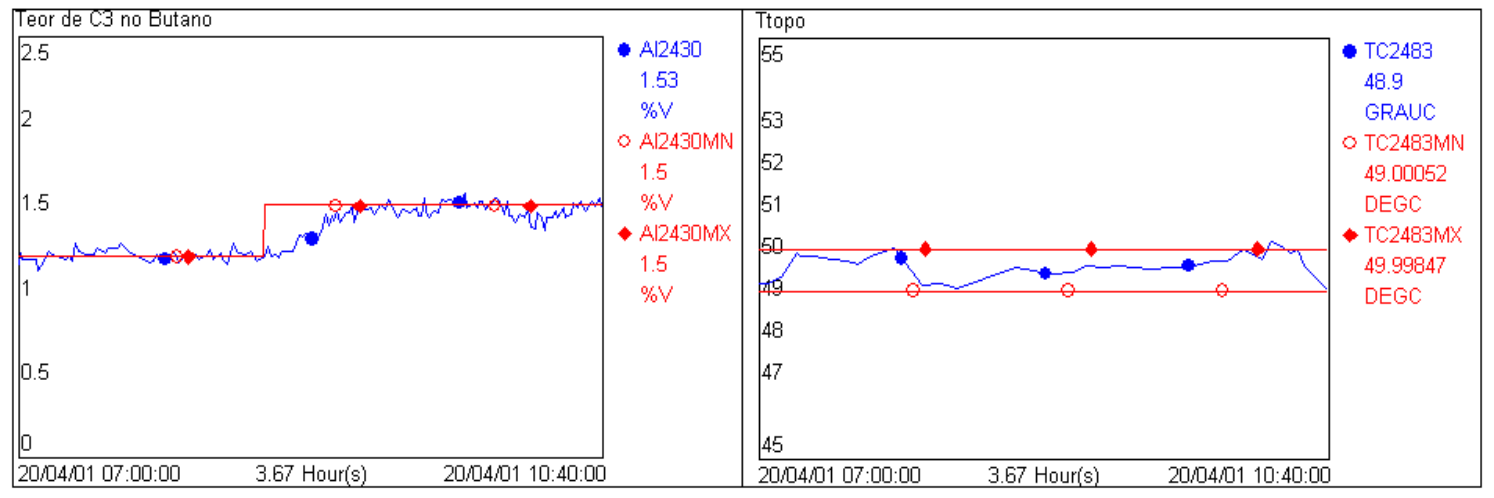

Figura 9.2.b1 - Variáveis controladas teste 1.

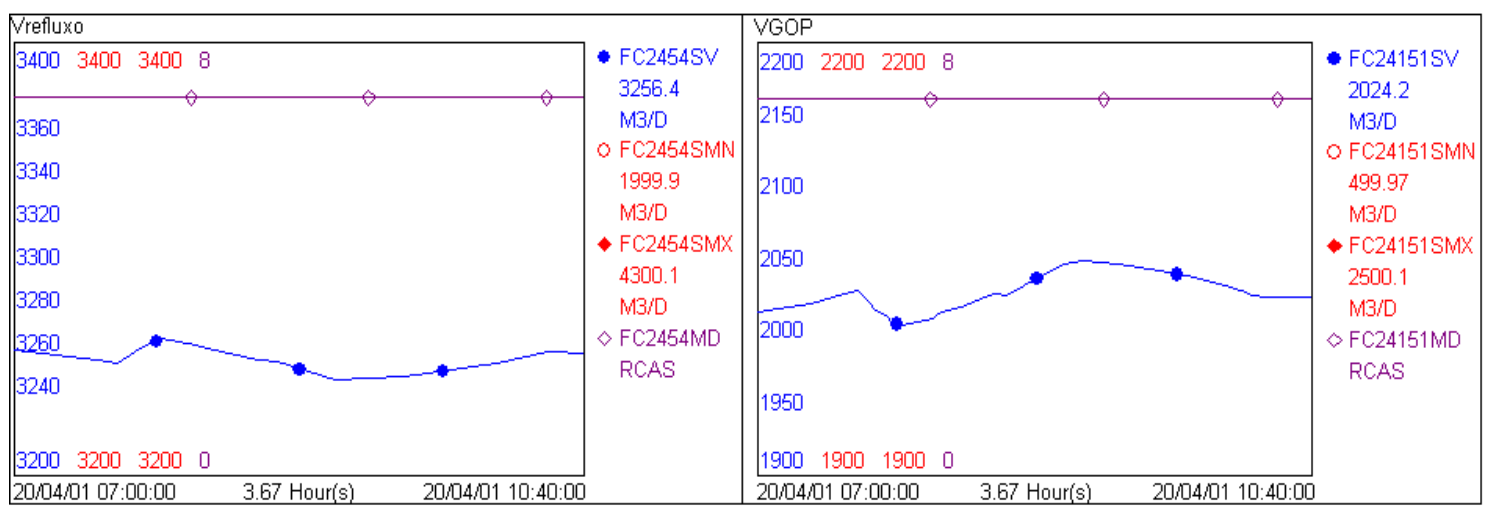

Figura 9.2.b2 - Variáveis manipuladas teste 1.

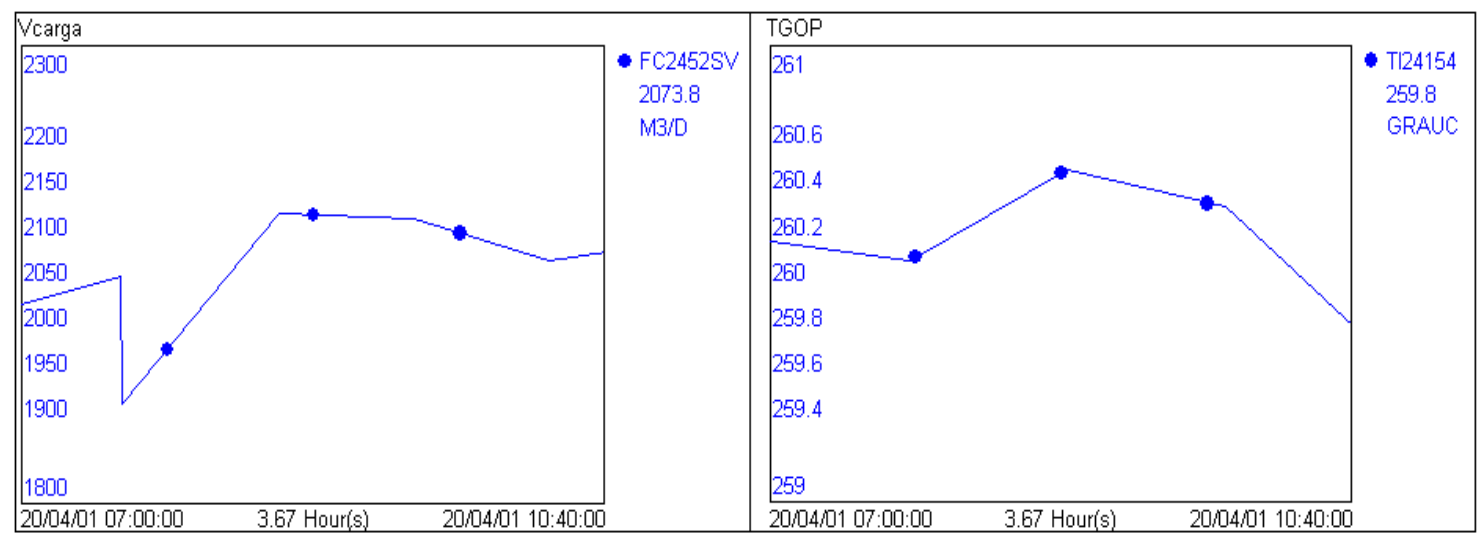

Figura 9.2.b3 - Variáveis perturbadoras teste 1.

O segundo teste realizado na planta (teste 2), é descrito pelas figuras 9.2.c1 a 9.2.c3. Ele consistiu novamente na alteração do "set-point" do teor de $\mathrm{C}_{3}{ }^{-}$no butano. $\mathrm{O}$ degrau aplicado foi de $0.3 \%$ vol., só que desta vez no sentido contrário ao anterior, 
passando o "set-point" $1.2 \%$ vol. para $0.9 \%$ vol. Observe que quase simultaneamente à aplicação do degrau no "set-point" tivemos uma grande variação na vazão de carga da coluna sendo que a mesma saiu de um patamar de $2050 \mathrm{~m}^{3} / \mathrm{d}$ baixou para $1800 \mathrm{~m}^{3} / \mathrm{d}$, e depois foi elevada para $2500 \mathrm{~m}^{3} / \mathrm{d}$ e finalmente retornou para o patamar de $2000 \mathrm{~m}^{3} / \mathrm{d}$. Estas variações ocorreram durante um período de aproximadamente 4 horas. Observe que tivemos também uma alteração no patamar da temperatura do fluido de aquecimento passando a mesma de $259^{\circ} \mathrm{C}$ para em torno de $261^{\circ} \mathrm{C}$. Veja que durante a ocorrência dessas perturbações o teor de $\mathrm{C}_{3}{ }^{-}$oscilou entre um máximo a $1.6 \%$ vol. e um mínimo de $0.7 \%$ vol.. A Ttopo violou $1^{\circ} \mathrm{C}$ acima do limite máximo de controle. Observe que as variáveis manipuladas variaram bastante para manter as variáveis controladas dentro de suas especificações durante a ocorrência das perturbações, permanecendo o tempo todo ligadas (RCAS).

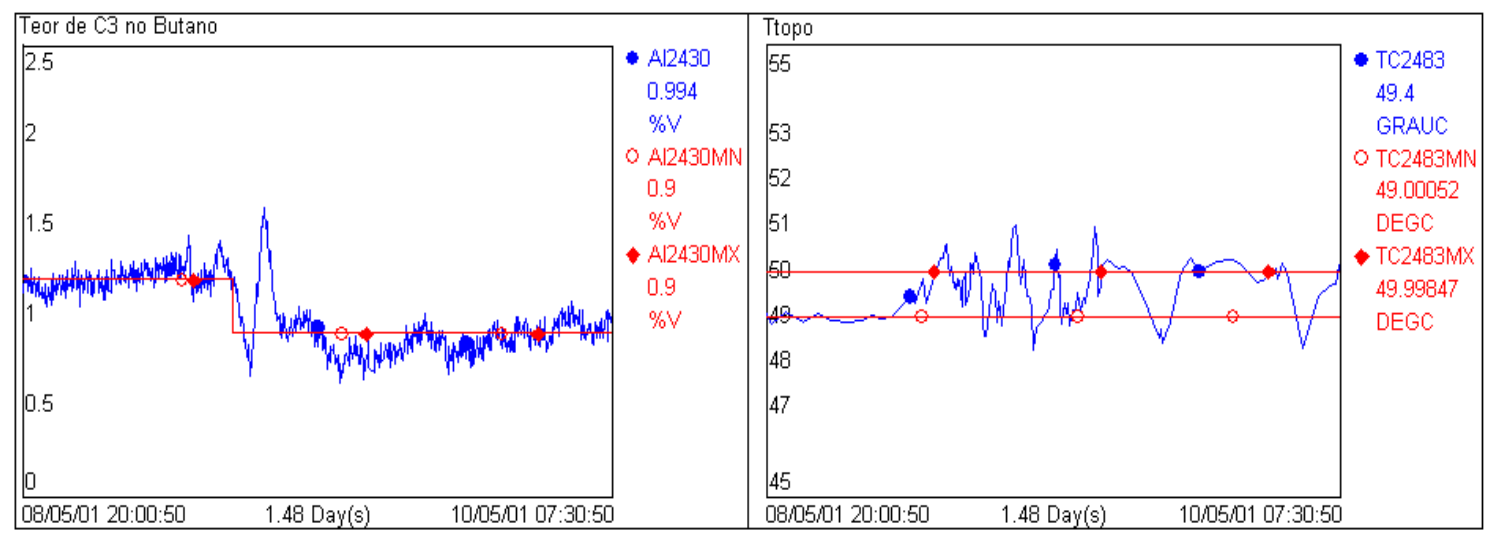

Figura 9.2.c1 - Variáveis controladas teste 2.

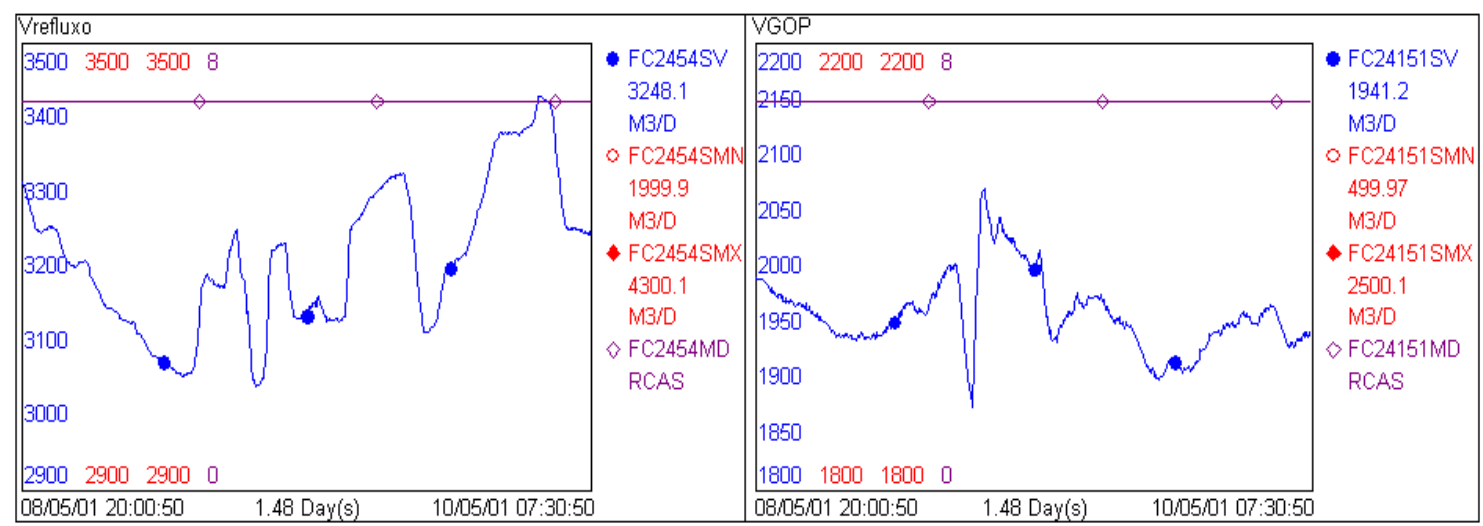

Figura 9.2.c2 - Variáveis manipuladas teste 2. 


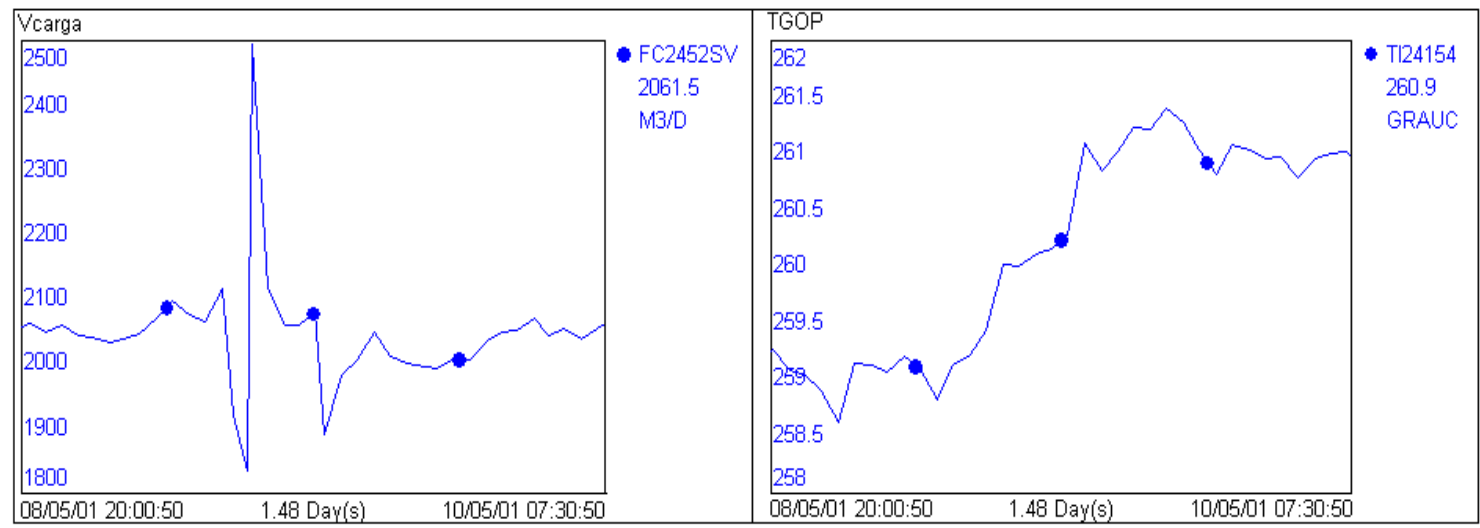

Figura 9.2.c3 - Variáveis perturbadoras teste 2.

O terceiro teste (teste3), corresponde às figuras 9.2.d1 a 9.2.d3. Ele consistiu na aplicação de um degrau nos limites da temperatura de topo (Ttopo), permanecendo o "set-point" do $\mathrm{C}_{3}$ " no mesmo valor. O degrau aplicado foi de $0.5^{\circ} \mathrm{C}$ nos limites da Ttopo, passando o máximo de $50^{\circ} \mathrm{C}$ para $49.5^{\circ} \mathrm{C}$ e o mínimo de $49^{\circ} \mathrm{C}$ para $48.5^{\circ} \mathrm{C}$. Observe que o controlador em poucos minutos leva a Ttopo para dentro na nova faixa permanecendo $\mathrm{o} \mathrm{C}_{3}$ - no "set-point" especificado. Veja que o patamar da vazão de refluxo muda da faixa de $2800 \mathrm{~m}^{3} / \mathrm{d}$ para um patamar de $2950 \mathrm{~m}^{3} / \mathrm{d}$ sendo que a vazão de fluido de aquecimento sofre um ligeiro acréscimo. Após a estabilização inicial da temperatura na nova faixa de controle, observamos que as variáveis manipuladas mudam de patamar, passando a vazão de refluxo de $2950 \mathrm{~m}^{3} / \mathrm{d}$ para $3100 \mathrm{~m}^{3} / \mathrm{d}$ e a vazão de fluido de aquecimento de $1900 \mathrm{~m}^{3} / \mathrm{d}$ para $2050 \mathrm{~m}^{3} / \mathrm{d}$. Esta mudança de patamar provavelmente ocorreu devido à alteração principalmente nas variáveis perturbadoras não medidas sendo a mais importante delas a qualidade da carga para a coluna. As variáveis perturbadoras medidas não tiveram alterações significativas no período. Esta mudança no patamar das variáveis manipuladas foi necessário para que as variáveis controladas mantivessem suas especificações. 


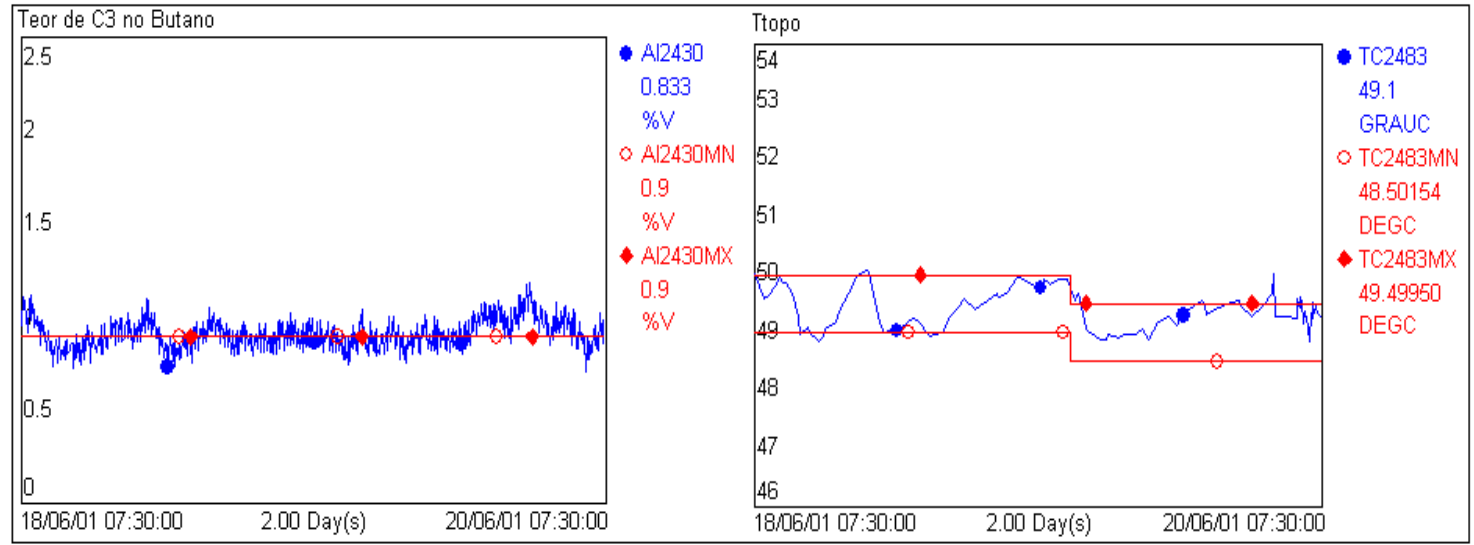

Figura 9.2.d1 - Variáveis controladas teste 3.

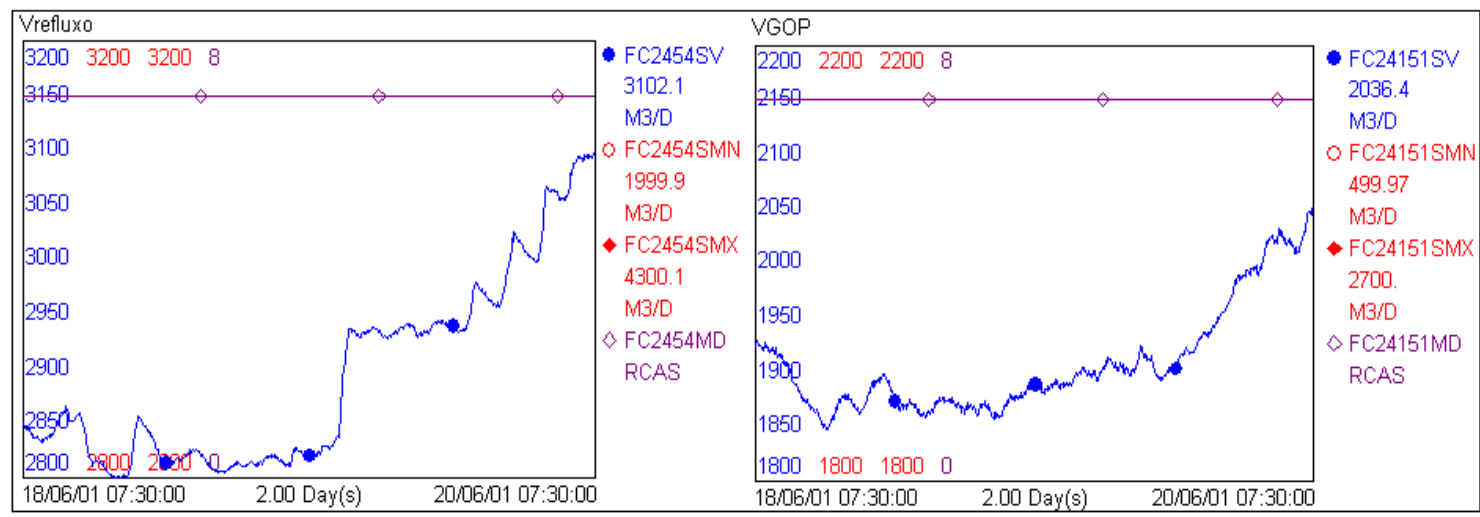

Figura 9.2.d2 - Variáveis manipuladas teste 3.

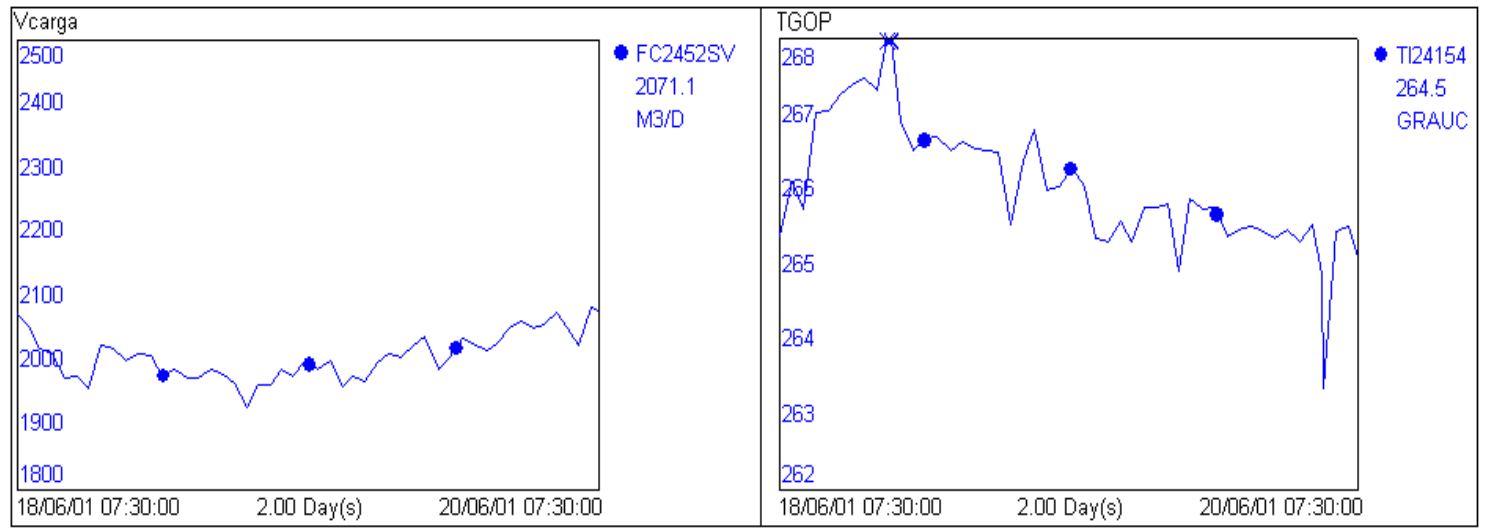

Figura 9.2.d3 - Variáveis perturbadoras teste 3. 


\subsection{1 - SICON - Sistema Integrado de Controle ( da Petrobras)}

Para que possamos implementar o controlador apresentado nos itens anteriores na planta industrial é necessário termos um conjunto integrado de softwares e computadores para execução dos algoritmos de forma segura. No caso da Petrobras este conjunto pode ser representado esquematicamente através da figura 9.2.e onde temos três interfaces descritas a seguir.

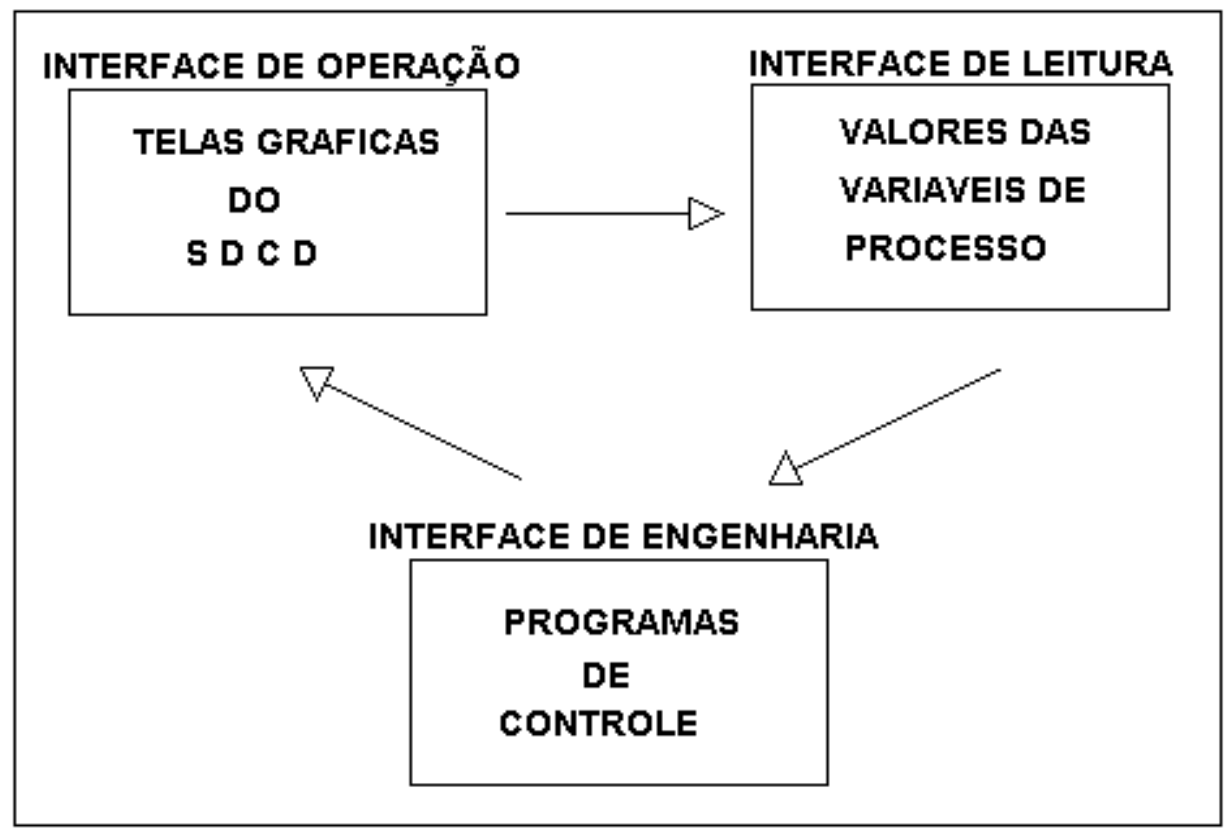

Figura 9.2.e - Interfaces de controle

1 - Interface de operação: esta interface é constituída pelas telas gráfica do SDCD (Sistema Digital de Controle Distribuído), onde os operadores atuam para colocar/retirar o programa de operação, alterar os limites de máximo/mínimo das variáveis quando for necessário, colocar/retirar variáveis do controle e monitorar o comportamento do programa e das variáveis.

2 - Interface leitura de dados: esta interface é constituída pelo programa PI (Plant Information $^{\mathrm{TM}}$ da Oil Systems) sendo a base de dados utilizada para leitura dos valores das variáveis utilizadas pelo programa de controle. 
3 - Interface de engenharia: esta interface é constituída de duas plataformas, sendo uma plataforma de engenharia e um computador de processo. A plataforma de engenharia é constituída basicamente por um computador PC com Windows, comunicando-se em rede com outros computadores. Nesta plataforma são executadas várias aplicações que permitem ao engenheiro de controle realizar as atividades de construção, sintonia, comissionamento e monitoramento das variáveis dos controladores através de uma interface gráfica adequada. O ambiente do Computador de Processo provê todos os serviços necessários à execução de aplicações de controle, como por exemplo, a aquisição de valores de processo do SDCD, validação destes valores para utilização pelo controlador, a execução dos algoritmos de controle, o envio das ações de controle de volta ao SDCD e a comunicação com a plataforma de engenharia. É importante notar que as aplicações de controle são executadas nesta plataforma e não dependem da plataforma de engenharia. A partir desta plataforma é feita a escrita dos valores de controle para o SDCD através da interface de comunicação Centap-plus ${ }^{\mathrm{TM}}$ da Yokogawa.

Nas figuras 9.2.f a 9.2.i, apresentamos as telas de configuração relativas à interface de engenharia, nestas telas temos os principais parâmetros de configuração e sintonia do controlador e das variáveis manipuladas, controladas e perturbadoras. Basicamente estas telas de configuração apresentam os seguintes campos de informação:

$\checkmark$ Descrição: apresenta uma descrição do controlador ou da variável correspondente.

$\checkmark$ Tags de entrada: são os valores lidos do SDCD ou fixados em um determinado valor.

$\checkmark$ Tags de saída: são valores que serão enviados para o SDCD ou PI.

$\checkmark$ Entradas: são parâmetros de configuração do controlador ou das variáveis que são preenchidos pelo engenheiro de controle.

$\checkmark$ Saídas: são informações fornecidas pelo programa a respeito da performance do controlador ou variável.

$\checkmark$ Faixa de validade: são os limites aceitáveis para as variáveis, fora destes limites a variável será desligada automaticamente pelo controlador.

$\checkmark$ Range da variável: normalmente são os limites mínimo e máximo de leitura das variáveis.

$\checkmark$ Auxiliares: são fatores de configuração adicionais que permitem a possibilidade de customização diferenciadas para cada controlador ou variável. 


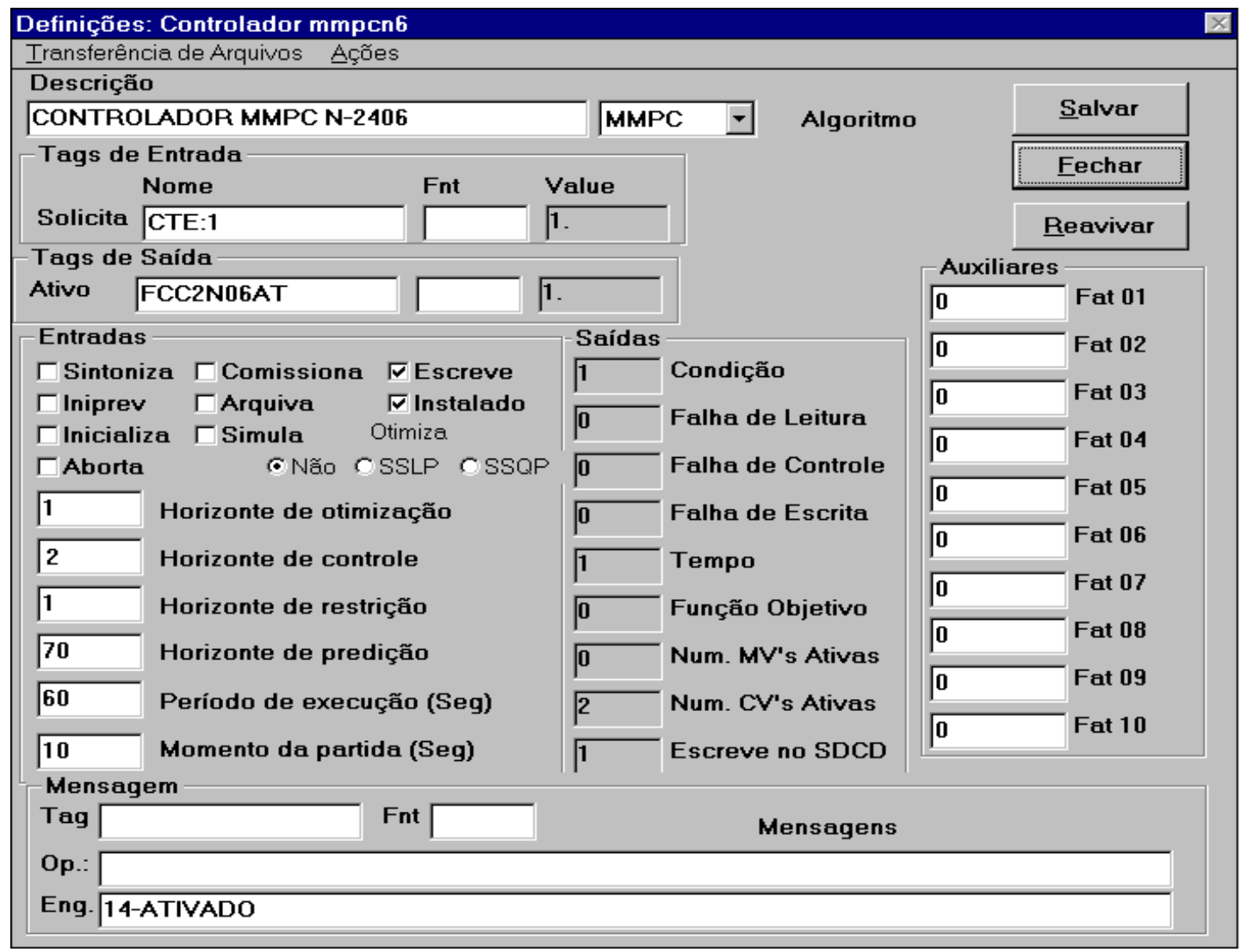

Figura 9.2.f - Tela de configuração/sintonia do controlador

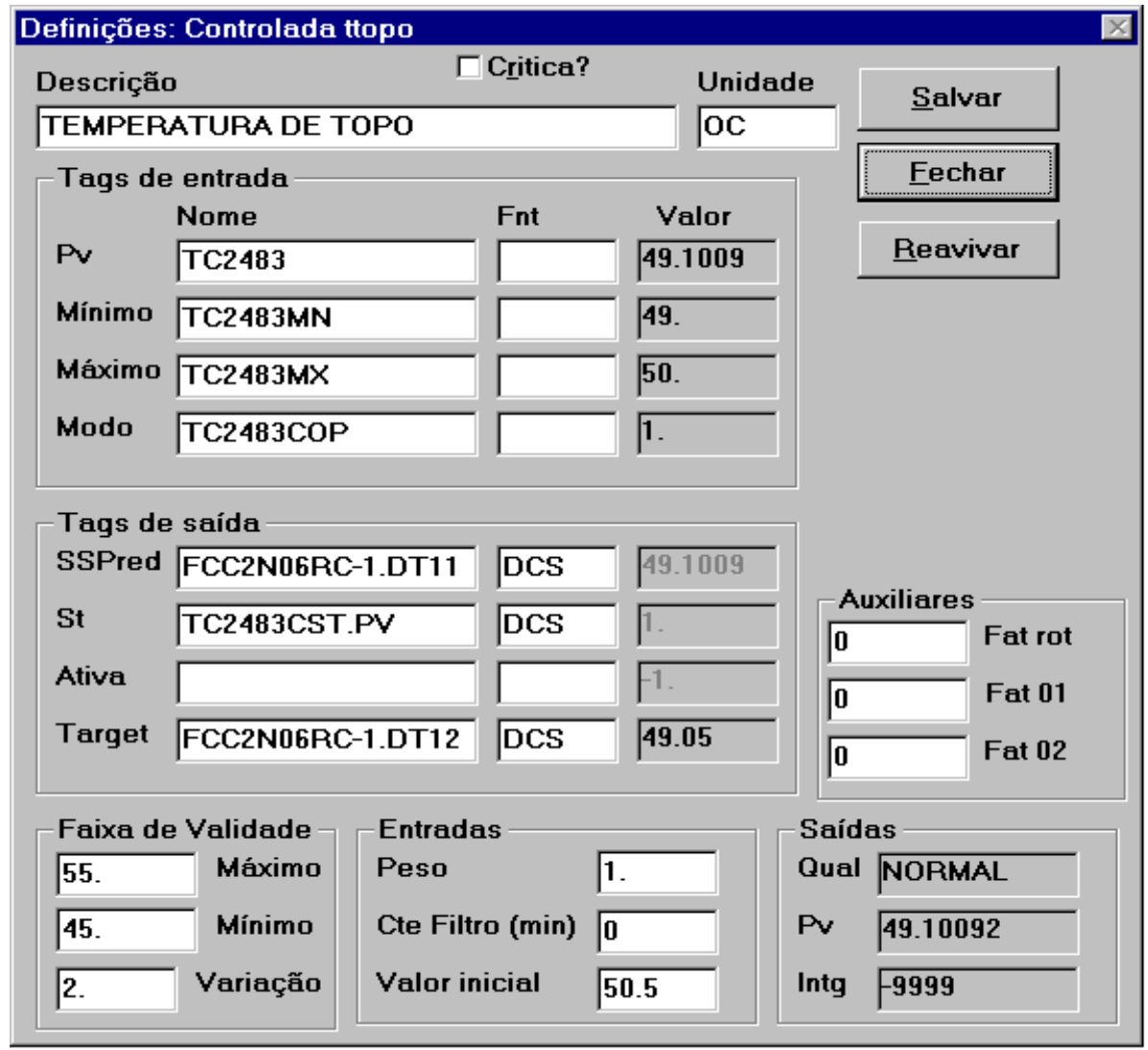

Figura 9.2.g - Tela de configuração/sintonia de variável controlada 


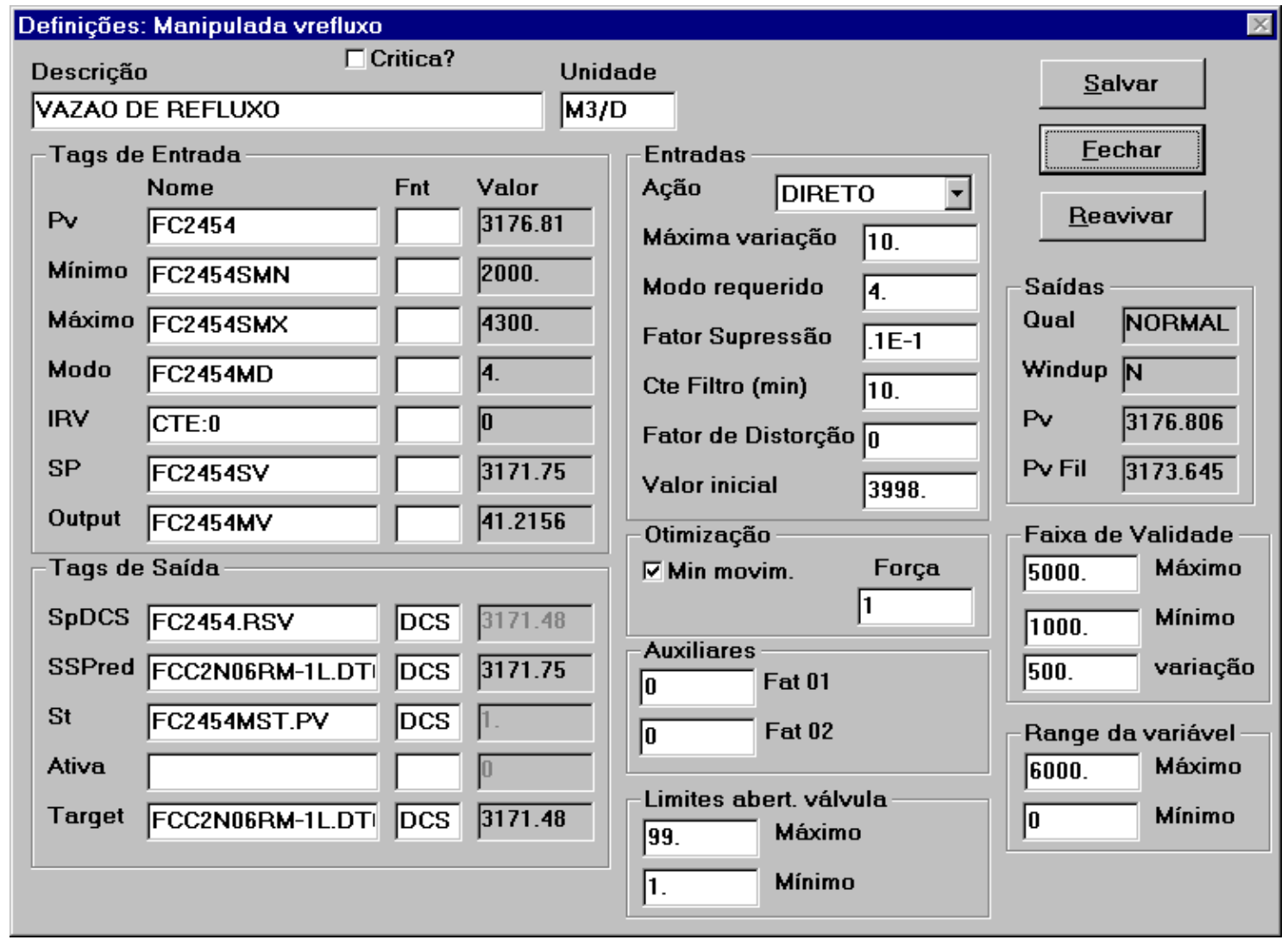

Figura 9.2.h - Tela de configuração/sintonia de variável manipulada

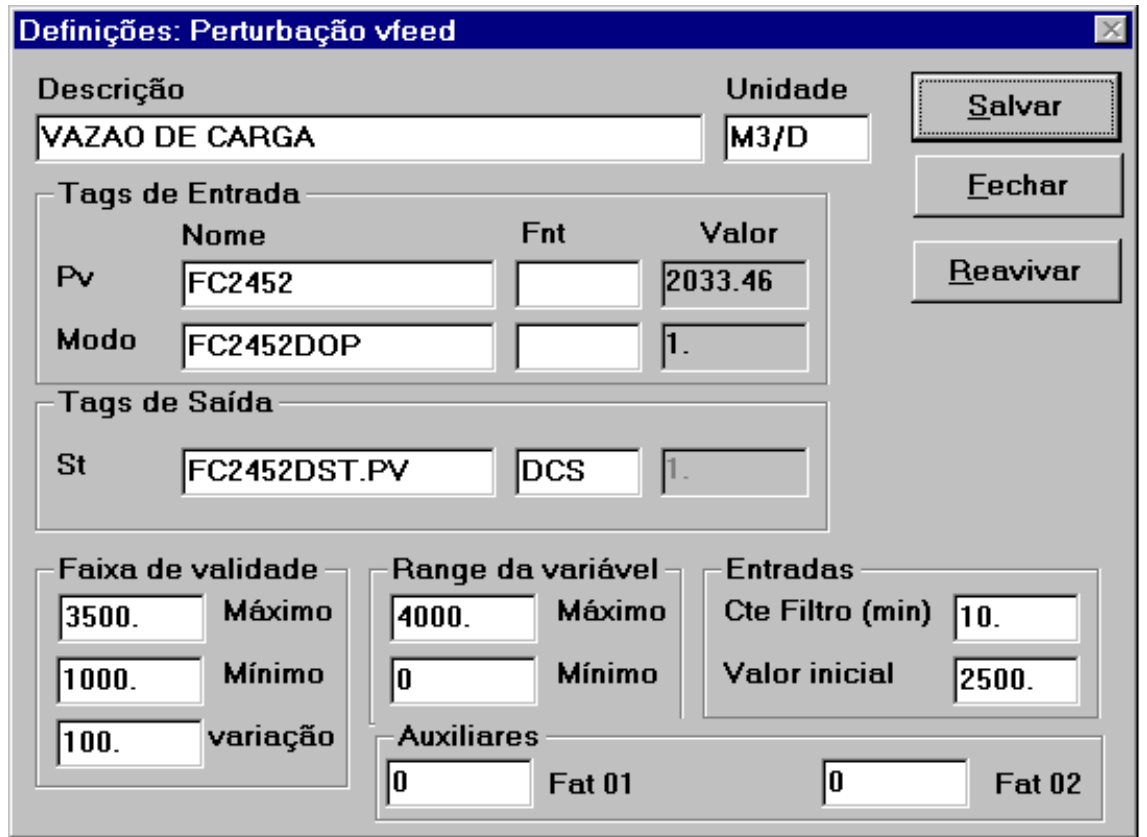

Figura 9.2.i - Tela de configuração de variável perturbadora 


\section{0}

\section{CONCLUSÕES E SUGESTÕES}

\section{1 - Conclus̃̃es}

Este trabalho é a primeira aplicação industrial de um controlador preditivo baseada no modelo de variáveis de estado montado a partir de resposta ao degrau analítica (ROSSMPC). As vantagens desse enfoque são:

$\checkmark$ Controlar sistemas com dinâmicas muito lentas, sem necessidade de memorizar vetores e matrizes de grandes dimensões.

$\checkmark$ Controlar sistemas com dinâmicas lentas e rápidas no mesmo controlador;

$\checkmark$ Prever as variáveis controladas em instantes de tempo diferentes para cada uma delas;

$\checkmark$ Controlar sistemas de grandes dimensões, ou seja, grande número de variáveis manipuladas e controladas. Isto possibilita controlar unidades de processo inteiras, sem grande esforço computacional.

$\checkmark$ Fazer adaptação dos ganhos e até mesmo dos parâmetros das dinâmicas sem grandes dificuldades.

Também nesse trabalho temos a descrição da primeira aplicação de um controlador baseado em múltiplos modelos onde o problema de otimização recai em um problema tipo min-max., ou seja, o controlador escolhe uma ação de controle que minimiza o erro para o pior modelo. Alguns autores argumentam que esse controlador seria demasiadamente conservativo no caso de modelos bastante diferentes. Este é o caso da aplicação aqui estudada onde os modelos têm características bastante diferentes. Por exemplo, o ganho do teor de $\boldsymbol{C}_{\mathbf{3}}{ }^{-}$em relação à vazão de refluxo para o modelo 1 é de 4.498E-4 e para o modelo 3 é de 1.679E-2, portanto 37 vezes maior.

Assim, esperava-se um comportamento não muito eficiente do controlador MMPC para a coluna depropanizadora. Entretanto, os resultados mostram que a performance, em termos de velocidade de resposta e tempo de estabilização, é perfeitamente compatível com as exigências práticas. Portanto, conseguiu-se um controlador com uma faixa de estabilidade bastante ampla e uma performance adequada. 
Este controlador exige um esforço na sintonia menor do que o QDMC e esta sintonia é mais adequada numa região de operação mais ampla do que a correspondente do QDMC. Embora o esforço computacional do MMPC seja maior que para o QDMC essa desvantagem não é importante para o caso estudado. No computador de controle de processo (Alpha 1000 server da Digital de $400 \mathrm{mhz}$ com 512 mb de memória ram) o tempo de computação do MMPC não chega a 1 segundo, portanto não significativo em comparação com o período de amostragem.

Tendo em vista estes resultados, espera-se que a utilização do MMPC se torne um padrão dentro da Petrobras.

Outro aspecto importante do trabalho foi a utilização do simulador de processos para levantamento do conjunto de modelos. Esta utilização se mostrou bastante interessante, pois através dela temos uma redução da necessidade de realização de testes na planta industrial, o que seria demorado e dispendioso. Além, de podermos pesquisar uma região mais ampla do processo, o que dificilmente seria conseguido na planta industrial.

\section{2 - Sugestões}

Em função de uma série de dificuldades e restrições do estudo, podemos sugerir alguns pontos para trabalhos futuros.

Os componentes do conjunto de modelos e o modelo nominal para predição foram escolhidos procurando abranger os diferentes pontos de operação do sistema e em função dos determinantes das matrizes de ganhos nestes pontos. Estes critérios são necessários, mas não suficientes, sendo que poderíamos incluir os critérios de estabilidade dos controladores na seleção dos modelos.

Neste estudo não incluímos a análise de estabilidade do sistema no projeto de controle. Sugerimos então que se inclua a análise de estabilidade no projeto de controle, até mesmo estudar algum algoritmo que otimize os parâmetros do controlador segundo os critérios de estabilidade. 
Também pode-se estudar alguma forma eficiente de incluir os critérios de estabilidade no cálculo da ação de controle. Isto poderia ser feito incluindo os critérios de estabilidade de Lyapunov no problema de controle.

No sentido de aumentar a estabilidade do controle, pode-se estabelecer algum critério de normalização das variáveis manipuladas, perturbações, controladas e modelos de forma a condicionar adequadamente o sistema de controle, desacoplando-o da melhor forma possível.

Em relação ao sistema estudado, o ideal seria que controlássemos efetivamente a contaminação de componentes pesados no topo da coluna através da medição do teor de $\mathrm{C}_{4}{ }^{+}$. Para isto seria necessário desenvolver uma inferência com qualidade superior ao da existente atualmente, que se baseia em correlação multilinear.

Outro aspecto que poderia ser desenvolvido seria a implementação de uma camada de otimização no controlador, isto permitiria um ganho em economia de energia, o que em uma coluna de fracionamento representa um grande incentivo, já que o processo de separação se baseia na introdução de energia para permitir a separação dos produtos. 


\section{REFERÊNCIAS BIBLIOGRÁFICAS}

ALMEIDA, E. 1999. Controle preditivo multimodelos de uma torre debutanizadora. 200p. Dissertação (Mestrado) - Escola Politécnica da Universidade de São Paulo.

ALMEIDA, E.; RODRIGUES, M. A.; ODLOAK D. (2000) Robust predictive control of a gasoline debutanizer column. Brazilian Journal of Chemical Engineering, v.17, no 04-07, p.967-977

ALMEIDA, E.; ZANIN, A. C.; ODLOAK, D. (1996). Advanced control and optimization of a crude vacuum unit. XI Congresso Brasileiro de Automática, - I Simpósio de Automática Aplicada, Anais p.23-28, São Paulo

ASTM-D86 (1970). Part 17, Petroleum Products-Fuels, Solvents, Burner Fuel Oils, Lubrificating Oils, Cutting Oils, Lubrificating Greases, Hydraulic Fluids. American Soc. For Testing \& Materials, Philadelphia, PA, p.8-20.

BOYD, S.; GHAOUI, L. E.; FERON, E.; BALAKRISHNAN, V. (1994). Linear Matrix Inequalities in System and Control Theory. SIAM, Philadelphia

CLARKE, D. W.; MOHTADI, C.; TUFFS, P.S. (1987). Generalized predictive controlPart I: the basic algorithm. Automatica, v.23, n.2, p.137-148

CLARKE, D. W.; MOHTADI, C.; TUFFS, P.S. (1987). Generalized predictive controlPart II: extensions and interpretations. Automatica, v.23, n.2, p.149-160

CUTLER, C. R.; RAMAKER, B. L. (1979). Dynamic matrix control - a computer control algoritm. AIChE $86^{\text {th }}$ National Meeting, Houton, TX

GARCIA, C. E.; MORARI, M. (1982). Internal model control - Part 1: A unifying review and some new results. Ind. Eng. Chem. Proc. Des. Dev., v.21, p.308-323

GARCIA, C. E.; MORARI, M. (1985). Internal model control - Part 2: Design procedure for multivariable systems. Ind. Eng. Chem. Proc. Des. Dev., v.24, p.472484

GARCIA, C. E.; MORARI, M. (1985). Internal model control - Part 3: Multivariable control law computation and tuning guidelines. Ind. Eng. Chem. Proc. Des. Dev., v.24, p.484-494

GARCIA, C. E.; MORSHEDI, A. M. (1986). Quadratic programing solution of dynamic matrix control (QDMC). Chem. Engng Commun., v.46, p.73-87 
GOUVÊA, M. T.; ODLOAK, D. (1997). ROSSMPC: A new way of representing and analysing the predictive controllers. Trans IchemE, v.75, Part A, p.693-708

HENSON, M. A. (1998). Nonlinear model predictive control: Current status and fucture directions. Computers Chem. Engng, v.23, n.2, p.187-202

HOYD, M.; LEE, J. H.; MORARI, M. (1993). Truncated step response model predictive control. Journal of Process Control, v.3, n.2, p.67-73

KOTHARE, K. V.; BALAKRISHNAN, V.; MORARI, M. (1996). Robust constrained model predictive control using linear matrix inequalities. Automatica, v.30, n.10, p.1361-1379

LEE, J. H.; MORARI, M.; GARCIA, C. E. (1994). State space interpretation of model predictive control, Automatica, v.30, n.4, p.707-717

LI, S.; LIM, K. Y.; FISHER, D. G. (1989). A state space formulation for model predictive control, AIChE Journal, v.35, n.2, p.241-249

LJUNG, L. (1987). System Identification: Theory for the user. Prentice-Hall, Englewood Cliffs, NJ

LUNDSTRÖM, P.; LEE, J. H.; MORARI, M.; SKOGESTAD, S. (1995). Limitations of dynamic matrix control. Computers Chem. Engng, v.19, n.4, p.409-421

MAGALHÃES, M. V. O.; ODLOAK, D. (1995). Multivariable quality control of a crude oil frationator. DYDORD'95, IFAC Conference, Dynanics and Control of Chemical Reactors, Distillation Columns and Batch Processes, p.583-488, Copenhagen

MIQUEL, J.; CASTELLS, F.(1993). Easy characterization of petroleum fractions, Hydroc. Processing, December 1993, p.101-105

MORARI, M.; DOYLE, J. (1986). A unifying frameworks for control system design under uncertainty and its implications for chemical process control. Proceedings of Chemical Process Control - CPC III, p.5-52

MORARI, M.; LEE, J. H. (1991). Model predictive control: The good, the bad, and the ugly. Proceedings of Chemical Process Control - CPC IV, San Padre Island, TX, p. $410-444$

MORARI, M.; LEE, J. H. (1999). Model predictive control: past, present and future. Computers Chem. Engng, v.23, p.667-682 
MORARI, M.; ZAFIRIOU, E. (1989). Robust process control. Prentice-Hall, Englewood Cliffs, NJ

MORO, L. F. L.; ODLOAK, D. (1994). Controle de conversores de craqueamento catalítico em leito fluido, I - Modelagem dinâmica. Boletim Técnico da Petrobras, v.37, n.1, p.3-12

MORO, L. F. L.; ODLOAK, D. (1994). Controle de conversores de craqueamento catalítico em leito fluido, II - Controle preditivo multivariável com restrições. Boletim Técnico da Petrobras, v.37, n.2, p.111-124

MORO, L. F. L.; ODLOAK, D. (1995). Constrained multivariable control of fluid cracking converters. Journal of Process Control, v.5, n.1, p.29-39

MORSHEDI, A. M.; CUTLER, C. R.; SKROVANEK, T. A. (1985). Optimal solution of dynamic matrix control with linear programming techniques (LDMC). Proc. Am. Control Conf., Boston, MA, p.199-208

NESTEROV, Y.; NEMIROVSKY, A. (1994). Interior-point polynomial methods in convex programming. SIAM, Philadelphia

ODLOAK, D. (1995). Stability analysis of the dynamic matrix controller, Braz. Jour. Chem. Engng, v.12, n.1, p.50-64

ODLOAK, D. (1996). A new state-space approach to model predictive control, Braz. Jour. Chem. Engng, v.13, n.3, p.152-167

PENG, D.Y.; ROBINSON, D.B. (1976). A new two constant equation of state. Ind. Eng. Chem. Fundamen., v.15, p.59-64

PRETT, D. M.; GILLETTE, R. D. (1979). Optimization and constrained multivarible control of catalytic cracking unit, AIChE $\mathbf{8 6}^{\text {th }}$ National Meeting, Paper 51-C, Houton, TX

PRETT, D. M.; GILLETTE, R. D. (1980). Optimization and constrained multivarible control of a catalytic cracking unit, Proc. Automatic Control Conf., San Francisco, CA, Paper WP5-C

RICHAlET, J.; RAULT, A.; TESTUD, J. L.; PAPON, J. (1978). Model predictive heuristic control: application to industrial processes. Automatica, v.14, n.5, p.413428

RODRIGUES, M. A.; ODLOAK, D. (2000). Output feedback MPC with guaranteed robust stability. Journal of Process Control, v.10, p. 557-572. 
ROUHANI, R.; MEHRA, R. K. (1982). Model algorithmic control (MAC): basic theoretical properties, Automatica, v.18, p.401

SCHITTKOWSKI, K. (1985/6). A Fortran Subroutine Solving Constrained NonLinear Programming Problems, Annals of Operations Research, vol. 5, p. 485-500

SPANDRI, R.; MORO, L. F. L.; ODLOAK, D. (1995). Constrained multivariable control of fluid catalytic cracking converters, a practical application. AIChE, Spring National Meeting, Paper 84b, Houston, TX

TRIERWEILER, J. O.; FARINA, L. A.; DURAISKI R. G. (2001). RPN Tuning strategy for model predictive control. DYCOPS, p.283-289, Jejudo Island, Korea

ZANIN, A. C.; ODLOAK, D. (1992). Advanced control of an atmospheric resid heater. Proceedings of the First International Instrumentation, Systems and Industrial Automation Meeting, p.33-42, Salvador, BA 


\section{APÊEDICE A \\ FUNÇÃO DE TRANSFERÊECIA DOS MODELOS IDENTIFICADOS}

Nas tabelas A1 a A6 apresentamos na forma de função de transferência os modelos identificados no capítulo 7. Os parâmetros da tabela obedecem seguinte equação:

$$
G(s)=\frac{b_{0}+b_{1} s}{s^{2}+a_{1} s+a_{2}} e^{-s \theta}
$$

\begin{tabular}{|c|c|c|c|c|c|c|c|}
\hline MODELO & $\mathrm{CV}$ & MV/DV & $b_{0}$ & $b_{1}$ & $a_{1}$ & $a_{2}$ & $\theta$ \\
\hline \multirow[t]{12}{*}{1} & \multirow[t]{4}{*}{$\mathrm{C}_{3}^{-}$} & $\mathrm{V}_{\mathrm{RF}}$ & $0.1094 \mathrm{e}-4$ & $0.4227 \mathrm{e}-4$ & 0.1090 & 0.0243 & 1 \\
\hline & & $\mathrm{V}_{\mathrm{GOP}}$ & $-0.3824 e-4$ & $-1.2055 \mathrm{e}-4$ & 0.1342 & 0.0111 & 1 \\
\hline & & $\mathrm{V}_{\text {carga }}$ & $0.5668 \mathrm{e}-2$ & $-0.0945 e-2$ & 0.3975 & 0.0850 & 1 \\
\hline & & $\mathrm{T}_{\mathrm{GOP}}$ & $-0.21740 \mathrm{e}-3$ & $-0.6856 e-3$ & 0.1342 & 0.0111 & 1 \\
\hline & \multirow[t]{4}{*}{$\mathrm{C}_{4}^{+}$} & $\mathrm{V}_{\mathrm{RF}}$ & $-0.2539 \mathrm{e}-3$ & $-0.2445 e-3$ & 0.1142 & 0.0086 & 1 \\
\hline & & $\mathrm{V}_{\mathrm{GOP}}$ & $0.6641 \mathrm{e}-3$ & $0.7159 \mathrm{e}-3$ & 0.1343 & 0.0087 & 1 \\
\hline & & $\mathrm{V}_{\text {carga }}$ & $-0.2482 e-3$ & $0.0617 \mathrm{e}-3$ & 0.1573 & 0.0141 & 1 \\
\hline & & $\mathrm{T}_{\mathrm{GOP}}$ & $0.0996 \mathrm{e}-1$ & $0.1074 \mathrm{e}-1$ & 0.1343 & 0.0087 & 1 \\
\hline & \multirow[t]{4}{*}{$\mathrm{T}_{\mathrm{TOPO}}$} & $\mathrm{V}_{\mathrm{RF}}$ & $-0.1116 e-3$ & $-0.0873 e-3$ & 0.1317 & 0.0073 & 1 \\
\hline & & $\mathrm{V}_{\mathrm{GOP}}$ & 0.0070 & 0.0013 & 2.2605 & 0.1366 & 1 \\
\hline & & $\mathrm{V}_{\text {carga }}$ & -0.0015 & -0.0004 & 1.9247 & 0.1353 & 1 \\
\hline & & $\mathrm{T}_{\mathrm{GOP}}$ & 0.1044 & 0.0194 & 2.2605 & 0.1366 & 1 \\
\hline
\end{tabular}

Tabela A1 - Parâmetros da função de transferência para o modelo 1 


\begin{tabular}{|c|c|c|c|c|c|c|c|}
\hline MODELO & $\mathrm{CV}$ & MV/DV & $b_{0}$ & $b_{1}$ & $a_{1}$ & $a_{2}$ & $\theta$ \\
\hline \multirow[t]{12}{*}{2} & \multirow[t]{4}{*}{$\mathrm{C}_{3}^{-}$} & $\mathrm{V}_{\mathrm{RF}}$ & $0.4220 \mathrm{e}-3$ & $-0.2722 \mathrm{e}-3$ & 1.6602 & 0.2525 & 1 \\
\hline & & $\mathrm{V}_{\mathrm{GOP}}$ & $-1.405 \mathrm{e}-4$ & $-2.1828 \mathrm{e}-4$ & 0.1322 & 0.0117 & 1 \\
\hline & & $\mathrm{V}_{\text {carga }}$ & $0.0521 \mathrm{e}-3$ & $0.1503 e-3$ & 0.2097 & 0.0279 & 1 \\
\hline & & $\mathrm{T}_{\mathrm{GOP}}$ & $-0.4740 \mathrm{e}-3$ & $-0.7365 e-3$ & 0.1322 & 0.0117 & 1 \\
\hline & \multirow[t]{4}{*}{$\mathrm{C}_{4}{ }^{+}$} & $\mathrm{V}_{\mathrm{RF}}$ & $-0.2891 \mathrm{e}-2$ & $-0.1572 \mathrm{e}-2$ & 0.7355 & 0.0561 & 1 \\
\hline & & $\mathrm{V}_{\mathrm{GOP}}$ & $0.0946 \mathrm{e}-2$ & $0.1605 \mathrm{e}-2$ & 0.1268 & 0.0123 & 1 \\
\hline & & $\mathrm{V}_{\text {carga }}$ & $-0.3885 \mathrm{e}-3$ & $-0.3830 \mathrm{e}-3$ & 0.1355 & 0.0148 & 1 \\
\hline & & $\mathrm{T}_{\mathrm{GOP}}$ & $0.1216 \mathrm{e}-1$ & $0.2064 \mathrm{e}-1$ & 0.1268 & 0.0123 & 1 \\
\hline & \multirow[t]{4}{*}{$\mathrm{T}_{\mathrm{TOPO}}$} & $\mathrm{V}_{\mathrm{RF}}$ & -0.0063 & -0.0034 & 2.0724 & 0.2428 & 1 \\
\hline & & $\mathrm{V}_{\mathrm{GOP}}$ & 0.0045 & 0.0002 & 0.8352 & 0.0812 & 1 \\
\hline & & $\mathrm{V}_{\text {carga }}$ & -0.0012 & -0.0012 & 0.8328 & 0.0693 & 1 \\
\hline & & $\mathrm{T}_{\mathrm{GOP}}$ & 0.0575 & 0.0020 & 0.8352 & 0.0812 & 1 \\
\hline
\end{tabular}

Tabela A2 - Parâmetros da função de transferência para o modelo 2

\begin{tabular}{|c|c|c|c|c|c|c|c|}
\hline MODELO & $\mathrm{CV}$ & MV/DV & $b_{0}$ & $b_{1}$ & $a_{1}$ & $a_{2}$ & $\theta$ \\
\hline \multirow[t]{12}{*}{3} & \multirow[t]{4}{*}{$\mathrm{C}_{3}^{-}$} & $\mathrm{V}_{\mathrm{RF}}$ & $0.1532 \mathrm{e}-2$ & $-0.0860 e-2$ & 1.1913 & 0.0912 & 1 \\
\hline & & $\mathrm{V}_{\mathrm{GOP}}$ & $-0.7811 \mathrm{e}-3$ & $-0.3770 e-3$ & 0.3402 & 0.0181 & 1 \\
\hline & & $\mathrm{V}_{\text {carga }}$ & $0.7698 \mathrm{e}-3$ & $0.8929 \mathrm{e}-3$ & 0.5633 & 0.0359 & 1 \\
\hline & & $\mathrm{T}_{\mathrm{GOP}}$ & $-0.7701 \mathrm{e}-2$ & $-0.3716 e-2$ & 0.3402 & 0.0181 & 1 \\
\hline & \multirow[t]{4}{*}{$\mathrm{C}_{4}{ }^{+}$} & $\mathrm{V}_{\mathrm{RF}}$ & $-0.1053 e-2$ & $-0.2637 e-2$ & 0.3524 & 0.0231 & 1 \\
\hline & & $\mathrm{V}_{\mathrm{GOP}}$ & $0.1335 \mathrm{e}-2$ & $0.0474 \mathrm{e}-2$ & 0.2471 & 0.0203 & 1 \\
\hline & & $\mathrm{V}_{\text {carga }}$ & $-0.3299 \mathrm{e}-3$ & $-0.2002 e-3$ & 0.2293 & 0.0190 & 1 \\
\hline & & $\mathrm{T}_{\mathrm{GOP}}$ & $0.1316 \mathrm{e}-1$ & $0.0467 \mathrm{e}-1$ & 0.2471 & 0.0203 & 1 \\
\hline & \multirow[t]{4}{*}{$\mathrm{T}_{\mathrm{TOPO}}$} & $\mathrm{V}_{\mathrm{RF}}$ & -0.0008 & -0.0034 & 0.4017 & 0.0365 & 1 \\
\hline & & $\mathrm{V}_{\mathrm{GOP}}$ & 0.0089 & 0.0064 & 1.8959 & 0.1946 & 1 \\
\hline & & $\mathrm{V}_{\text {carga }}$ & -0.0009 & -0.0012 & 0.8844 & 0.0799 & 1 \\
\hline & & $\mathrm{T}_{\mathrm{GOP}}$ & 0.0877 & 0.0634 & 1.8959 & 0.1946 & 1 \\
\hline
\end{tabular}

Tabela A3 - Parâmetros da função de transferência para o modelo 3 


\begin{tabular}{|c|c|c|c|c|c|c|c|}
\hline MODELO & $\mathrm{CV}$ & MV/DV & $b_{0}$ & $b_{1}$ & $a_{1}$ & $a_{2}$ & $\theta$ \\
\hline \multirow[t]{12}{*}{4} & \multirow[t]{4}{*}{$\mathrm{C}_{3}{ }^{-}$} & $\mathrm{V}_{\mathrm{RF}}$ & $0.4881 \mathrm{e}-3$ & $-0.1107 \mathrm{e}-3$ & 0.9881 & 0.0646 & 1 \\
\hline & & $\mathrm{V}_{\mathrm{GOP}}$ & $-0.1862 \mathrm{e}-3$ & $-0.1763 e-3$ & 0.2605 & 0.0091 & 1 \\
\hline & & $\mathrm{V}_{\text {carga }}$ & $0.2710 \mathrm{e}-3$ & $0.1854 \mathrm{e}-3$ & 0.5111 & 0.0265 & 1 \\
\hline & & $\mathrm{T}_{\mathrm{GOP}}$ & $-0.1781 \mathrm{e}-2$ & $-0.1686 \mathrm{e}-2$ & 0.2605 & 0.0091 & 1 \\
\hline & \multirow[t]{4}{*}{$\mathrm{C}_{4}{ }^{+}$} & $\mathrm{V}_{\mathrm{RF}}$ & $-0.0440 \mathrm{e}-2$ & $-0.1955 \mathrm{e}-2$ & 0.1510 & 0.0074 & 1 \\
\hline & & $\mathrm{V}_{\mathrm{GOP}}$ & $0.5084 \mathrm{e}-3$ & $-0.3732 e-3$ & 0.1041 & 0.0046 & 1 \\
\hline & & $\mathrm{V}_{\text {carga }}$ & $-0.1856 \mathrm{e}-3$ & $-0.3426 \mathrm{e}-3$ & 0.0967 & 0.0046 & 1 \\
\hline & & $\mathrm{T}_{\mathrm{GOP}}$ & $0.4862 \mathrm{e}-2$ & $-0.3570 \mathrm{e}-2$ & 0.1041 & 0.0046 & 1 \\
\hline & \multirow[t]{4}{*}{$\mathrm{T}_{\mathrm{TOPO}}$} & $\mathrm{V}_{\mathrm{RF}}$ & -0.0025 & -0.0039 & 0.8868 & 0.0840 & 1 \\
\hline & & $\mathrm{V}_{\mathrm{GOP}}$ & 0.0029 & 0.0055 & 0.8602 & 0.0392 & 1 \\
\hline & & $\mathrm{V}_{\text {carga }}$ & -0.0018 & -0.0004 & 1.8877 & 0.0669 & 1 \\
\hline & & $\mathrm{T}_{\mathrm{GOP}}$ & 0.0281 & 0.0527 & 0.8602 & 0.0392 & 1 \\
\hline
\end{tabular}

Tabela A4 - Parâmetros da função de transferência para o modelo 4

\begin{tabular}{|c|c|c|c|c|c|c|c|}
\hline MODELO & $\mathrm{CV}$ & MV/DV & $b_{0}$ & $b_{1}$ & $a_{1}$ & $a_{2}$ & $\theta$ \\
\hline \multirow[t]{12}{*}{5} & \multirow[t]{4}{*}{$\mathrm{C}_{3}^{-}$} & $\mathrm{V}_{\mathrm{RF}}$ & $0.5647 e-3$ & $-0.3536 e-3$ & 0.8165 & 0.0809 & 1 \\
\hline & & $\mathrm{V}_{\mathrm{GOP}}$ & $-0.4780 \mathrm{e}-3$ & $-0.1427 e-3$ & 0.3417 & 0.0259 & 1 \\
\hline & & $\mathrm{V}_{\text {carga }}$ & $0.6786 \mathrm{e}-3$ & $-0.1149 \mathrm{e}-3$ & 0.9003 & 0.0712 & 1 \\
\hline & & $\mathrm{T}_{\mathrm{GOP}}$ & $-0.6481 e-2$ & $-0.1935 e-2$ & 0.3417 & 0.0259 & 1 \\
\hline & \multirow[t]{4}{*}{$\mathrm{C}_{4}{ }^{+}$} & $\mathrm{V}_{\mathrm{RF}}$ & $-0.2862 \mathrm{e}-2$ & $-0.1206 e-2$ & 1.1691 & 0.0716 & 1 \\
\hline & & $\mathrm{V}_{\mathrm{GOP}}$ & $0.1352 \mathrm{e}-2$ & $0.1303 e-2$ & 0.3245 & 0.0180 & 1 \\
\hline & & $\mathrm{V}_{\text {carga }}$ & $-0.4581 \mathrm{e}-3$ & $0.2032 \mathrm{e}-3$ & 0.3565 & 0.0173 & 1 \\
\hline & & $\mathrm{T}_{\mathrm{GOP}}$ & $0.1833 \mathrm{e}-1$ & $0.1767 \mathrm{e}-1$ & 0.3245 & 0.0180 & 1 \\
\hline & \multirow[t]{4}{*}{$\mathrm{T}_{\mathrm{TOPO}}$} & $\mathrm{V}_{\mathrm{RF}}$ & -0.0021 & -0.0019 & 1.1676 & 0.1069 & 1 \\
\hline & & $\mathrm{V}_{\mathrm{GOP}}$ & 0.0081 & 0.0053 & 2.4190 & 0.1761 & 1 \\
\hline & & $\mathrm{V}_{\text {carga }}$ & $-0.7616 e-3$ & $-0.5323 e-3$ & 0.9818 & 0.0549 & 1 \\
\hline & & $\mathrm{T}_{\mathrm{GOP}}$ & 0.0766 & 0.0725 & 1.6731 & 0.1242 & 1 \\
\hline
\end{tabular}

Tabela A5 - Parâmetros da função de transferência para o modelo 5 


\begin{tabular}{|c|c|c|c|c|c|c|c|}
\hline MODELO & $\mathrm{CV}$ & MV/DV & $b_{0}$ & $b_{1}$ & $a_{1}$ & $a_{2}$ & $\theta$ \\
\hline \multirow[t]{12}{*}{ FD } & \multirow[t]{4}{*}{$\mathrm{C}_{3}^{-}$} & $\mathrm{V}_{\mathrm{RF}}$ & $0.5656 \mathrm{e}-3$ & $-0.2218 \mathrm{e}-3$ & 3.4948 & 0.5902 & 1 \\
\hline & & $\mathrm{V}_{\mathrm{GOP}}$ & -0.001452 & 0.00007413 & 2.6987 & 0.4023 & 1 \\
\hline & & $\mathrm{V}_{\text {carga }}$ & 0.0001125 & -0.0001138 & 0.4559 & 0.03227 & 1 \\
\hline & & $\mathrm{T}_{\mathrm{GOP}}$ & -0.01642 & -0.01324 & 1.438 & 0.184 & 1 \\
\hline & \multirow[t]{4}{*}{$\mathrm{C}_{4}{ }^{+}$} & $\mathrm{V}_{\mathrm{RF}}$ & - & - & - & - & 1 \\
\hline & & $\mathrm{V}_{\mathrm{GOP}}$ & - & - & - & - & 1 \\
\hline & & $\mathrm{V}_{\text {carga }}$ & - & - & - & - & 1 \\
\hline & & $\mathrm{T}_{\mathrm{GOP}}$ & - & - & - & - & 1 \\
\hline & \multirow[t]{4}{*}{$\mathrm{T}_{\mathrm{TOPO}}$} & $\mathrm{V}_{\mathrm{RF}}$ & -0.001235 & -0.001135 & 1.6380 & 0.09852 & 1 \\
\hline & & $\mathrm{V}_{\mathrm{GOP}}$ & 0.0020 & -0.0003 & 2.4298 & 0.0651 & 1 \\
\hline & & $\mathrm{V}_{\text {carga }}$ & -0.0003829 & 0.0001715 & 0.2295 & 0.02632 & 1 \\
\hline & & $\mathrm{T}_{\mathrm{GOP}}$ & 0.006970 & 0.001998 & 0.1731 & 0.01045 & 1 \\
\hline
\end{tabular}

Tabela A6 - Parâmetros da função de transferência para o modelo da planta industrial 